\title{
Detection of inorganic and metal nanoparticles using qNano - IZON Science's nanoparticle analysis system
}

By

Nadiah Ali

A thesis submitted to Victoria University of Wellington in fulfilment of the requirements for the degree of Master of Science in Physics

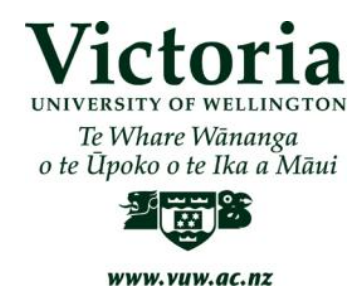

Victoria University of Wellington 2011 


\section{DECLARATION OF ORIGINALITY}

I, Nadiah Ali, declare that the Master by Research thesis entitled "Detection of inorganic and metal nanoparticles using qNano - IZON Science nanoparticle analysis system" is no more than 60,000 words in length including quotes and exclusive of tables, figures, appendices, bibliography, references and footnotes. This thesis contains no material that has been submitted previously, in whole or in part, for the award of any other academic degree or diploma. Except where otherwise indicated, this thesis is my own work.

Nadiah Nasreen Ali

$20^{\text {th }}$ January 2011 


\section{ACKNOWLEDGEMENT}

I would like to first thank the Almighty for giving me the knowledge, strength and drive to finish this Masters thesis.

A special thank you to my supervisor Associate Prof. Richard Tilley and Dr. Natalie Plank for all the support and guidance in completing this thesis. I honestly could not have done it without your assistance and understanding both academic and personal.

My deepest gratitude also goes out to all the staff member of IZON Science Ltd. especially Hans Van der Voorn, Rebecca Warr, Sam Yu, Murray Broom, Ben Glossop, and Anne Barnett for all the discussions regarding my work. Thank you also to Paul Atkins, Greg Bouwer, Linda Groenewegen, Sylvia and Megan for all the help they have given me at the start of my Masters degree.

To David Flynn who has patiently assisted me with imaging my samples, I thank you.

My appreciation also goes out to Richard Tilley's group members for all the conversations on my research. Your input is invaluable and I sincerely thank you.

Thank you also to the Head of Department Prof. John Spencer, the Science Faculty especially Ms. Shona de Sain, and Ms Suzie Paima for making my Masters manageable especially when I was going through a difficult time.

Thank you also to my parents, Cairo M., Leah Rowbotham, Katherine Beijeman, Kavitha Gopal Krishnan, Amanda Creiglow, Wai Ruu Siah, Phillip Luey, and Ayla Middleton for being an awesome support crew.

My final thank you to Foundation of Research Science and Technology (FoRST) for granting me the TIF funding. 


\section{ABSTRACT}

This thesis investigates the potential and capability of the qNano instrument, a nanoparticle analysis research instrument developed by IZON Science Ltd. The qNano operates on the basis of the Scanning Ion Occlusion Spectroscopy (SIOS) technology which is similar to the Coulter technique also known as resistive pulse sensing. This coupled with an adjustable nanopore and IZON's recently developed Variable Pressure Module (VPM) makes qNano a versatile nanoparticle analysis apparatus.

In this study the potential of the instrument to detect inorganic and metal nanoparticles is explored. Polystyrene, silica, gold, silver, and magnetic nanoparticles have been used in this research to better understand the system and its components and to discover the capacity of the instrument to detect these particles. Using polystyrene nanoparticles, it was found that the adjustable nanopore exhibit membrane softening and follows the Mullins effect. Each of the apertures also possesses a characteristic window of operation, which refers to a range of stretch setting for accurate particle detection. The adjustable nanopore also allows for sizing particles with different diameters. The effect of surface functionality in the signal output was also investigated.

Gold nanoparticles were synthesized via the citrate reduction method and the study of gold nanoparticles suspended in IZON's standard buffer solution was looked into in depth. It was found that the gold nanoparticles aggregate in the buffer solution due to the presence of potassium chloride $(\mathrm{KCl})$ salt which screens the electrostatic stabilization provided by the citrate ions. Silver nanoparticles show similar aggregation. With the concentration of gold and $\mathrm{KCl}$ remaining constant, resistive pulses caused by gold aggregates were detected after a period of three hours. Varying the concentration of gold nanoparticles shows that larger aggregates are formed as opposed to small aggregates with higher population. Identification of different types of particles present in a solution is yet to be achieved at this stage. 
Silica and magnetic nanoparticles were studied briefly. Successful measurements of magnetic particles were achieved with the aid of the pressure module. However, aggregates of magnetic particles were also formed and can be seen with the naked eye after solution was left standing overnight. Casein stabilized silica particles shows erratic current readings as the ethanol used in fabricating the silica particles collapse the casein micelle structure thus making the stabilization by casein void.

Overall, this thesis has shown the qNano instrument's ability in detecting nanoparticles other than biological molecules. This thesis also shows the potential of qNano as a versatile research instrument. The prospect of further research using this instrument is tremendous especially in characterising in greater detail inorganic and metal nanoparticles. 


\section{CONTENT}

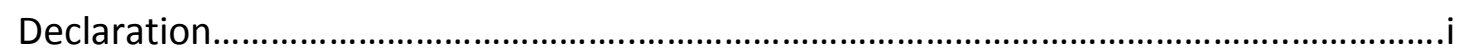

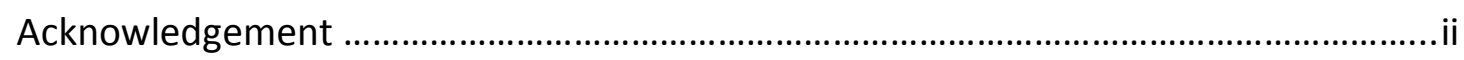

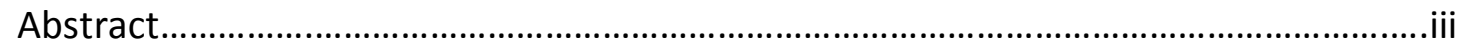

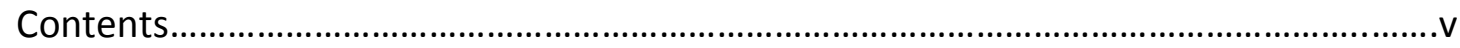

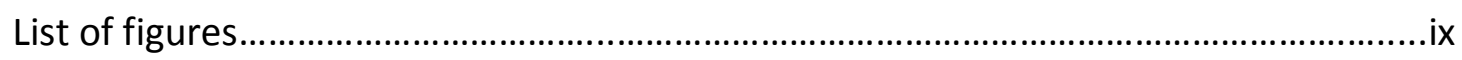

List of Tables.....................................................................................................................

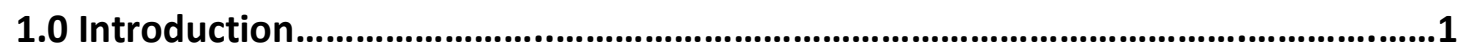

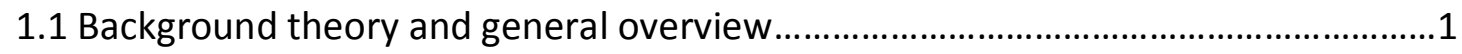

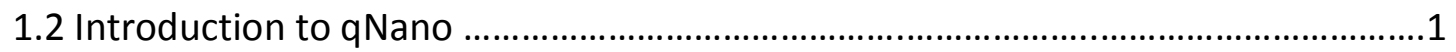

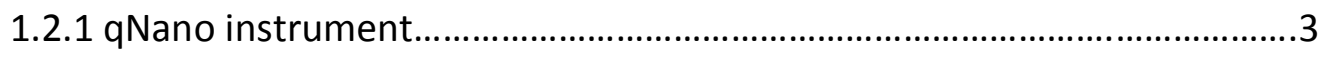

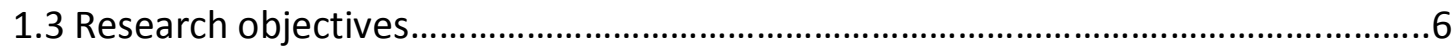

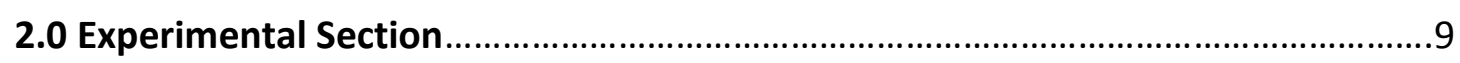

2.1 qNano software - Izon Control suite ………...........................................................

2.2 Thermoplastic polyurethane (TPU) apertures......................................................21

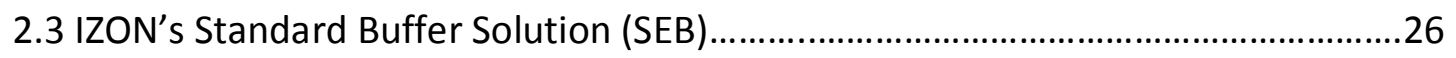

2.4 Variable Pressure Module (VPM) - A current development..................................27

3.0 Measurements of polystyrene particles.................................................................29

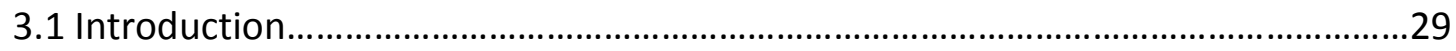

3.1.1 Setup of the qNano measurement system............................................29

3.2 Measurements on qNano with submicron particles...............................................30

3.2.1 Aperture calibration ..................................................................................

3.2.1.1 Aperture stability and current profile........................................31

3.2.1.1.1 Experimental methods..............................................31

3.2.1.1.2 Results and Analysis.....................................................32

3.2.1.1.3 Discussion .....................................................................33

3.2.1.2 Optimising stretch for a single particle set...............................34 
3.2.1.2.1 Experimental methods 35

3.2.1.2.2 Results and Analysis..................................................36

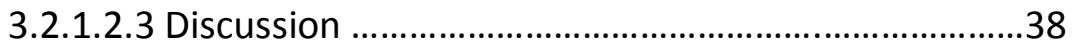

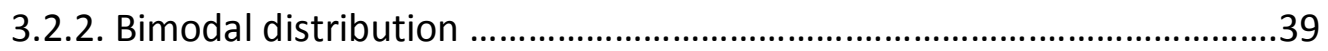

3.2.2.1 Experimental methods.................................................................40

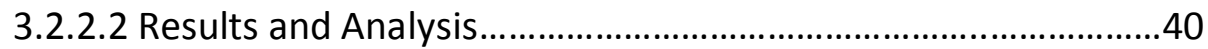

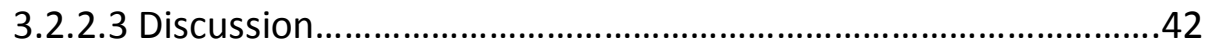

3.2.3 Effect of surface functionality of polystyrene particles.........................43

3.2.3.1 Synthesis of polystyrene and carboxylated polystyrene

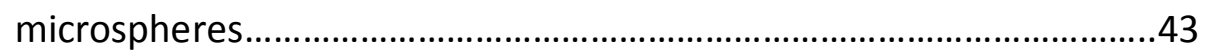

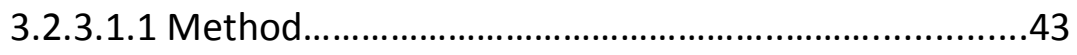

3.2.3.1.2 Risk assessment..........................................................4

3.2.3.1.3 Results and Analysis......................................................45

3.2.3.1.4 Synthesis discussion....................................................50

3.2.3.1.5 Synthesis conclusion..................................................52

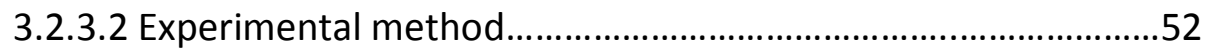

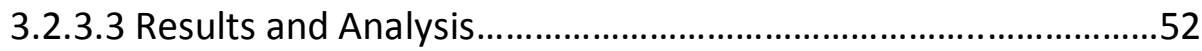

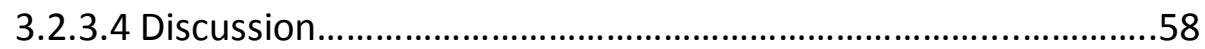

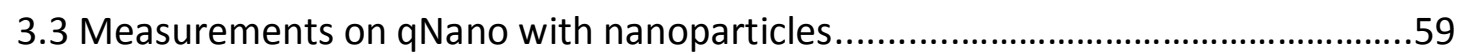

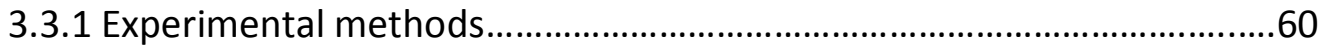

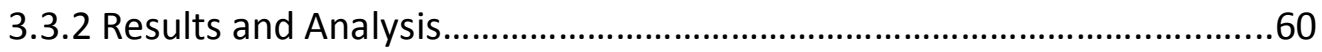

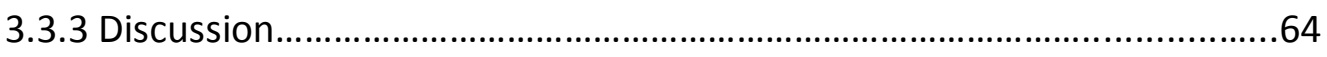

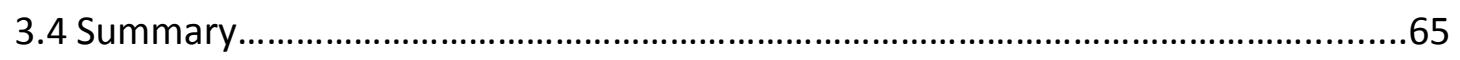

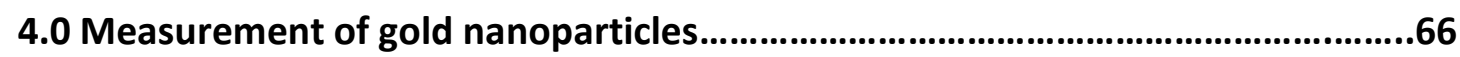

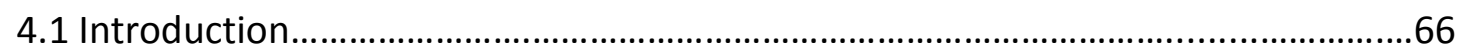

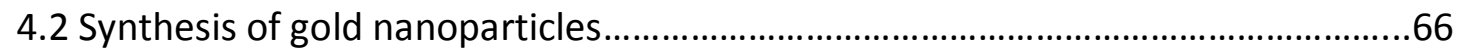

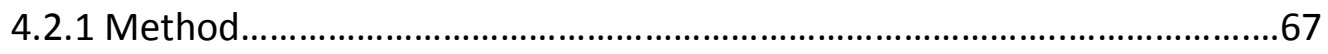

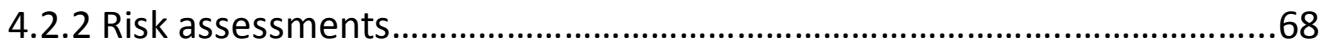

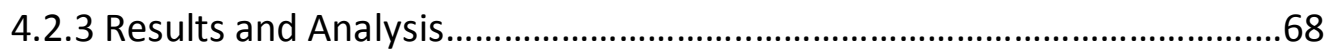

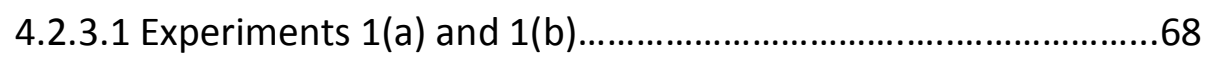

4.2.3.2 Experiment 2(a) and 2(b) .....................................................72 


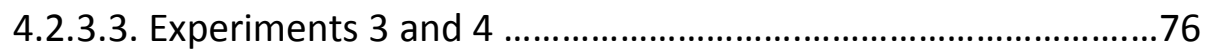

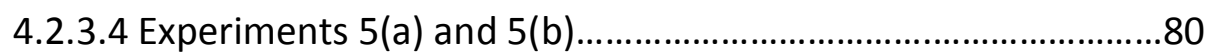

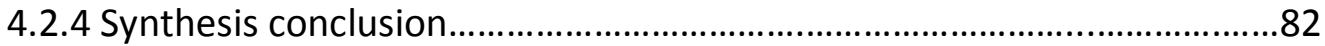

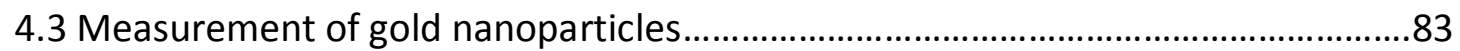

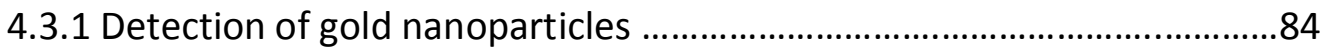

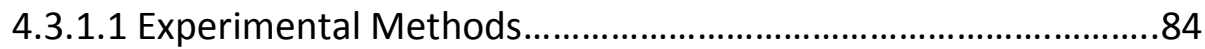

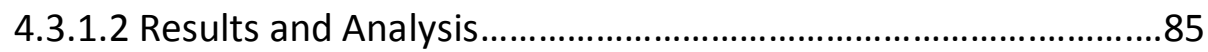

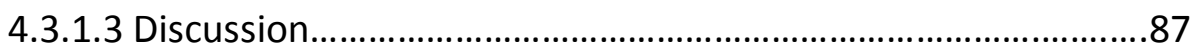

4.3.2 Induced aggregation of gold nanoparticles..............................................89

4.3.2.1 Experimental Methods.................................................................89

4.3.2.2 Results and Analysis...................................................................90

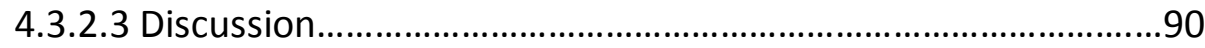

4.3.3 Aggregation time of gold nanoparticles ................................................91

4.3.3.1 Experimental Methods...............................................................91

4.3.3.2 Results and Analysis................................................................92

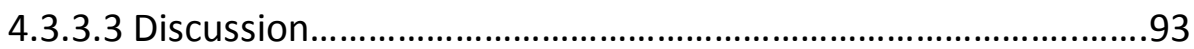

4.3.4 Detection of gold nanoparticles with a bandwidth of $50 \mathrm{kHz}$................93

4.3.4.1 Experimental Methods..................................................................94

4.3.4.2 Results and Analysis.................................................................94

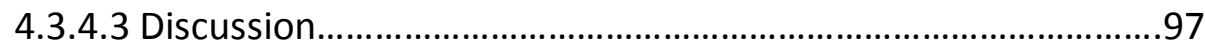

4.3.5 Effect of concentration of gold nanoparticles .......................................98

4.3.5.1 Experimental Methods...............................................................99

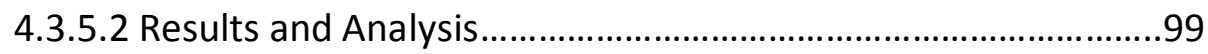

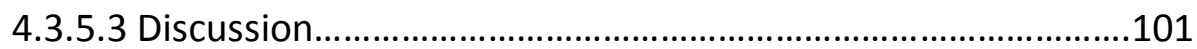

4.3.6 Identification of two species of nanoparticles mixture: gold and

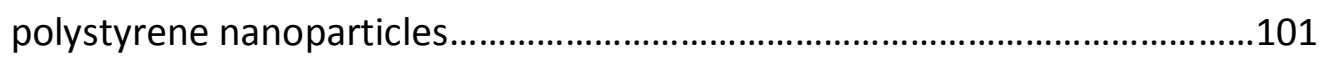

4.3.6.1 Experimental Methods.............................................................102

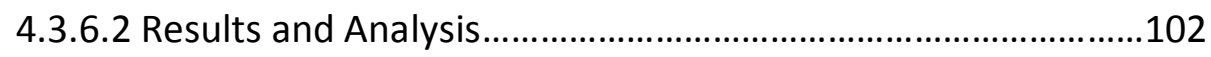

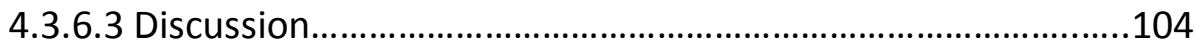

4.4 Measurement of gold nanoparticles under Variable Pressure Module

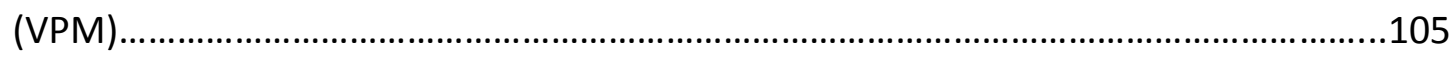

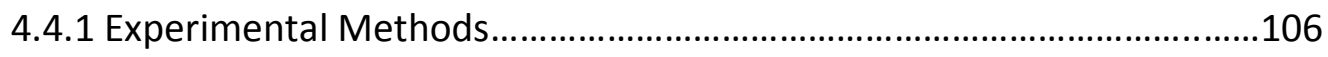




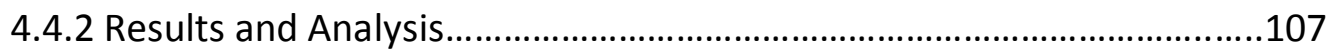

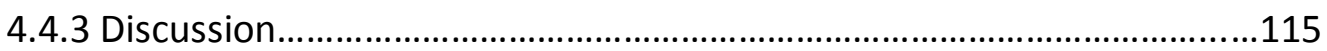

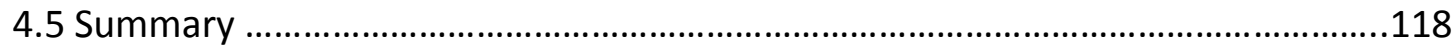

5.0 Measurements of other inorganic and noble metals nanoparticles ................119

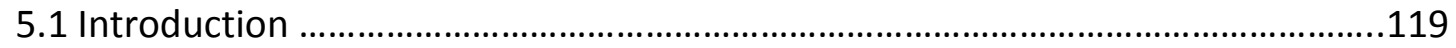

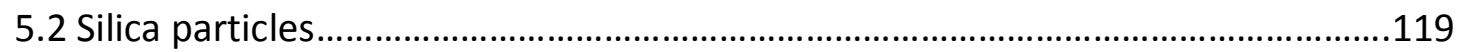

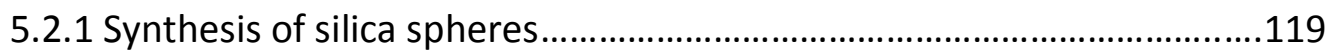

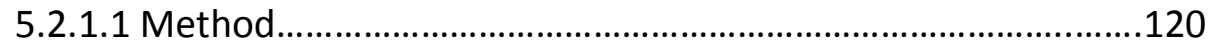

5.2.1.2 Risk Assessment .................................................................121

5.2.1.3 Results and Analysis.............................................................121

5.2.1.4 Synthesis discussion .................................................................125

5.2.1.5 Synthesis conclusion...............................................................126

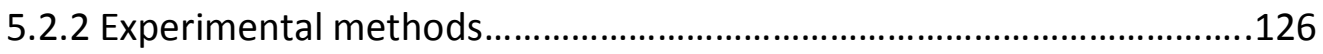

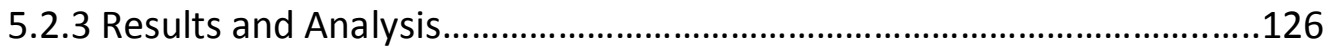

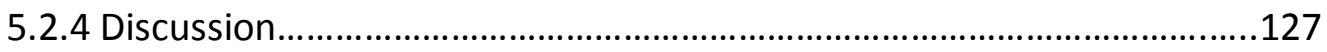

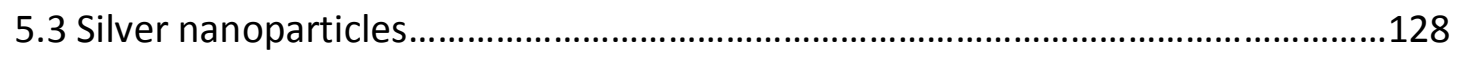

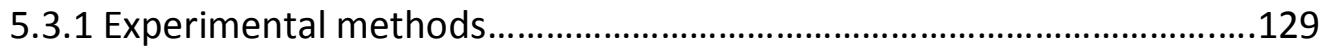

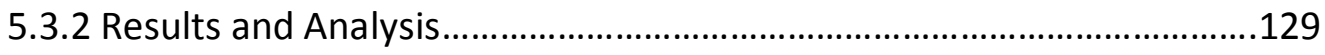

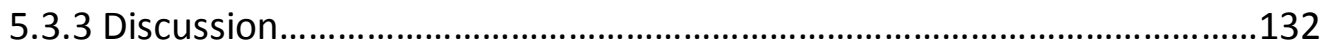

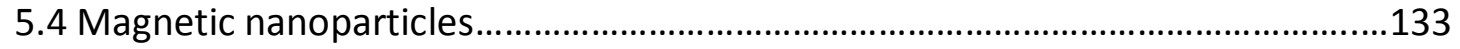

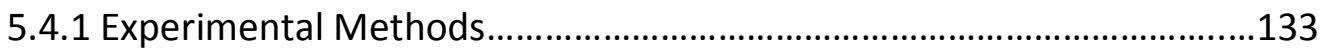

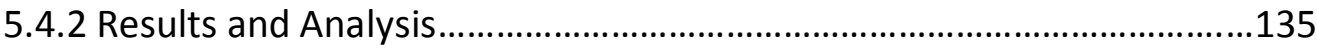

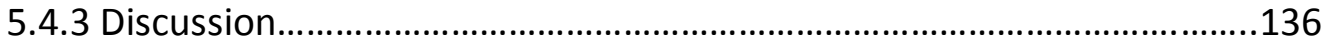

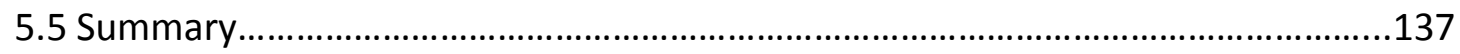

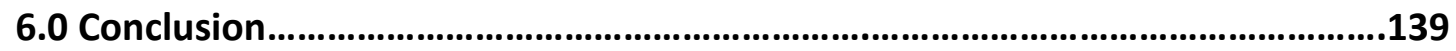

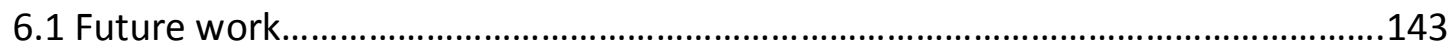

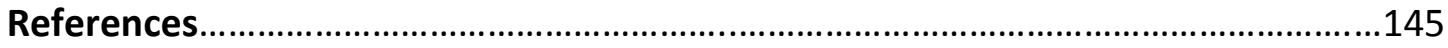




\section{LIST OF FIGURES}

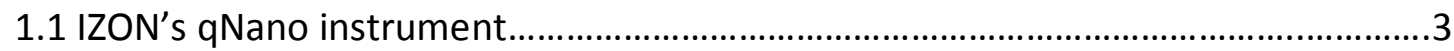

1.2 Schematic diagram of the fluid cell (not to scale) ....................................................

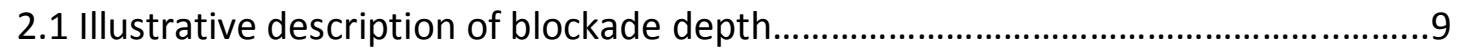

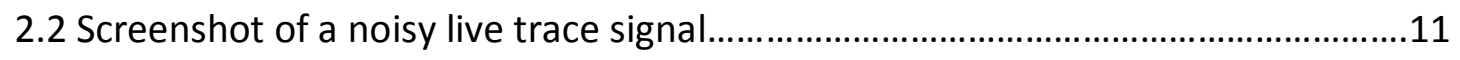

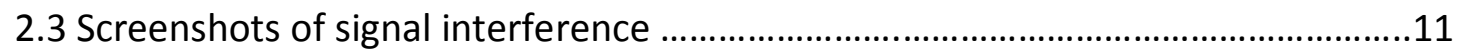

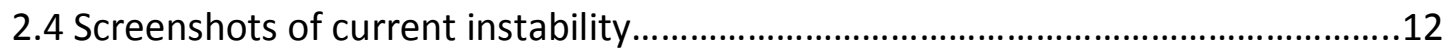

2.5 The default user interface screen...........................................................................

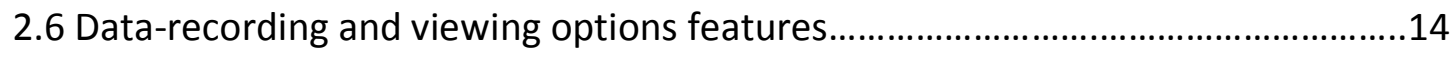

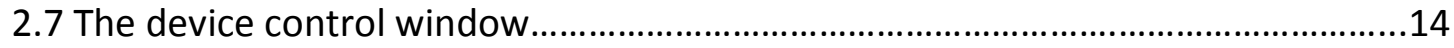

2.8 The main window to observe the data captured by qNano...................................15

2.9 A typical current signal that runs through the aperture. .........................................15

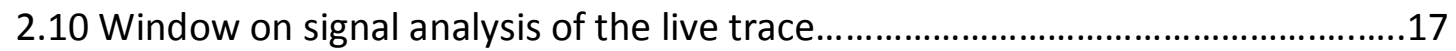

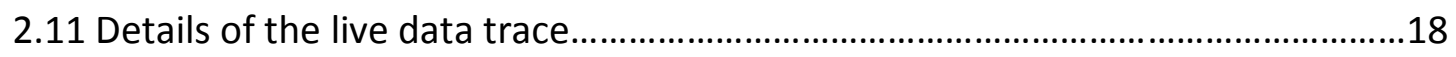

2.12 Data file for recorded measurements.................................................................18

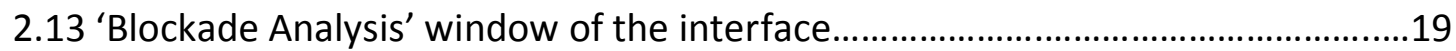

2.14 Signals window comparison between raw signals and normalised

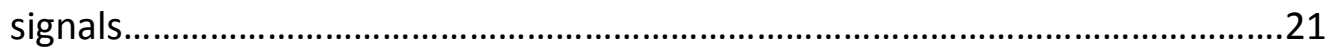

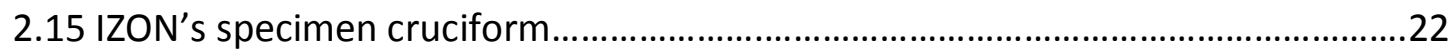

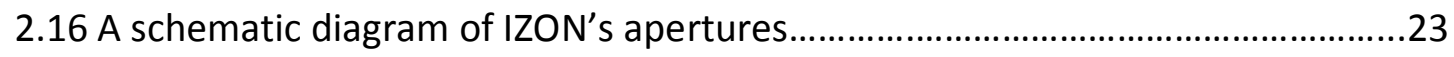

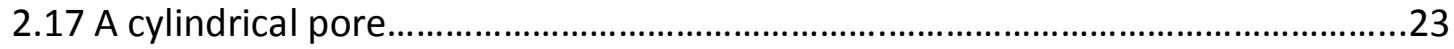

2.18 Expected signals from blockades with (a) A conical nanopore and (b) a

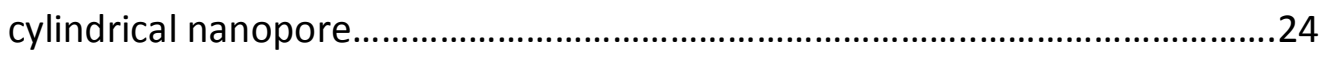

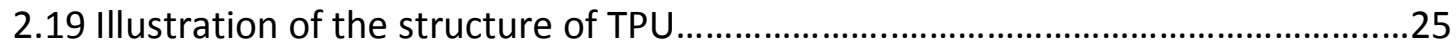

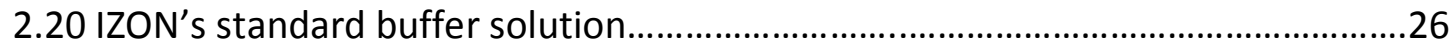

2.21 An image of the variable pressure module (VPM) which

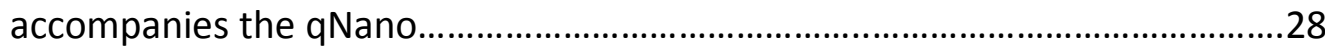

3.1 Example of the carboxylated particles with sizes $220 \mathrm{~nm}$ and $780 \mathrm{~nm}$..................30

3.2 A typical current profile of apertures manufactured by IZON.................................32 
3.3 Screenshot of an example of an unstable current running. .33

3.4 Blockade counts detected as a function of pore stretch. .36

3.5 Blockade counts of $780 \mathrm{~nm}$ particles through the aperture at four different pore stretches.

3.6 Noise spikes generated from hand movement over the fluid cell as seen in the 'Normalised Signal' window..... 38

3.7 A plot of bimodal distribution .41

3.8 SEM images of polystyrene samples PS01 and PS02 .46

3.9 Size distribution for PS01 as analysed using SemAfore. .47

3.10 SEM images of polystyrene samples PSO3 and PSO4 48

3.11 Size distribution of polystyrene particles PSO4. 48

3.12 SEM image of the synthesized carboxylated polystyrene particles samples at $\mathrm{x} 10,000$ magnifications

3.13 Size distribution of carboxylated polystyrene particles .50

3.14 Distribution of CTPS01 and PSO2 as they translocate through the nanopore .53

3.15 Distribution of PSO4 and 800nm IZON particles as they translocate through the nanopore.

3.16 Histogram plot showing the distribution of blockade magnitudes for PSO2 and CTPS01 particles suspended in SEB.

3.17 Histogram showing the distribution of blockade magnitudes for PSO4 and IZON's 800nm polystyrene particles suspended in SEB

3.18 Histogram showing the distribution of blockade duration for both PSO2 and CTPS01 particles suspended in SEB. .57

3.19 Histogram showing the distribution of blockade duration for both PSO4 and IZON's 800nm polystyrene particles suspended in SEB.

3.20 Scatter plot of particle blockades for both $100 \mathrm{~nm}$ and $70 \mathrm{~nm}$ polystyrene particles suspended in SEB

3.21 Screenshots for $100 \mathrm{~nm}$ blockades.

3.22 Screenshots for $70 \mathrm{~nm}$ blockades.

3.23 Histogram for blockade magnitude of $100 \mathrm{~nm}$ and $70 \mathrm{~nm}$ particles

3.24 Histogram for blockade duration of $100 \mathrm{~nm}$ and $70 \mathrm{~nm}$ particles. 64 
4.1 Images for $1(\mathrm{a})$ and $1(\mathrm{~b})$ gold nanoparticles and its respective

EDS mapping

4.2 Size distribution for samples of experiment 1 (a)....................................................

4.3 Absorption peak for gold nanoparticles form experiment 1(a)............................71

4.4 Images for 2(a) and 2(b) gold nanoparticles at 50, 000x magnifications...............73

4.5 EDS mapping of a selected spot of sample from experiment 2(a).......................74

4.6 UV-Vis absorbance spectrum on both nanoparticles from experiments 2(a)

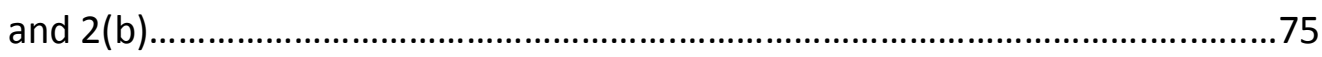

4.7 TEM images for gold nanoparticles from experiment 3 and experiment 4 ..........77

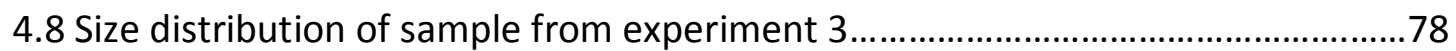

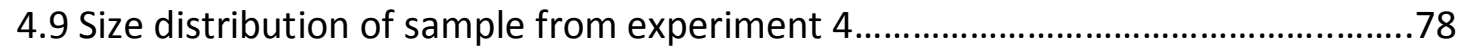

4.10 (a) UV-Vis absorbance spectrum on nanoparticles from experiment 3 .............79

4.10 (b) UV-Vis absorbance spectrum on nanoparticles from experiment 4 ...............79

4.11 TEM images for experiment 5(a) and 5(b) gold nanoparticles.............................81

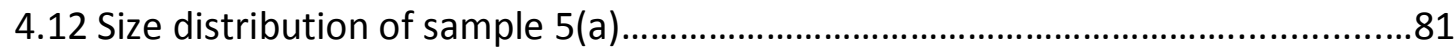

4.13 Screenshot of blockade event detected for gold nanoparticles..............................85

4.14 Distribution of gold nanoparticles translocating through the nanopore..............86

4.15 Histogram of blockades magnitude for gold nanoparticles detected...................87

4.16 Distribution of 70nm IZON's polystyrene nanoparticles and $11 \mathrm{~nm}$ gold nanoparticles suspended in $\mathrm{Na}$-Cit buffer

4.17 Screenshot of $100 \mathrm{~nm}$ polystyrene blockades .95

4.18 Screenshot of the signal with standard buffer solution and $11 \mathrm{~nm}$ gold

Nanoparticles.

4.19 Distribution of both IZON's $100 \mathrm{~nm}$ polystyrene nanoparticles and $11 \mathrm{~nm}$

gold nanoparticles in IZON's standard buffer solution . .96

4.20 Screenshot of the normalised signal with standard buffer solution and

$11 \mathrm{~nm}$ gold nanoparticles.

4.21 Scatter plot of gold nanoparticles dispersed in the standard buffer solution

at different concentration.

4.22 A histogram of blockade magnitudes of gold nanoparticles dispersed in standard buffer solution at different concentration. 100

4.23 Distribution of blockade magnitudes of 70nm polystyrene nanoparticles, 
100nm gold nanoparticle and a mixture of polystyrene and gold nanoparticles

4.24 Distribution of the time taken for both the polystyrene and gold nanoparticles to go through the nanopore .104

4.25 Image of $100 \mathrm{~nm}$ particles purchased from BB International. 106

4.26 Screenshot of $100 \mathrm{~nm}$ polystyrene nanoparticles going through the nanopore. .108

4.27 Distribution of selected 100nm polystyrene nanoparticles suspended in IZON's buffer 108

4.28 Particle blockades as a function of duration. 109

4.29 Histogram of blockade duration for polystyrene nanoparticles and gold nanoparticles at different concentration.

4.30 Blockade counts as particle dilution gets less concentrated.

4.31 Blockade events of gold nanoparticles in a 1:100 and 1:10 dilution suspended in IZON's buffer solution

4.32 Blockade events of $100 \mathrm{~nm}$ gold nanoparticles at different dilution. .114

4.33 Plots of blockade events for gold in a dilution of 1:100, 1:50, and 1:10.....

5.1 Images of silica particles at $\times 20,000$ magnification

5.2 Images of silica particles at $\times 20,000$ magnification

5.3 Size distribution of silica particles (Experiment 1)

5.4 Images of silica particles at $\times 20,000$ magnification

5.5 Size distribution of silica particles (Experiment 4)

5.6 Distribution of silica particles suspended in IZON's standard buffer solution.....127

5.7 Blockade events of $100 \mathrm{~nm}$ polystyrene (PS) nanoparticles and silver nanoparticles suspended in the standard buffer solution.

5.8 Distribution of blockade magnitudes of $60 \mathrm{~nm}$ silver nanoparticles and $100 \mathrm{~nm}$ polystyrene nanoparticles suspended in the standard buffer solution....

5.9 Distribution of blockade duration for $60 \mathrm{~nm}$ silver and $100 \mathrm{~nm}$ polystyrene (PS) nanoparticles suspended in the standard buffer solution.

5.10 Distribution of magnetic nanoparticles at a different dilution suspended in IZON's standard electrolyte buffer.

5.11 The distribution of magnetic nanoparticles at different concentration 
suspended in IZON's standard electrolyte buffer...

.136

5.12 Image of magnetic nanoparticles suspended in IZON's standard buffer solution. 


\section{LIST OF TABLES}

3.1 Data for blockades obtained for each set of particles and the mix . .40

3.2 Reaction condition used to synthesise polystyrene spheres. .44

3.3 Risk Evaluation. .45

4.1 Reaction conditions in synthesizing gold nanoparticles by citrate reduction method. 67

4.2 Risk evaluation. .68

4.3 Summary of experiments for the measurement of gold nanoparticles .84

4.4 Running conditions for polystyrene and gold nanoparticles using VPM. 107

5.1 Reaction conditions for the synthesis of silica particles. 120

5.2 Risk evaluation. 121 


\subsection{INTRODUCTION}

\subsection{Background theory and general overview}

At present, particle sizing is mostly done via microscopy analysis and light scattering technology. Techniques such as ultracentrifugation, chromatography as well as gel electrophoresis have also been employed [3]. Predominantly, the transmission electron microscope (TEM) and scanning electron microscope (SEM) are used to take images of particle samples and analysis is done by taking a set of samples and manually sizing the particles. This method is widely acceptable and gives an almost accurate size representation of the entire specimen's population.

The other key method used for particle sizing is the dynamic light scattering (DLS) method, sometimes referred to as the Photon Correlation Spectroscopy (PCS) or Quasi-Elastic Light Scattering (QELS). This method utilises a laser beaming through the sample that calculates the velocity of particles' Brownian motion. Particles suspended in a solution will scatter the laser beam and the diameter of a particle is determined by the time it takes for the reflected laser to be detected. Due to the Brownian motion of particles in the sample, the intensity of the laser beam fluctuates which ultimately gives only an average value of the particle diameter. Sizing with this technique also limits the ability to distinguish different sized particles that are present in the same sample. This is where qNano - a technology introduced by IZON- proves to be a better tool for particle size analysis.

\subsection{Introduction to qNano}

IZON's qNano coupled with the dynamically resizable nanopore is a comprehensive research instrument that opens up numerous opportunities with regards to nanoparticle analysis [6]. qNano offers researchers a host of other useful options as follows: the ability to view particle interaction in real-time, the detection of single particles suspended in a buffer solution, a resizable nanopore that allows size selection and separation, and many others. Initially, qNano is available to researchers for particle sizing - which is the focus of this thesis. Over time, qNano has now been 
developed as a tool for concentration analysis as well as real-time chemical reaction and particle interaction.

The qNano technology utilises the change in current flow to give information about individual particles suspended in a buffer solution. IZON call this technology the Scanning Ion Occlusion Spectroscopy (SIOS). The SIOS technology is based on the Coulter technique also known as resistive pulse sensing. The Coulter technique is a method that has been predominantly used by researches to measure the size and concentration of biological molecules (viruses and bacteria) and other particles that are suspended in an electrolyte solution [1-5, 8,10, 13-15,17,28]. This technique usually consists of two reservoirs that holds the particles' solution and is separated by a membrane that has a nanopore that creates a pathway between the two reservoirs. The solution usually contains an ionic carrier that flows under a voltage bias, creating an electric current in the system. A block in the nanopore creates a resistance in the electrical current and this resistance is the key information in analysing the particles or molecules under investigation. Analysing the resistance data provides information on the size, structure, concentration, surface charge, and particle mobility $[2,6-8,14,17]$.

In addition to the SIOS technology, IZON's qNano also uses an adjustable nanopore made from thermoplastic polyurethane (TPU). The adjustable nanopore allows researchers to tune the nanopore to a desired pore stretch. This is done by mechanically stretching the aperture that is fixed on the qNano instrument evenly along the $x$ and $y$-axis, which gives control over the size of the nanopore.

Its key selling point is the claims that this technology is the "world's most comprehensive nanoparticles analysis system in a single instrument" [88]. The straightforwardness and easy to use instrument is one of the main advantages of qNano. Due to the novelty of this technology, some issues have been discovered while this research was conducted. These include susceptibility of the instrument to external electrical disturbance (due to its high sensitivity) and issues regarding the dependability of the TPU aperture. This thesis aims at addressing both of these issues in later chapters. 
IZON has collaborations with many research institutes worldwide on various research areas such as biomedicine, non-biological nanoparticle research, apertures or nanopore characterisation, virology, as well as DNA sequencing and molecular analysis $[6,11,34,86,88,89]$. All these researches are of high interest especially in DNA sequencing as this is currently one of the hottest topics studied in relation to nanopore research. Details of discussion of this thesis will be given in section 1.3 but in summary, this thesis will examine the use of qNano in sizing particles in the sub-micron $(0.1 \mu \mathrm{m}$ $1 \mu \mathrm{m}$ ) and nano (less than $100 \mathrm{~nm}$ ) region. The two types of particles used in this research are mainly polystyrene and gold nanoparticles. This thesis will also discuss the capability of qNano, the characteristics of the TPU aperture, and the interaction of particles with the standard buffer produced by IZON.

\subsection{1 qNano instrument}

IZON's qNano is a comprehensive research tool that allows extensive nanoparticle analysis. The instrument itself is designed to be lightweight, portable, and the operation of the instrument is very straightforward. Figure 1.1 is an image of the qNano instrument.

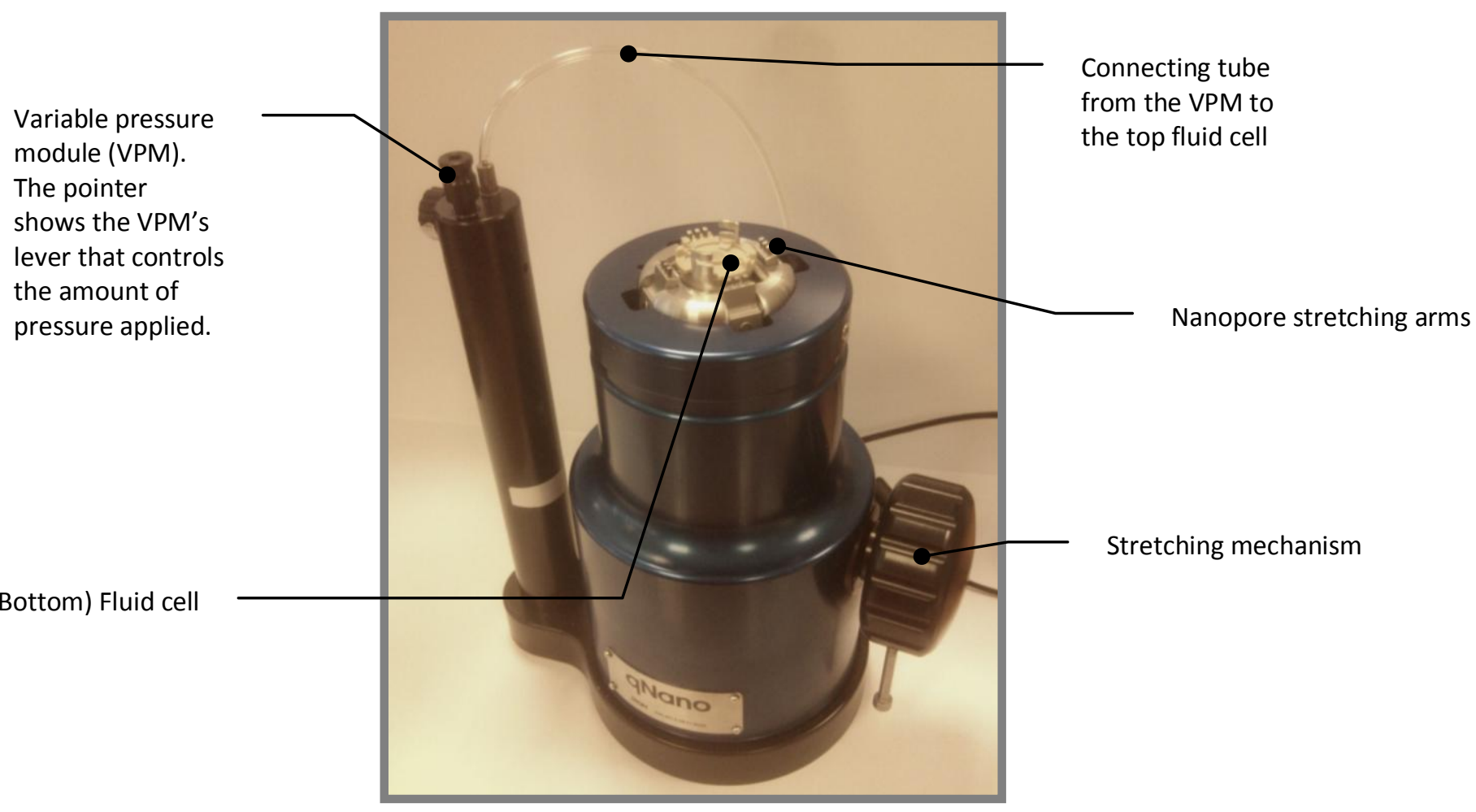

Figure 1.1: IZON's qNano instrument with the attached variable pressure module (VPM). Figure shows the main parts of the instrument that will be used by the user. 
The variable pressure module (VPM) is a manometer that gives a pressure of $0-15 \mathrm{~cm}$ of water $(0-1.5 \mathrm{kPa})$ to the fluid cell. This pressure is applied by adjusting the manometer lever, which transfers pressure via the connecting tube that attaches to the top of the fluid cell. The nanopore is sandwiched between the top and bottom fluid cell and is held by the stretching arms at four ends. These stretching arms, which sit on an $\mathrm{X}$ - and $\mathrm{Y}$-axis, are controlled by the stretching mechanism that allows the nanopore to be mechanically tuned. This feature allows users to control the size of the nanopore suitable for the needs of the measurements.

The qNano's Scanning Ion Occlusion Spectroscopy (SIOS) technology uses electrophoresis to drive particles that are suspended in the solution from one chamber to another. The SIOS technology measures the resistance of the electrical current as particles go through the nanopore. This resistance is the basis of the operation of qNano as it gives information about the particles, which include size and structure. The system has an aperture that is wedged between an upper and a lower fluid cell. The schematic diagram of the fluid cell is given in figure 1.2.

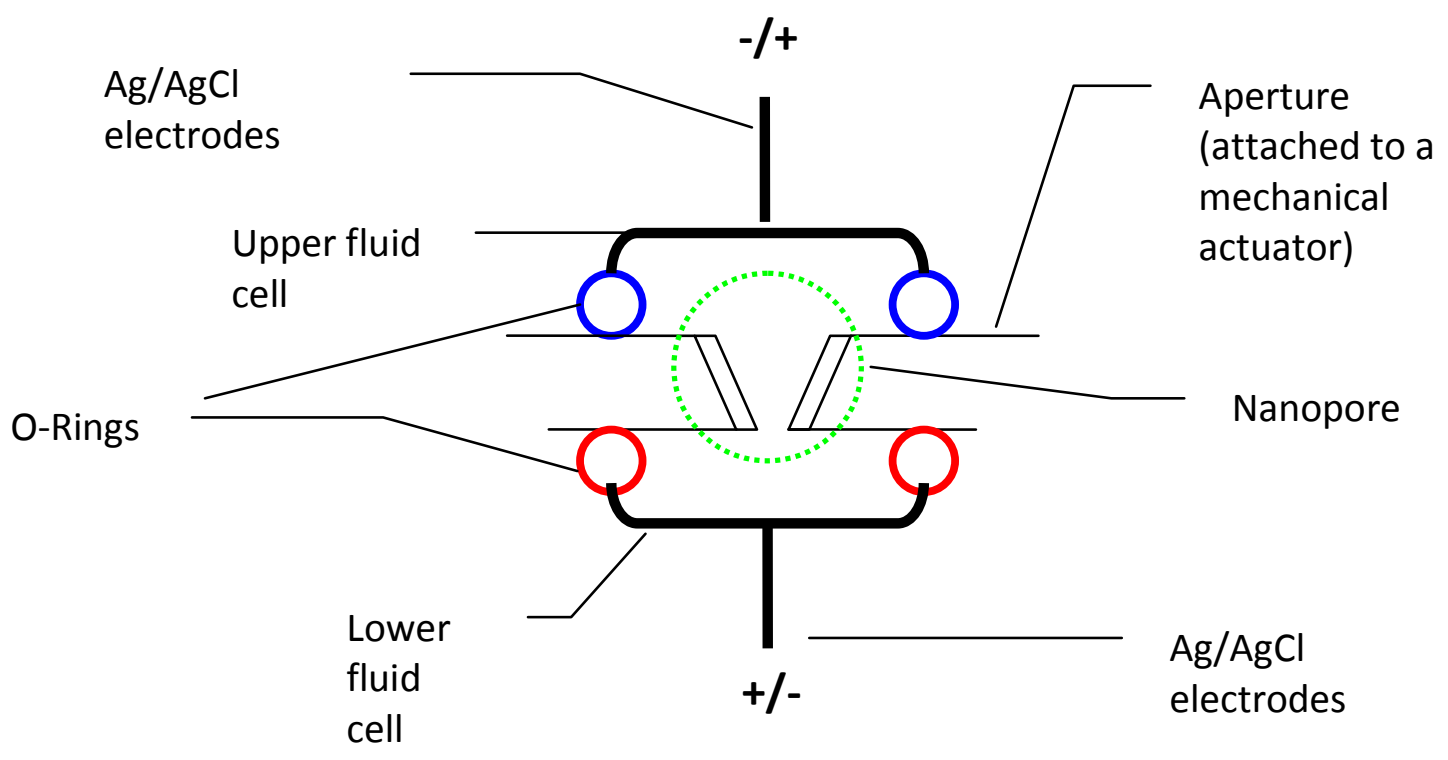

Figure 1.2: Schematic diagram (not to scale) of the fluid cell's main component. The diagram shows how the fluid cell system is assembled where the aperture is sandwiched between the top and bottom fluid cell. 
Figure 1.2 details the main components in the fluid cell. The electric potential applied to the system will cause the ionic carrier in the buffer solution to flow. This flow of charged particles through the nanopore creates a small current and this current is detected by qNano. The current caused by the flow of ions are in the nano Amps (nA) region. Any particles that upset or obstruct the flow of ions through the nanopore will decrease the ionic flow. This dip in ionic flow is termed 'blockades' for this thesis. The blockades detected can give information about the particles' shape (measured by the signal pattern and the duration of blockade), and diameter (measured by the depth of blockade) $[5,6,88,89]$.

There are three types of mechanism that transfers the particles from one fluid cell to the other: diffusion, electrophoresis, and electro-osmotic [5]. These combined processes can be described by the Poisson-Nernst-Plank theory $[16,28,86]$ which states that the current density $(J)$ due to the moving charged particles with electric field that goes through a membrane is given by equation 1 .

$$
J=-D\left(\frac{\partial C}{\partial z}\right)-\left(\frac{\zeta F}{R T}\right) D C\left(\frac{\partial \varphi}{\partial z}\right) \pm C v
$$

$J$ is the current density (only when considering transport of ions), D is diffusion coefficient, $\mathrm{C}$ is concentration of the sample, $\mathrm{z}$ is the cylindrical polar coordinate which is parallel to the normal of the surface of the aperture with the origin through the centre of the pore opening on the trans side, $\zeta$ is the charge on the particle, $F$ is the Faraday constant, $R$ is the gas constant, T is the temperature, $\varphi$ is the electric potential, and $v$ is the electro-osmotic velocity.

The first term $-D\left(\frac{\partial C}{\partial z}\right)$ on the right hand side of equation 1 is the diffusive transport term - derived from the gradient of electrochemical potential of the electrolyte. The second term $-\left(\frac{\zeta F}{R T}\right) D C\left(\frac{\partial \varphi}{\partial z}\right)$ is the electrophoretic term, and the last term $\pm C v$ is the electro-osmotic term. The diffusion term is assumed negligible, as the concentration of the buffer solution in the fluid cell is constant. The electro-osmotic 
term can typically be neglected for tuneable pores therefore leaving the particle flux to be only due to the electrophoretic term [34].

It is assumed that electrophoresis is the main driving force in moving the particles from one fluid chamber to another. The electrophoretic mobility $(\mu)$ is defined as the speed of the particles' per electric field gradient as given in equation 2 [89]

$$
\mu=\frac{\left(\frac{L}{\Delta t}\right)}{\left(\frac{E}{L}\right)}=\frac{L^{\wedge} 2}{E \Delta t}
$$

$\mathrm{L}=$ length,$\Delta \mathrm{t}=$ translocation duration, $\mathrm{E}=$ Electric field

Equation (2) applies for a long, thin cylindrical model of the pore. The equation shows that the translocation duration is related to the electrophoretic mobility $(\mu)$ of the particles.

\subsection{Research objectives}

At present, studies on IZON's tuneable nanopore are actively ongoing to understand how the aperture behaves as well as interpreting the information it provides. One of the first reported works on IZON's tuneable nanopore are by Sowerby et al. [6] which shows that it is possible to fabricate a resizable aperture by penetrating an elastomeric membrane with a coned-shaped needle probe. The aperture is resizable due to its elastomeric properties that allow elastic reduction that is centrally focused within the nanopore to overcome the macroscopic defect. The success at making a tuneable nanopore opens up a wide arrange of research possibilities which include gating of molecules as well as serving as an advanced tool for nanoparticle analysis.

The work of Willmott et al. [86] focuses on characterising the aperture's response to reversible stretching. Willmott et al. [86] found that within a window of stretch range, the current is proportional to the aperture stretch to the power of $n . n$ has a value of 
between 0.9-2.3 depending on the aperture used. Below the aperture's lower stretch limit, no effect is observed to the current flow when the pore is further stretched. One explanation offered to this observation is that the current is only influenced by the material directly next to the nanopore, which has been stretched to its limit during aperture fabrication.

Another work of Willmott et al. [34] focuses on using the resizable aperture to study the rate of particle translocation as a function of concentration and electrophoretic mobility of particles in aqueous solution. The study found that the rate of carboxylated polystyrene particles is proportional to the pressure applied and the concentration of the particles in the solution. Silica particles that have been coated with carboxylic acid and amine were used for the study of electrophoretic mobility and it was found that the mobility varies as a function of $\mathrm{pH}$. WIIlmott's research also shows that the nanopore has a "sensing zone" which is important in the understanding of how the qNano operates.

The most recent publication regarding the use of IZON's nanopore system is by Robert et al. [97] where the study focuses on the application of IZON's qNano technology in detecting polystyrene nanoparticles and bio-functionalised nanoparticles. The work also shows the ability of qNano to measure a bimodal (which will be looked into in this research) and trimodal distribution of nanoparticles as well as the gating ability of this instrument.

Thus far, there are limited studies on the use of qNano for samples other than biological and inorganic particles. The main aim of this research is to explore the capability of the qNano technology in measuring a wider range of particles. A summary of the main aim of this research includes understanding capability of the instrument, i.e. its advantages and limitation; understanding the behaviour of the TPU apertures; how particles behaves in the standard buffer solution, and the information that can be extracted from the signal blockades of these particles - such as the particle diameter and surface charge. 
The qNano instrument has successfully detected biological molecules (bacteria and viruses) but not many studies have been done on inorganic and metal nanoparticles.

A variety of nanoparticles will be used in this research, which includes polystyrene, silica, and gold nanoparticles. Other noble metals such as silver and magnetic nanoparticles will also be investigated although not in as much depth as gold nanoparticles. These particles will be used to investigate the potential of the qNano to be used mainly as a particle sizing device that could substantiate data retrieved from other means of characterisation, such as transmission electron microscopy (TEM) and via the DLS method. All these findings will be crucial to establish the value of the qNano technology as a versatile tool for nanoparticle characterisation.

At the start of this research, there are a few limitations in the qNano that makes it challenging to detect nanoparticles, i.e. particles that have a mean diameter of less than $100 \mathrm{~nm}$. This includes the size of the nanopore (which is too large to detect the translocated nanoparticles), the low (10 kHz) bandwidth - which is the sampling rate of the signal-, and the driving force that is based solely on the electrophoresis to move particles across the nanopore. Experiments were designed to accommodate these limitations such as synthesizing gold nanoparticles (usually with sizes less than $50 \mathrm{~nm}$ ) with silica to make larger sized particles. However, throughout this research, product development by IZON makes detection of nanoparticles possible as will be discussed in this thesis. 


\subsection{EXPERIMENTAL SECTION}

This chapter will explain how a qNano system operates. qNano's user interface, IZON's buffer solution, and IZON's apertures will be discussed in detail. IZON's tuneable aperture allows the user to define the stretch of the aperture for each specific measurement. Consequently, the size of the particle blockades is relative to the stretch of the aperture, as shown in figure 2.1

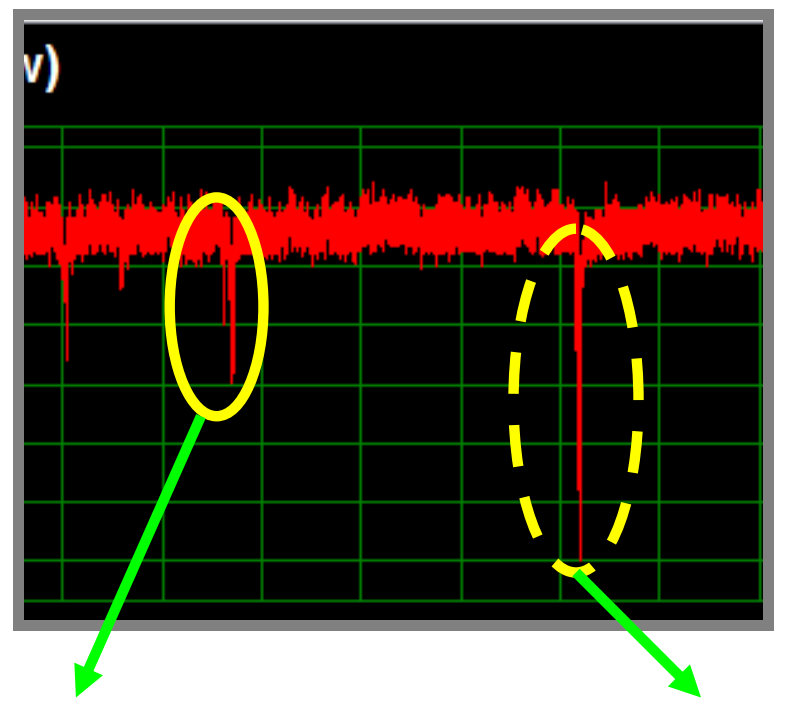

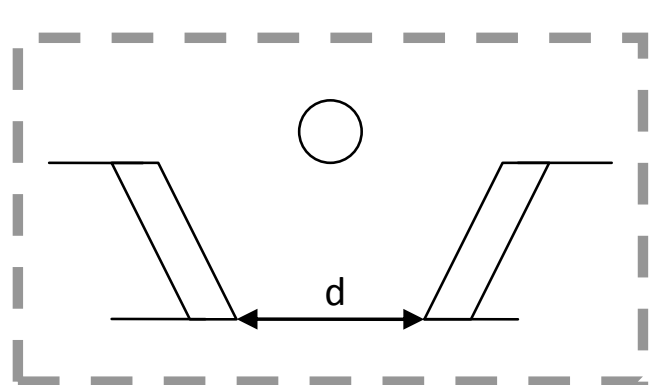

(a)

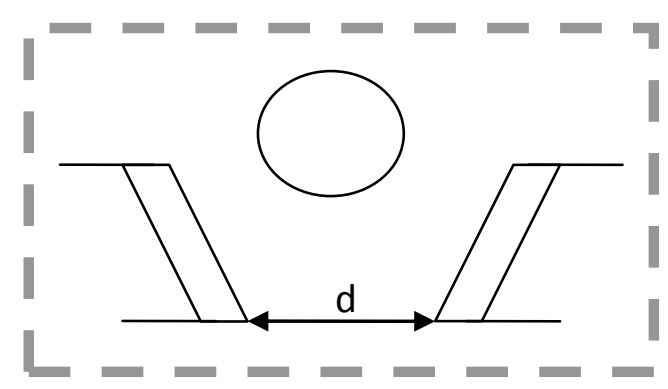

(b)

Figure 2.1: Illustrative description of blockade depth at constant stretch. (a) A small nanoparticles passing through the pore. (b) A large nanoparticles passing through the pore.

Figure 2.1 shows how the magnitude of blockade depth is relative to the stretch of the defined pore. With the pore stretch remaining constant, a smaller particle partially blocks the opening of the pore and a larger particle blocks most of the pore. This 
blockage prevents ions from flowing through the pore creating the 'resistance' [1]. This translates as a downward spike - or dip - in the signal as shown (by the yellow bubble) in figure 2.1. This type of blockade is called a resistive blockade and the depth of the blockade is indicative of the volume of the translocated particles. As volume has a cubic relationship to the particle's radius, this implies that the blockade depth relates to the diameter of the particle to the third power.

Conductive blockades, i.e. upwards spike, have also reported to be observed on this system [89]. Essentially a particle that moves through the pore is displacing a volume that is equal to the diameter of the particle. As stated before, each particle has a charge density associated to it $[17,89]$. If the charge density of the particle is more than the displaced volume of the buffer solution, then there is an increase in charge, which for the instrument, translates as a conductive spike.

Throughout this research, it was found that one of the main factors that affect the particle translocation count was the electrical noise level. A few things can be attributed to this including; noise from the main power source (the $50 \mathrm{~Hz}$ noise, an observable oscillation of the alternating current), noise from the electronics, thermal noise generated by the pore, as well as external disturbance such as hand movements over the fluid cell $[13,89]$. The latter will cause random spikes to appear in the signal window. Uram et al. [13] proposed that the pore could be assumed to behave like a resistor. One of the causes of resistance is due to the thermal movements of ions. This thermally induced noise is also known as the Johnson noise, and is reported to be the main cause for the noise level in the live trace signal [13]. Figure 2.2 shows an example of a noisy baseline current. 


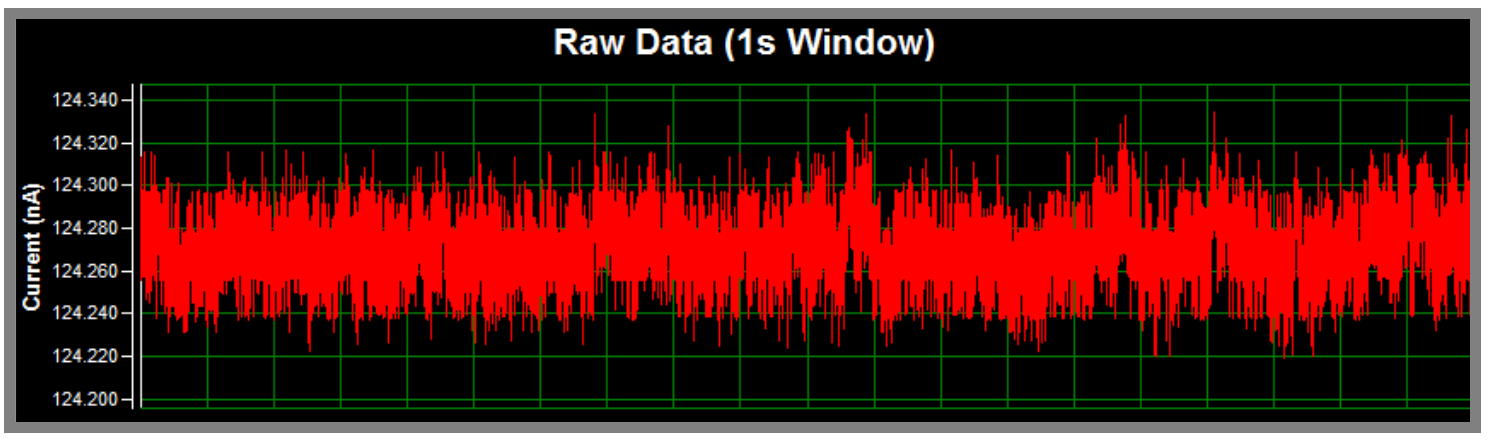

Figure 2.2: Screenshot of a noisy live trace signal. A stable baseline current would have a smooth line but as can be seen here an uneven baseline current is observed.

Ideally, the background noise should be kept at a minimum to ensure that all particle blockades - especially those in the nano-region - are detected and not drowned by the noise signal. The acceptable noise level is between 0.005 nA and 0.01 nA. Closer inspection of signals from example in figure 2.2 shows that the noise levels are between $0.05 \mathrm{nA}$ and $0.12 \mathrm{nA}$. Background noise detected also comes from external electromagnetic (EM) radiation. EM sources such as those given out by cell phones are detected by this instrument and examples of those signals are given in figure 2.3.
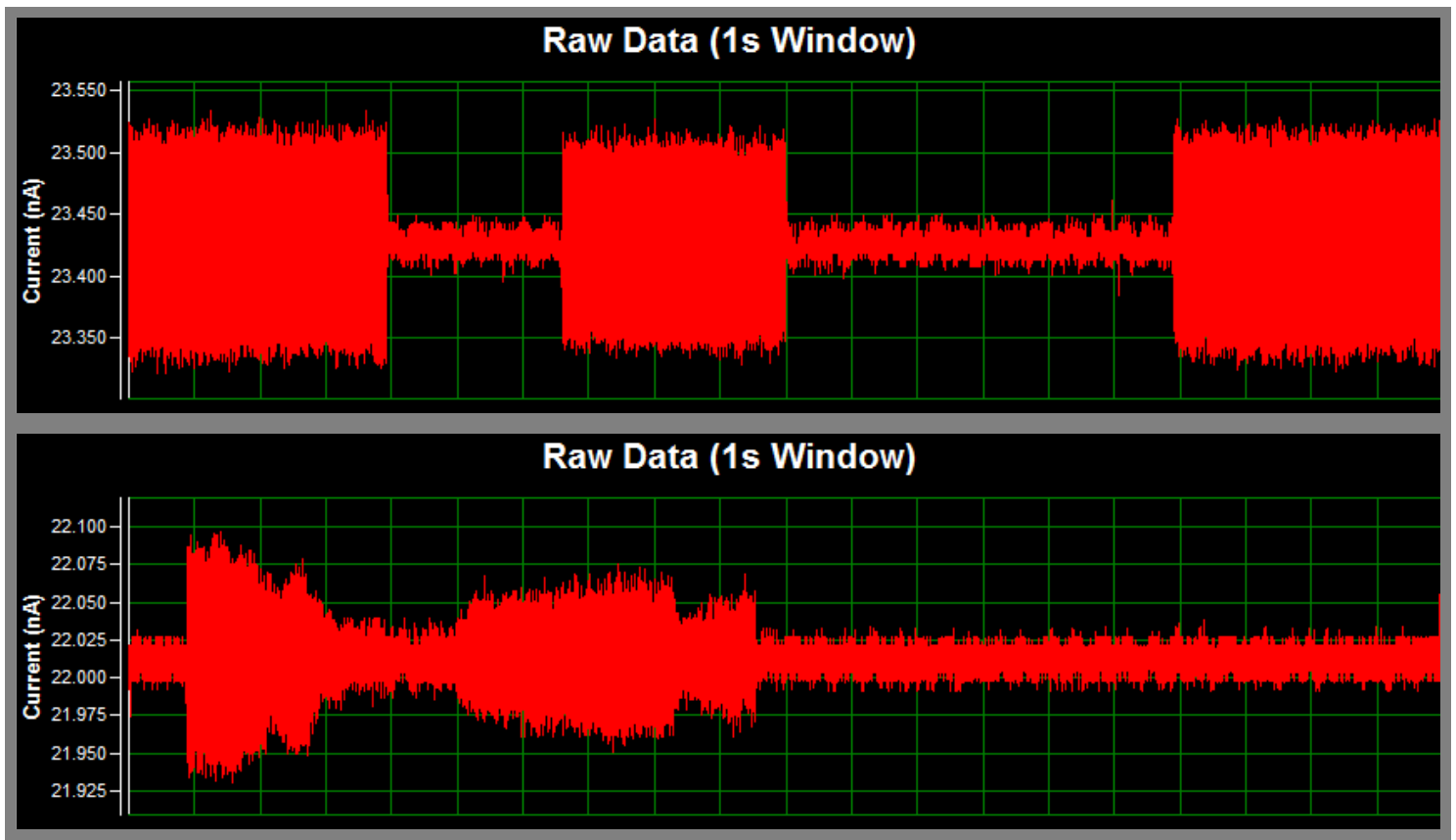

Figure 2.3: Screenshots of two different types of interference of external electromagnetic signals. An incoming electromagnetic radiation signal creates the 'block' pattern in the signal trace. 
Figure 2.3 shows examples of incoming electromagnetic radiation such as those given off by cell phones (the $1^{\text {st }}$ screenshot image in figure 2.3 ). These signals usually come in short pulses for only a few seconds. One measure to overcome this issue is by placing a metal cap over the top fluid cell. This metal cap acts as a Faraday shield. A Faraday cage (or shield) acts as a protector from external electromagnetic radiation that may induce electrical interference with signals from qNano [90].

One other factor that causes instability of a baseline current is the presence of air pocket or bubble in the fluid cell. Movements of the air bubble in the fluid cell causes a break in the conductivity hence the disturbance in the baseline current. Figure 2.4 shows two screenshots of the instances where current instability is observed.

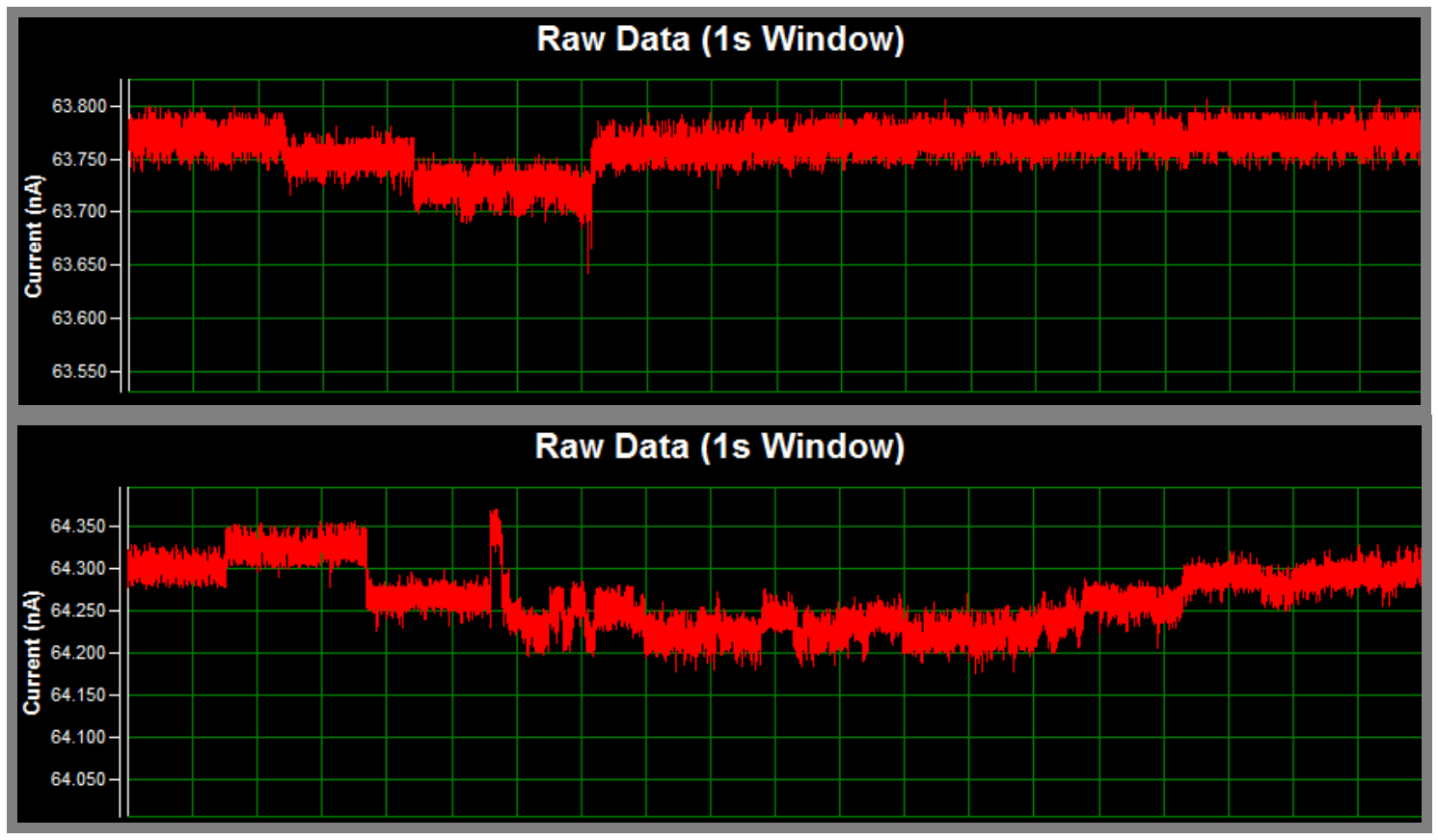

Figure 2.4: Screenshots of current instability due to disturbance in the fluid cell system such as air bubbles or a partially blocked pore.

As shown in figure 2.4 , the baseline current shows some instability (defined by the uneven current trace signal). Baseline instability can be fixed by simply refreshing the fluid cells i.e. dismantling the fluid cell and re-setting it up again allows current to run more smoothly. 


\section{1 qNano software - Izon Control suite}

IZON's user interface software - the IZON Control suite - is constantly being updated. Version 1.2 ( $V$ 1.2) software offers researchers the ability to calibrate the platform for particle concentration analysis. The algorithm for the data capture has also been improved to make 'losses' of particle detection down to a minimum.

In the next three chapters in this thesis, references to the components featured in the software will be made; therefore, it is important to state what information is retrieved from the major components on the software. Figure 2.5 shows the default user interface of the software.

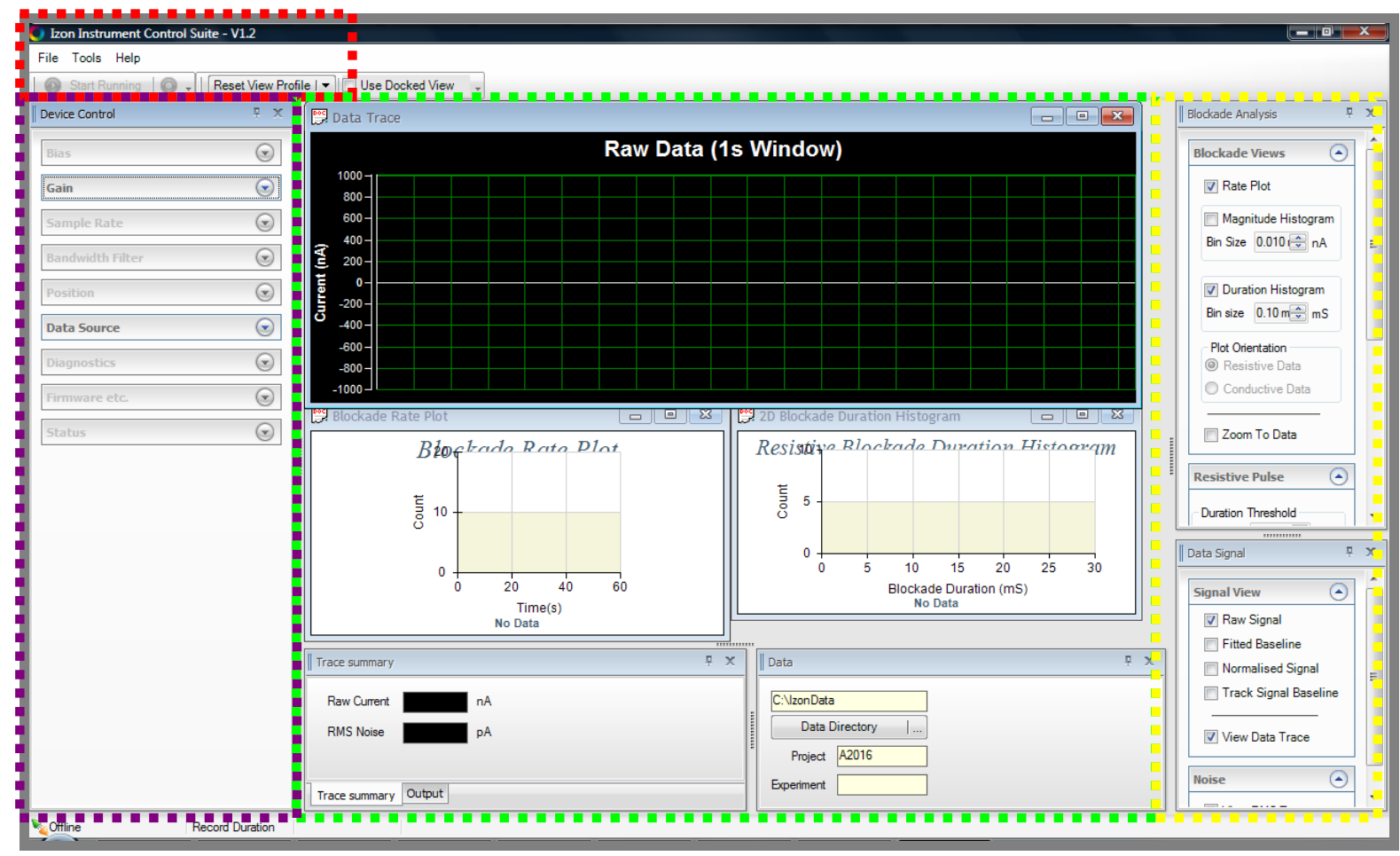

Figure 2.5: The default user-interface screen. This is the screen where user defines the settings of the measurement.

Figure 2.5 is the software's user interface on default settings. Components in the red dotted box contains the 'switch' to activate the data recording feature as well as a custom settings to view the user interface. A close-up of the red box is given in figure 2.6 . 


Izon Instrument Control Suite - V1.2
File Tools Help
$\mid\rfloor$ Start Running $|\bigcirc-| \mid$ Reset View Profile I - $\mid \square$ Use Docked View -

Figure 2.6: Data-recording and viewing options features.

The 'Start Running' button is the switch that allows the software to record measurements of particles going through the nanopore. A 'Reset View Profile' option allows the user to switch the observation window on the main page (figure 2.5) to a custom interface that is desired.

The purple dotted box is the 'Device Control' where the user determines the operational conditions of the instrument. Figure 2.7 details the settings and conditions that are available to be varied.

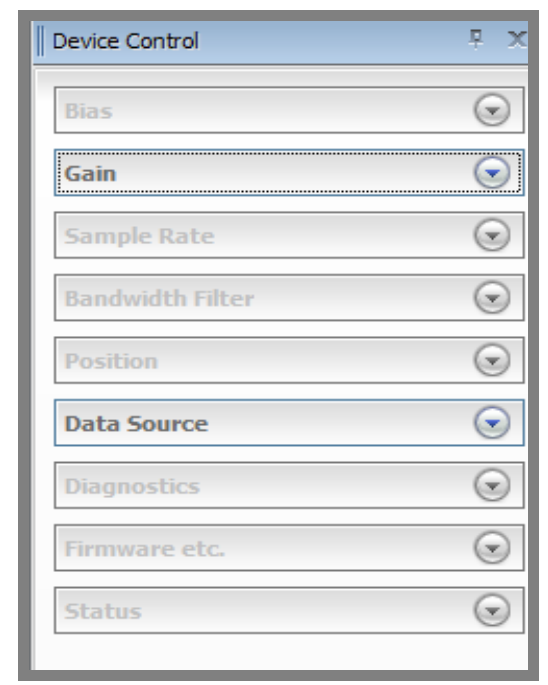

Figure 2.7: The device control window. Tabs that will be frequently used by the user is the 'Bias' tab where the voltage bias is set.

Figure 2.7 shows the window that is mainly used to adjust the settings and operational conditions of the instrument. Most of the tabs have default settings set by IZON. Usually, users only need to key their input in the 'Bias' tab and pay attention to the 'Position' and 'Data Source' tab. The 'Bias' tab is used to set the running voltage - the electrical potential difference between the upper and lower fluid cell. The 'Position' tab indicates how much the aperture is stretched and the 'Data Source' tab allows the user to either measure the data in real time or re-run captured data file. 
The green dotted box in figure 2.5 is the main window where particles translocation is observed as measurement data - such as its rate and counts - are tracked in real time. The next four figures show each screen tabs individually.

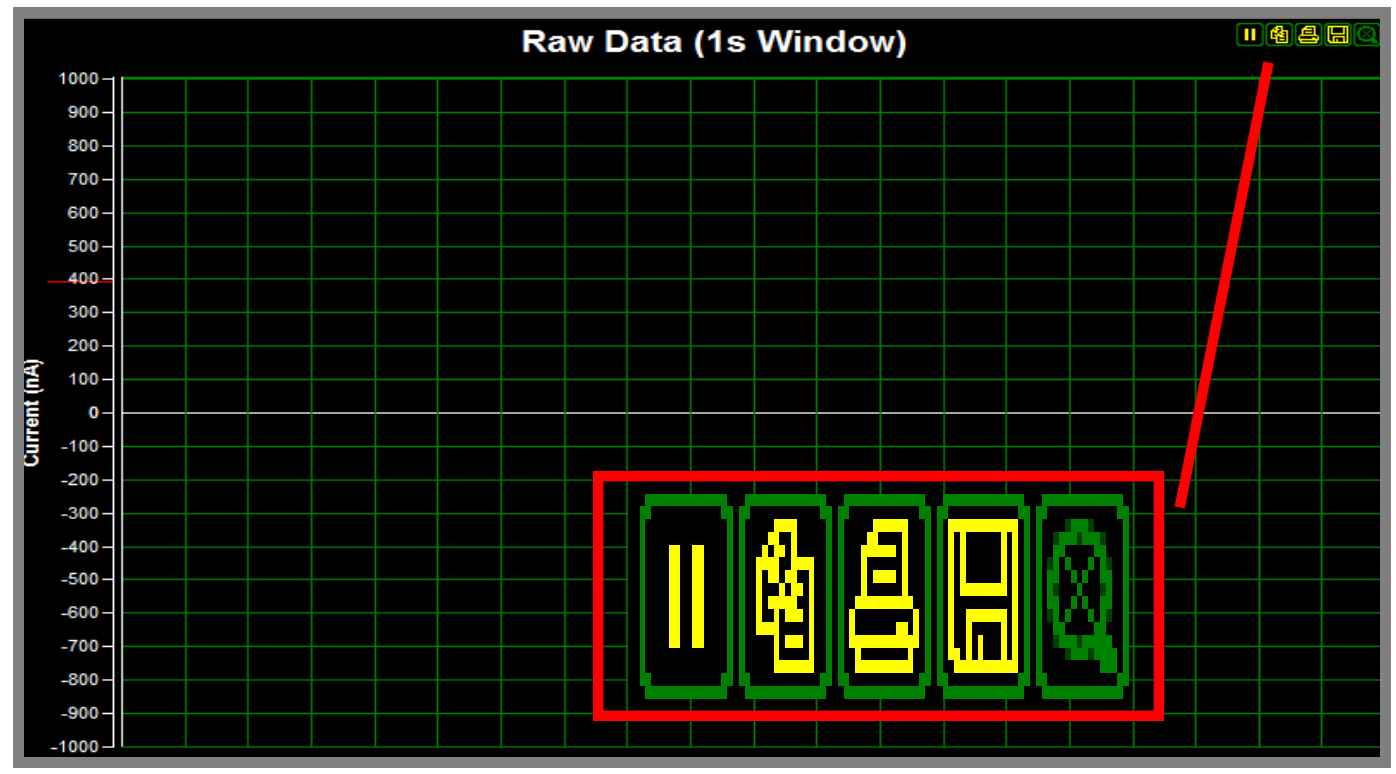

Figure 2.8: The main window to observe live trace signal of particles' translocation through the nanopore.

Figure 2.8 shows the main window of the user interface where raw data captured by the instrument are displayed. The raw data received is real time data and is plotted as a function of current over time. The top right hand of the window has options that enable the user to either pause the live data trace, print and save the signals, as well as a zoom-in function that allows a closer analysis to the data trace. A typical signal produced by the instrument is shown in figure 2.9.

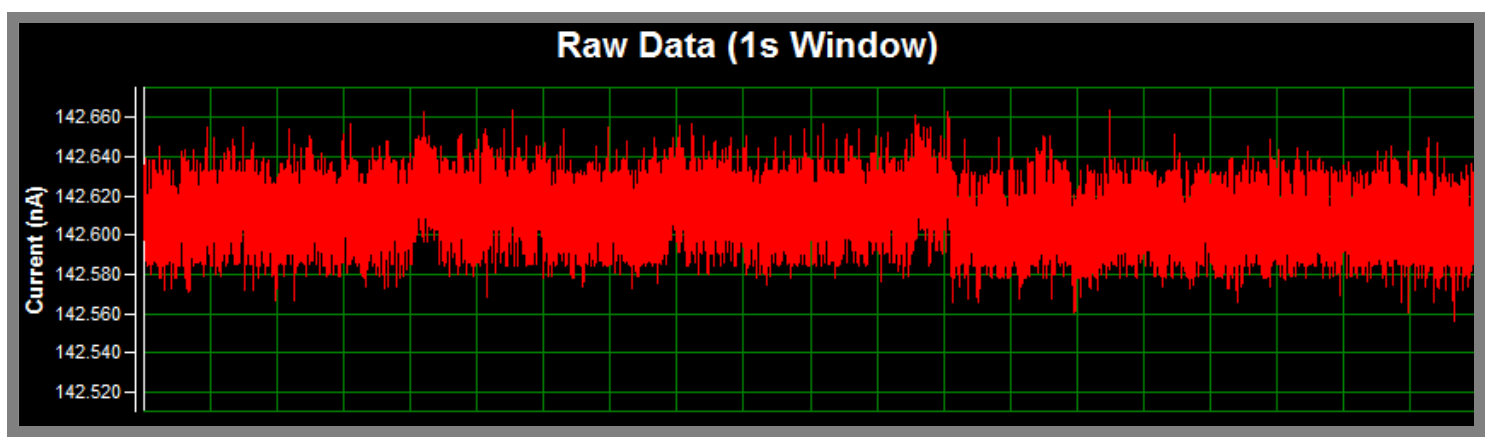

Figure 2.9: A typical current signal that runs through the aperture. It can be seen that the signals are stable with low noise and interference from other electrical instruments (which influence the instrument's sensitivity). 
Figure 2.9 shows the screenshot of a current signal that was recorded by the instrument. For a standard set up of the system, $75 \mu$ l of the buffer solution is pipetted on to the middle of the fluid cell. The aperture is then fixed on the stretching arms and the top fluid cell is then fixed on to the instrument. $40 \mu \mathrm{l}$ of the buffer solution is then delivered to the top fluid cell. The electronic component in the instrument (the Enigma 3 printed circuit board (PCB)) applies a fixed voltage through the nanopore and measure the current that runs through the pore at a rate of $50 \mathrm{kHz}$. This current forms the baseline current, which is calculated by averaging all of the current measurements over a set number of samples. This set number of samples is called the 'Analysis Window', which can be defined by the user. The level of background noise (the root mean square (RMS) noise) is also calculated over the analysis window $[89,99]$.

A block in the nanopore creates a resistance to the current flow and this cause deviation in the baseline current. If this deviation surpasses $8 x$ the level of the RMS noise, this deviation will be registered as one 'Blockade' event. For each blockade event, the information that the system record include the maximum deviation from the baseline current - 'Blockade Magnitude'; the time that the current deviation occurs - 'Blockade Duration'; the time that the current exceeds a threshold defined at half the blockade magnitude - 'Duration Full Width Half Maximum (FWHM)'. The last feature is used to normalise measurement for blockade of different sizes [89, 98, 99].

Signal analysis of the live trace data is also produced and the information is given such as in figures $2.10 \mathrm{a}$ and $2.10 \mathrm{~b}$. 


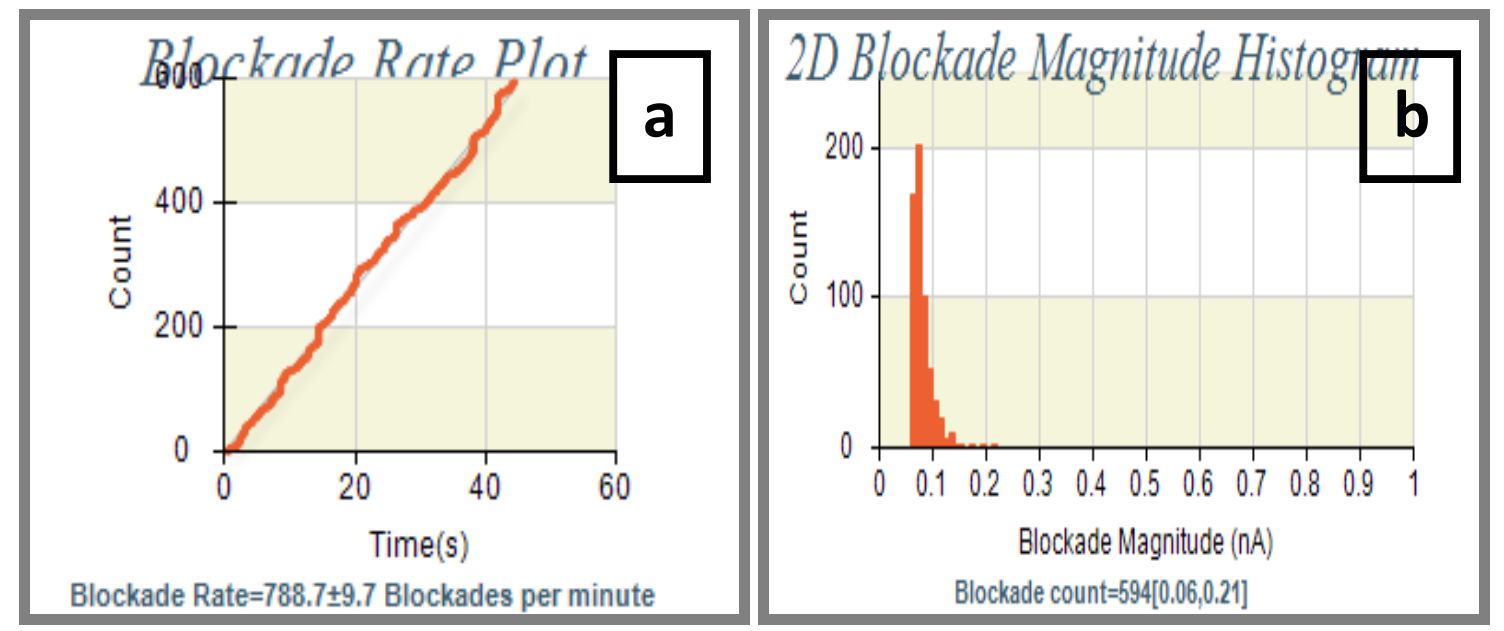

Figures $2.10 a$ and $2.10 \mathrm{~b}$ : Window on signal analysis of the live trace. The window serves as a tool to observe the rate particles are going through the pore and its distribution. A stable measurement gives a linear plot in the 'Blockade Rate Plot' window (2.10 a).

Figure 2.10 a is the window that shows the rate of the particles going through the pore in real time. A stable measurement would produce a blockade rate plot that is linear. A disturbance in the live trace - produced by either pore blockage or noise signals would generate a 'jump' in the blockade rate plot which signifies that the measurement is unstable. Figure $2.10 \mathrm{~b}$ on the other hand is the histogram distribution of the blockade magnitude as it goes through the nanopore. A duration histogram of the particle blockades can also be viewed by customising the interface to have a duration histogram instead. 


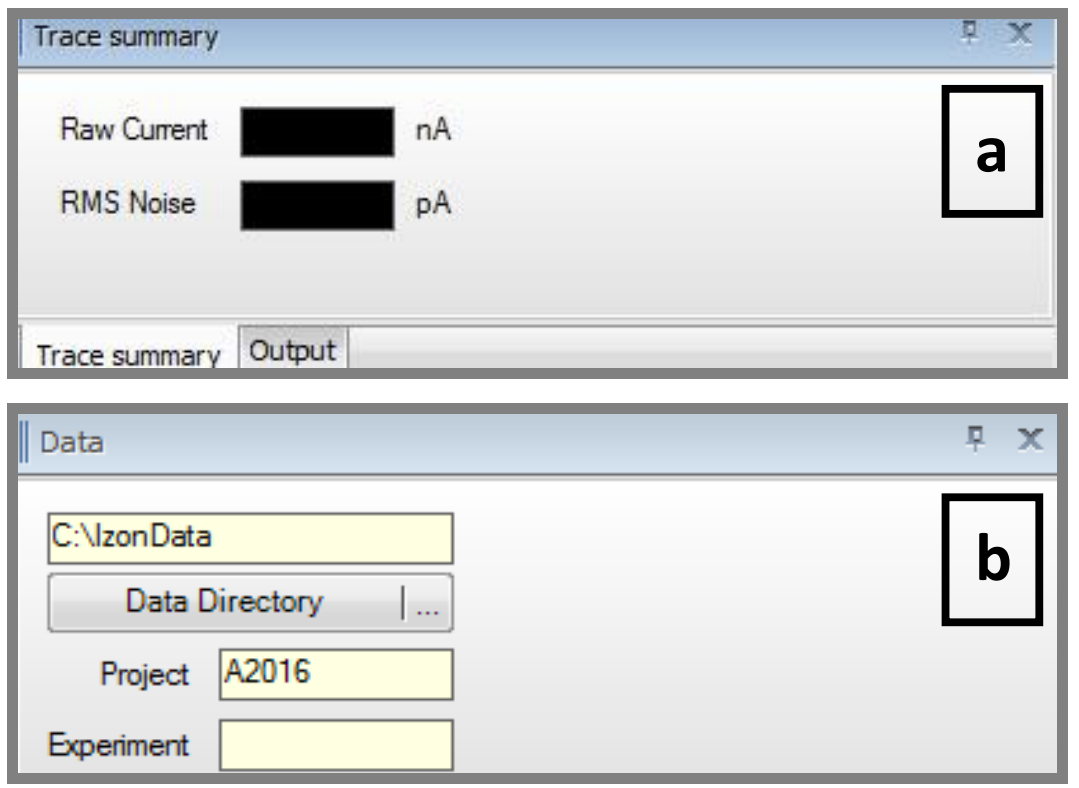

Figures $2.11 a$ and $2.11 b$ : Details of the live data trace. This feature allows user to view the exact numerical value of the raw current and noise level (2.11 a) as well as keeping records of the measurements taken on the instrument (2.11 b)

Figure 2.11 a shows the details of the baseline current as well as the background noise level. It is crucial to note the value of background noise as this will determine the magnitude threshold settings. It is also important to have the correct magnitude threshold so that data recorded by the software is of particles blockades only. Figure $2.11 \mathrm{~b}$ is the information for data storage. It allows the user to systematically keep track of the recorded data files and to define the measurements to avoid confusion. Figure 2.12 shows the data file for the recorded measurement with V1.2 software.

\begin{tabular}{|c|c|c|c|c|c|c|c|c|c|c|}
\hline & A & $B$ & C & D & $E$ & $\mathrm{~F}$ & G & $\mathrm{H}$ & 1 & $J$ \\
\hline 1 & StartTime & Duration & Duration FWHM & Magnitude & Baseline & Bias & DeltaX & SignaIRMS & Forced Resets & Blockade Orientation \\
\hline 2 & 0.37986 & 1.06 & 0.58 & 0.1515 & 93.553 & 0.5 & $\mathrm{NaN}$ & 0.01 & & Resistive \\
\hline 3 & ก 38กah & ח 26 & 142 & م ח586 & 025528 & 05 & $\mathrm{~N} a \mathrm{~N}$ & 1 & & Recictive \\
\hline
\end{tabular}

Figure 2.12: Data file for recorded measurements. The data file consists of all the information extracted by the software from the instrument for post-hoc analysis.

Figure 2.12 shows a sample of the details of particle blockades that was recorded in a measurement. The software gives the following information of the particle blockades: Start time - the time at which a blockade was recorded; the depth of the blockade magnitude; the current at which the blockade events takes place (Baseline Current); voltage used (Bias); aperture stretch (Delta X); and a 'Blockade Orientation' that states 
if the particle blockade is resistive or conductive. The forced reset column is a feature that allows analysis process to be restarted when signal from the instrument becomes corrupted for any reason. Therefore, this column indicates if any forced resets were done as measurement was taken. Every time the software restarts itself, the forced resets increase by a value of 1 . Therefore, the best measurement would be if there were no forced resets happening during a measurement [89].

As stated before, it is important to determine the magnitude threshold of the particle blockade. These settings can be adjusted using the window given in the yellow dotted box in figure 2.5. A close-up of the yellow box is given in figure 2.13.

\begin{tabular}{|c|c|}
\hline \multicolumn{2}{|c|}{ Resistive Pulse } \\
\hline \multicolumn{2}{|c|}{ Duration Threshold } \\
\hline & $0.03 \triangleq \mathrm{mS}$ \\
\hline $\operatorname{Max}$ & $5.00 \stackrel{A}{\vec{v} S}$ \\
\hline \multicolumn{2}{|c|}{ Magnitude Threshold } \\
\hline & $0.060 \stackrel{\wedge}{\vee} \mathrm{nA}$ \\
\hline & $1.000 \stackrel{\wedge}{\vee} \mathrm{nA}$ \\
\hline D An & lyze \\
\hline
\end{tabular}
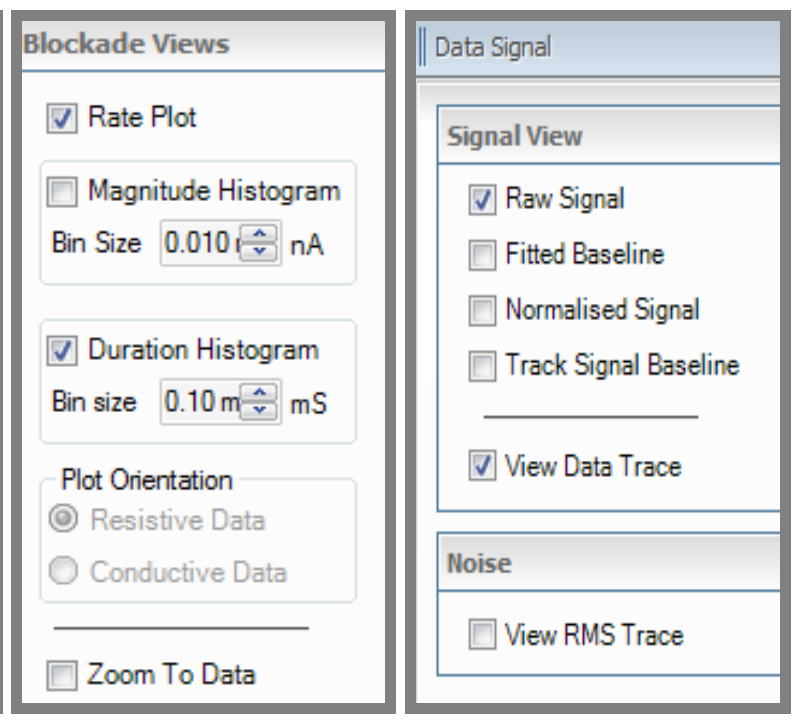

Figure 2.13: 'Blockade Analysis' window of the interface gives user the ability to change the settings to suit the requirement of the measurements for optimum results.

Figure 2.13 shows the blockade analysis tabs that are important to users. The 'Resistive Pulse' tab allows users to define the settings for data recording of the particle blockades. The 'Duration Threshold' has a minimum and maximum time where particle blockades will be recorded. Taking an example from figure 2.13 , this means that if the live trace is recorded, only data that goes through the pore at a minimum time of 0.03 $\mathrm{ms}$ and a maximum time of $5 \mathrm{~ms}$ will be recorded. Particles that translocate faster that $0.03 \mathrm{~ms}$ and slower than $5 \mathrm{~ms}$ will be ignored. 
The same concept applied for settings for the 'Magnitude Threshold'. A blockade will be recorded to between the set lower and upper limit. In the next few chapters, there will be mention of the magnitude threshold and the notation used throughout this thesis will be $[x, y]$ where $x$ represents the lower limit and $y$ the upper limit -i.e. [0.06, 1] $\mathrm{nA}$ (values taken as an example from figure 2.13). This means that if a blockade has a magnitude less than $0.06 \mathrm{nA}$ or more than $1 \mathrm{nA}$, it will not be registered in the data file. The settings of the 'Magnitude Threshold' have found to be most important as inaccurate lower or upper settings can give a false recorded data points - as it will account for the background noise as well - and upset the blockade counts of the measurement tremendously.

The software also allows the user the option to view the live trace signal in a 'Normalised Signal'. This normalised trace calculates the baseline trace as zero and takes into account only the blockade size detected by the instrument. This function is especially important to determine the settings of the blockade magnitude threshold. If the noise level is known, the lower limit of the magnitude threshold can be correctly set. A threshold too low might count the noise levels as blockades and a threshold too high will overlook actual particle blockades. Figure 2.14 shows an example of the difference between the raw signal window and a normalised signal window. 

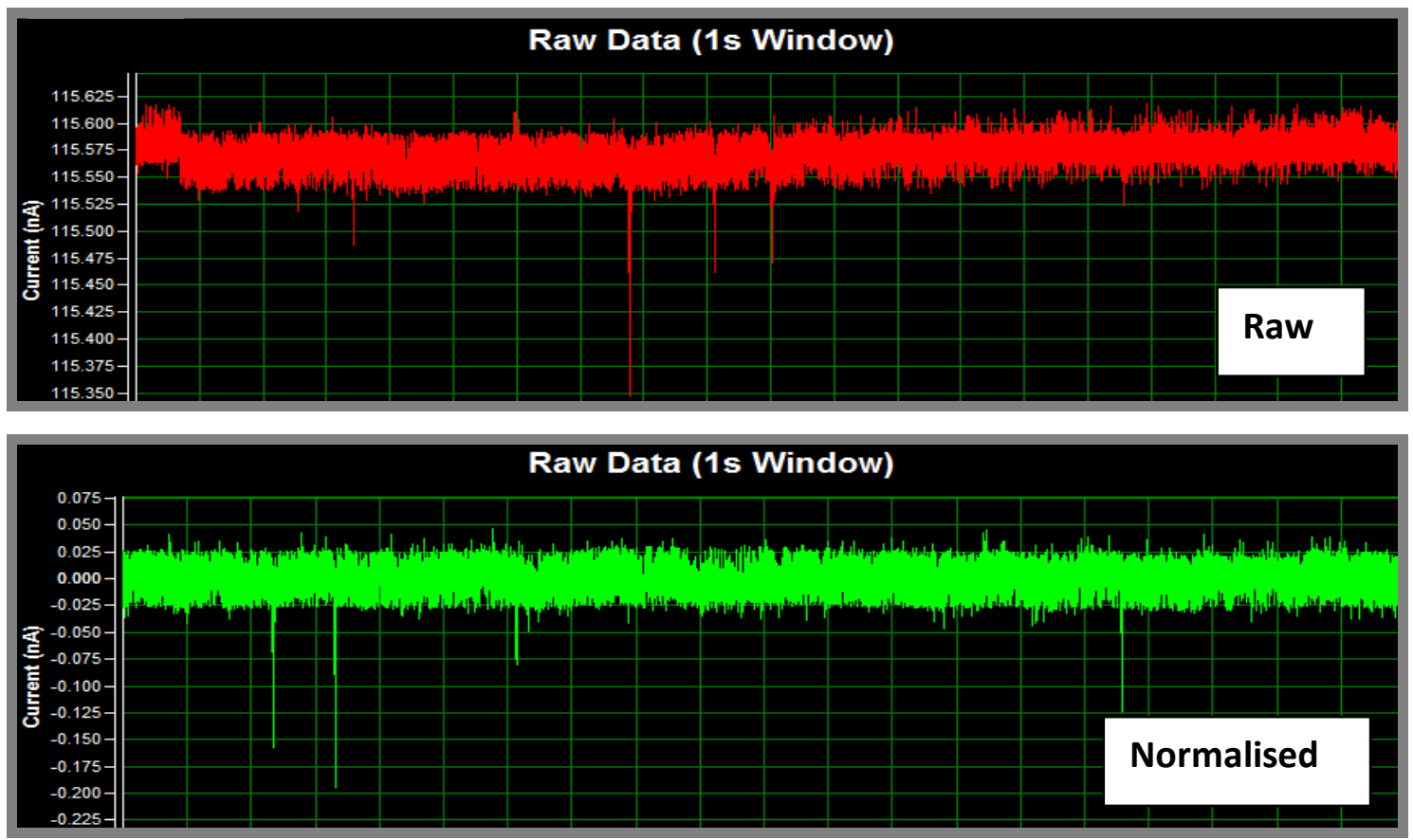

Figure 2.14: Signals window comparison between raw signals and normalised signals. The current for a normalised trace signal is reduced to approximately $0 n A$ while maintaining the magnitude for the blockade size.

Figure 2.14 shows the two main observation window of the blockade signals on the software. The default view is the 'Raw' data trace (in red) and the green data trace is from the 'Normalised' view. As can be seen, the raw view gives a current reading of approximately $115.5 \mathrm{nA}$ and the normalised view shows a reading of zero. However, particle blockades are still detected. This normalised view keeps data integrity and information on particle blockades intact while eliminating redundant information. The advantage of having a normalised signal is that it makes estimation of blockade size much easier but also, any step down of current due to pore blockages for example will not be recorded.

\subsection{Thermoplastic polyurethane (TPU) apertures}

IZON's apertures are made from thermoplastic polyurethane (TPU). TPU has many advantages, which include resistance to physical scratching and chemical changes; structural flexibility; and compatibility with biological systems [80]. An image of IZON's specimen cruciform is given in figure 2.15 . 
The cruciform arm. The holes at the end of the arm are used to set the cruciform in place on the instrument.

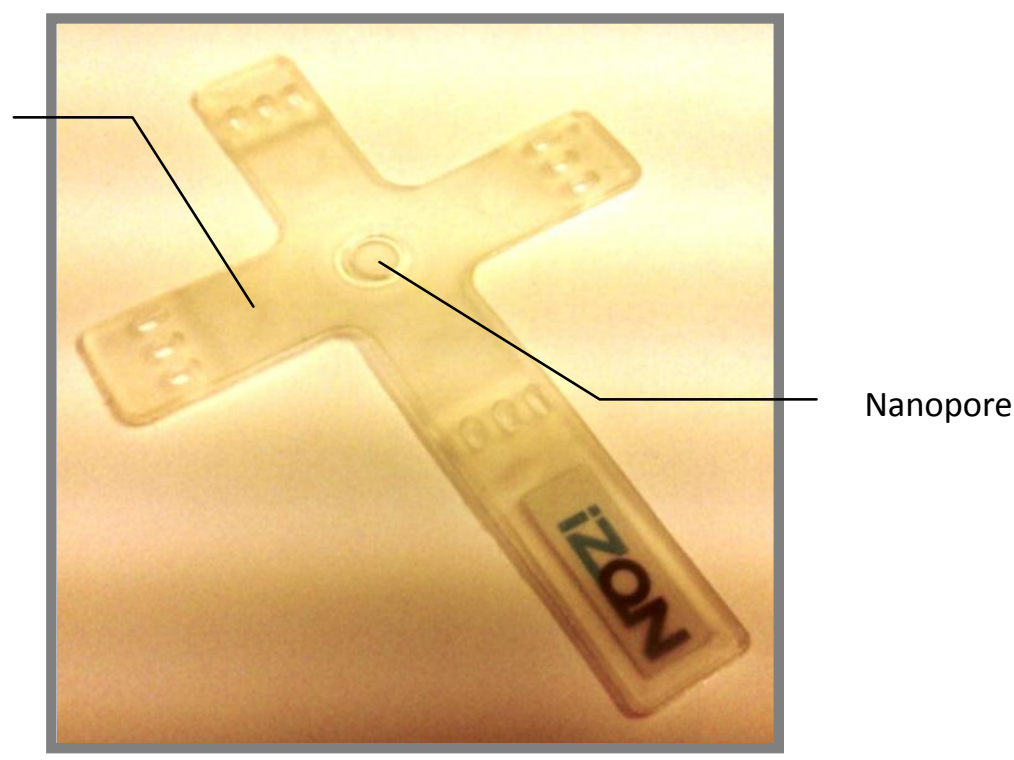

Figure 2.15: IZON's specimen cruciform. Approximate scale is $1: 0.7 \mathrm{~cm}$

Figure 2.15 shows an image of IZON's specimen cruciform. The nanopore is centred in the middle of the cruciform. The cruciform arm extends out from the nanopore and is used to hold the aperture in place. This is done by fixing the aperture to the stretching arms of the instrument via the holes at the end of the cruciform arm. Stretching the pore on both $\mathrm{X}$ - and $\mathrm{Y}$-axis ensures that the nanopore is stretched evenly.

The pore of IZON's aperture is fabricated by using an etched tungsten needle to puncture the centre of the aperture on the cis side and out on the trans side as discussed in depth by Sowerby et al. [6]. The amount of force used to puncture the hole is auto-controlled and ultimately, determine by the size and shape of the hole. The needle used to puncture the hole however wears out over time and the change of needles may mean that the shape or size of each pore is varied in each aperture [86, 89]. The nature of the needles produces the aperture that is designed in a conical shape. The schematic diagram of this is given in figure 2.16. 


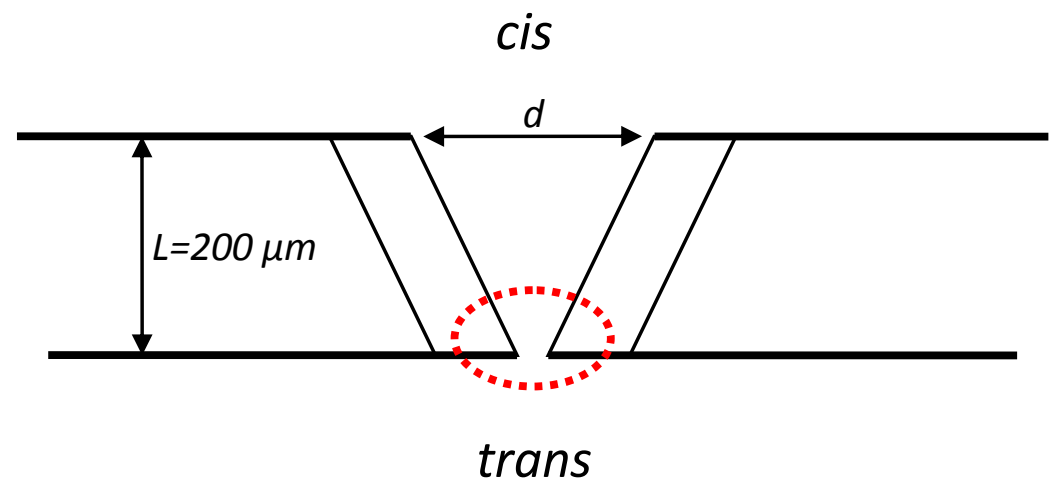

Figure 2.16: A schematic diagram of IZON's apertures. Puncturing of the membrane happens from the cis side. The needle used in hole-making is attached to a computer controlled actuator.

Figure 2.16 shows the schematic diagram of a typical nanopore. Due to the conical shape of the hole, the electric potential that runs through the pore creates a field that is most concentrated at the tip of the pore as shown in the red bubble in figure 2.16 [2, $12,33,86]$. This is an important feature of the hole as it reduces the effective length of the pore [2] and allows detection of individual particles. The concentrated electric field can be exploited and be used to study on areas such as separating strong and weakly polarisable nanoparticles as well as DNA stretching [12]

Consider if the pore is cylindrical in shape, as demonstrated in figure 2.17.

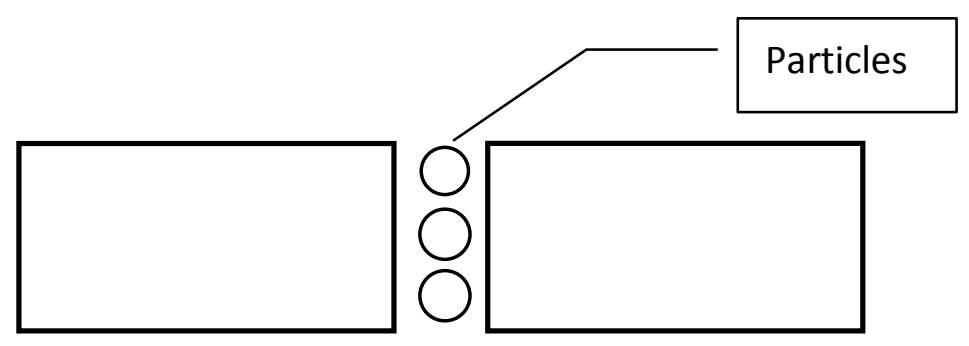

Figure 2.17: A diagram of a cylindrical pore

Figure 2.17 shows a representation of an aperture with a cylindrical pore. The symmetry of the shape of a cylindrical pore means that there is no difference in the concentration of electric field on the cis and trans side of the aperture. Using figure 2.17 as an example, if the particles goes through the pore continuously, these particles will block the pore for a period of time and the signals shown on the live trace will be such as in figure 2.18 . 


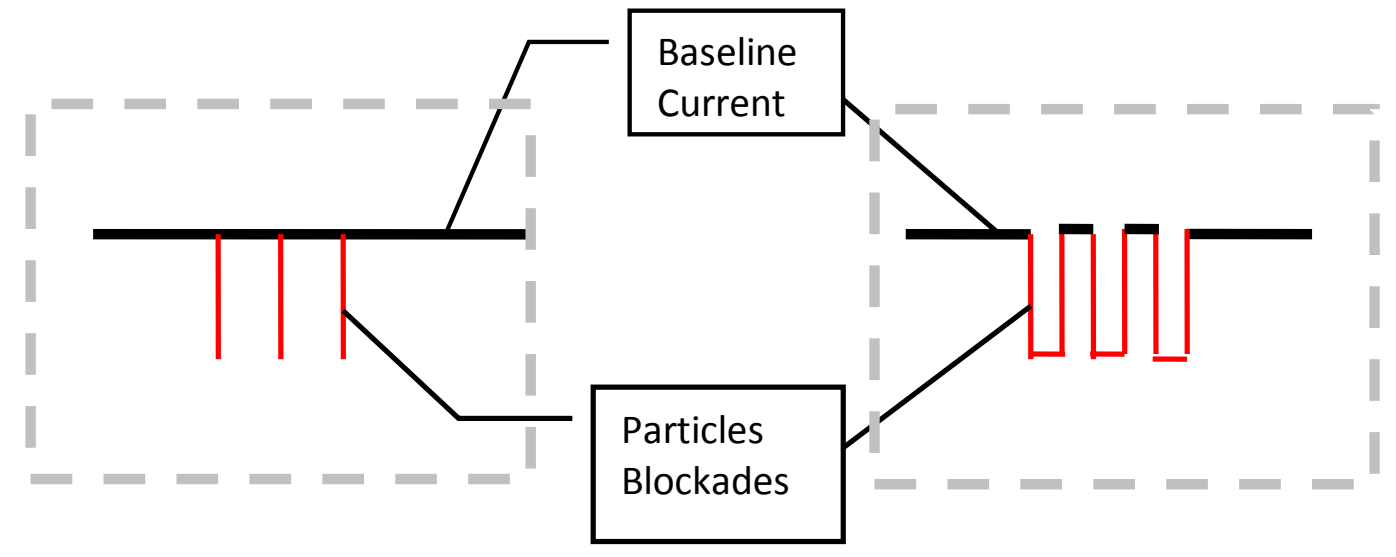

(a)

(b)

Figure 2.18: Expected signals from blockades with (a) A conical nanopore and (b) a cylindrical nanopore. Signals from the cylindrical pore give a stepped blockade trace.

IZON's conical nanopore can be thought of as a short and narrow cylindrical pore (equivalent to the small end of a cone) where electric field that pass through the pore concentrates at the tip of the nanopore on the trans side as shown in figure 2.16. The advantage of a short and narrow cylindrical nanopore compared to a long and wide cylindrical nanopore means that a smaller resistance is achieved.

One of the bases of IZON's marketing approach is the fact that the aperture is resizable. This allows a vast opportunity for research in other areas especially in biological research as well as ion channel studies [1-26]. Throughout this thesis, the term $(\Delta \mathrm{XY})$ will be used to indicate how much the nanopore is stretched. This term signifies the difference of the final aperture stretch in comparison with the initial aperture stretch. The stretch is measured by placing a vernier calliper on the outer side of the stretching arms on either of the X- or Y-axis. By default, IZON's initial aperture stretch $\left(X Y_{0}\right)$ is fixed at $41 \mathrm{~mm}$ [89]. Rotating the stretching mechanism will increase or decrease the stretch of the nanopore depending on which way the mechanism is rotated. The final stretch $\left(X Y_{f}\right)$ at which measurements are taken are recorded. The size of the pore stretch, $\Delta \mathrm{XY}$, is taken to be the difference between the final and initial stretch and this gives an indication on how much the nanopore is stretched. The actual size of the hole of the nanopore varies depending on the fabrication conditions of the nanopore, which ultimately determine how large the pore diameter is. 
Having the role of many static pores and the ability to unclog pores are examples of the advantages of a tuneable nanopore. This feature is extremely useful as it gives the apertures a longer shelf life compared to a static pore [86]. However, one of the limitations of the TPU apertures as any other viscoelastic material is that they experience a softening of the material under stress after initial stretch. This leads to the occurrence of Mullins effect, which is a phenomenon that shows a repeatable trend of the stress-strain curve after repeated stress-cycling. This is true only if the material is not stretched past its maximum stretch during cycling [80-84].

TPU is mainly made up of two segments; the hard segments - which are key in determining its mechanical properties-, and soft segments. These form a two-phase microstructure $[80,81]$ as represented in figure 2.19.

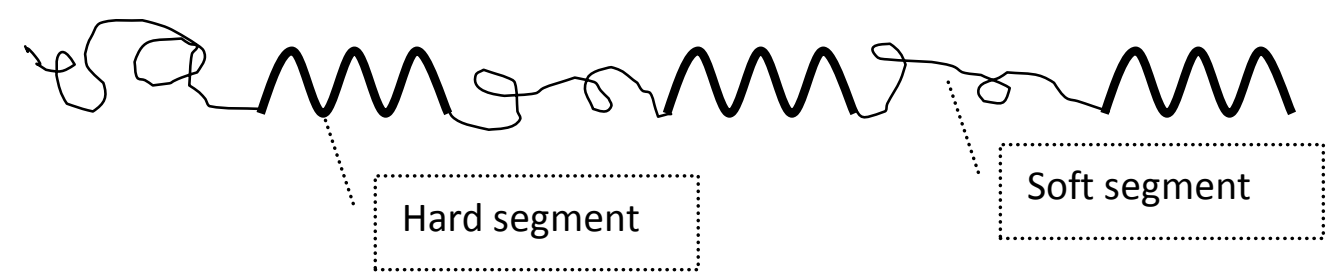

Figure 2.19: Illustration of the structure of thermoplastic polyurethane (TPU).

Stretching of the aperture over time causes irreversible changes in the microstructure. These changes occur because of the clashing properties between the two segments. The hard segment is made up of polar materials, which has the ability to form hydrogen bonds with each other, while the soft segments form amorphous domains. [80-82]. This theory was developed by Mullins and Tobin [85] who suggested that the softening is due to the misalignment of hard and soft segments between chains as aperture is stretched/strained $[80,81]$.

Boyce and Qi [80] reported that TPU shows a strong hysteresis and the stress-strain profile of TPU depends largely on the time the polymer is stretched as well as repeated softening. The softening of a material depends on the history of the polymer, in this case the aperture. Boyce and Qi [80] also reported that after the first stretching or loading of stress to the material, the softening will 'slow down' and stabilize and only 
limited softening occurs after a period of usage. This stabilization is caused by a few factors, including the hydrogen bond breakage in the hard domain as well as the interaction that forms between the soft and hard domain $[80,81]$. The issue of softening, however, can be rectified by increasing the hard segments as it acts as a cross linker between the segments and reinforcing it with very stiff particles, [82, 84].

\subsection{IZON's Standard Buffer Solution (SEB)}

IZON provides a buffer solution in its starter kit as a standard procedure for all its customers. Figure 2.20 shows the buffer solution supplied.

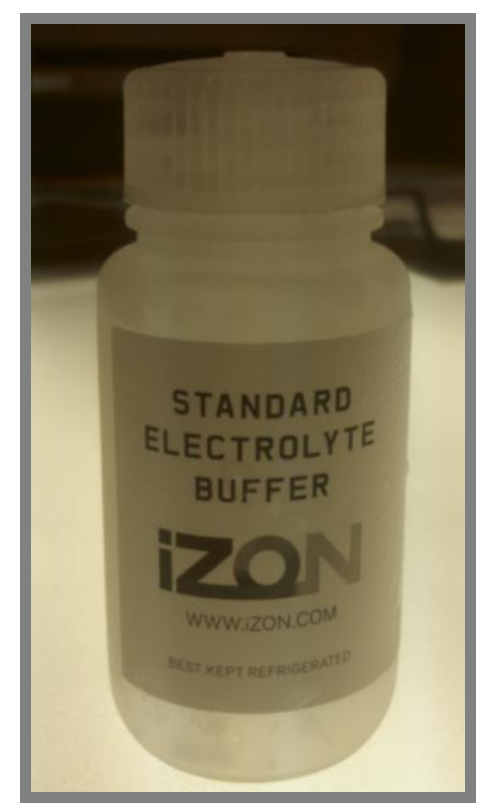

Figure 2.20: IZON's standard buffer solution

IZON's buffer solution comprise of four different components which are $100 \mathrm{mmol}$ of potassium chloride $(\mathrm{KCl})$ which acts as an ionic carrier, $0.01 \%$ of Triton-X 100 as a wetting agent, $10 \mathrm{mmol}$ of tris (hydroxymethyl) amino methane (abbrev -Tris) used as the buffering agent which maintains the solution at $\mathrm{pH}$ of 8 , and $3 \mathrm{mmol}$ of Ethylenediaminetetraacetic acid (EDTA) - a chelating agent to chelate any divalent ions such as $\mathrm{Ca}^{2+}$ and $\mathrm{Mg}^{2+}$ which causes aggregation if present in the solution. The $\mathrm{pH}$ of this buffer solution is adjusted to a value of 8 and is filter sterilized with a $0.22 \mu \mathrm{l}$ Millex GS MF-Milipore MCE Membrane filter. A pH value of 8 is chosen as it is found to 
be sufficient for the solution to 'charge up' the particles so that it can be moved through the nanopore [89].

The choice of the buffer solution that is currently used originates from its use with biological molecules [89]. However, this buffer is not specifically designed to work solely with biological molecules. There is also no concrete reason why it cannot be used with non-biological molecules hence, its choice to be used as the default buffer solution. Other electrolytes can also act as a buffer solution such as Phosphate Buffered Saline (PBS), seawater, and as will be discussed in this thesis in chapter 4, a sodium citrate buffer. However, because IZON uses a specific recipe for the buffer to test its system and all its apertures, a different buffer solution would affect other factors like the charge on the particle, and this in turn would make data comparison a challenge. In essence, any solution that has ionic carrier is suitable to act as a buffer solution.

\subsection{Variable Pressure Module (VPM) - A current development}

This new addition to the qNano technology uses a water-based manometer to give added pressure $(0-1.5 \mathrm{kPa})$ to the system. Some of the advantages of this new addition to the technology include the ability to detect charged and uncharged particles, concentration analysis, extended concentration range sensitivity, all of which gives an access for advanced research using qNano [88]. Figure 2.21 shows the VPM as attached to qNano. 


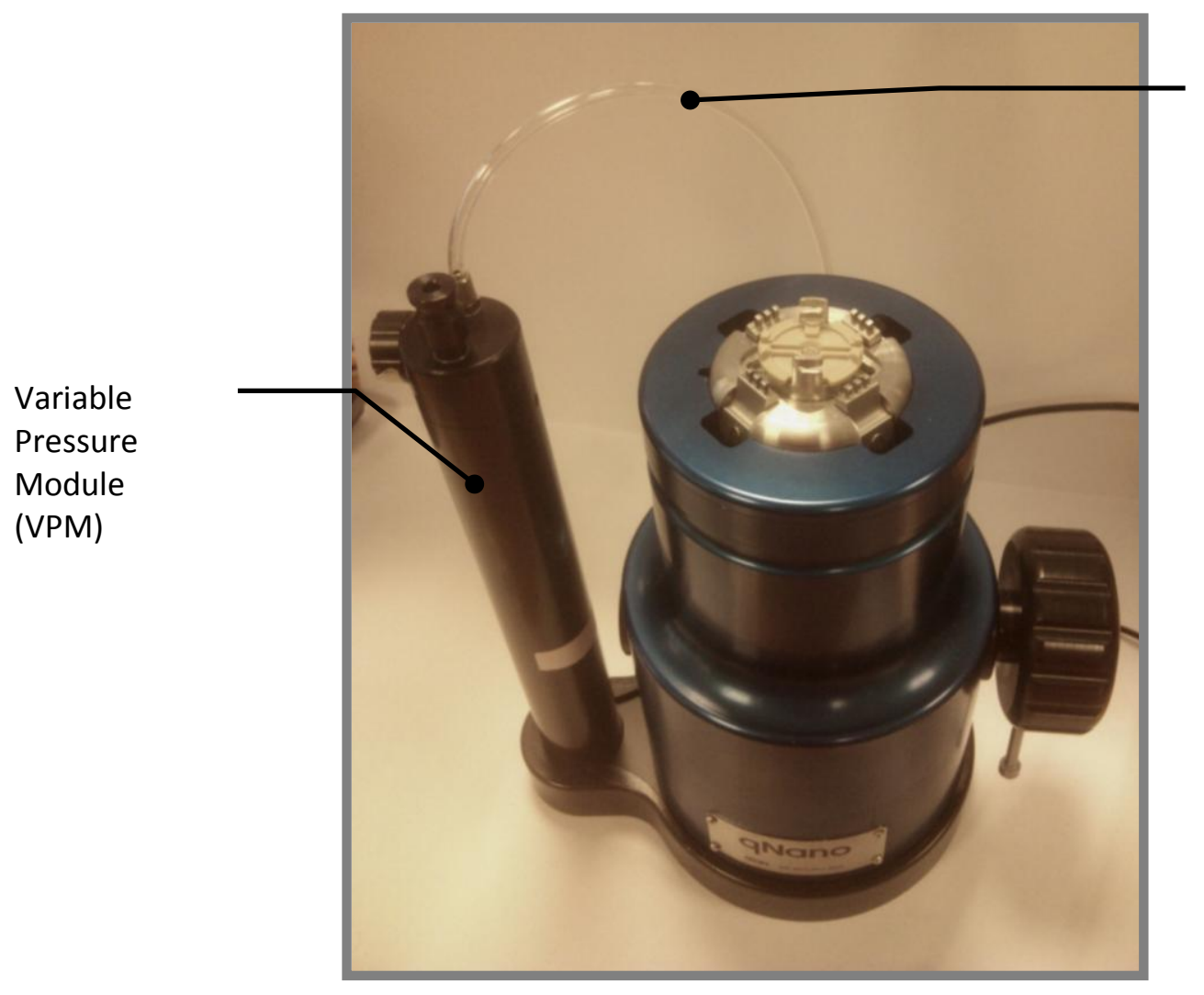

Connecting tube between the VPM and the instrument

Figure 2.21: The variable pressure module (VPM) is the long black attachment that accompanies the qNano. The end of the clear tube that is attached to the VPM connects to qNano's upper fluid cell.

The pressure system is a good tool to use for particles with an infinitesimal or nonexistent surface charge. Running conditions such as the aperture, duration threshold as well as the amount of pressure used may have to be tuned in order to get good data and results so that the workings of the system with the VPM can be understood. 


\subsection{MEASUREMENTS OF POLYSTYRENE PARTICLES}

\subsection{Introduction}

This chapter will explain how qNano technology works and highlight its ability to detect nanoparticles in a variety of settings. It will begin by detailing the stability of IZON's aperture - how the aperture's current profile changes as it is used over a period of time

- which will demonstrate the occurrence of aperture softening and the Mullins effect. This chapter will then describe the protocol used to optimise the system for accurately detecting particles going through the nanopore. This protocol involves the adjustment settings of the aperture stretch as well as manipulating setting conditions on the software. The results from this research will show that for each aperture, there is a working stretch range that will give the true representation of particle translocation through the nanopore. A section on the capability of the instrument to resolve different diameters and species of the polystyrene particles will also be examined. In this research, the instrument's capability to resolve two sizes of polystyrene particles, i.e. a bimodal distribution, will be discussed. Section 3.2.3 will discuss the difference in signals between polystyrene particles and carboxylated polystyrene particles. The results detailed in this section will show that un-functionalised polystyrene particles travel through the pore quicker than those with a functional group attached. In addition to this, $70 \mathrm{~nm}$ polystyrene particles were also detected, which exhibit the instrument's ability to detect particles in the nano-region.

\subsubsection{Setup of the qNano measurement system}

IZON uses carboxylated polystyrene particles purchased from Distrilab as a means to characterise its apertures. The particles IZON supplied come in the following diameters; $70 \mathrm{~nm}, 100 \mathrm{~nm}, 200 \mathrm{~nm}, 400 \mathrm{~nm}$, and $780 \mathrm{~nm}$. An example of the quality of the polystyrene particles used is shown in Figure 3.1. 


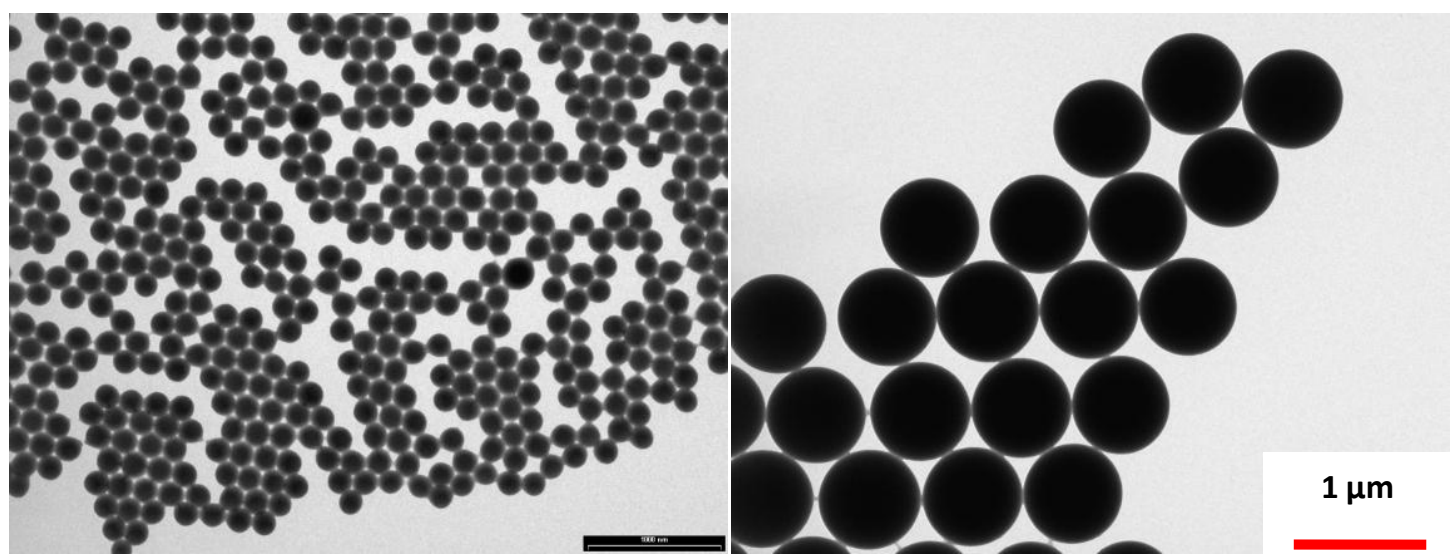

Figure 3.1: Example of the carboxylated particles with diameters $220 \mathrm{~nm}$ and 780 $\mathrm{nm}$. All images are at the same magnification. Photo courtesy of IZON Science.

In this study, polystyrene particles were used to demonstrate the instrument's capability due to their simplicity and widespread availability. These polystyrene particles are the standard particles used to calibrate the apertures that IZON manufactured. As the range of sizes supplied by IZON is limited to these five particles, custom synthesis of polystyrene and carboxylated polystyrene particles was also investigated. Polystyrene particles synthesized in this research ranged between 200 $\mathrm{nm}$ and $500 \mathrm{~nm}$. These particles were synthesized to give a better variety of diameters available to use in the calibration of apertures. Control over the size range is important especially in the nano-scale. However, further development of the technology in detecting metal particles may need calibration particles smaller than $50 \mathrm{~nm}$. Developing the methods to synthesize particles in the size region of $50 \mathrm{~nm}$ is essential to accurately calibrate the qNano to operate in this size regime.

\subsection{Measurements on qNano with submicron particles}

One of the first versions of IZON's aperture was made so that particles that have a diameter range between $0.1 \mu \mathrm{m}$ and $1 \mu$ can be detected. Initial measurements in this research will use particles that are within this diameter range. In this section, the polystyrene particles will be used mainly to map the aperture's geometric profile and as a learning tool to discover the capability of the instrument. 


\subsubsection{Aperture calibration}

As a standard procedure, each aperture manufactured by IZON went through a calibration procedure to ensure that the aperture is working as expected. This protocol was done to guarantee that the pore would open when stretched, the baseline current would be stable and that there would be no particle blockage. Most importantly, the calibration procedure was done to determine the lower and upper limit of the aperture's detection capability in terms of particle diameter. The particle diameter chosen to calibrate the aperture is dependent upon the dimension of the pore. The size of the pore was estimated by the recorded value of the force of the needle used to puncture the aperture. IZON gave this value on the data sheet provided with the apertures. Spherical polystyrene particles were used for calibration of the apertures, as the signals produced from spherical particles were much easier to recognise and analyse than non-spherical objects, such as a nanorods.

\subsubsection{Aperture stability and current profile}

While engaged in this research, it became clear that most of the apertures manufactured by IZON have a shelf life of a certain number of experiments before they began to produce inconsistent readings. For example, if a fresh Aperture $X$ is used today, the signals detected after $\mathrm{Y}$ amount of experiments will give a different reading than when it was first used. These inconsistencies are due to a number of factors, which include blocking of the nanopore by the particles becoming stuck in the pore, as well as the aperture stress-strain profile.

\subsection{Experimental methods}

Initially, the $800 \mathrm{~nm}$ standard particles from IZON were used to map stability and current profile of the aperture when stretched. The $800 \mathrm{~nm}$ particles were chosen, as they are the easiest to detect passing through the system. These particles were suspended in SEB in a dilution of 1:1000, as suggested by IZON, and a voltage of $0.3 \mathrm{~V}$ was run through the system.

The initial current reading with a stretch $(\Delta \mathrm{XY})$ of approximately $10 \mathrm{~mm}$ was taken as a reference with the $800 \mathrm{~nm}$ particles. Following that, the fluid cells were refreshed to 
avoid blockage of the pore: particles were taken out of the upper fluid cell, the bottom fluid cells were rinsed, and the aperture was flushed with double distilled water. Once the system had been refreshed and set up again, current readings as the pore was stretched were taken using the same $800 \mathrm{~nm}$ particles. These readings were taken at a $1 \mathrm{~mm}$ stretch interval for 1 minute.

\subsubsection{Results and Analysis}

Figure 3.2 shows a plot of baseline current against aperture stretch $(\triangle \mathrm{XY})$ measurement. As discussed in section 2.2, $\Delta \mathrm{XY}$ is the difference between the final and initial stretch of the aperture. Note that $\Delta \mathrm{XY}$ serve as an indicator on how much the pore is stretched and is not the actual size of the pore opening.

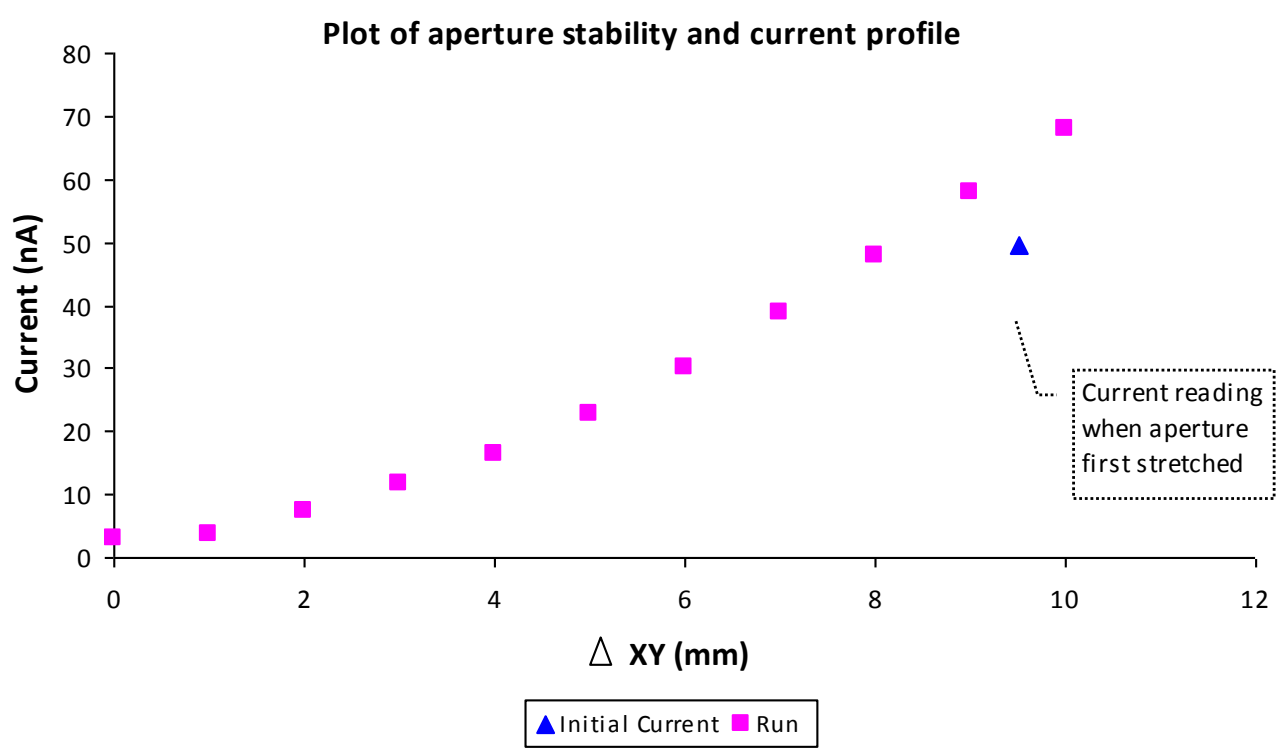

Figure 3.2: A typical current profile of apertures manufactured by IZON. A strong linear trend is not observed as expected due to softening of the membrane when it is stretched.

Figure 3.2 shows current readings at different pore stretch as $800 \mathrm{~nm}$ particles were passed through the aperture. The measurements were taken at a pore stretch between $0 \mathrm{~mm}$ and $10 \mathrm{~mm}$ with a $1 \mathrm{~mm}$ stretch interval. Figure 3.2 shows that for the $800 \mathrm{~nm}$ polystyrene particles, the initial current reading $\left(I_{0}\right)$ when the aperture was first stretched by $10 \mathrm{~mm}$ was approximately $50 \mathrm{nA}$. The pink data points in figure 3.2 
represent the measurements taken as the pore stretch was increased continuously without refreshing the fluid cells.

Comparing both $\mathrm{I}_{0}$ and the current measured at the same position after the system has been refreshed, data points in figure 3.2 shows that there was a difference in measured current even at the same stretching position. From figure 3.2, it can be seen that frequent stretching of the aperture for a period of time causes the instrument to give a different current reading. As the pore is stretched further than the maximum limit which allow Mullins effect to hold, the ability for the aperture to return to its original state decreases [80-85] as discussed in the chapter 2. This explains the higher current readings recorded as plotted in figure 3.2. The ability of the stretching mechanism to sensitively control the aperture stretch depends on how much the aperture has been used, as well as on the size of the pore itself. A well-used aperture will tend to show the 'creep' effect, which makes current readings either slowly increase or decrease depending on which direction it has been stretched. Additionally, apertures with a smaller pore will be damaged quicker as they are stretched further than apertures manufactured with a larger pore.

\subsection{Discussion}

There will be instances where unstable particle running was observed. This resulted in an erratic current as shown in figure 3.3.

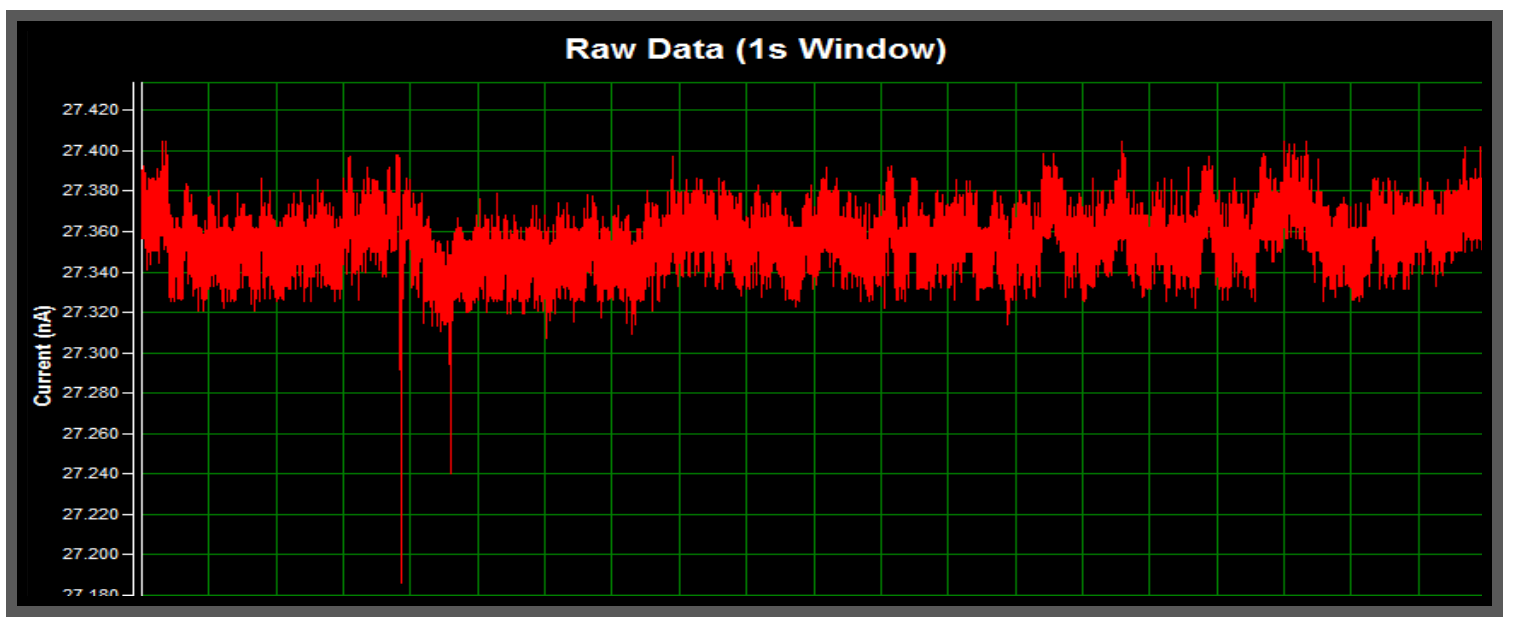

Figure 3.3: Screenshot of an example of an unstable measurement. The baseline current is erratic and a high level of background noise is observed. 
This current instability may have been due to pore geometry as well as particles that experience problems flowing through the pore. One hypothesis that explains the current instability is aperture softening and the conical shape of the nanopore, where the trans site of the nanopore may experience 'flapping'. This in turn will cause distortion of the current flow through the pore [89]. In addition to pore instability, particles tend to localise around the vicinity of the pore entrance. This is most prominent when the pore hole is in the order of the particle diameter, which would create further current instability.

\subsubsection{Optimising stretch for a single particle set}

The nature of the aperture means that blockade events are only registered within a window of stretch range. Therefore, it is crucial to determine where the optimum stretch lies to get the appropriate blockade events. As seen in the previous section, there are some instability issues when particles flow through the nanopore. Although the problem is minor and can be solved directly by changing the physical aspect of the experiment, another possible solution is to add a stability element in the system via electrostatic effects [25]. In this experiment, casein protein was added to the mixture to give stability to the particle translocation through the nanopore [22].

Casein is derived from mammalian milk and is part of phosphoproteins - proteins that are chemically bonded to substance that contains phosphoric acid. It consists of four different proteins which are the $\alpha_{s 1}, \alpha_{s 2}, \beta$, and $k$. Casein predominantly makes up $80 \%$ of the milk solution and $95 \%$ of casein in the milk exist in micelle form with diameters that can be as small as $25 \mathrm{~nm}$ or as large as $680 \mathrm{~nm}$. Casein is reported to have no typical denaturation temperature like other proteins and is described as 'rheomorphic'- a molecule that is flexible to adapt to the local environment of the solution i.e. it changes its structure according to the solution it's in [22].

Individual casein molecule will readily form a spherical micelle structure. This structure are made up of the hydrophobic layer in the insides of the micelle structure and the 'hairy' outer layer are made of macropeptide chains that are charged, hydrophilic, and acts as a stabilizer by steric and electrostatic effects which would prevent aggregation 
[22 - 25,50$]$. The $\mathrm{k}$-casein is an amphiphilic protein that has a hydrophobic region and a polar region. Due to this feature, $\mathrm{k}$-casein will spontaneously adsorb to the surface of polystyrene particles where the hydrophobic region attaches itself to the polystyrene particles and the hydrophilic region extends out to the solution [24].

\subsection{Experimental methods}

A high concentration of casein - here termed the Blocking Solution (BS) was used first to 'coat' the aperture with casein micelles. To make the blocking solution, $10 \mathrm{ml}$ of the standard buffer solution (SEB) is mixed with $0.5 \mathrm{~g}$ of casein powder. The casein powder (purchased from Aldrich) was used without further purification. Mixture of SEB and casein was vortexed and submerged in warm water to ensure homogeneity. Solution was then filtered with $0.22 \mu \mathrm{m}$ Millex GS MF-Milipore MCE Membrane. The blocking solution was then run through the aperture for at least 10 minutes to ensure that aperture was evenly coated.

The SEB was then laced with the blocking solution (here termed SEB-C) with a 1:1000 dilution by taking the blocking solution and adding it in the SEB. The mixture was then vortexed and filtered through $0.22 \mu \mathrm{m}$ filter. Prior to running SEB-C in the aperture, the system was washed with double distilled water for 2 to 3 times to remove excess blocking solution on the aperture.

Two sets of polystyrene particles were used to demonstrate the ability of the aperture to detect particle blockades. $400 \mathrm{~nm}$ and $780 \mathrm{~nm}$ standard polystyrene particles suspended in SEB-C were used in this experiment with a 1:1000 dilution. Both particle set were sonicated for 20 minutes before it was run through the pore. For the $400 \mathrm{~nm}$ particles, the pore was stretched to $\Delta X Y=19.46 \mathrm{~mm}$ and readings were taken as the pore is closed down to $\Delta \mathrm{XY}=17.69 \mathrm{~mm}$ in every current interval of approximately 1 $\mathrm{nA}$. A voltage bias of $0.2 \mathrm{~V}$ was used in this run and the current readings range from 47.3-43.37 nA.

Settings for the $780 \mathrm{~nm}$ particles includes stretching the aperture to a stretch of $\Delta \mathrm{XY}=$ $20 \mathrm{~mm}$ and decreased down to $\Delta \mathrm{XY}=15 \mathrm{~mm}$ at several different intervals to observe 
changes in quantity of blockade events. A voltage of $0.3 \mathrm{~V}$ was applied for this experiment. At each current interval, blockades readings were recorded for 1 minute and the blockade counts are plotted in figure 3.4.

\subsection{Results and Analysis}

In theory, it is expected that the rate should increase with pore size but as figure 3.4 shows, this is not so. The trend shows that after a certain point in the aperture stretch, blockade counts will decrease. This decrease is due to the smaller blockade magnitude as detected on the software as well as the increasing background noise of the instrument as the aperture is stretched.

\section{Distribution for $400 \mathrm{~nm}$ polystyrene}

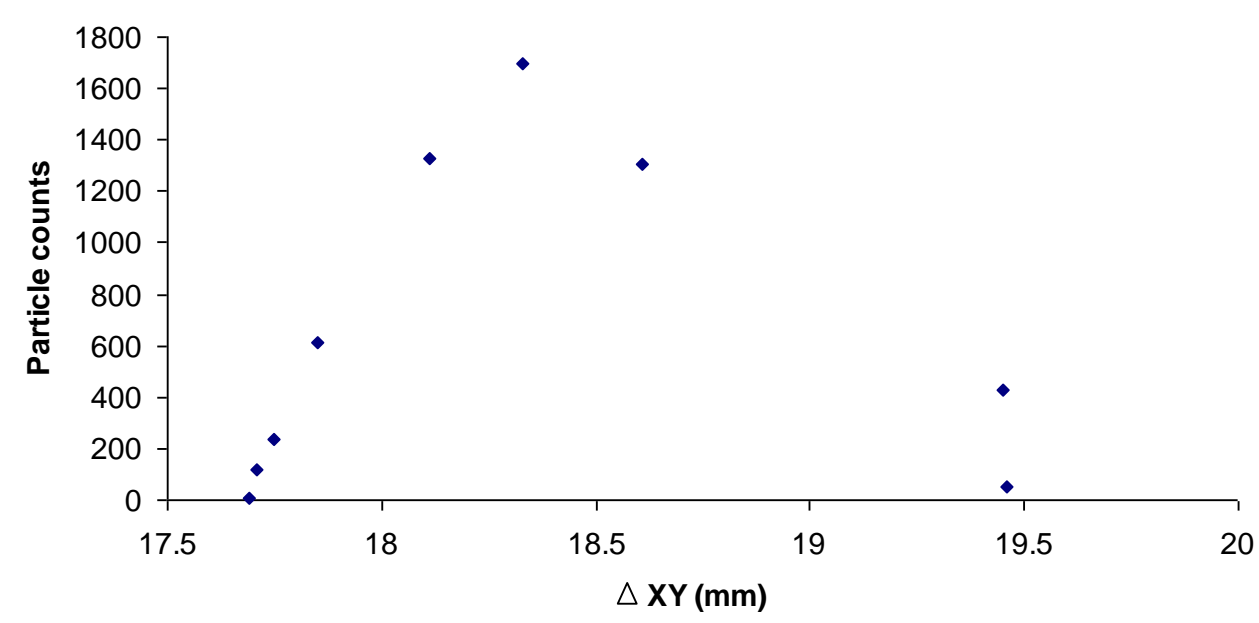

Figure 3.4: Blockade counts detected as a function of pore stretch. A concave plot was generated which gives an idea of a suitable working stretch range for each aperture.

Figure 3.4 shows the trend of blockades particles detected on this particular setting of the system. For each aperture, there is a range of stretch positions where particles translocation can be detected. Figure 3.4 shows that within each stretch range, there is a point where the maximum counts of particle blockades will be recorded. Whilst it is beneficial to have the maximum counts of particle blockades for quantitative studies, some of the recorded blockades could be due to both the particles as well as the background noise from the instrument hence making data interpretation challenging. Blockade counts registered on the software depends on the blockade magnitude 
threshold setting for each experiment. This setting is determined by utilising the noise data window that is available on the software.

The importance of finding the precise stretch for each measurement is demonstrated further with figure 3.5. Figure 3.5 shows measurements of $780 \mathrm{~nm}$ particles taken at four arbitrary stretches to observe the effect of particle blockade counts as a function of aperture stretch.

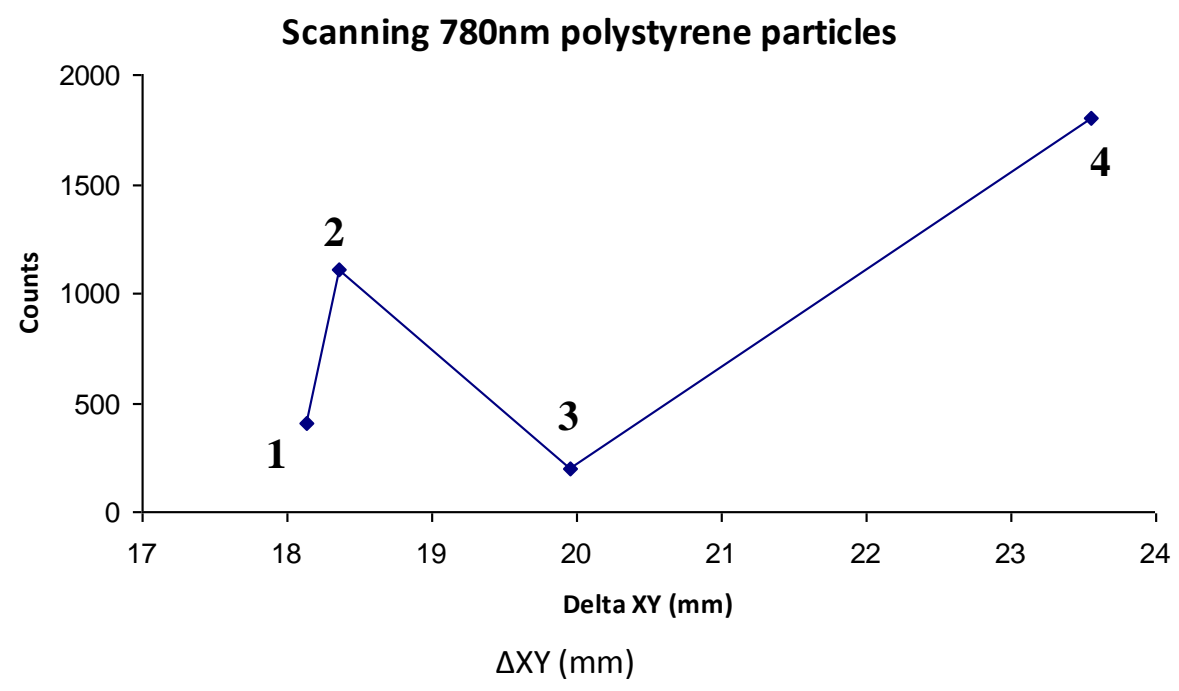

Figure 3.5: Blockade counts of $780 \mathrm{~nm}$ particles through the aperture at four different pore stretches. The plot is used to explain the importance of setting the measurement conditions and the choice of aperture stretch.

Blockade counts profile in figure 3.4 mirrors points $1-3$ in figure 3.5 . Point 4 in figure 3.5 shows blockade counts at a pore stretch that is much larger than the aperture's optimal stretch range. At point 4 , it can be seen that there is a surge of particle blockades recorded. This is not a true count of the particle blockades, as a larger pore stretch will give rise to a higher background noise, which is unfavourable. Without changing the 'Blockade Analysis' threshold settings on the software, the software will record the increased background noise on top of the particle translocation hence the increase in recorded translocation. Finding the suitable stretch range is definitely crucial for reason that even if the magnitude threshold settings are changed, the problem will still exist because the depth of particle blockade is relative to the pore opening. The magnitude of background noise increases while the magnitude of particle 
blockade's depth decreases with larger pore stretch. Therefore, this counteracting factor makes it important that a working aperture stretch is established.

\subsection{Discussion}

The number of particle blockades recorded by the instrument is relative to the pore size as seen in figure 3.4. A study by Willmott et al. [34] mentions an attempt to determine pore size readily from the current measurements. The study of blockade rate as a function of particle concentration shows deviation from the expected result by a factor of 2 and this assumed to be due to the geometric shape of the nanopore. These assumptions include calculation adjustments to accommodate a tapering cone geometry, large uncertainties in the radius of the pore on cis side, as well as the estimated linear and elastic response of the aperture to the stretching.

As explained earlier, the true counts of translocated particles depends on the magnitude threshold setting that was applied to each run in the experiment. One of the main factors that affect the counts of particles is the noise level. Few things can be attributed to the noise level, which includes noise from the main power source, $50 \mathrm{~Hz}$ interference noise generated from the electric field of other electrical appliances, as well as disturbance such as hand movements over the fluid cell. The latter will cause spikes to appear in the signal window as shown in figure 3.6. A metal cap is placed over the top fluid cell which acts as a Faraday shield to overcome this issue.

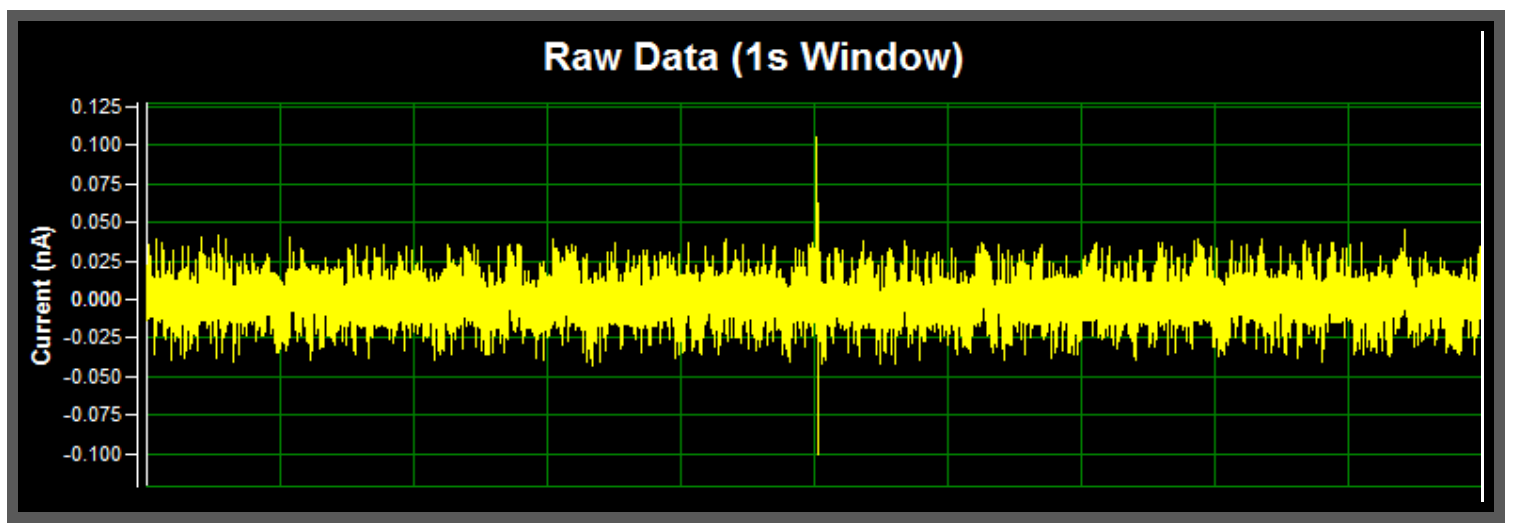

Figure 3.6: Noise spikes generated from hand movement over the fluid cell as seen in the "Normalised Signal" window. 
Another factor that might be affecting the translocation counts is the $10 \mathrm{kHz}$ bandwidth. This minimal bandwidth is required to discreetly resolve particles [13]. Signal profile given off by particles that requires a larger bandwidth will appear with shorter blockade depth on the live trace signal [13]. Therefore, particles that has a diameter of less than $100 \mathrm{~nm}$ requires signal bandwidth that is higher than $10 \mathrm{kHz}$ so that the resistive pulse given off by the particles can be accurately detected. Due to the minimum bandwidth available on this instrument, there might be instances where translocation of particles will not be recorded due to the short duration of translocation hence giving incorrect counts.

In conclusion, there is a range of stretch positions to work with in order to attain a true value of particles going through the nanopore. This range depends on the dispersity of the particle as well as the noise level. Figure 3.4 shows that the recorded counts are in a bell-shaped distribution.

\subsubsection{Bimodal distribution}

The ability to detect two different diameters of particles in a sample would open up a completely new market for this technology. Apart from analysing particles via Transmission Electron Microscopy (TEM) and Scanning Electron Microscopy (SEM) images, the only other available technology to analyse the distribution of particle diameters is using the Dynamic Light Scattering (DLS) method. However, the drawback of this technology is that it gives the average sizes of the particles in the sample instead of analysing the particles individually [10]. This puts IZON's technology in particle analysis at an advantage, as it is able to demonstrate the distribution of the particles in the sample and in this case is able to distinguish two different diameters of the same particle species. A recent publication by Roberts et al. [97] presents the work of characterizing DNA coated nanoparticles with IZON's qNano but more importantly, the publication discuss the study of bimodal and trimodal distribution of polystyrene particles in suspended in IZON's buffer solution as well. 


\subsubsection{Experimental methods}

The $400 \mathrm{~nm}$ and $780 \mathrm{~nm}$ standard polystyrene particles were suspended in SEB in a 1:1000 dilution. Voltage bias used in this run was set to $0.3 \mathrm{~V}$ and each sampled data was recorded individually for a period of 1 minute. In this experiment, the aperture was stretched to find $400 \mathrm{~nm}$ and $780 \mathrm{~nm}$ diameter particles independently. A constant measurement duration gives an estimate of the frequency of blockade events for both sets of particles. Once these values were obtained, appropriate ratio of each particle set was mixed and a bimodal distribution was obtained.

\subsubsection{Results and Analysis}

A high translocation frequency for both $400 \mathrm{~nm}$ and $780 \mathrm{~nm}$ particles ease the feasibility to construct the bimodal distribution. Measurement from the $400 \mathrm{~nm}$ particles saw counts of blockade events that are much higher than the $780 \mathrm{~nm}$ particles. The ratio of $400 \mathrm{~nm}$ to $780 \mathrm{~nm}$ particle mixture was determined by the count rate of each individual measurement that is given in Table 3.1.

\begin{tabular}{|l|l|l|l|}
\hline Diameter $(\mathrm{nm})$ & $\Delta \mathrm{XY}(\mathrm{mm})$ & Current $(\mathrm{nA})$ & $\begin{array}{l}\text { Frequency } \\
\text { (counts/min) }\end{array}$ \\
\hline 400 & 16.65 & 59.80 & 2484 \\
\hline 780 & 20.43 & 69.00 & 1821 \\
\hline $400: 780=5: 7$ & 20.47 & 72.30 & 2104 \\
\hline
\end{tabular}

Table 3.1: Data for blockades obtained for each set of particles and the mix

Table 3.1 shows the relevant data needed to build a bimodal distribution based on the individual particle set measurement. As it can be seen, $400 \mathrm{~nm}$ particles gave approximately $50 \%$ more counts than those detected for the $780 \mathrm{~nm}$ particles but the latter was done at a much higher stretch. This indicates that the $400 \mathrm{~nm}$ particles sample might be more concentrated than the $780 \mathrm{~nm}$ particles sample. Intuitively, to successfully generate a bimodal distribution, the sample mixture should contain a higher volume of the $780 \mathrm{~nm}$ particles to match the counts of the $400 \mathrm{~nm}$ particles. An appropriate mix of these two particles is crucial for the instrument to be able to 
distinguish the two diameters accurately as a disproportionate ratio could drown the signal from one diameter of the particles with another and present a false conclusion about the distribution.

Figure 3.7 shows both the independent particle distribution - the blue line represents distribution of the $400 \mathrm{~nm}$ and the pink line for $780 \mathrm{~nm}$, as well as the bimodal distribution - in yellow, that is obtained from this measurement.

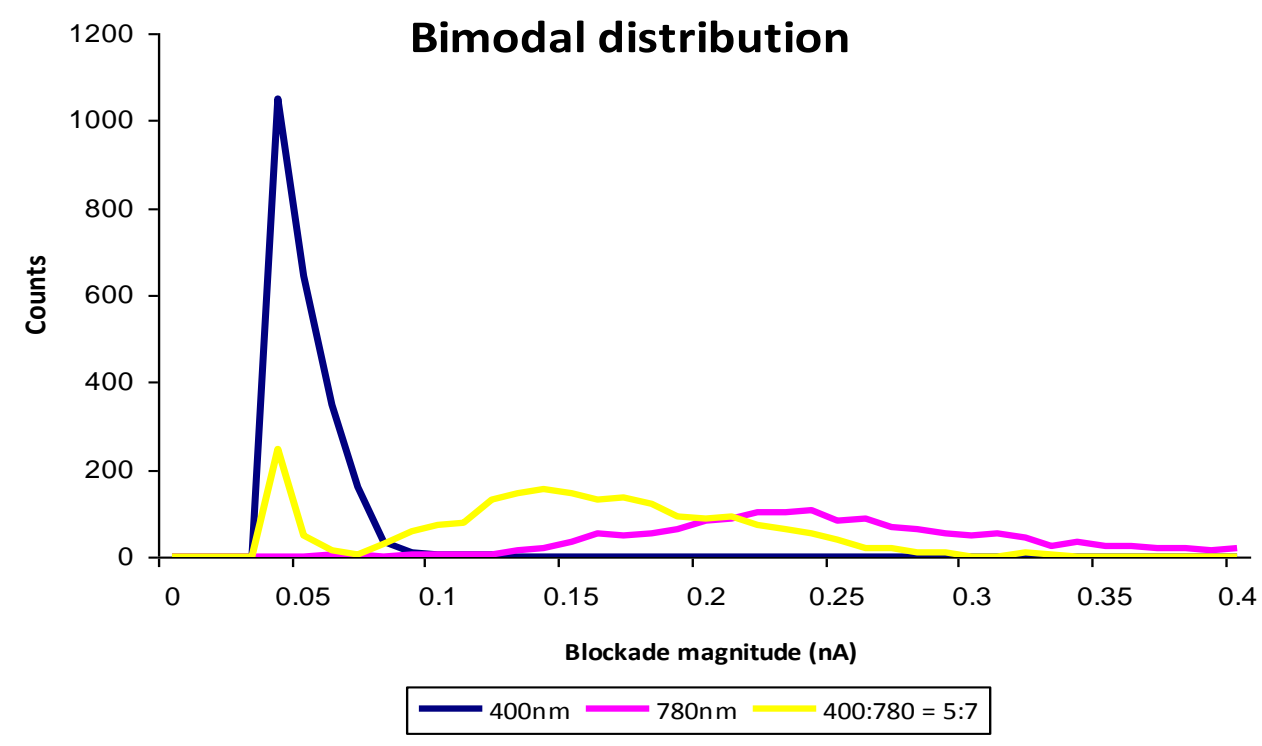

Figure 3.7: A plot of each particle set distribution (blue and pink) and the bimodal distribution (yellow) at a ratio of $400 \mathrm{~nm}: 780 \mathrm{~nm}=5: 7$.

Figure 3.7 also shows the instrument's ability to distinguish two polystyrene particles of $400 \mathrm{~nm}$ and $780 \mathrm{~nm}$ in diameter. The yellow plot in figure 3.7 represents the bimodal distribution, which appear to correspond to each of the individual particle line plots i.e. the blue and pink line. The plot in figure 3.7 shows that the $400 \mathrm{~nm}$ polystyrene particles have a tighter distribution as compared to distribution for the $780 \mathrm{~nm}$ particles where the distribution is much broader. A tighter distribution normally implies that the particles' distribution is monodispersed.

As reported in the work of Roberts et al. [97], the bimodal distribution plot should superimpose with the distribution plot from a single particle set. However, table 3.1 shows that the measurements were done at different stretches, which will cause a shift in the bimodal distribution with respect to the individual particles' distribution. 
The bimodal measurement was done at stretch of $\Delta X Y=20.47 \mathrm{~mm}$. A larger stretch means that the magnitude of particle blockades is smaller relative to the pore size as the particles displace a smaller amount of ionic density in the buffer solution as it passes through the pore. The distribution obtained also further stresses the importance of an appropriate ratio between the two sets of particles. As $780 \mathrm{~nm}$ particles are polydispersed, the peak of that distribution is approximately $20 \%$ of the peak for $400 \mathrm{~nm}$ particles. If the ratio of $400 \mathrm{~nm}$ to $780 \mathrm{~nm}$ is biased heavily towards the $400 \mathrm{~nm}$ particles, the peak of $780 \mathrm{~nm}$ will not be detected on analysis.

\subsubsection{Discussion}

IZON's apertures show that it can distinguish between two sets of different diameters of particles. This is an extremely useful feature and can be applied in areas where size selection is of importance. As demonstrated, the apertures are able to distinguish between particles with a diameter of $400 \mathrm{~nm}$ and $780 \mathrm{~nm}$. However, problems can arise when the size difference between the two particles are minimal for example, to selectively distinguish particles that is $300 \mathrm{~nm}$ or $350 \mathrm{~nm}$. This setback is dependent on how the apertures are fabricated i.e. the material and needle used to puncture the hole in the aperture. Further development of these apertures could add on to the advantages of this research instrument. In recent times, IZON has developed a new generation of the nanopore that is able to resolve particles that has a size difference of less than $100 \mathrm{~nm}$. This is validated with the latest publication by Roberts et al. [97], which showed that $80 \mathrm{~nm}$ and $50 \mathrm{~nm}$ particles suspended in the buffer solution could be distinguished.

Another problem with size selecting using this technology is that samples with a high polydispersity would be harder to separate. Sample that is polydispersed would have a large range of stretch positions. Therefore, if both of the samples used are polydispersed, there is a high chance that the two stretch ranges for each particle set will overlap each other. In this case, the suitable stretch range would be a lot harder to determine and size selection for particles will not be accurate. 


\subsubsection{Effect of surface functionality of polystyrene particles}

IZON supplies carboxylated polystyrene particles that have a diameter of $70 \mathrm{~nm}, 100$ $\mathrm{nm}, 200 \mathrm{~nm}, 400 \mathrm{~nm}$, and $780 \mathrm{~nm}$ in their standard starter kit to serve as a reference set. As there is only limited range of particle diameters supplied from IZON, synthesizing our own carboxylated polystyrene particles in a diameter range that is not supplied by IZON will give a sense of control over the diameter of calibration particles to be used with the instrument. This is crucial as control over the diameter of calibration particles would be of important use in the later part of the research where metal and other noble particles are involved. Here, both types of polystyrene particles will be synthesized - functionalised and un-functionalised. The aim of this measurement is to determine if there is a difference in signal blockades if a polystyrene particle is used compared to a carboxylated polystyrene particle.

\subsubsection{Synthesis of polystyrene and carboxylated polystyrene particles}

Polystyrene particles were synthesized via emulsifier-free emulsion polymerisation method $[18,19,20]$. This method was undertaken as it gives particles that are in the range of sub micrometer. Particles synthesized with this method was mainly characterised by electron microscopy.

\subsection{Method}

\section{Polystyrene particles}

Method developed by Yan and Chen [21] to synthesize polystyrene particles uses styrene (purchased from Aldrich) and distilled water bubbled with nitrogen gas for 15 minutes under vigorous stirring in a three-neck round bottom flask. The solution was heated up to $70^{\circ} \mathrm{C}$ for 20 minutes until thermal equilibrium was reached. Once the temperature had stabilized, benzoyl peroxide, which acts as the radical initiator, was added to the heated solution. The solution was left to polymerise at $70^{\circ} \mathrm{C}$ for a set of time. After approximately 4 hours of reacting, the solution changes from colourless to a milky white solution. Solution was then cooled to room temperature and filtered with $0.3 \mu$ membrane. 
Four sets of polystyrene particles were made. These particles differ from each other by the amount of starting reagent used as well as the reaction time. All other parameters were held constant. A summary of the successful reaction conditions is given in Table 3.2.

\begin{tabular}{|l|l|l|l|l|}
\hline Experiment & $\begin{array}{l}\text { Styrene } \\
(\mathrm{ml})\end{array}$ & $\begin{array}{l}\text { Distilled } \\
\text { water }(\mathrm{ml})\end{array}$ & $\begin{array}{l}\text { Benzoyl } \\
\text { Peroxide }(\mathrm{g})\end{array}$ & $\begin{array}{l}\text { Time } \\
\text { (hours) }\end{array}$ \\
\cline { 1 - 3 } PS01 & 1.2 & 18.8 & 0.017 & 4 \\
\cline { 1 - 1 } PS02 & & & & 12 \\
\cline { 1 - 1 } PS03 & 2.0 & 18.0 & 0.017 & 9 \\
\cline { 1 - 1 } \cline { 1 - 1 } & & & & 12 \\
\hline
\end{tabular}

Table 3.2: Reaction condition used to synthesise polystyrene spheres.

\section{Carboxylated polystyrene particles}

Functionalised polystyrene particles here were synthesized based on the method developed by Wang and Pan $[18,19,20]$. A mixture of $20 \mathrm{ml}$ of distilled water and 2.2 $\mathrm{ml}$ of styrene (purchased from Aldrich) were mixed together in a three-necked round bottom flask. This mixture was heated up and magnetically stirred with nitrogen gas bubbled through the mixture for 10 minutes. Next, $0.22 \mathrm{~g}$ of methyl acrylic acid (MAA) (Aldrich) was then added and temperature of the solution was increased to $70^{\circ} \mathrm{C}$, which stabilizes after 40 minutes. Then $0.01073 \mathrm{~g}$ of benzoyl peroxide (Aldrich) was then added to the round bottom flask and solution was left to react for another 7 hours. All reagents were used without further purification.

All of the particles were purified by centrifuge at 5000rpm for 15 minutes, washed several times with double distilled water, and stored at room temperature.

\subsection{Risk assessment}

Listed in Table 3.3 is the risk assessment of the chemicals used to synthesize the polystyrene particles [91]. 


\begin{tabular}{|c|c|c|}
\hline Chemicals & Risk & Danger \\
\hline \multirow[t]{2}{*}{ Styrene } & \multirow[t]{2}{*}{$\begin{array}{l}\text { Very } \\
\text { hazardous }\end{array}$} & $\begin{array}{l}\text { Irritant to the eye. Inflammation of the eye is characterized } \\
\text { by redness, watering, and itching. }\end{array}$ \\
\hline & & Hazardous to skin contact, ingestion, and inhalation. \\
\hline \multirow[t]{6}{*}{$\begin{array}{l}\text { Benzoyl } \\
\text { Peroxide }\end{array}$} & \multirow[t]{6}{*}{$\begin{array}{l}\text { Extremely } \\
\text { hazardous }\end{array}$} & $\begin{array}{l}\text { Very hazardous in case of skin contact (irritant), of eye } \\
\text { contact (irritant), of inhalation. }\end{array}$ \\
\hline & & Prolonged exposure may result in skin burns and ulcerations. \\
\hline & & Over-exposure by inhalation may cause respiratory irritation. \\
\hline & & $\begin{array}{l}\text { Inflammation of the eye is characterized by redness, } \\
\text { watering, and itching. }\end{array}$ \\
\hline & & $\begin{array}{l}\text { Skin inflammation is characterized by itching, scaling, } \\
\text { reddening, or, occasionally, blistering. }\end{array}$ \\
\hline & & Contact with eyes, respiratory tract or skin will cause burns. \\
\hline \multirow{2}{*}{$\begin{array}{l}\text { Methyl } \\
\text { acrylic } \\
\text { acid }\end{array}$} & \multirow[t]{2}{*}{ Corrosive } & Harmful by skin contact \\
\hline & & $\begin{array}{l}\text { Typical Occupational Exposure Level (OEL) is } 20 \mathrm{ppm} \text {. May act } \\
\text { as a mutagen. }\end{array}$ \\
\hline
\end{tabular}

\section{Table 3.3: Risk Evaluation}

\subsection{Results and Analysis}

The synthesized particles were characterised using the Scanning Electron Microscope (SEM) for images and for size distribution analysis. Approximately 200-300 particles were manually sized using the scale bar attached to the images taken using JEOL's SemAfore; a digital image analysis software on top of cross checking with Zetasizer. From the analysis, histograms are built to see the size distributions of the sample.

\section{Polystyrene particles}

\section{PSO1 and PSO2}

As shown in table 3.2, the amounts of precursor used to make these particles were kept constant at $1.2 \mathrm{ml}$ of styrene and $18.8 \mathrm{ml}$ of deionised water. The only variable was the reaction duration where PS01 was left to react for 4 hours and PSO2 was left 
to react for a period of 12 hours. Figure 3.8 shows the image of the synthesized polystyrene particles at different reaction conditions.

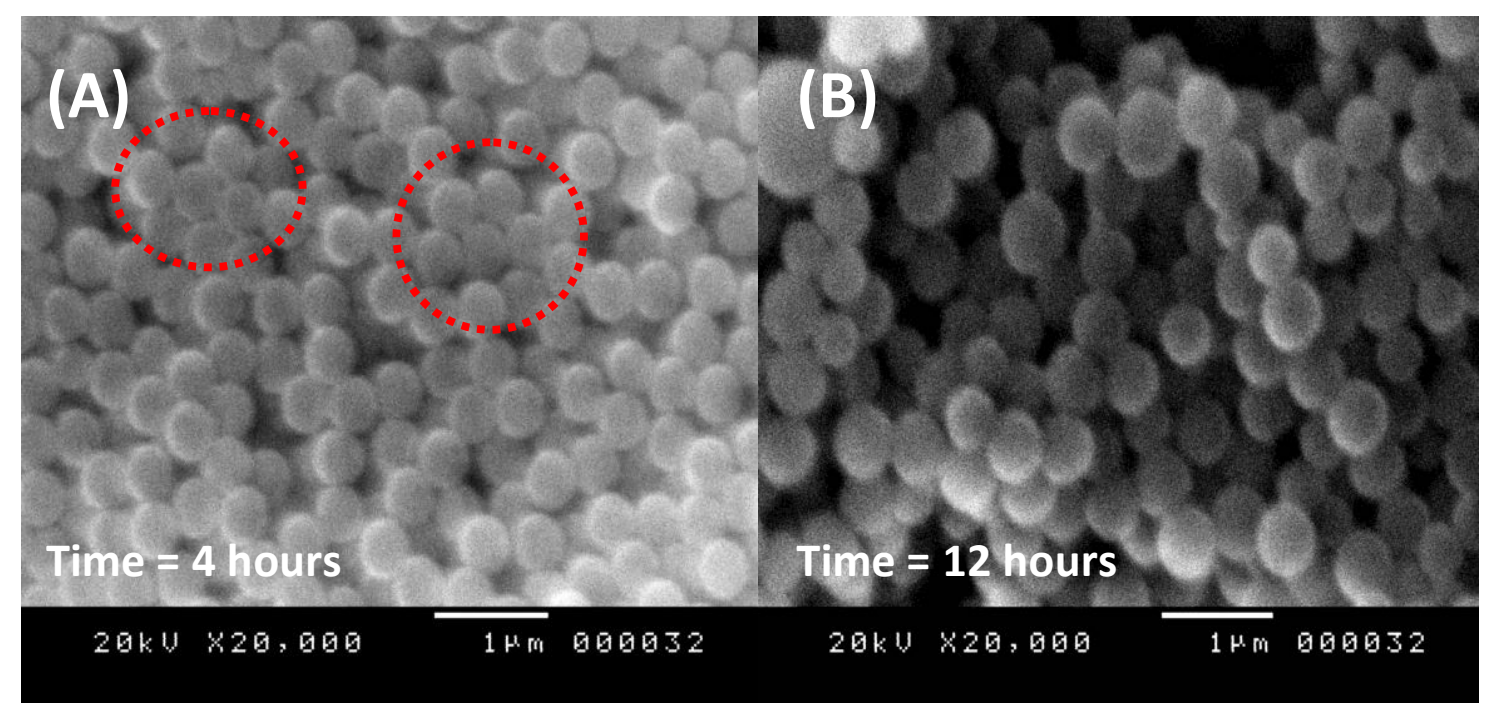

Figure 3.8: SEM images of polystyrene samples. (A) PSO1 sample has a diameter of approximately $420 \mathrm{~nm}$ (B) PSO2 with a diameter of approximately $500 \mathrm{~nm}$.

Images form sample PSO1 shows particles with some feature of a monodisperse sample by the presence of a hexagonally close-packed structure (as shown in the red bubble). Sample PSO2 however shows particle aggregation and the absence of a closepacking structure in the image implies that the sample has a non-uniform particle size distribution. The images in figure 3.8 show that the duration of reaction plays a role in the quality of the particles [21]. However, the cause was not investigated further in this thesis. A histogram plot is constructed to show the distribution of sample PSO1. 


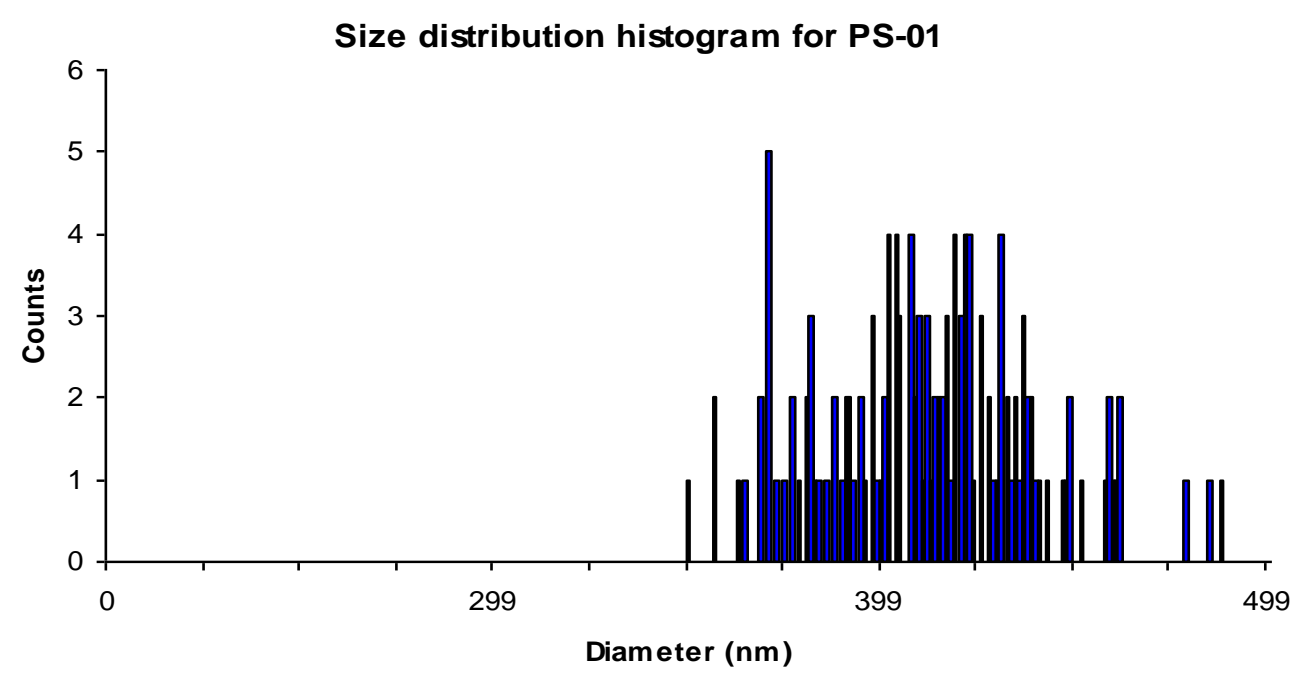

Figure 3.9: Size distribution for PSO1 as analysed using SemAfore. The distribution gives a mean value of $420 \mathrm{~nm} \pm 75 \mathrm{~nm}$.

The histogram in figure 3.9 is used to analyse the particle size distribution in sample PS01. The figure shows that sample has a mean diameter of approximately $420 \mathrm{~nm} \pm$ $75 \mathrm{~nm}$. Around 200 particles were used to build the histogram. As it can be seen, the distribution is very wide i.e. having the smallest analysed particle around the $350 \mathrm{~nm}$ mark and the largest particle almost reaching $500 \mathrm{~nm}$ mark. There is no histogram built for sample PSO2 as samples are too aggregated to accurately measure each individual particle. Nonetheless, the sample were characterised via the dynamic light scattering technique (Zetasizer). This technique gives the diameter of sample PSO2 to be approximately $500 \mathrm{~nm}$. The instrument however did not give the percentage of standard deviation.

\section{PSO3 and PSO4}

In search of an acceptable size range (and quality) of polystyrene particles, two other sets of polystyrene particles were synthesized using different amount of precursor. Higher concentrations of styrene ( $2 \mathrm{ml}$ in $18 \mathrm{ml}$ of deionised water) as well as a varying reaction time were used, as tabulated in Table 3.2. These particles are labelled PSO3 and PSO4 and images taken are given in figure $3.10(\mathrm{~A})$ and (B). 


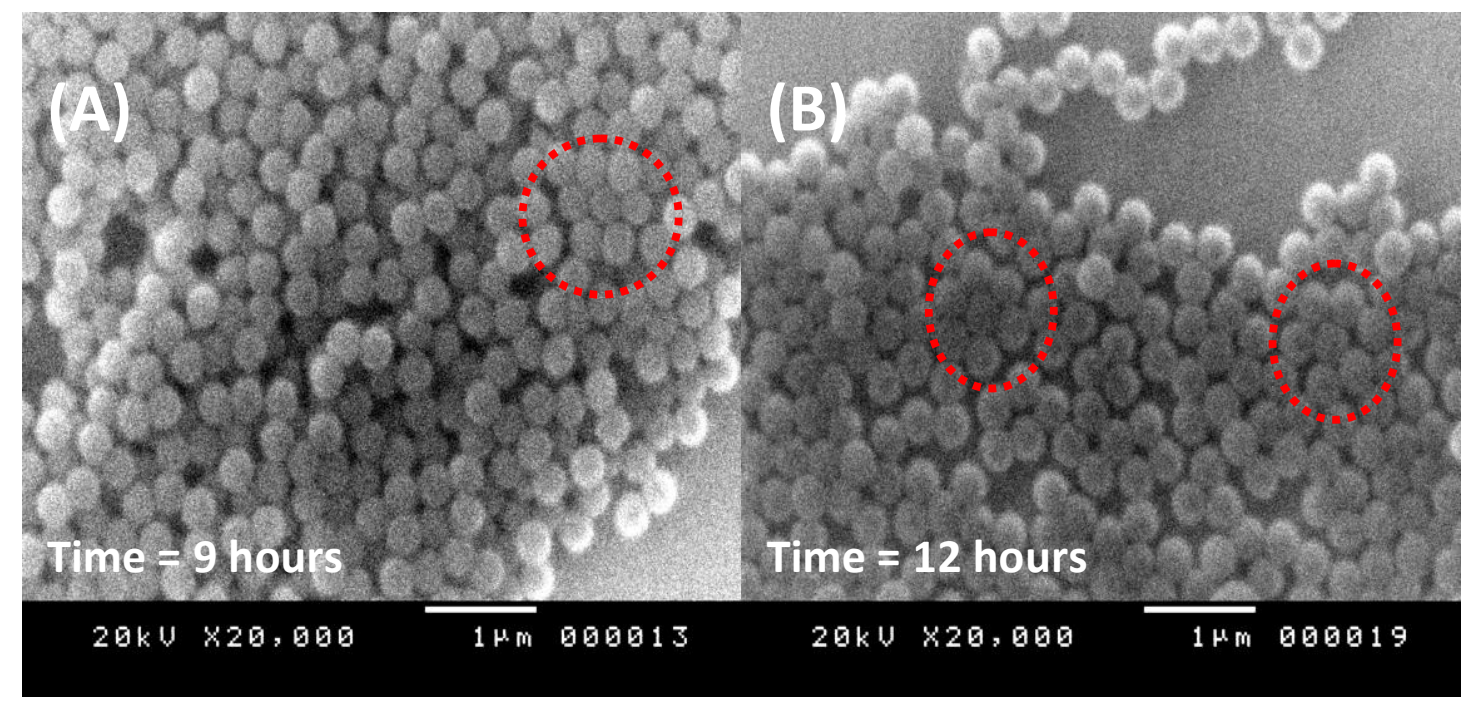

Figure 3.10: SEM images of polystyrene samples. (A) PSO3 sample is approximately $350 \mathrm{~nm}$ in size. (B) PSO4 sample is approximately $310 \mathrm{~nm}$ in size.

Figures 3.10 (A) and (B) both show the image of PSO3 and PSO4 respectively. By analysing these images as given in figure 3.10 , there is no distinctive difference that can be noticed between these two sets of particles. Both PSO3 and PSO4 show a uniform sized sample with the potential to have a monodisperse distribution by the presence of the hexagonal close packed structure (in red bubble). A histogram distribution in figure 3.11 was constructed to make a more concrete summary of its distribution.

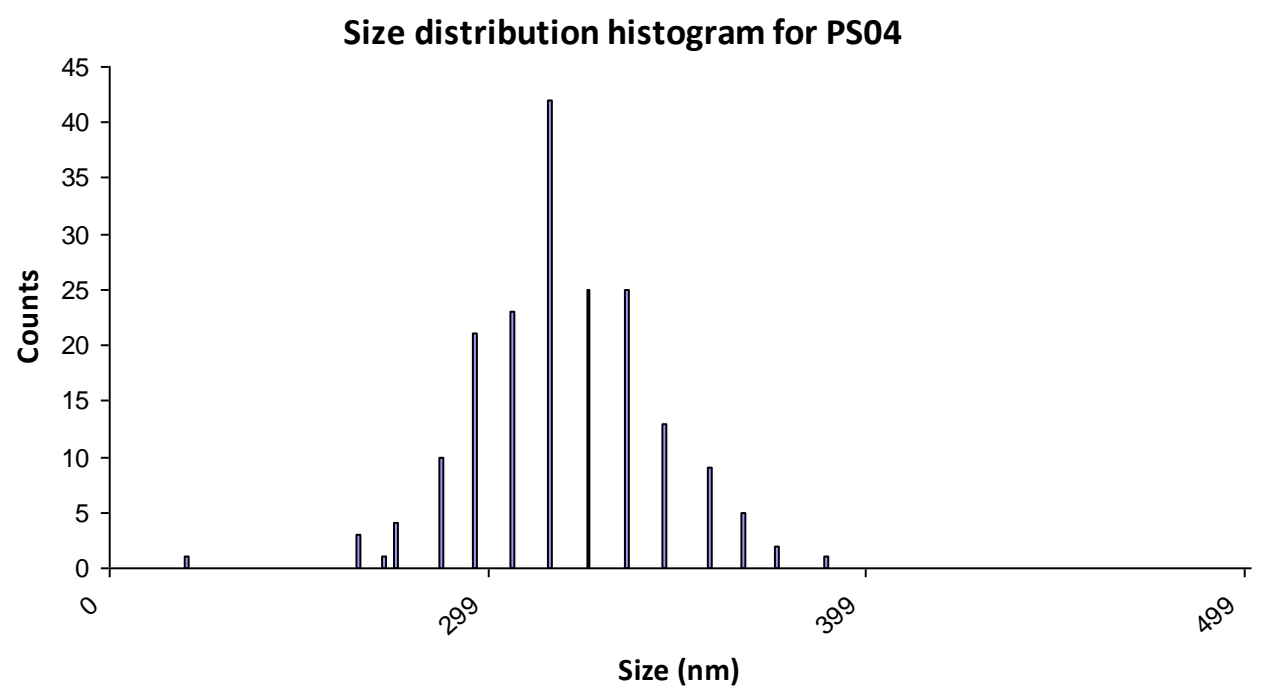

Figure 3.11: Size distribution of polystyrene particles PS04. An average of 200 particles were analysed to make up the histogram. The distribution gives a mean value of 310 $n m \pm 63 n m$. 
Figure 3.11 shows that sample PSO4 has a distribution with a mean diameter of $310 \mathrm{~nm}$ $\pm 63 \mathrm{~nm}$. Results from this distribution imply that the sample has a standard deviation of $20 \%$ from its average diameter. Characterising sample PSO3 with the Zetasizer gives a mean diameter of $350 \mathrm{~nm}$ with no standard deviation value given although it is assumed to be polydispersed judging by the image in figure 3.10 .

\section{Carboxylated polystyrene particles}

The carboxylated polystyrene particles was characterised using the Scanning Electron Microscope (SEM) for image and size distribution analysis. These particles were manually sized using the scale bar attached to the images taken using JEOL's SemAfore. Figures 3.12 and 3.13 both show the results obtained from SEM.

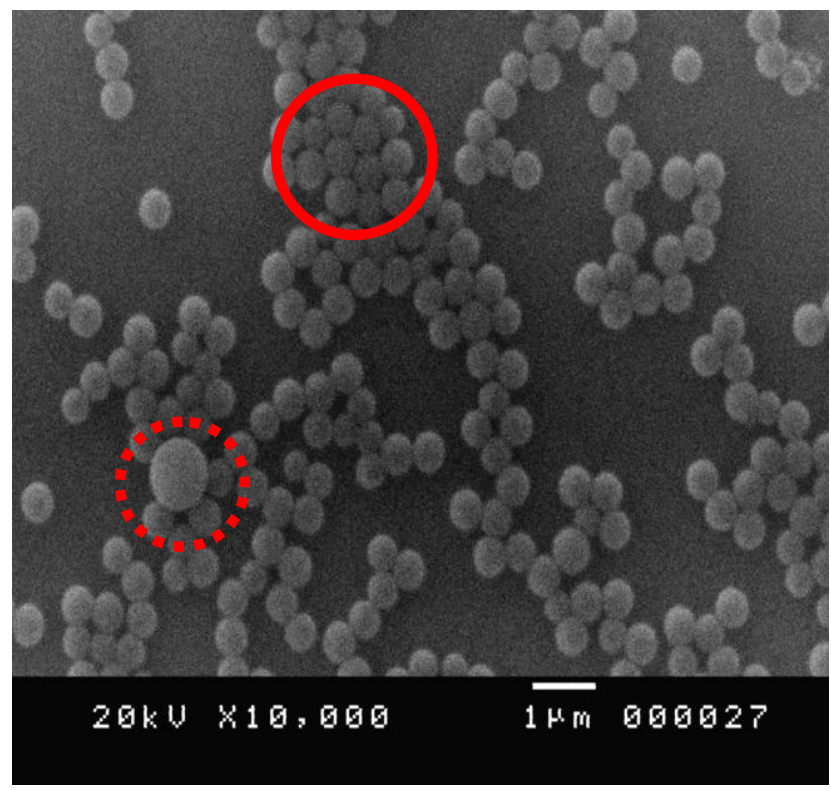

Figure 3.12: SEM image of the synthesized carboxylated polystyrene particles samples at x10, 000 magnifications. Visual analysis of the image seems to show that the polystyrene sample is monodispersed.

Figure 3.12 above shows an image of CTPS01 synthesized using a method developed by Wang et al. [18 -20]. From the image, it can be seen that the particles surface are much 'cleaner' as compared to particles synthesized in the PS series i.e. PS01, 02, 03, and 04 . CTPSO1 particles are distinctively separated in comparison to the normal polystyrene particles. Figure 3.12 shows that the sample has a broad dispersity and this observation is confirmed by the histogram plotted in figure 3.13. 


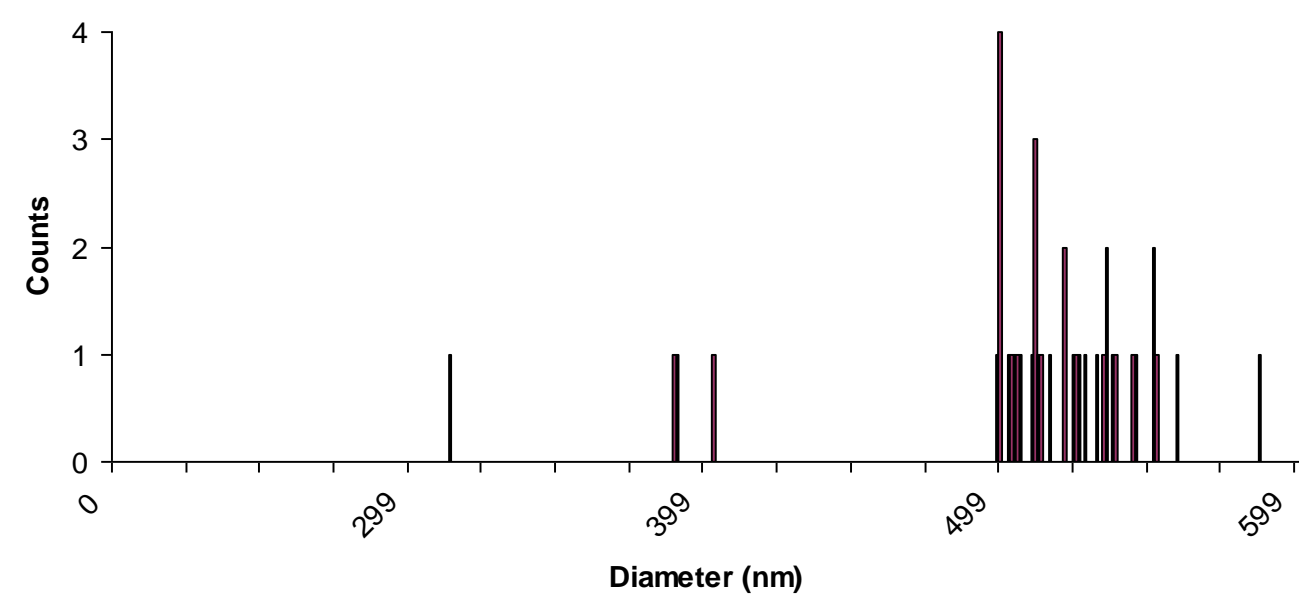

Figure 3.13: Size distribution of carboxylated polystyrene particles gives an average of $520 \mathrm{~nm}$ with a standard deviation of approximately $10 \%$.

Figure 3.13 shows the particle size distribution of CTPSO1 as analysed using SemAfore. As expected, the distribution of the particles is broad with an average diameter of 520 $\mathrm{nm}$ with a $10 \%$ standard deviation to the particle size.

\subsection{Synthesis discussion}

\section{Polystyrene particles}

The parameter that was changed in all the synthesized particles is the concentration of styrene used as well as the reaction time. For all reactions, the amount of radical initiator used remained constant at $0.017 \mathrm{~g}$. This is crucial in determining the diameter of the particles formed. A higher concentration of styrene means that there are more styrene monomers available for the radical initiator to start the polymerisation process. This explains the smaller particle diameter that was generated in samples PSO3 and PSO4 as compared to samples PSO1 and PSO2.

As demonstrated in both figures 3.8 and 3.10, the reaction time especially plays an important role in determining the size distribution of the particles. Yan and Chen [21] discuss that a polymerization time of 4 hours would give a non-uniform dispersity (as seen in sample PS02). Their study also shows that a reaction time that is less than 8 hours would give a distribution that has a standard deviation that is more than $5 \%$ of 
their mean value. In contrast, a reaction time that is more or equal than 12 hours produce particles that have a $5 \%$ standard deviation to the average diameter. Essentially, the dispersity of the sample plays a minimal factor in determining which sample is to be used. However, having a monodisperse sample would make an easier identification of the blockade signal. Hence, with the samples available, sample PSO4 will be dominantly used in subsequent measurement on the qNano.

\section{Carboxylated particles}

Wang and Pan [20] discuss that copolymerisation of styrene and methyl acrylic acid (MAA) produce high concentration of carboxylated terminals on the particle surface. This is due to the change of polymerisation locus after a period of time into the synthesis. The styrene polymerises via a homogeneous nucleation during the initial part of the synthesis. Addition of MAA to the solution is reported to have a comparatively low percentage of diffusing into the styrene particles and resides mostly on the outer layer of the particles [20].

There is an increase in particles diameter as polymerisation of styrene continues and declining amount of styrene monomers available is the start of the next stage of the copolymerisation. As stated, the MAA monomers are very water-soluble. Therefore, it is difficult to ensure that the MAA monomer bonds with the polystyrene particles. However, the MAA monomer bonds either by forming oligomer radical with styrene monomers or via bonding with diffused MAA monomers in the particles during the first stage of polymerisation. The latter forms a layer on the 'outside' of the particles, which then induce MAA polymerisation to the outer layer. The swelling of this outer layer increase the diameter of the particles as well as giving the particles its hydrophilicity and its steric stabilization [20].

The distribution of carboxylated polystyrene particles sample is unclear because image given in figure 3.12 portrays a monodisperse distribution (as shown in solid red line) yet there is also presence of a much larger particle as marked by the dashed red line. Nevertheless, on the whole this sample has a good distribution of diameter and 
together with IZON's supplied particles, gives an array of particle diameter to work with.

\subsection{Synthesis conclusion}

Of all the particles synthesized, particles PS02, PS04, and CTPS01 will be used because of the average particle diameter. PSO4 is run against IZON's standard $800 \mathrm{~nm}$ carboxylated particles and PS02 against CTPS01. Measurements on these particles are detailed in the next section.

\subsubsection{Experimental method}

IZON's $800 \mathrm{~nm}$ standard polystyrene particles, PS02, PS04, and CTPS01 particles were used in this experiment. PSO4 and $800 \mathrm{~nm}$ particles were suspended in SEB in a 1:1000 dilution while PSO2 and CTPS01 were suspended in 1:500 dilutions. All solutions were sonicated for 20 minutes prior to use. Both of these measurements were found to be running in a good condition on a voltage bias of $0.2 \mathrm{~V}$.

\subsubsection{Results and Analysis}

The aim for this section of experiment is to see if there is any difference between unfunctionalised and functionalised polystyrene particles suspended in SEB. As the key difference lies on the surface properties of the particles, the duration of translocation is studied. Figure 3.14 and 3.15 gives the overall distribution of magnitude and duration of the particles going through the pore. Each point corresponds to a particle detected as they go through the nanopore. 


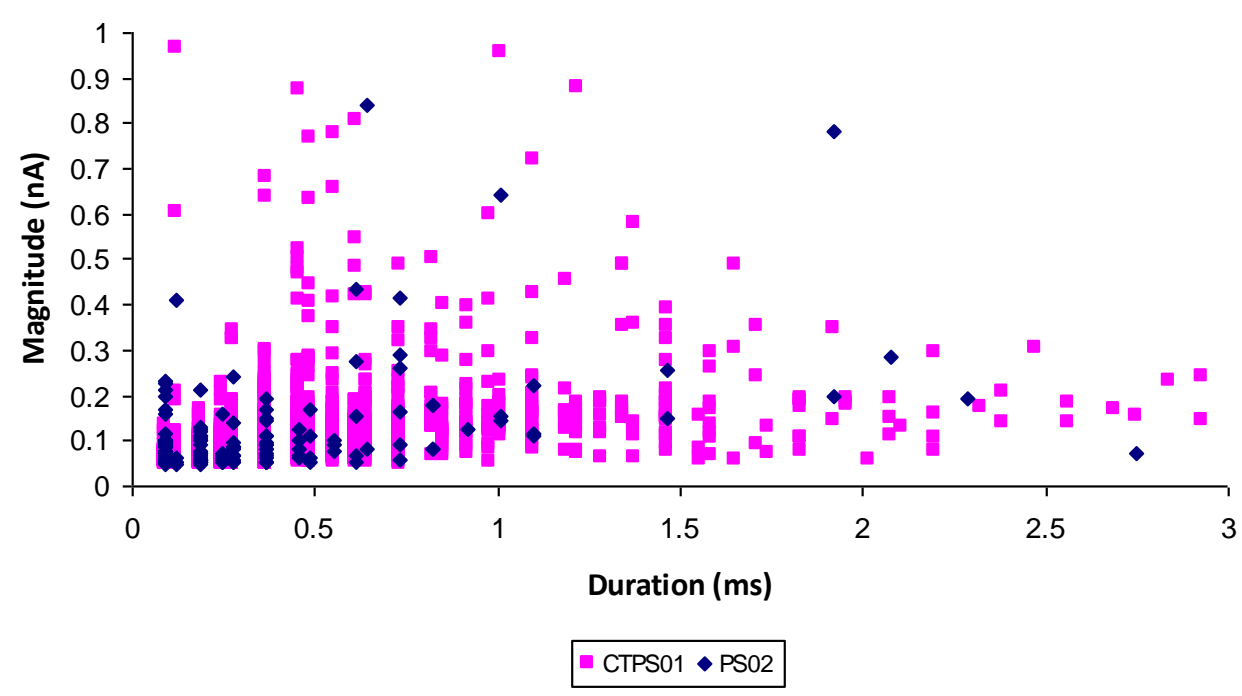

Figure 3.14: A distribution plot of CTPSO1 and PSO2 as they translocate through the nanopore. This serves as a quick tool to illustrate the dispersity of the particle sample.

Figure 3.14 shows the particle blockades distribution of CTPSO1 with a mean diameter of $520 \mathrm{~nm}$ and PSO2, which has a mean diameter of $500 \mathrm{~nm}$. As expected, figure 3.14 shows that both of PSO2 and CTPS01 particles concentrate in the corner of the two axes i.e. at less than $2 \mathrm{~ms}$ with blockade magnitude of less than $0.4 \mathrm{nA}$. It is important to note that these two sets of particles have an average diameter difference that is comparatively small, approximately $20 \mathrm{~nm}$. As is shown in section 3.2.2, it is difficult to distinguish between two particle diameters especially if the difference between the two is small. Therefore, as the two sets of particles are run beside one another the signals obtained confirm that the two particle set sizes approximate each other.

The difference between IZON's $800 \mathrm{~nm}$ particles against PSO4 was also studied and the distribution is given in figure 3.15. 


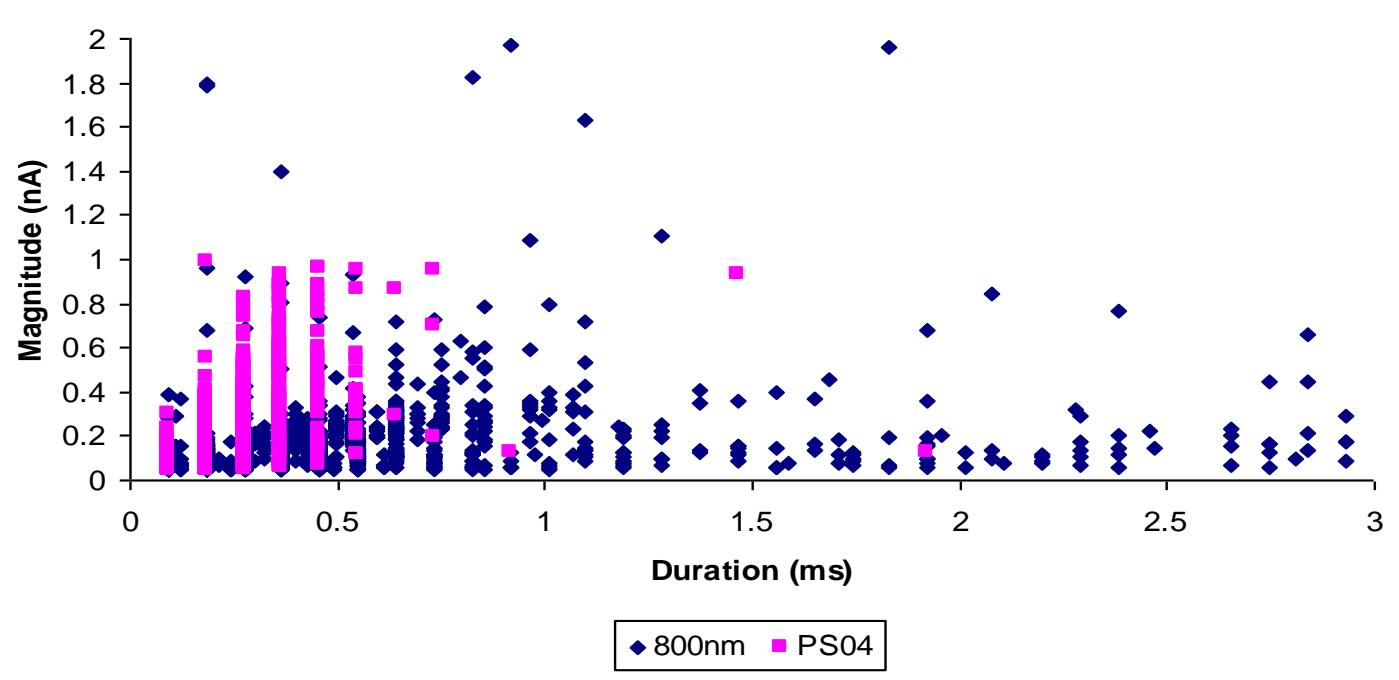

Figure 3.15: A plot of magnitude as a function of duration shows the distribution of PSO4 and $800 \mathrm{~nm}$ IZON particles as they translocate through the nanopore.

Figure 3.15 shows the particle blockades distribution of PSO4 with a diameter of 310 $\mathrm{nm} \pm 63 \mathrm{~nm}$ and IZON's $800 \mathrm{~nm}$ particles. In this measurement, 3500 blockade events were detected in the space of 5 minutes. Figure 3.15 also shows that the scatter points for PSO4 illustrate a much wider size dispersity as compared to the $800 \mathrm{~nm}$ particles. This is given by the broad blockade magnitude range i.e. 0-1 $\mathrm{nA}$ as compared to approximately $0-0.5 \mathrm{nA}$ for the $800 \mathrm{~nm}$. This is explained by the dispersity of both sets of particles. IZON's $800 \mathrm{~nm}$ particles may have a more monodisperse particles as compared to PSO4, which has a standard deviation of $20 \%$. From the duration of translocation point of view, it can be seen that PSO4 goes through the nanopore at a narrow distribution time i.e. between 0-0.6 ms while $800 \mathrm{~nm}$ polystyrene particles go through the pore at a wider distribution of 0-1 ms.

Translating the $\mathrm{Y}$-axis of figures 3.14 and 3.15 into a histogram produces figure 3.16 and 3.17 respectively. These plots will give a clearer picture of the blockade distribution. Figure 3.16 shows the distribution of blockade magnitudes for PSO2 and CTPS01. 


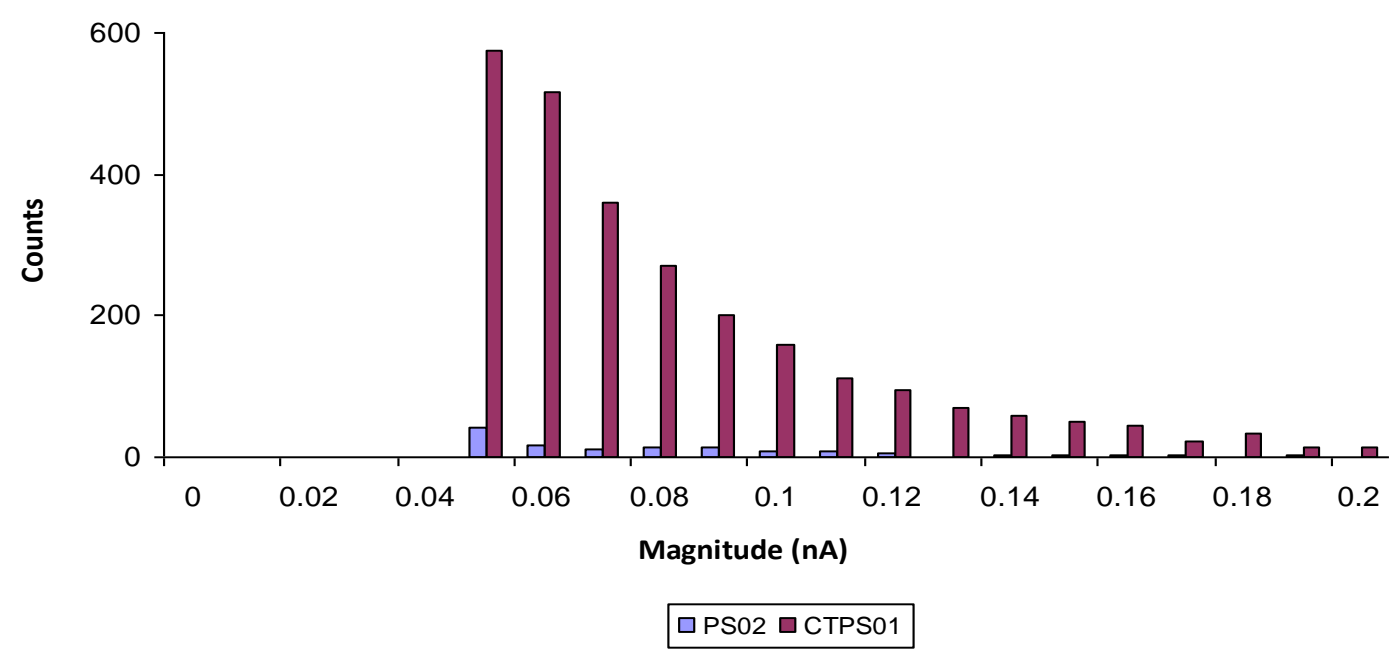

Figure 3.16: Histogram plot showing the distribution of blockade magnitudes for PSO2 and CTPSO1 particles suspended in SEB. The counts for carboxylated particles exceed the counts for polystyrene particles possibly due to the difference in the raw concentration of particles.

Signals from CTPS01 are expected to approximate signals from PS02 such as shown in figure 3.16. The histogram peaks at a value of $0.05 \mathrm{nA}$ for both set of particles. This shows that the instrument is yet to differentiate between particles of $500 \mathrm{~nm}$ and 520 $\mathrm{nm}$ in diameter. Figure 3.16 also shows that the instrument detects a broad distribution in figure 3.13 for sample CTPSO1 that tallies with the result analysed via SemAfore.

Magnitudes for PSO4 and $800 \mathrm{~nm}$ particle blockades were also analysed and the distribution is given in figure 3.17. 


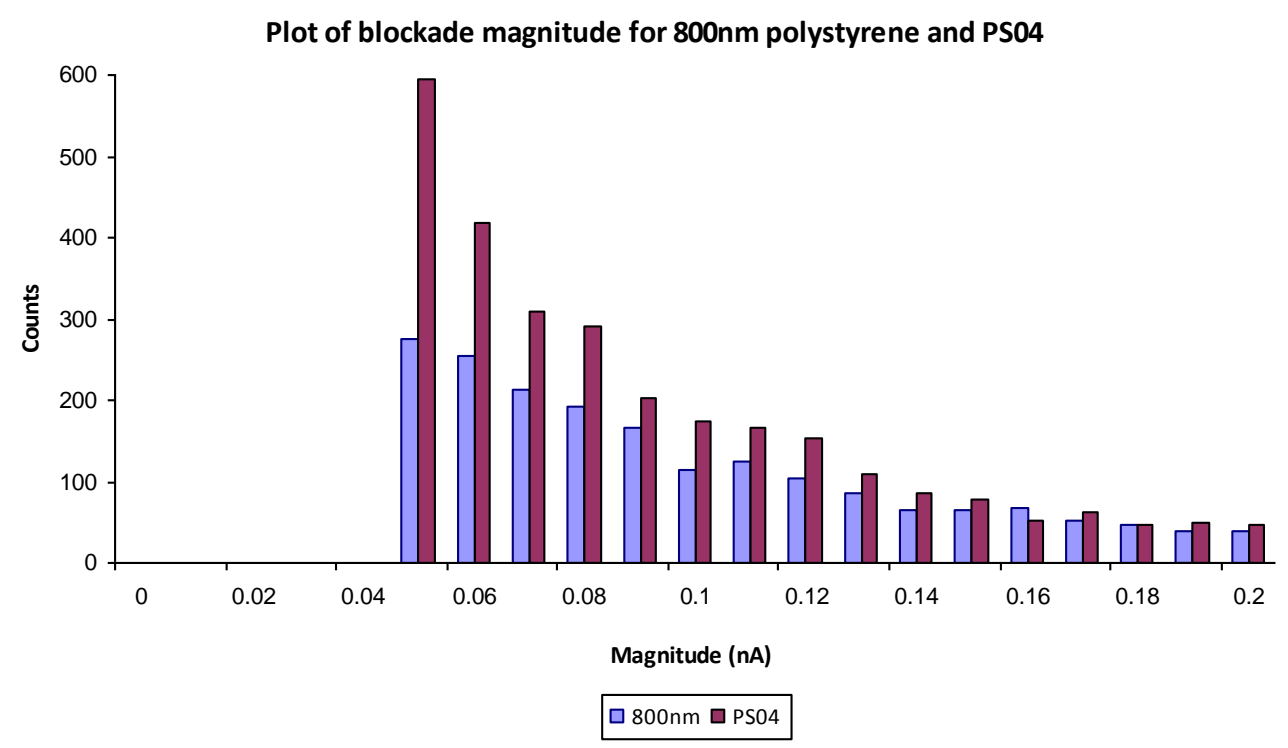

Figure 3.17: Histogram showing the distribution of blockade magnitudes for PSO4 and IZON's $800 \mathrm{~nm}$ polystyrene particles suspended in SEB.

Figure 3.17 shows the magnitude for the particles blockades for PSO4 and $800 \mathrm{~nm}$ polystyrene particles. Distribution profile for $800 \mathrm{~nm}$ and PSO4 particles' blockade magnitude does not differ much from each other due to the varying stretch of the pore. Signals from PSO4 (average diameter of $310 \mathrm{~nm}$ ) were done at $\Delta X Y=11.7 \mathrm{~mm}$ and $800 \mathrm{~nm}$ particles were done at $\Delta \mathrm{XY}=14.7 \mathrm{~mm}$. Since the pore needed to be stretched further, blockade magnitude recorded cannot be compared. The distribution however is still able to provide data in terms of the dispersity of the PSO4 and $800 \mathrm{~nm}$ samples used. An example of this can be shown by the distribution spread of PSO4 particles. The graph for PSO4 shows a distribution that tails at larger magnitudes. This indicates that the sample used has a broad distribution as shown in figure 3.11 where the standard deviation is approximately $16 \%$.

As been done for the distribution for the blockade magnitude, converting the $\mathrm{X}$-axis of figures 3.14 and 3.15 to a line distribution gives figures 3.18 and 3.19 . These plots show the particle blockades' duration distribution. 


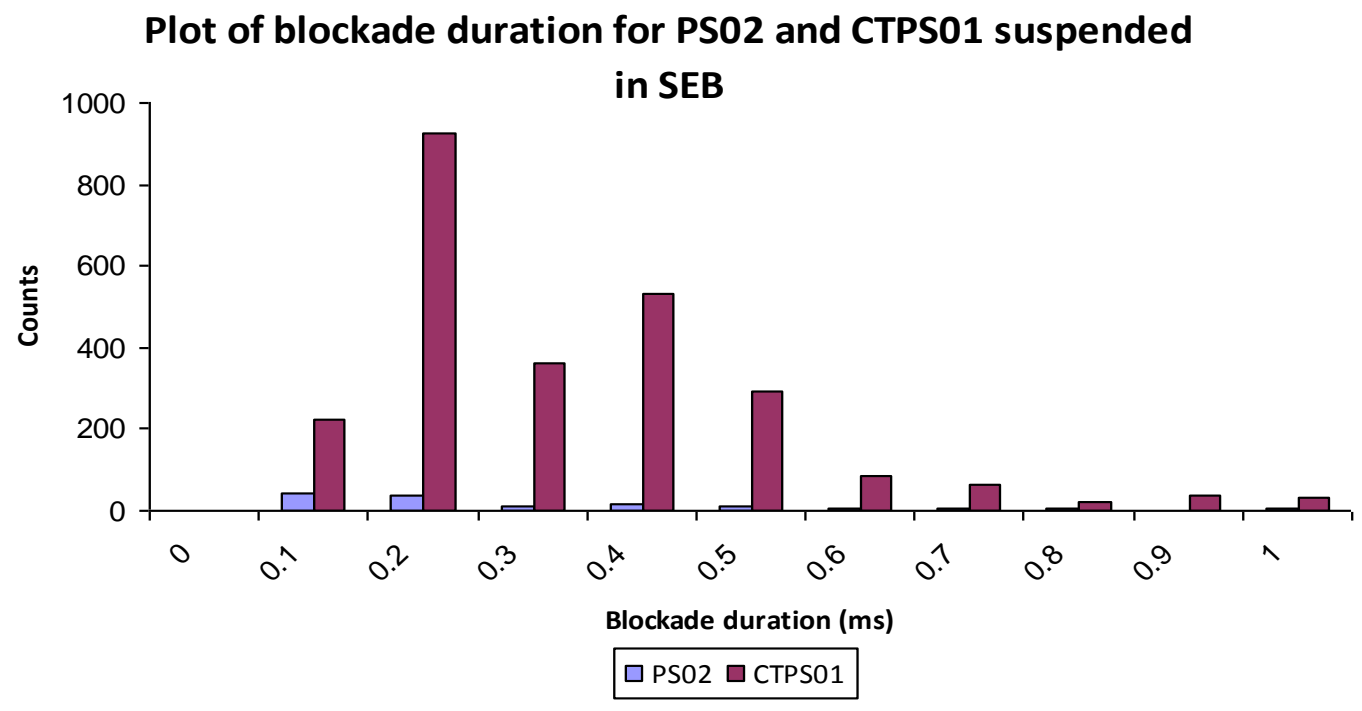

Figure 3.18: A histogram showing the distribution of blockade duration for both PSO2 and CTPSO1 particles suspended in SEB. Duration distribution for polystyrene particles peaks at a value of $0.1 \mathrm{~ms}$ and carboxylated particles 0.2 ms.

Figure 3.18 shows the distribution of blockade duration for PS02 and CTPS01. The figure shows that both sets of particle do not travel through the nanopore at the same rate as shown by the broad distribution. However, the majority of sample PSO2 travels faster than sample CTPSO1 with the duration of $0.1 \mathrm{~ms}$ and 0.2 ms respectively. Sample sets of IZON's $800 \mathrm{~nm}$ and PSO4 were also considered to analyse the trend of this observation. This is given in figure 3.19. 
Plot for blockade duration for 800nm and PSO4 suspended in SEB

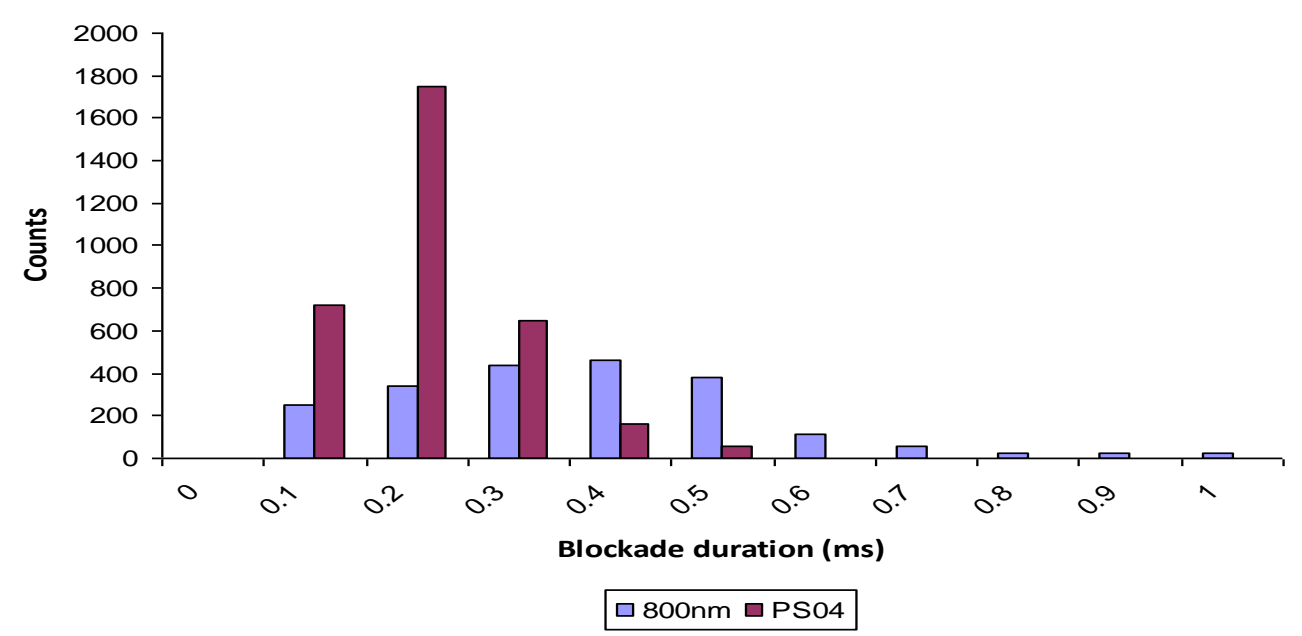

Figure 3.19: Histogram showing the distribution of blockade duration for both PSO4 and IZON's $800 \mathrm{~nm}$ polystyrene particles suspended in SEB. Translocation duration for polystyrene particles peaked at $0.2 \mathrm{~ms}$ and carboxylated particles at $0.4 \mathrm{~ms}$.

Figures 3.14 and 3.15 shows that blockade signals from PSO2 mostly lies in the region below $1 \mathrm{~ms}$ and $0.2 \mathrm{nA}$ and PSO4 lies within the region below $0.6 \mathrm{~ms}$ and $1 \mathrm{nA}$. This shows that the magnitude and duration signals from the particles are smaller in comparison to signals from CTPSO1 and $800 \mathrm{~nm}$ polystyrene. Figure 3.14 and 3.15 suggest that polystyrene particles travel through the pore faster than the carboxylated particles. This observation is supported by figures 3.18 and 3.19 where it can be seen that PSO2 peaks at $0.1 \mathrm{~ms}$ and PSO4 peaks $0.2 \mathrm{~ms}$, which is faster than CTPS01, which pass through the pore at $0.2 \mathrm{~ms}$ and $800 \mathrm{~nm}$ particles at $0.4 \mathrm{~ms}$.

\subsubsection{Discussion}

Carboxylated polystyrene particles usually have a higher surface charge compared to polystyrene particles [34, 97]. Results obtained from this experiment shows that the polystyrene particles travel faster than the carboxylated polystyrene particles for both cases. This is the opposite of the expected results where a greater surface charge would lead to faster translocation events [34]. This observation could be due to the changing pore stretch at which the measurements were conducted which will affect the translocation time of the particles. Equation (2) shows the relation between 
electrophoretic mobility, the length of the pore, the electric field, and the translocation duration. From equation 2, it can be seen that the electrophoretic mobility increases to the squared of the length of the nanopore. A study by Roberts et al. [97] showed that the nanopore experience membrane thinning when it is stretched further reducing the length of the nanopore by approximately $14 \%$. A decrease in the length means that the electrophoretic mobility with decrease to the power of two. This decrease in electrophoretic mobility in turn implies that the translocation duration will increase, as it is inversely proportional. Aperture that is fixed at a constant stretch is crucial for data comparisons between each sample set.

One of the results from this section also shows that by cross analysing the data obtained from SEM, SemAfore, and the qNano; it can be shown that qNano can serve as a tool to accurately compute the distribution of any sample. In addition to exhibiting the sample's distribution, it can also give the average particle diameter of a sample with the use of a calibration particle. This technique would give a much more accurate value as the data generated takes into account the diameter of every single particle present in the sample solution and not by averaging the diameter of the particles. This gives the qNano the extra advantage it needs and is extremely crucial to analyse sample that has a range of diameters in a solution. The only drawback to this technology regarding its use as a particle sizing instrument; is the difference in each aperture that was made. There is also the issue of aperture softening as it is used which would make data comparison invalid. However, this is not a major drawback as calibration of the aperture using the IZON standard particles can be done on each initial use of the aperture to ensure that the results are accurate each time.

\subsection{Measurements on qNano with nanoparticles}

Previous measurements of particles use those with a mean diameter of between 100 $\mathrm{nm}$ and $800 \mathrm{~nm}$ - which are considered to be submicron particles - due to the limitation of the aperture detection capability. Recent development of this technology has enabled nanoparticles - particles less than $100 \mathrm{~nm}$ - to be detected. Newer apertures are now made with a smaller needle to puncture the membrane used in making the apertures. In addition, the hardware in all of IZON's instruments has been 
upgraded to have a higher bandwidth i.e. from $10 \mathrm{kHz}$ to $50 \mathrm{kHz}$. Both of these developments open up a wider area of research now involving nanoparticles. A smaller aperture hole will tune its sensitivity to nanoparticles and a higher bandwidth means a more accurate nanoparticle resolution can be achieved. Measurements were taken on the instrument to demonstrate the ability to detect nanoparticles using upgraded technology.

\subsubsection{Experimental methods}

Both sets of particles used in this measurement were standard polystyrene particles as supplied by IZON - the $100 \mathrm{~nm}$ and $70 \mathrm{~nm}$ particles. These particles were suspended in SEB in a 1:500 dilution and sonicated for 20 minutes prior to its use. Voltage bias used in this measurement was $0.3 \mathrm{~V}$ and the magnitude threshold was set to a lower limit of $0.05 \mathrm{nA}$ and an upper limit of $1 \mathrm{nA}$ - termed [lower limit, upper limit] from here onwards.

\subsubsection{Results and Analysis}

IZON's new version of the hardware has enable detection of nanoparticles as the bandwidth is increased. Figure 3.20 shows the pattern of distribution for $100 \mathrm{~nm}$ and $70 \mathrm{~nm}$ polystyrene nanoparticles. 


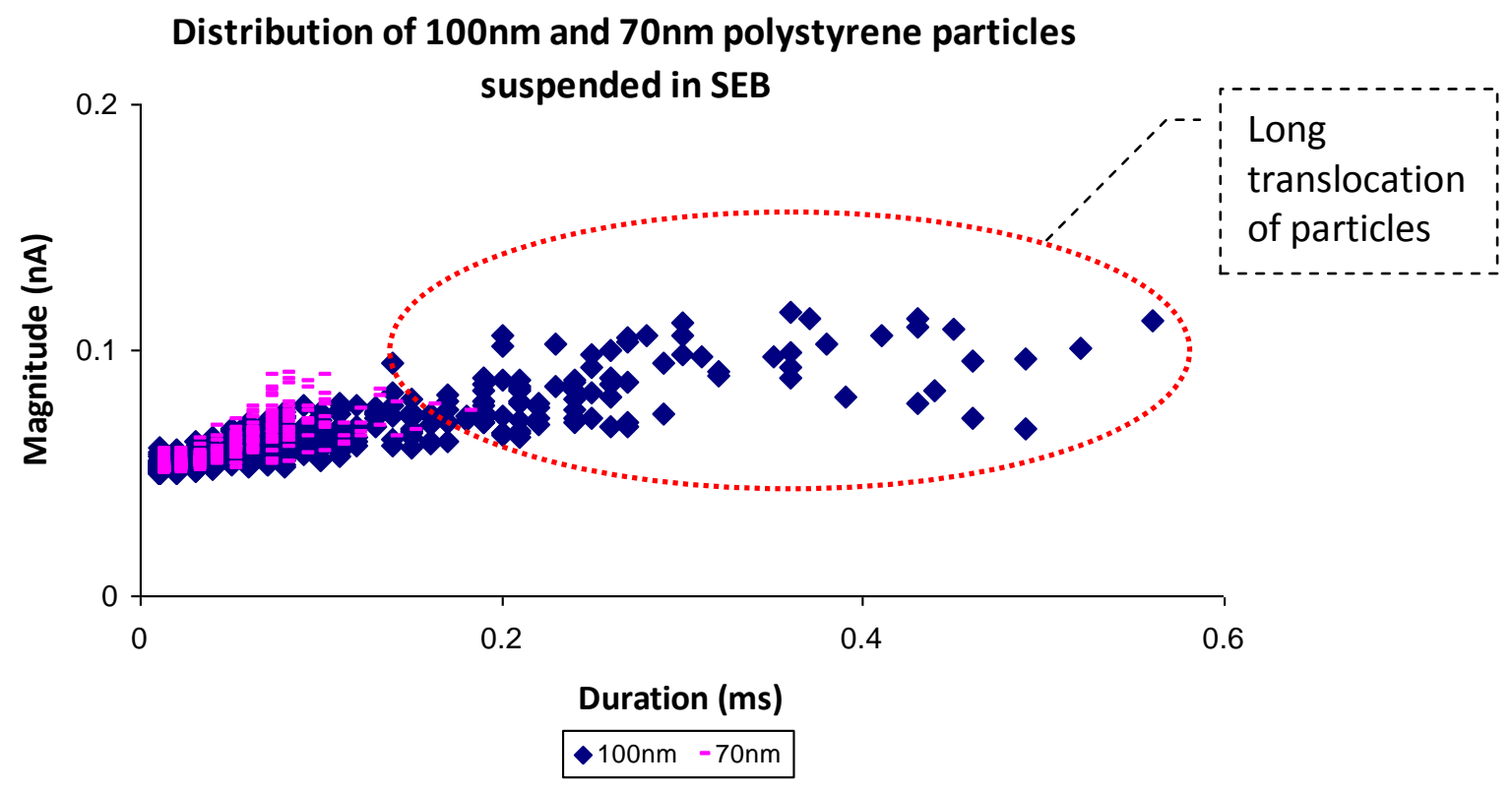

Figure 3.20: Scatter plot of particle blockades for both $100 \mathrm{~nm}$ and $70 \mathrm{~nm}$ polystyrene particles suspended in SEB. Figure shows that approximately $80 \%$ of blockade points from $100 \mathrm{~nm}$ polystyrene particles overlap with the blockade points of $70 \mathrm{~nm}$ particles, which suggest resolvability capability of the qNano system.

Figure 3.20 shows the distribution of particles blockades as it move through the nanopore. The plot shows that both particle sets mainly concentrate in the region of less than $0.1 \mathrm{~ms}$ in duration and between 0.05-0.1 $\mathrm{nA}$ in blockade magnitude. Data points in the red bubble show that some particles travels through the aperture at a much slower duration than majority of the particles. In terms of the average blockade magnitudes and duration, there is no noticeable difference between the two polystyrene sizes. This could be accounted to the change in aperture stretch as measurements were taken. Since the magnitude of particle blockade is relative to the size of the pore opening, a change in aperture stretch makes comparison challenging.

Figures 3.21 and 3.22 shows the screenshots from the software interface of blockades detected. These screenshots clearly shows a high frequency of particles translocation 760 counts for $100 \mathrm{~nm}$ and 497 counts for $70 \mathrm{~nm}$ particles were recorded in a space of 1 minute of measurement. 


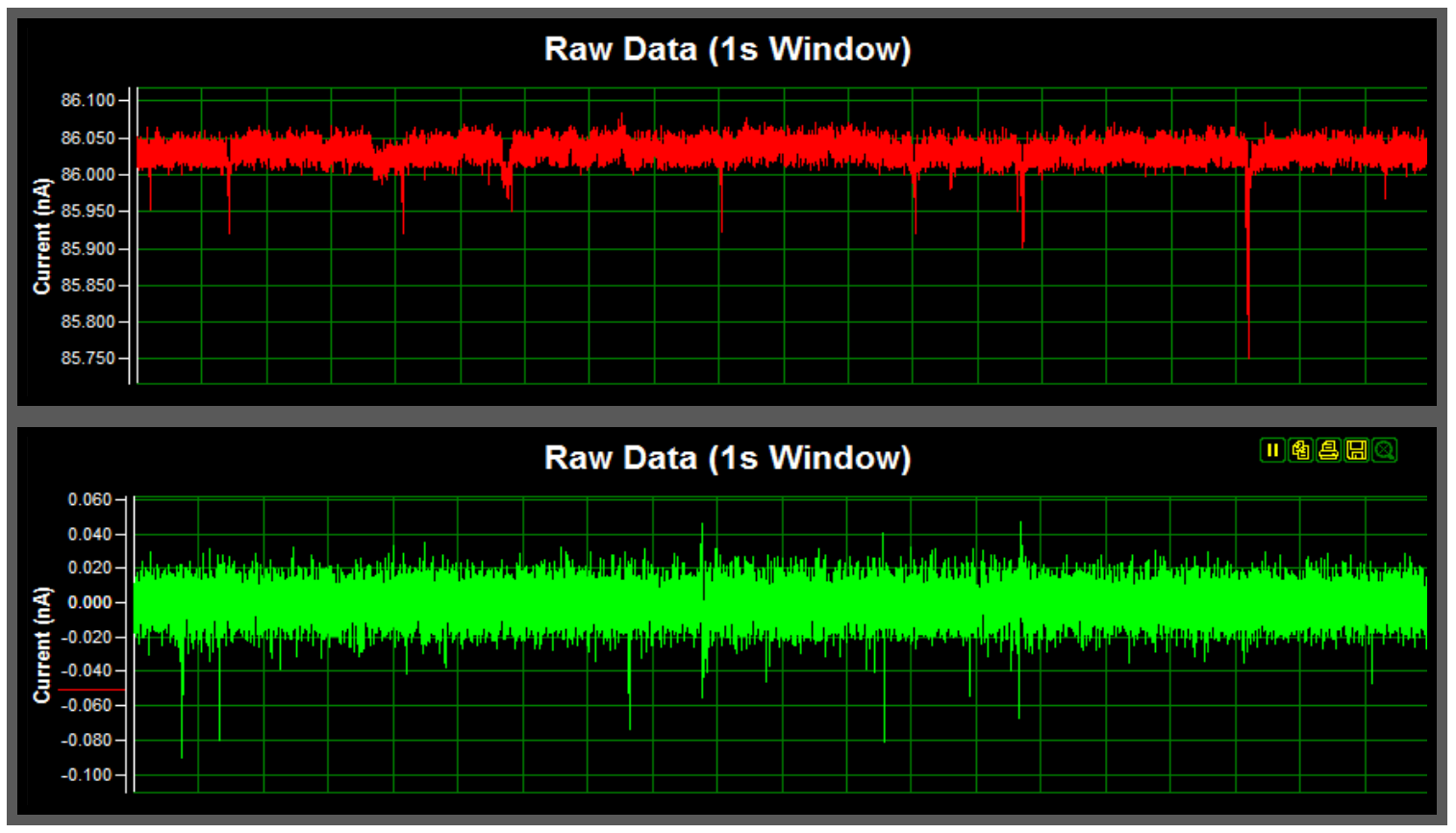

Figure 3.21: Screenshots for $100 \mathrm{~nm}$ blockades. The green window screenshot is on a normalised setting on the software that only takes the difference of the blockade magnitude with respect to the current baseline.

Figure 3.21 shows the screenshots for the translocation of $100 \mathrm{~nm}$ particles from the upper to the lower fluid cell. Figure 3.22 shows the screenshots for $70 \mathrm{~nm}$ particles.

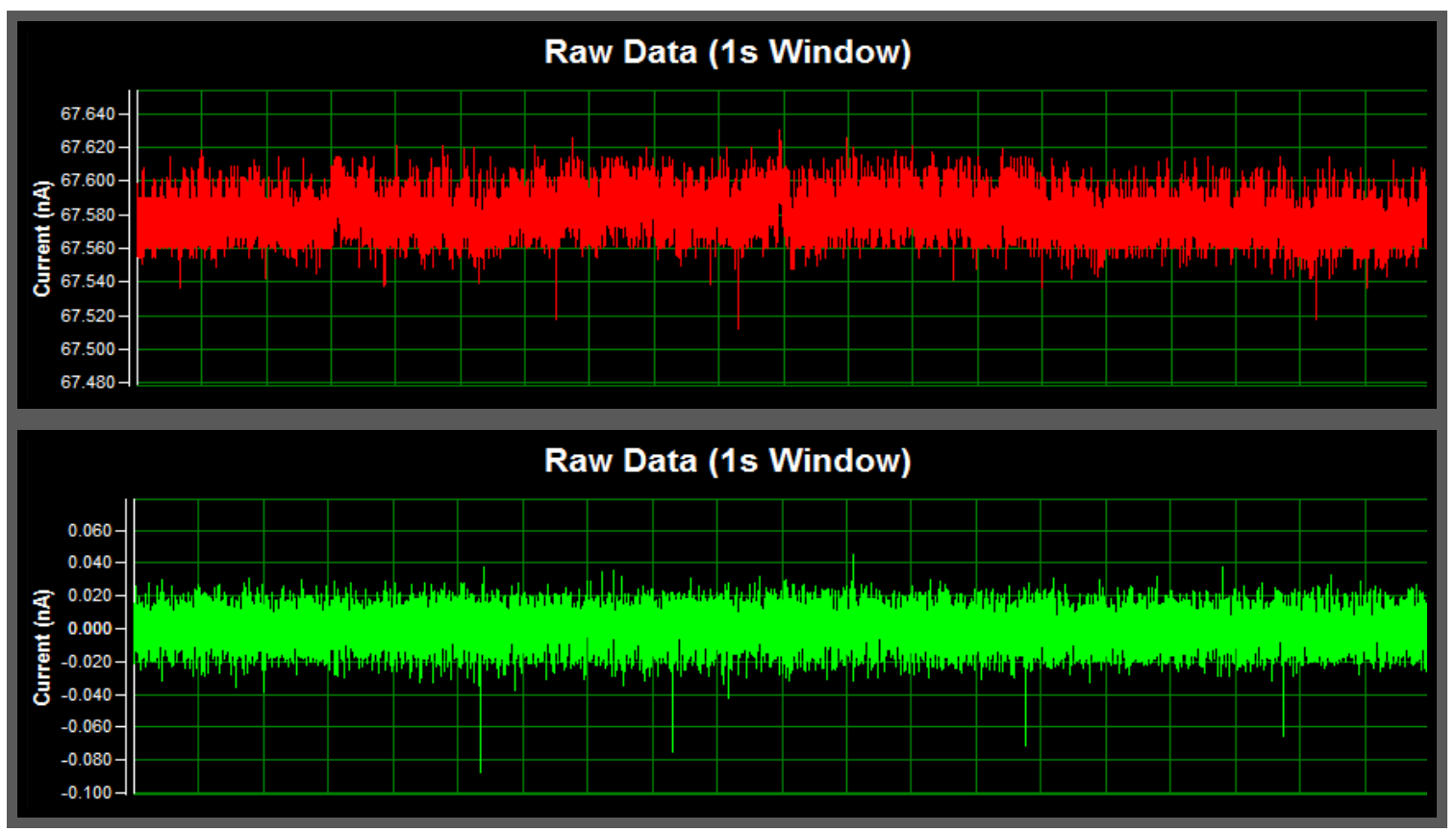

Figure 3.22: Screenshots for $70 \mathrm{~nm}$ blockades. The green window screenshot is on a normalised setting on the software that only takes the difference of the blockade magnitude with respect to the current baseline. 
From the normalised screenshots in figures 3.21 and 3.22, it can be seen that blockade magnitudes i.e. the downward spikes for both $100 \mathrm{~nm}$ and $70 \mathrm{~nm}$ particles are approximately between $0.04 \mathrm{nA}$ and $0.07 \mathrm{nA}$. Although the screenshots shows that the two sets of particles have a blockade magnitude that mirrors each other, it must be noted that the measurement was done at an aperture stretch that was not constant. The two set of particles were done at a stretch of $\Delta \mathrm{XY}=3.44 \mathrm{~mm}$ for $100 \mathrm{~nm}$ particles and $\Delta X Y=2.85 \mathrm{~mm}$ for $70 \mathrm{~nm}$ particles. This change has to be made, as running $70 \mathrm{~nm}$ particles at the same stretch of the $100 \mathrm{~nm}$ particles sees no blockades.

To analyse further the effect of particle diameters in the nano region, a histogram of blockade magnitude and duration was constructed as shown in figures 3.23 and 3.24.

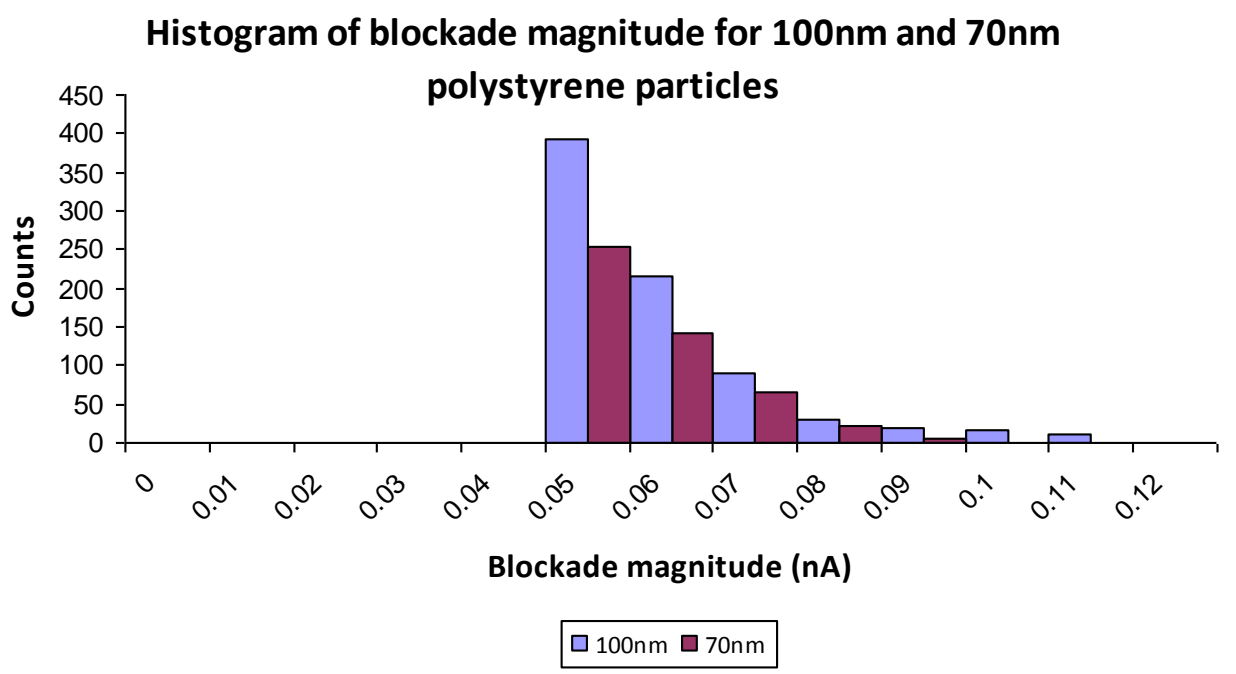

Figure 3.23: As the threshold settings were set at $0.05 \mathrm{nA}$ only particle blockade above this value will be recorded by the software. The histogram here depicts the distribution of both the $70 \mathrm{~nm}$ and $100 \mathrm{~nm}$ particles in the SEB.

Histogram in figure 3.23 shows that $100 \mathrm{~nm}$ and $70 \mathrm{~nm}$ particles have a mean value of blockade magnitude of $0.05 \mathrm{nA}$. The range of the histogram varies from $0.05 \mathrm{nA}$ to 0.11 $\mathrm{nA}$. Because the lower limit settings of the blockade magnitude is $0.05 \mathrm{nA}$, only particle blockades higher than this value will be recorded. A short right hand 'tail' of the histogram shows that the particles used are monodisperse. Note that because the lower magnitude threshold is high, there will be particles at the lower end of the 
distribution that will be 'drowned' by the noise signals. The duration histogram for particle movement is given in figure 3.24.

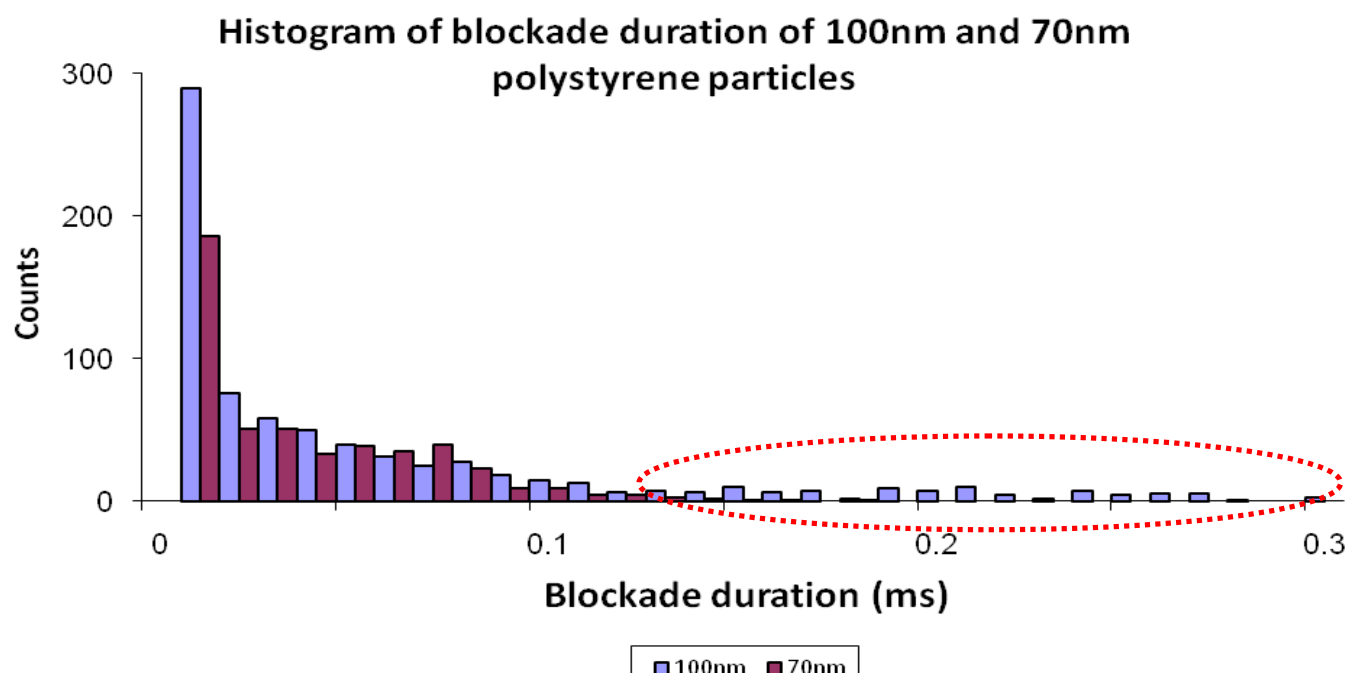

Figure 3.24: Histogram for blockade duration of $100 \mathrm{~nm}$ and $70 \mathrm{~nm}$ particles. The red bubble in figure 3.24 shows that there were particles that take a longer time traversing through the nanopore, which tallies with the distribution given in the red bubble of figure 3.20 .

Figure 3.24 now gives the distribution of particle translocation. The plot shows that the mean value of blockade duration is $0.01 \mathrm{~ms}$. The lower limit for duration threshold was set to 0 ms which means that blockades of any duration will be recorded. The advantage of setting this value to zero is that the software will not leave out any particles that go through the pore at a faster rate. At this stage, the verification that the data points that lies within the red bubble in figures 3.20 and 3.24 is purely of polystyrene particles and not any other sources is unknown.

\subsubsection{Discussion}

Data recorded in figures 3.20, 3.23 and 3.24 all shows that there were only a minor difference in blockade magnitude and duration for the particles measured. This is due to the change in aperture stretch that makes data comparison for these two sets of particles invalid as explained in section 3.2.3.4. Data measured from $100 \mathrm{~nm}$ to $70 \mathrm{~nm}$ particles requires the pore to be closed down, as $70 \mathrm{~nm}$ particles were 'invisible' to be detected if $\Delta \mathrm{XY}$ is remained constant. This is due to blockade signals from the smaller 
particles that are drowned by background noise. The dispersity of the particles could also affect the blockade counts as signals from $70 \mathrm{~nm}$ could be mistaken for the smaller sizes of the $100 \mathrm{~nm}$ particles sample.

The 'loss' of data could be caused by several factors. A big aperture hole at a low stretch limits the tuneability of the pore as explain in section 1.3 regarding the work of Willmott et al. [86]. Hence, due to the small size difference between the two set of particles, there is a restriction to the aperture stretch i.e. the working stretch window might overlap over each other. This limitation will especially surface as a problem in a used aperture. Because of the material used to make the aperture, the creep factor as demonstrate in section 3.2.1.1 will take into effect after a period of usage. On top of that, noisy background means a higher magnitude threshold setting which lessen the chances to detect the nanoparticle blockades.

\subsection{Summary}

This chapter was dedicated to the groundwork of how qNano operates as well as the study of qNano aperture. This includes the stability of IZON's aperture over a period of time (section 3.2.1.1), the technique and protocol used to detect particles in a sample (section 3.2.1.2) as well as showing the capability of the instrument to resolve different diameters of polystyrene particles - the bimodal distribution (section 3.2.2). Section 3.2.3 attempts to investigate the ability of the qNano instrument to resolve different species of polystyrene particles, however, further research could be conducted to substantiate the findings from this research. The ability for the instrument to detect inorganic nanoparticles means that detection of other nanoparticles such as metal nanoparticles is also a possibility. The next chapter will explore the measurement of gold nanoparticles using the qNano technology. 


\subsection{MEASUREMENT OF GOLD NANOPARTICLES}

\subsection{Introduction}

Gold nanoparticles are one of the most widely researched metal nano-materials due to the range of applications in electronics and the medical field [40 -43, 46, 47, 50, 53, 61]. This, coupled with the ease of synthesis, make them an ideal test system to work with. Due to the limitation of the aperture to detect nanoparticles smaller than $50 \mathrm{~nm}$, having nanoparticles in this small range is valuable as it can also be used to test the size limit of the instrument detection. To open up more platforms for researchers to characterise these nanoparticles it is important for instruments like qNano to be able to detect nanoparticles in this system. At present, no metal nanoparticles have been reported to be detected on qNano as most of the research done using this instrument focuses on areas related to biological research [89]. Therefore, it would be interesting to learn if qNano is able to detect metal nanoparticles, as this will boost its marketability.

\subsection{Synthesis of gold nanoparticles}

The simplicity of synthesizing gold nanoparticles makes the study of this metal very attractive. Synthesis of gold nanoparticles in this research uses the citrate reduction method. [37, 42, 46, 49, 65]. Gold ions present in the solution come from gold chloride $\left(\mathrm{HAuCl}_{4}\right)$. The gold ion, $\mathrm{Au}^{3+}$, is reduced by the citrate to form the gold atom $\mathrm{Au}^{0}$. The reduction process usually takes a few seconds to complete. This step produces the primary seed particles (less than $10 \mathrm{~nm}$ ) needed for the growth of gold nanoparticles [42].

The citrate ion used in this method has a dual function; to reduce the gold ion as well as acting as a stabilizer for the gold nanoparticles formed [35, 41, 57, 58]. The citrate ions have the carboxyl group on the exterior, which stabilizes the gold nanoparticles electrostatically [63]. This means that citrate ions adsorb themselves to the surface of the gold nanoparticles, creating an electrical double layer $[52,55,57,58,59]$. 
Apart from the ease of making gold nanoparticles with this method, the other reason it is preferable is that it has water as a solvent, which works best with IZON's standard buffer solution. The hydrophilicity of gold nanoparticles synthesized with this method means that no further steps such as ligand exchange are required to render the nanoparticles water soluble, which is the case with most other nanoparticles available.

\subsubsection{Method}

Gold nanoparticles in this research were synthesized using the citrate reduction method $[37,42,46,49,65]$. A few variations of this method were employed in synthesizing the gold nanoparticles and the reaction conditions are given in Table 4.1.

\begin{tabular}{|l|l|l|l|l|}
\hline Experiment & Trial & $\begin{array}{l}\text { Temp. } \\
\left({ }^{\circ} \mathrm{C}\right)\end{array}$ & $\begin{array}{l}\text { Conc. of gold } \\
\text { chloride }(\mathrm{mM})\end{array}$ & $\begin{array}{l}\text { Conc. of } \\
\text { sodium } \\
\text { citrate }(\mathrm{mM})\end{array}$ \\
\hline 1 & $\mathrm{a}$ & 80 & 0.396 & 1.69 \\
\hline & b & 80 & 3.960 & 2.56 \\
\hline 2 & a & 80 & 0.511 & 0.68 \\
\cline { 2 - 5 } & b & 80 & 0.642 & 0.71 \\
\hline 3 & - & 100 & 0.340 & 3.17 \\
\hline 4 & - & 100 & 0.191 & 0.88 \\
\hline 5 & a & 50 & 1.04 & 1.43 \\
\cline { 2 - 5 } & b & 20 & 1.02 & 1.29 \\
\hline
\end{tabular}

Table 4.1: Reaction conditions in synthesizing gold nanoparticles by citrate reduction method.

Table 4.1 shows a summary of reaction conditions used to synthesize the gold nanoparticles used in this study. To make the gold nanoparticle samples for this study, gold chloride $\left(\mathrm{HAuCl}_{4} \cdot 3 \mathrm{H}_{2} \mathrm{O}\right)$ was dissolved in deionised water. The solution was then heated up with vigorous magnetic stirring. A solution of sodium citrate ( $\mathrm{Na}-\mathrm{Cit}$ ) was then added drop-wise to the hot gold solution. At this point, the solution will change its colour from yellow to purple-pink. The heating element was immediately turned off 
after the colour change but the solution was stirred for a further five minutes or until there is no colour change and allowed to cool down to room temperature. A typical colour change for the solution is yellow $\rightarrow$ grey $\rightarrow$ dark blue $\rightarrow$ purple $\rightarrow$ deep purple $\rightarrow$ red. All samples were purified by centrifuging at 10,000 rpm for 1 hour.

\subsubsection{Risk assessments}

Table 4.2 lists the associated dangers [91] of the chemicals that were used to synthesize the gold nanoparticles.

\begin{tabular}{|c|c|c|}
\hline Chemicals & Risk & Danger \\
\hline \multirow[t]{4}{*}{$\mathrm{HAuCl}_{4} \cdot 3 \mathrm{H}_{2} \mathrm{O}$} & \multirow[t]{4}{*}{ Corrosive } & $\begin{array}{l}\text { Causes severe burns; irritating to the eyes (and } \\
\text { risking severe damage) respiratory system and } \\
\text { skin. }\end{array}$ \\
\hline & & Limited evidence of a carcinogenic effect. \\
\hline & & May cause sensitization by inhalation. \\
\hline & & May cause sensitization by skin contact \\
\hline Na-Citrate & Average & $\begin{array}{l}\text { May cause irritation to skin, eyes, and } \\
\text { respiratory tract. }\end{array}$ \\
\hline
\end{tabular}

Table 4.2: Risk evaluation

\subsubsection{Results and Analysis}

All gold nanoparticles were characterised with Transmission Electron Microscopy (TEM), Scanning Electron Microscopy (SEM), and Energy Dispersive Spectroscopy (EDS) for images of the nanoparticles. Their optical properties were characterised via UV-Vis Spectroscopy using a Cary 100 Scan UV-Vis Spectrophotometer.

\subsubsection{Experiments $1(\mathrm{a})$ and $1(\mathrm{~b})$}

\section{$\underline{\text { Results }}$}

The variables manipulated in this experiment are the concentration of both gold precursor and sodium citrate used. The difference in sodium citrate used between the two experiments was approximately $0.1 \mathrm{ml}$ and the concentration of gold used in 
experiment 1 (b) was ten times more than that in experiment 1 (a), i.e. $3.96 \mathrm{mM}$ and $0.396 \mathrm{mM}$ respectively. Images of the gold nanoparticles synthesized for experiments $1(\mathrm{a})$ and $1(\mathrm{~b})$ are given in figure 4.1 below.

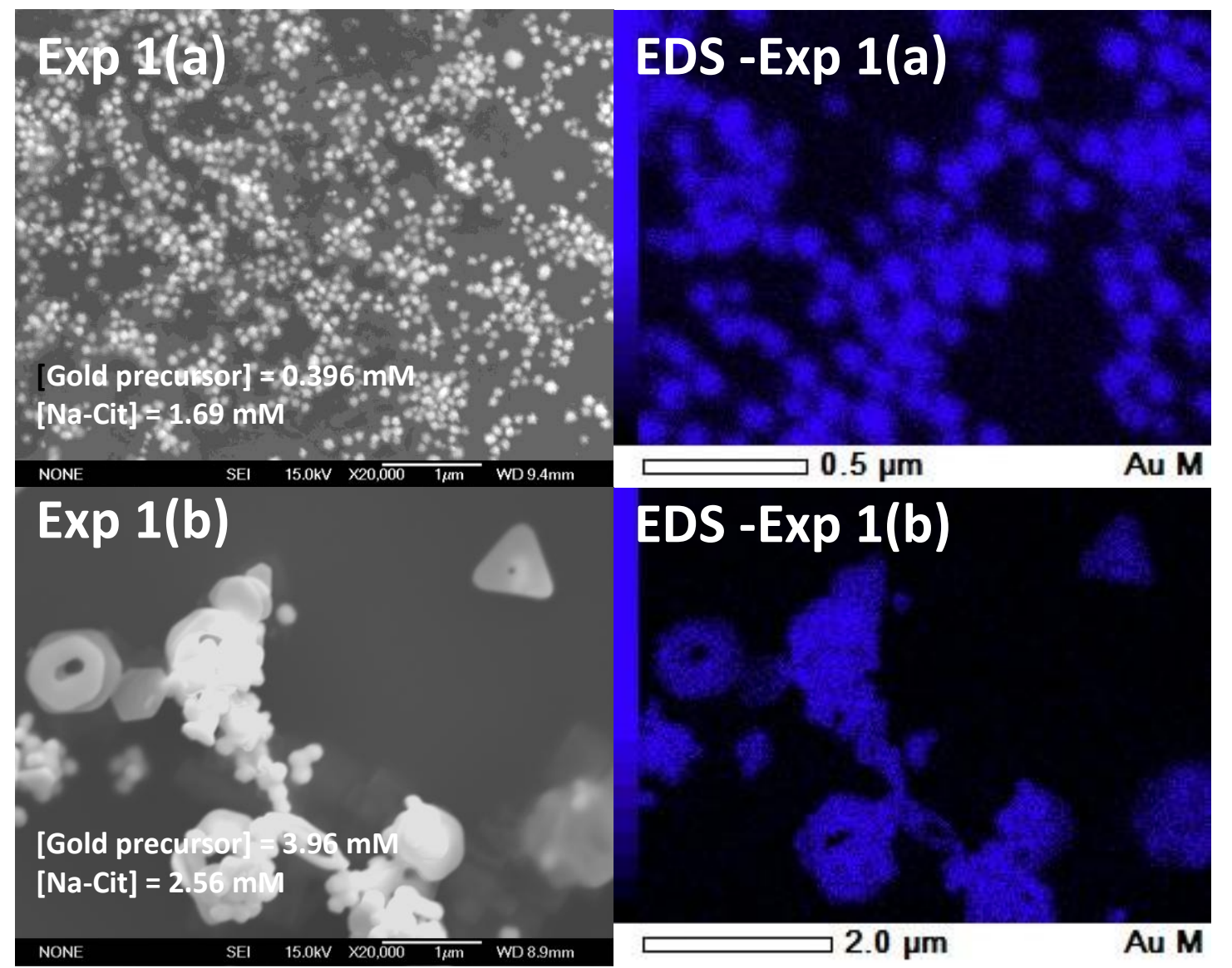

Figure 4.1: Images for 1(a) and 1(b) gold nanoparticles and their respective EDS mapping image at $\times 20,000$ magnification. Experiment $1(b)$ has a ten-fold increase in the concentration of gold precursor and 50\% increase in the concentration of sodium citrate as compared to Experiment 1(a). A more irregular shape is produced with the conditions of Experiment 1(b). The EDS confirms that the particles imaged are gold nanoparticles.

Figure 4.1 shows two very different shapes of nanoparticles synthesized using method 1 (refer to Table 4.1). The image of experiment 1(a) shows the expected results for the gold nanoparticles synthesized. The nanoparticles are mostly spherical in shape. Sample 1(b) however shows a very different shape distribution as compared to the sample 1(a). It can be seen that this sample has multi-faceted as well as spherical nanoparticles. This kind of sample was also reported by the works of Turkevich et al. 
[37]. The distribution of the nanoparticles was built using JEOL's SemAfore, a digital software that was used to manually count and size the nanoparticles. Usually, an image taken from either TEM or the SEM is used. Measuring tools available on the software utilise the scale bar present on the image and nanoparticles are individually counted and manually sized. Figure 4.2 gives the distribution of samples from experiment $1(\mathrm{a})$.

Size distribution of gold particles from Exp 1(a)

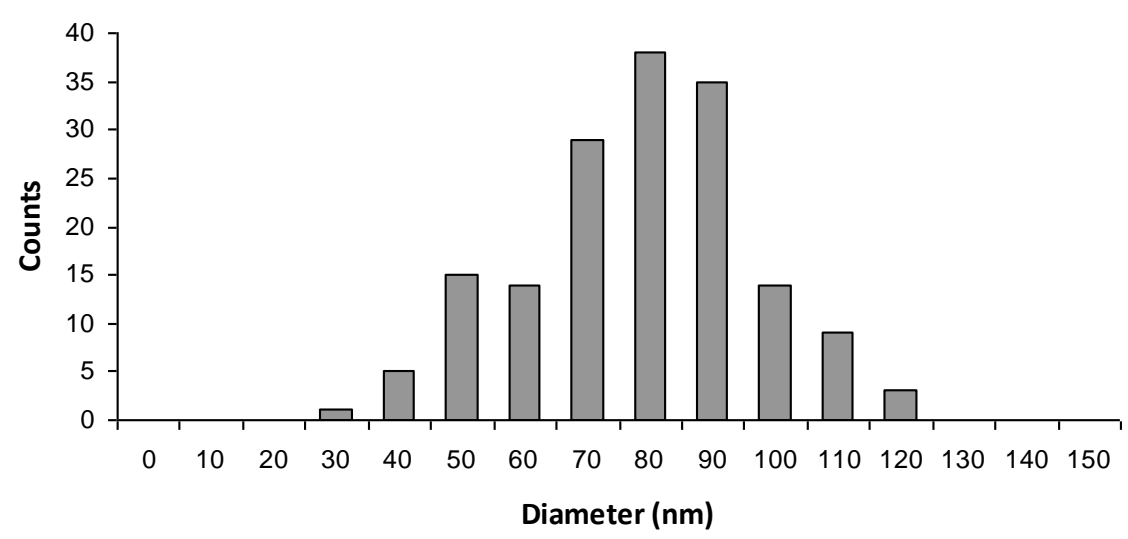

Figure 4.2: Size distribution for samples of experiment 1(a). The distribution gives a mean value of $80 \mathrm{~nm} \pm 45 \mathrm{~nm}$.

The histogram generated in figure 4.2 shows that the gold nanoparticles have a mean diameter of $80 \mathrm{~nm}$ with a broad standard deviation of $45 \mathrm{~nm}$. This is a very polydispersed sample but at this stage, the dispersity of the sample is not crucial, as any signals of gold would be of interest. The average diameter of sample 1(a) is supported by the peak of approximately $575 \mathrm{~nm}$ of the UV-Vis spectrum as given in figure 4.3. 


\section{Absorbance peak of gold nanoparticles from Exp 1(a)}

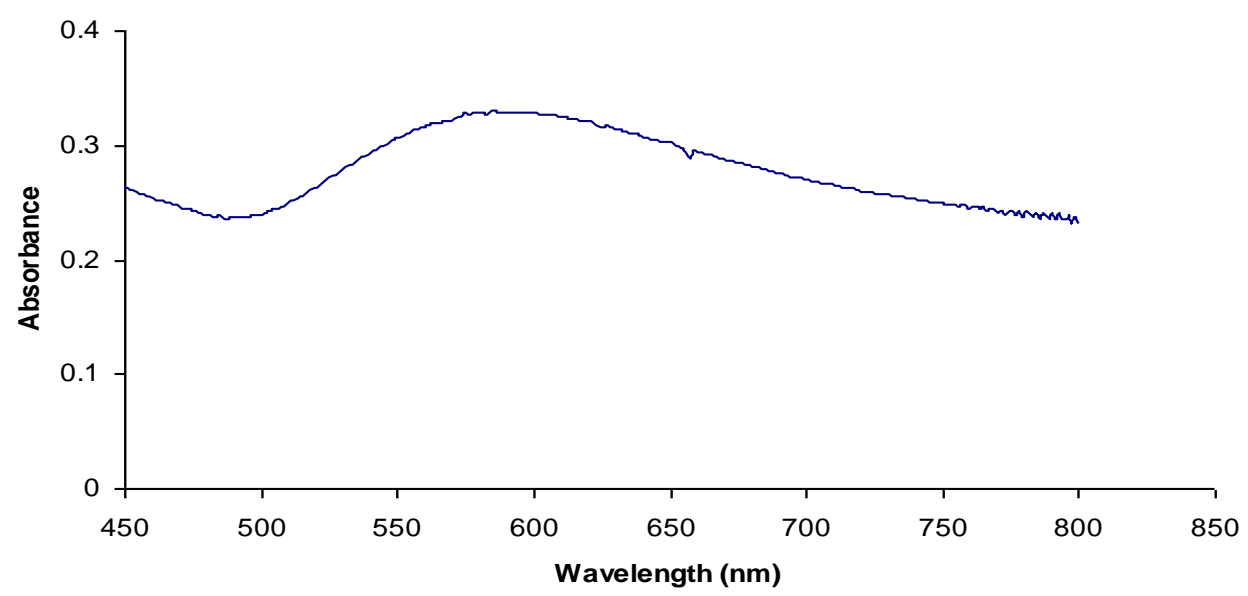

Figure 4.3: Absorption peak for gold nanoparticles form experiment 1(a). The plot has a peak of approximately $575 \mathrm{~nm}$ that indicates the presence of aggregates in the sample.

Figure 4.3 shows the absorbance spectrum of the gold nanoparticles produced. It showed that the absorbance has a peak at approximately $575 \mathrm{~nm}$.

\section{$\underline{\text { Discussion }}$}

The major difference between samples $1(a)$ and $1(b)$ is the concentration of the gold precursor used. Sample 1 (b) has a ten-fold gold concentration compared to sample 1(a). The concentration of sodium citrate used between the two however has a difference of only $0.6 \mathrm{mM}$. As shown in figure 4.1 , reaction conditions for sample 1 (b) produced multi-faceted nanoparticles. This demonstrates that the concentration of sodium citrate used was insufficient for producing monodisperse spherical gold nanoparticles. Furthermore, because the gold chloride ion are in excess (compared to the citrate ion); the nanoparticles produced are likely to be of a larger diameter. This is because there is a limited amount of capping agent (citrate ion) available to stabilize the gold nanoparticles formed in the solution [78]. Henglein and Giersig [78] shows synthesis of silver nanoparticles capped with citrate ions produce nanoparticles that are large and aggregated with a wide size distribution. This finding is also true at high concentration of citrate ions as at high ionic strength, citrate ions destabilize as it shows to form large cluster of silver nanoparticles. 
The absorbance peak for sample $1(a)$ is approximately $575 \mathrm{~nm}$. This is slightly higher than the expected peak of gold nanoparticles suspended in aqueous media, which is typically around $524 \mathrm{~nm}$ [35, 38, 39, 49, 50, 59]. However, Kimling et al., [42] reported that an absorbance peak for $85 \mathrm{~nm}$ diameter nanoparticles was observed at approximately $550 \mathrm{~nm}$. In another work by Daniel and Astruc [53], nanoparticles with an average diameter of $9 \mathrm{~nm}, 15 \mathrm{~nm}, 22 \mathrm{~nm}, 48 \mathrm{~nm}$, and $99 \mathrm{~nm}$ correspond to absorbance peaks situated at $517 \mathrm{~nm}, 520 \mathrm{~nm}, 521 \mathrm{~nm}, 533 \mathrm{~nm}$, and $575 \mathrm{~nm}$ respectively [53]. A red shift in the peak of the absorbance spectrum usually indicates larger particle diameter [36, 40, 59]. Brown et al., [40] reported that an absorbance peak of more than $670 \mathrm{~nm}$ usually indicates presence of gold aggregates in the solution.

\subsubsection{Experiment 2(a) and 2(b)}

Sample 1(a) showed a sample that is spherical with a mean diameter of $80 \mathrm{~nm}$. Further analysis of sample 1(a) showed that the particles are aggregates. It is preferable that the samples used for measurements on qNano to be single particles. Therefore, the method developed by Turkevich et al. [37] was attempted.

\section{$\underline{\text { Results }}$}

In Experiments 2(a) and 2(b), only the concentration of gold precursor used was varied when synthesizing nanoparticles with this method. Experiment 2(a) used $0.511 \mathrm{mM}$ while experiment 2(b) used $0.642 \mathrm{mM}$. The amount of sodium citrate used for both of these syntheses is $0.04 \mathrm{ml}$ of a $0.34 \mathrm{M}$ solution. The scanning electron microscopy (SEM) images of the results given in figure 4.4. 


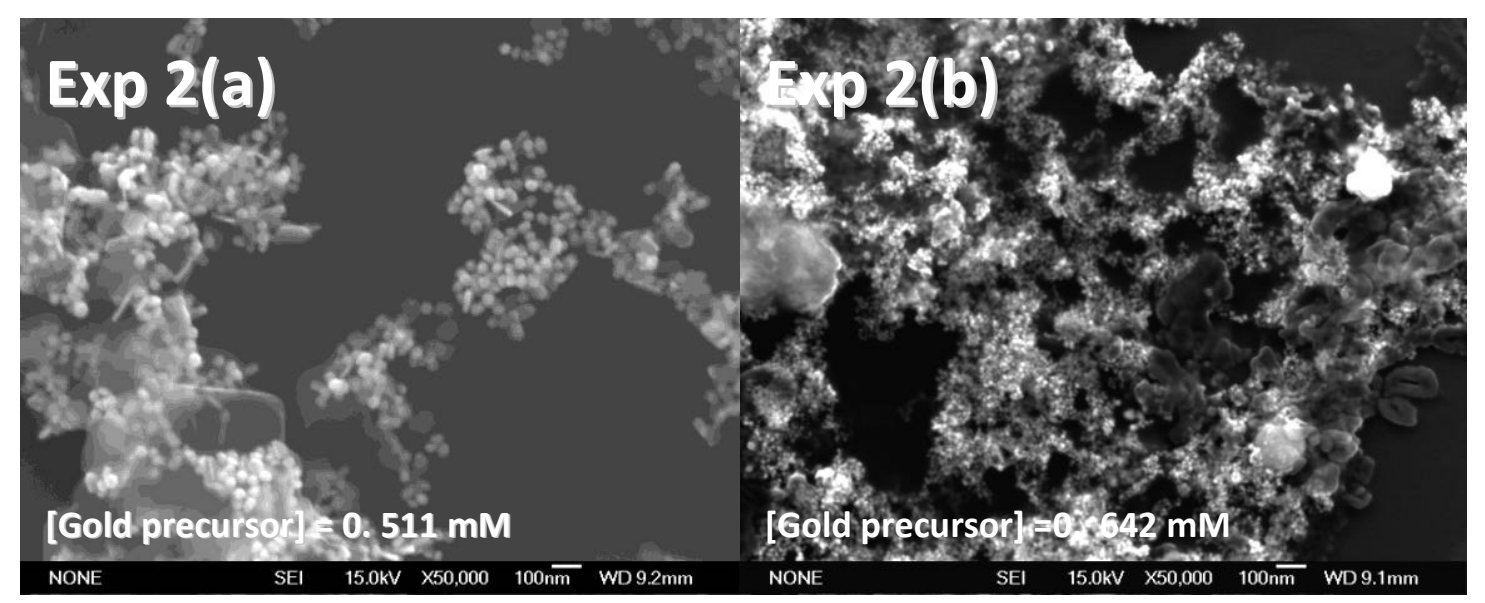

Figure 4.4: SEM images for 2(a) and 2(b) gold nanoparticles. Imaged at $x 50,000$ magnification. Experiment 2(b) has a $20 \%$ increase in the concentration of gold precursor compared to Experiment 2(a). Both samples show a network of gold particles synthesized.

As can be seen in figure 4.4, this method produced samples that form aggregates with a very polydispersed distribution. TEM images for experiments $2(a)$ and 2(b) show that a network of nanoparticles form which suggests that there were not enough citrate ions available in the solution for spherical gold nanoparticles to form. Although the sample produced was not monodispersed, there is no doubt that these were gold nanoparticles as confirmed by EDS mapping as shown in figure 4.5. 


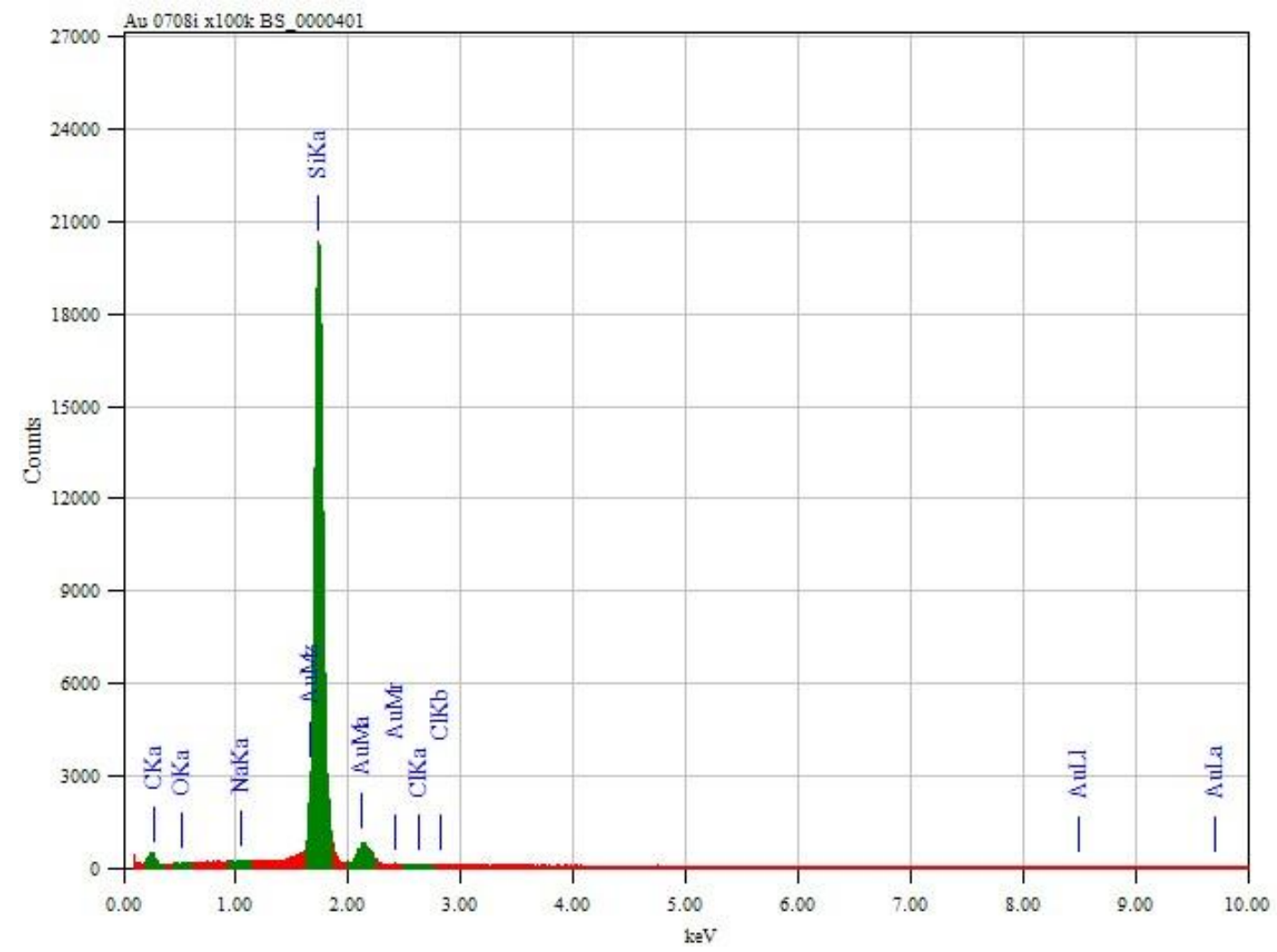

Figure 4.5: EDS mapping of a selected spot of sample from experiment 2(a). The EDS plot shows a notable peak for gold which confirms that gold nanoparticles are present in the sample.

Figure 4.5 shows a strong main peak for the gold element with presence of its satellites. This confirms that the nanoparticles that were seen are gold nanoparticles. Further characterisation uses gold's optical properties via UV-Vis Spectrometry. Figures $4.6 \mathrm{a}$ and $4.6 \mathrm{~b}$ show the absorbance spectrum for nanoparticles from experiments 2(a) and 2(b). 


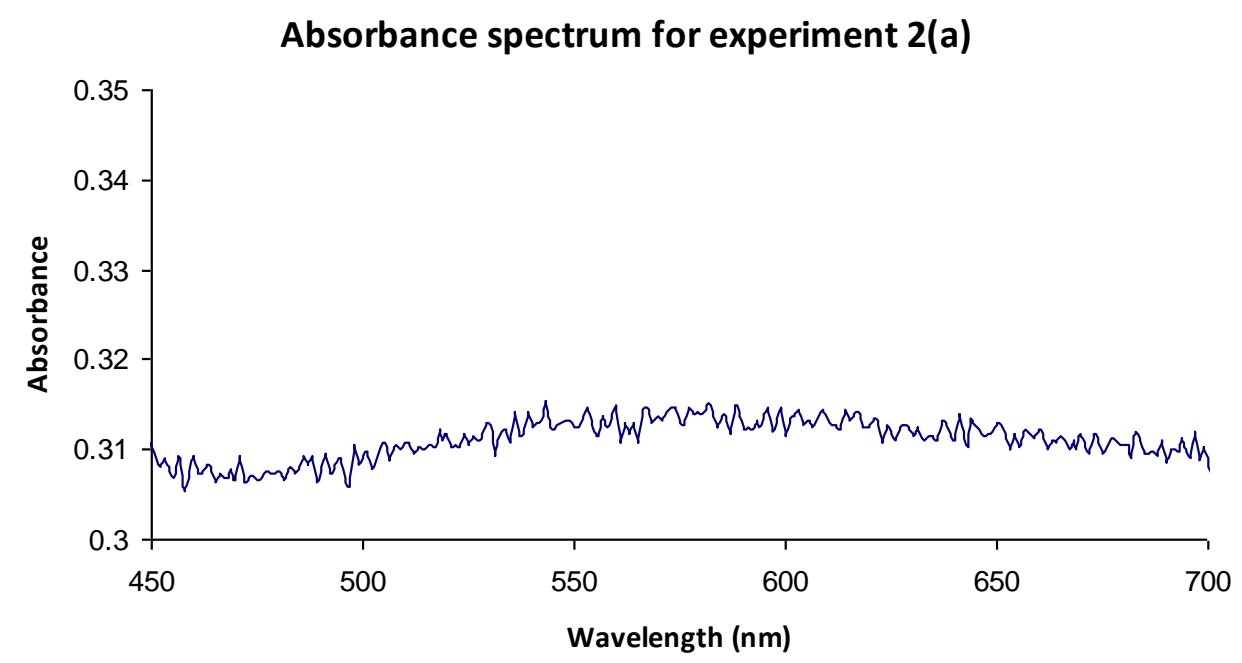

(a)

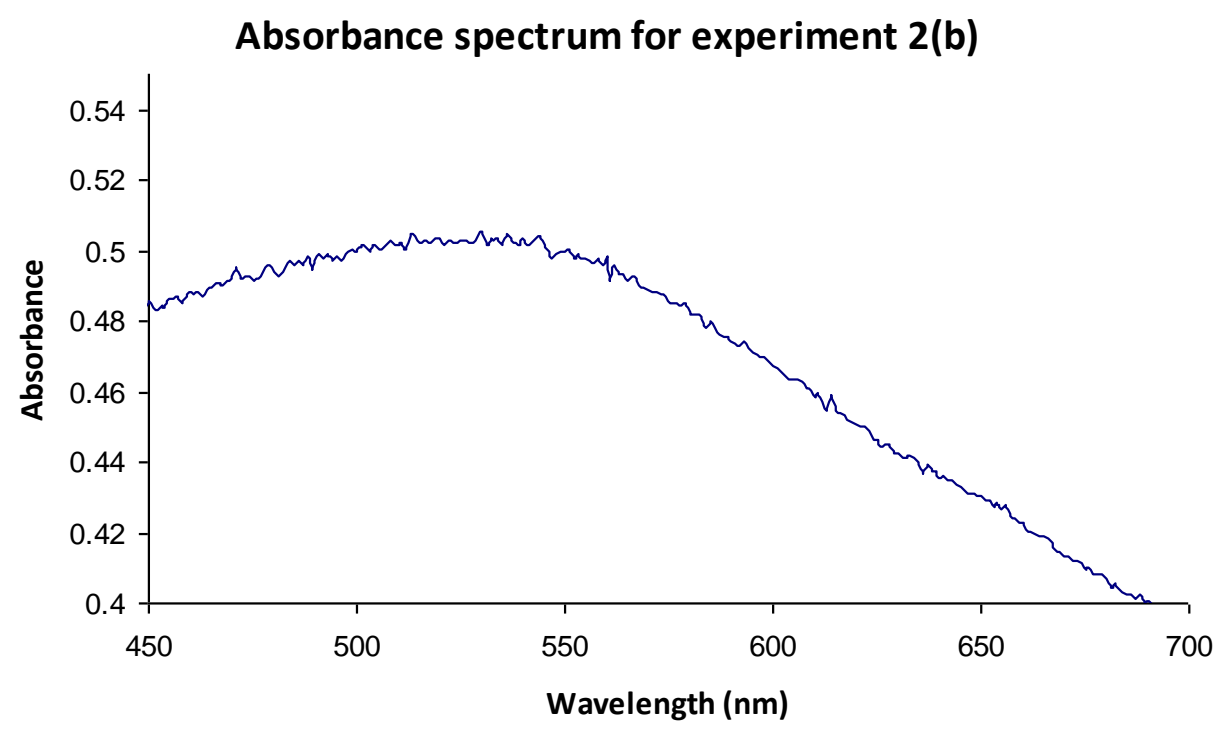

(b)

Figure 4.6: UV-Vis absorbance spectrum on both nanoparticles from experiments 2(a) and 2(b), which peaked at a region of $550 \mathrm{~nm}$, a feature of the gold nanoparticles.

As shown in figure 4.6, the signal output for these two nanoparticles is weak as shown by the low magnitude of the absorption peak. Regardless, both of these nanoparticles produce a signal that peaks within the $550 \mathrm{~nm}$ region, which is a characteristic of gold nanoparticles. Therefore, it can be assumed with confidence that the nanoparticles synthesized are gold nanoparticles. 


\section{Discussion}

Because the amount of sodium citrate used was kept as a constant, i.e. $0.04 \mathrm{ml}$ of 0.34 $M$ solution, larger gold nanoparticles were expected with the higher concentration of gold precursor used. However, as seen in the image of experiment 2(b) in figure 4.4, no specific shape was formed. Instead, networks of what is assumed to be gold nanoparticles were synthesized. This implies that a higher concentration of citrate ions is needed for gold concentrations of more than $0.5 \mathrm{mM}$. Results from experiment 1 (a) seem to suggest that the most effective concentration ratio of gold chloride ions to citrate ions is approximately 1:4. This ratio is used to justify the presence of gold aggregates in the sample. Therefore, due to the absence of monodisperse gold nanoparticles, this sample will not be used in further measurements on the qNano.

\subsubsection{Experiments 3 and 4}

Due to the unsuccessful attempt in experiment 2, more method options were explored. Two of these are taken from Mine et al. [46] (experiment 3) and Sugunan et al. (experiment 4) [49]. These methods differ in the concentration of both gold precursor and sodium citrate used.

\section{$\underline{\text { Results }}$}

Experiment 3 uses a higher concentration of gold precursor, i.e. $3.4 \mathrm{mM}$ compared to $1.9 \mathrm{mM}$ for experiment 4 , and $3.17 \mathrm{mM}$ of sodium citrate compared to $0.88 \mathrm{mM}$ for experiment 4. The results for nanoparticles made using this method are given in figure 4.7. 


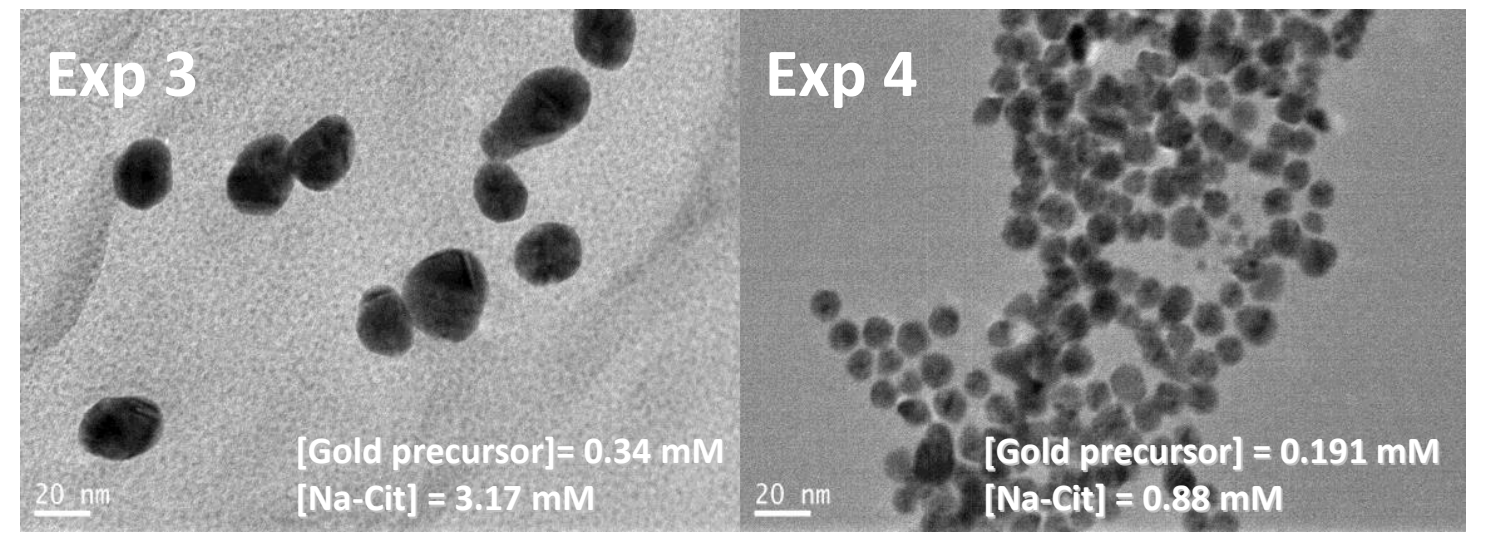

Figure 4.7: TEM images for gold nanoparticles from experiment 3 and experiment 4. Experiment 3 uses a ratio of sodium citrate to gold precursor that is much higher than those used in Experiment 4. Both samples show gold nanoparticles that exist as single particles in the solution.

Figure 4.7 shows the results from synthesis using methods 3 and 4 . It can be seen that these methods produce almost monodispersed, spherical nanoparticles. The ratio of gold precursor to citrate ions used in these experiments is over the recommended ratio of 1:4 for both sets of experiments. As can be seen, higher citrate ion to gold ratios produce nanoparticles that are spherical and evenly distributed. Experiment 3 has a citrate ion to gold ratio that is almost two fold compared to samples from experiment 4 . This doubled ratio proves that it makes nanoparticles that are much more isolated and not aggregating. However, from the image taken, it seems that the nanoparticles have a broad distribution of sizes. This is supported by the size distribution collected using the SemAfore software, a digital image analysis software that was used to manually analyse the TEM image of the nanoparticles. The size distributions of these nanoparticles are given in figure 4.8 and figure 4.9. 


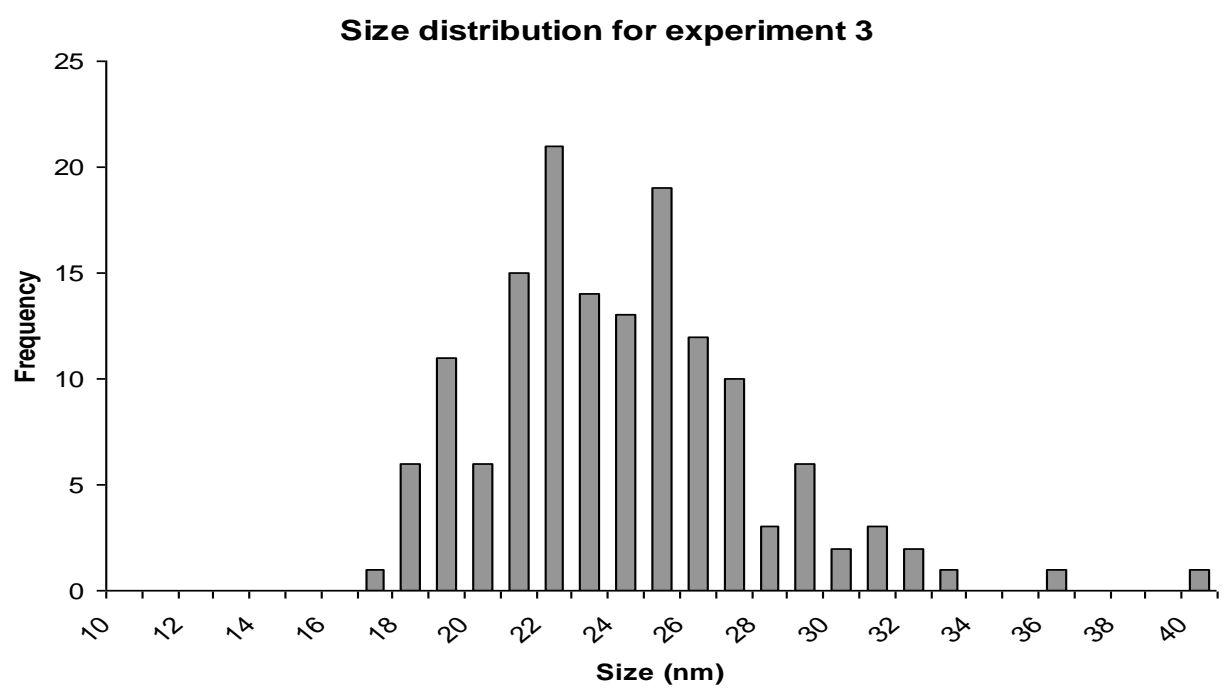

Figure 4.8: Size distribution of sample from experiment 3 shows that the sample has an average diameter of $22 \mathrm{~nm} \pm 12 \mathrm{~nm}$.

Figure 4.8 shows the size distribution of experiment 3 as analysed using SemAfore. It can be seen that the average diameter of the nanoparticles is approximately $22 \mathrm{~nm} \pm$ $12 \mathrm{~nm}$. The standard deviation for the size is more than $50 \%$. Therefore, it can be said that this sample is polydispersed. Figure 4.9 shows the size distribution of experiment 4.

\section{Size distribution for Experiment 4}

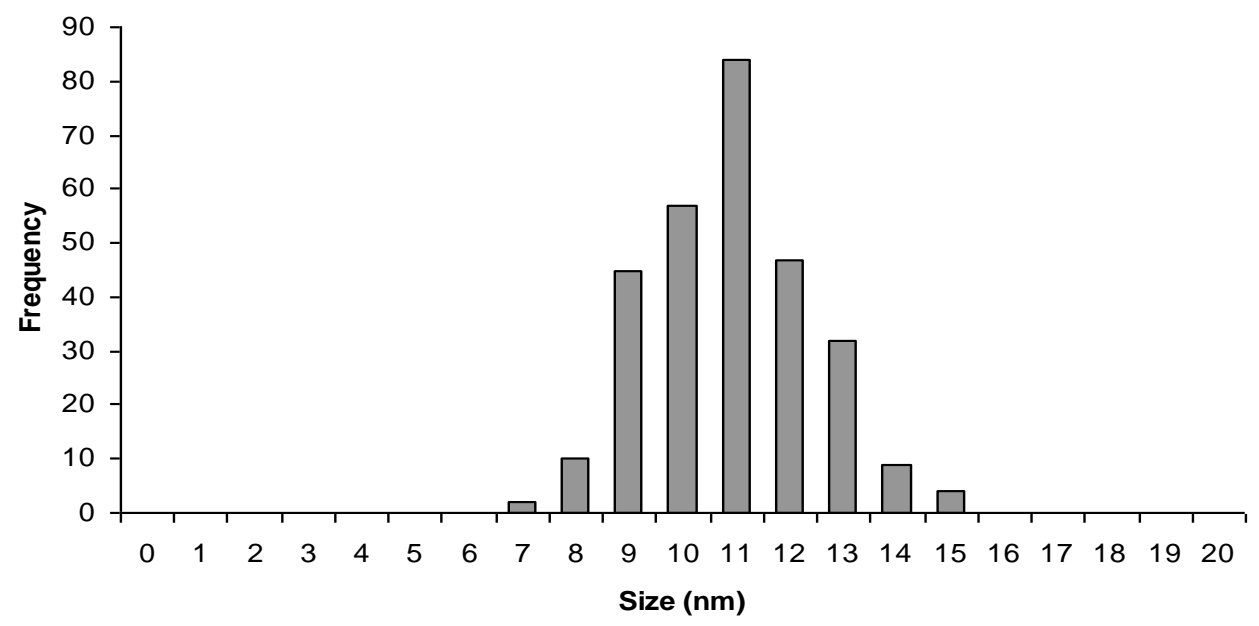

Figure 4.9: Size distribution of sample 4 shows that the sample has an average diameter of $11 \mathrm{~nm} \pm 4 \mathrm{~nm}$. 
Figure 4.9 shows a histogram of the size distribution of experiment 4 . It can be seen that the average diameter of experiment 4 is $11 \mathrm{~nm} \pm 4 \mathrm{~nm}$. This method gives a narrower size distribution than any other method used. UV-Vis spectroscopy data for these nanoparticles is given in figures $4.10 \mathrm{a}$ and $4.10 \mathrm{~b}$.

\section{Absorbance spectrum for experiment 3}

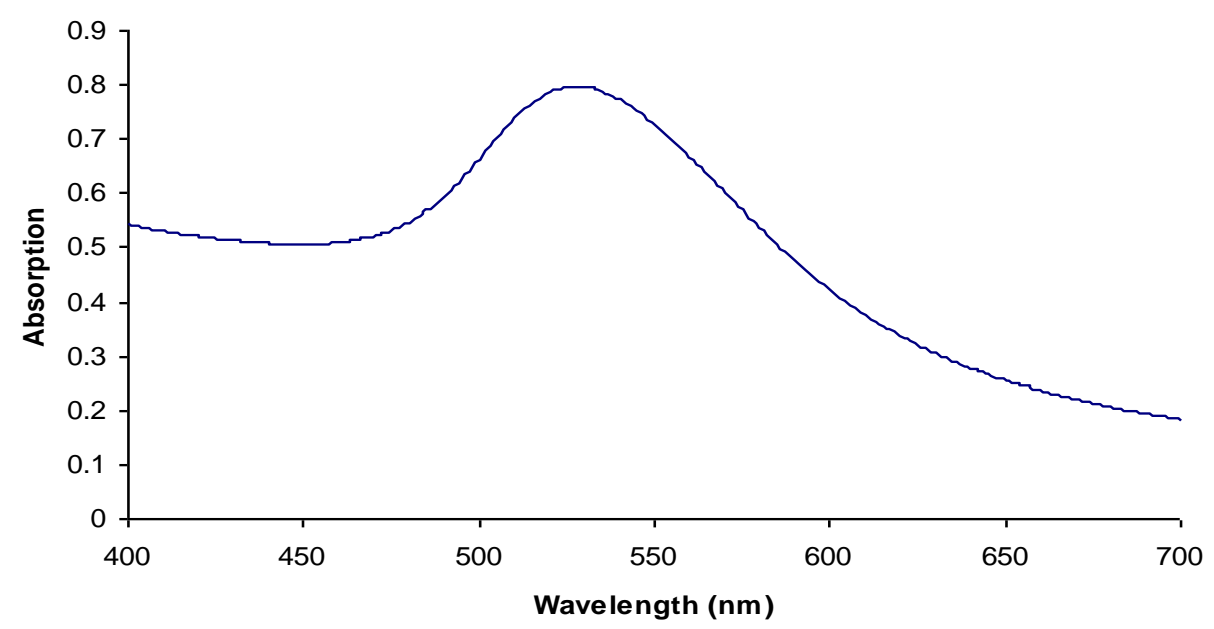

Figure 4.10(a): UV-Vis absorbance spectrum on the nanoparticles from experiment 3, which peaked at $532 \mathrm{~nm}$.

\section{Absorbance spectrum for experiment 4}

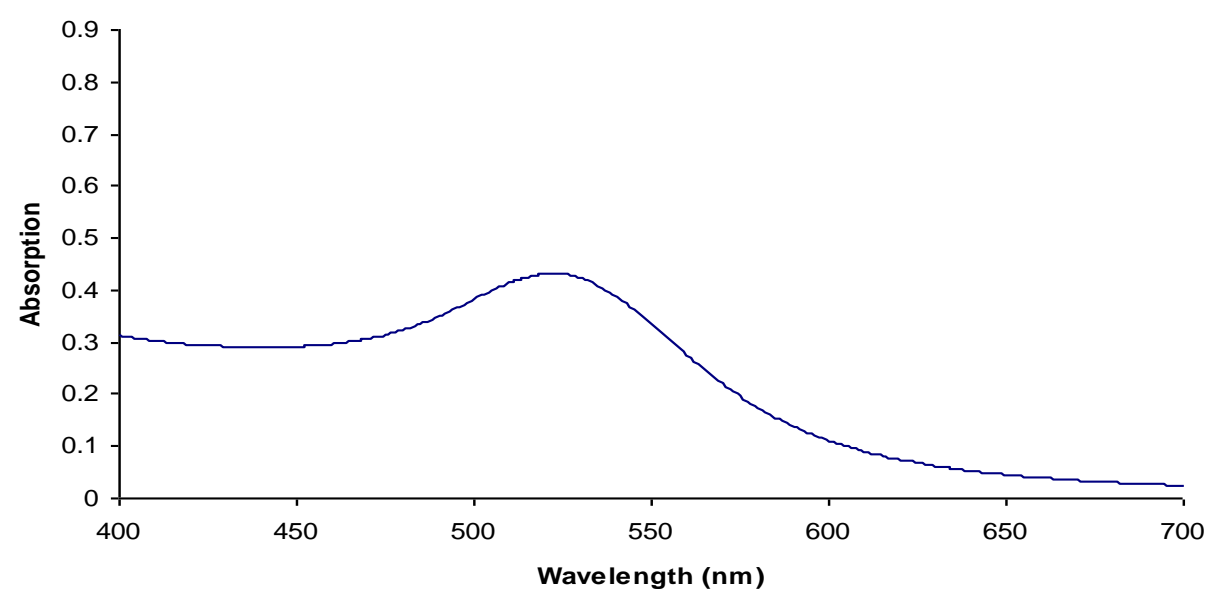

Figure 4.10 (b): UV-Vis absorbance spectrum on the nanoparticles from experiment 4 peaked at $523 \mathrm{~nm}$, which match literature expectations for gold nanoparticles with average diameter of $11 \mathrm{~nm}$.

As can be seen from figure 4.10, the absorbance spectrum peaks at a value of $532 \mathrm{~nm}$ for experiment 3 and $523 \mathrm{~nm}$ for experiment 4. The latter absorbance peak 
corresponds to the expected absorption value for gold nanoparticles with a diameter of $10 \mathrm{~nm} .[35,38,39,49,54]$

\section{Discussion}

Experiments using methods 3 and 4 prove that a better sample is produced in terms of shape and size distribution compared to the attempts made in experiments 1 and 2. A change in the concentration of citrate ions used, using a concentration ratio that is higher than the proposed 1:4 ratio of gold precursor to citrate ions, produces nanoparticles that are more evenly distributed and have the shape required for measurements on the qNano in this research. For this study, the shape of the gold nanoparticles produced is important, as signal profile generated by spherical nanoparticles is the only one studied in the research. There is no issue with having a different shape of nanoparticles but a change of shape will limit the ability to compare data with respect to data obtained from the polystyrene nanoparticles.

\subsubsection{Experiments 5(a) and 5(b)}

While this research was conducted, development of the qNano technology was also in progress. One of the upgrades for the technology involved its detection mechanism as well as the sensitivity of IZON's aperture. Measurements on the qNano require that the gold nanoparticles used should be larger than those produced. Therefore, samples from experiment 5 were made to match the technology available.

\section{$\underline{\text { Results }}$}

These gold nanoparticles were synthesized using Experiment 5 (refer table 4.1) using a method developed by Kimling J. et al. [42]. In this case, only the concentration of sodium citrate was significantly varied. Experiment 5(a) used $1.03 \mathrm{mM}$ of gold precursor and $1.43 \mathrm{mM}$ of sodium citrate compared to experiment $5(\mathrm{~b})$ which used $1.01 \mathrm{mM}$ of gold precursor and only $1.29 \mathrm{mM}$ of sodium citrate. The TEM images of the nanoparticles are given in figure 4.11. 


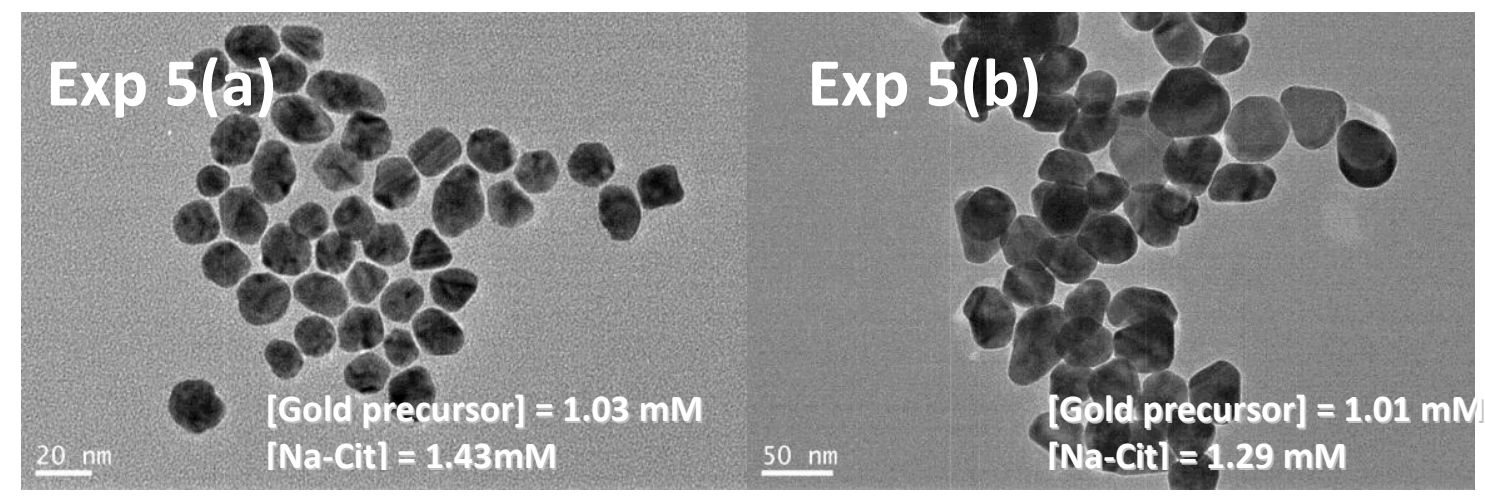

Figure 4.11: TEM images for experiment 5(a) and 5(b) gold nanoparticles. Sample from Experiment 5(b) shows some aggregation of particles that can be separated with sonication of the sample. Although both sample produced are not perfectly spherical, it both exist mostly as single particles, which is beneficial for measurement on the qNano instrument.

Figure 4.11 shows the two gold nanoparticle samples synthesized in the interest of making larger sized nanoparticles. It can be seen that experiment 5(a) gives gold nanoparticles that were more isolated than those synthesized with a lower concentration of sodium citrate. The nanoparticles in experiment 5(a) exist as single particles and not aggregates. Although experiment $5(\mathrm{a})$ produces a better sample in terms of dispersity of the nanoparticles, experiment $5(\mathrm{~b})$ has a larger diameter range that would work well with the qNano system. TEM images from experiment 5(b) however showed that the nanoparticles produced were multi-faceted. Figure 4.12 shows the size distribution of sample 5(a).

Size distribution of experiment $5(a)$

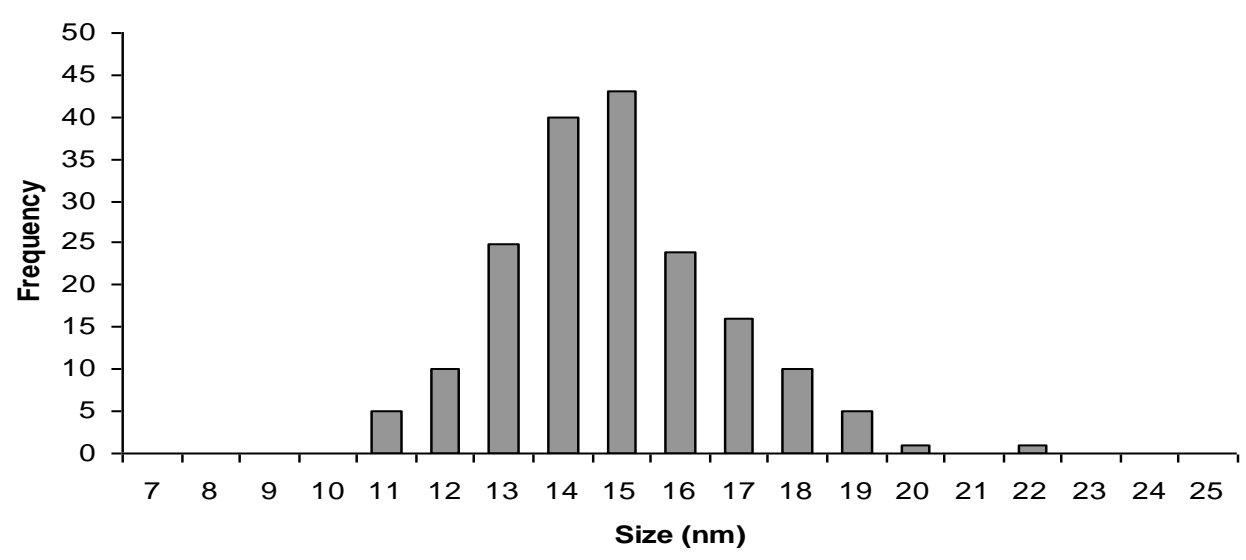

Figure 4.12: Size distribution of sample 5(a) shows that the average diameter of the sample is $15 \mathrm{~nm} \pm 5 \mathrm{~nm}$. 
Figure 4.12 gives the mean diameter of the sample from experiment $5(a)$ to be approximately $15 \mathrm{~nm} \pm 5 \mathrm{~nm}$. As with the previous samples from experiments 1 to 4, this is considered too small to work with the aperture. Size distribution of experiment 5(b) however was not obtained, as the nanoparticles in this sample are not largely spherical. Therefore, diameter comparison between experiments $5(a)$ and $5(b)$ cannot be made using the same histogram plot.

\section{Discussion}

Although experiment 5(a) produces samples that are more monodispersed, experiment $5(b)$ has much larger nanoparticles. Size is the more important parameter in the measurement at this stage, therefore samples from experiment $5(b)$ will be used regardless of the multi-faceted nanoparticles it produced as seen in figure 4.11. Synthesizing large sized nanoparticles proves to be challenging. This is because in order to have large sized gold nanoparticles, the concentration of the capping agent has to be considerably less than the desired concentration ratio between gold precursor and citrate ions. Decreasing the ratio produces larger sized nanoparticles but at the expense of the quality and shape of the nanoparticles. In addition, polydispersed gold nanoparticles will be obtained if the synthesized nanoparticles are larger than $30 \mathrm{~nm}$ [41].

\subsubsection{Synthesis conclusion}

Results from all the experiments (Experiments $1-5$ ) have shown that a more concentrated gold precursor results in smaller sized nanoparticles. Furthermore, the concentration of sodium citrate used affects the dispersity of the nanoparticles synthesized. [37]. Out of all the methods attempted, the method developed in experiments 3 and $4[46,49]$ produced the best quality samples to be used for measurements. Initial measurements will use samples from experiment $1(a)$ as the size of the nanoparticles (approximately $80 \mathrm{~nm}$ ) were suitable for measurements on the instrument taking into account the technology available at the time of measurement.

Later on, samples from experiment $5(\mathrm{~b})$ will also be used due to their larger particle diameter. The main reason many samples were synthesised is that the diameter and 
shape of the nanoparticles are crucial in the measurements on the qNano. Keeping one parameter constant such as particle shape would help in recognising signal output as it goes through the nanopore. Ensuring that synthesized gold nanoparticles have a diameter of more than $50 \mathrm{~nm}$ is also crucial at this stage as there is a limit to the detection capability of qNano. Therefore, maintaining the samples as close to IZON's standard polystyrene nanoparticles as possible would be very helpful in analysing data in the future.

\subsection{Measurement of gold nanoparticles}

Results in chapter 3 show that detection of polystyrene nanoparticles under electrophoresis is achievable. In this section, gold nanoparticles will also be run under the same conditions as the polystyrene nanoparticles. Therefore, polystyrene nanoparticles will be used as a reference for measuring gold nanoparticles. The gold nanoparticles' behaviour and response to the electric field and the constituents that make up the buffer solution will also be studied. The gold nanoparticles used were sourced using the citrate reduction synthesis method, $[37-64,78]$ as well as purchased from BB International - suppliers of reagents and particles for diagnostic use [92]. The synthesized gold nanoparticles have a diameter range of $10 \mathrm{~nm}$ to $15 \mathrm{~nm}$. This is typical of the diameter range for gold nanoparticles reduced by sodium citrate [41]. However, subsequent measurements on this instrument will use gold samples synthesized from experiment 1(a) and experiment 4 as the size of the nanoparticles are large enough to be detected by the instrument. Gold nanoparticles purchased from BB International were $100 \mathrm{~nm}$ in diameter.

Table 4.3 summarise the gold measurements that were done on the qNano instrument. 


\begin{tabular}{|c|c|c|}
\hline Section & Title & Parameters/Variables \\
\hline 4.3 .1 & Detection of gold nanoparticles & Voltage, aperture stretch \\
\hline 4.3 .2 & $\begin{array}{l}\text { Induced aggregation of gold } \\
\text { nanoparticles }\end{array}$ & $\begin{array}{l}\text { Buffer solution, concentration of } \\
\mathrm{KCl} \text { concentration of gold } \\
\text { nanoparticles }\end{array}$ \\
\hline 4.3 .3 & $\begin{array}{l}\text { Aggregation time of gold } \\
\text { nanoparticles }\end{array}$ & Measurement time \\
\hline 4.3 .4 & $\begin{array}{l}\text { Detection of gold nanoparticles with a } \\
\text { bandwidth of } 50 \mathrm{kHz}\end{array}$ & Voltage, aperture stretch \\
\hline 4.3 .5 & $\begin{array}{l}\text { Effect of concentration of gold } \\
\text { nanoparticles }\end{array}$ & Concentration of gold \\
\hline 4.3 .6 & $\begin{array}{l}\text { Indentifying two species of particles in } \\
\text { the same solution }\end{array}$ & Aperture stretch \\
\hline 4.4 & $\begin{array}{l}\text { Measurements of gold nanoparticles } \\
\text { with the Variable Pressure Module } \\
\text { (VPM) }\end{array}$ & $\begin{array}{l}\text { Pressure, voltage, concentration of } \\
\text { gold }\end{array}$ \\
\hline
\end{tabular}

Table 4.3: Summary of experiments for the measurement of gold nanoparticles.

\subsubsection{Detection of gold nanoparticles}

IZON's apertures have pores that are able to resolve particles down to $100 \mathrm{~nm} \pm 20$ $\mathrm{nm}$. This gives users limitations of the range of diameters that can be detected by the qNano, as particles smaller than this size range are drowned by the noise signal. Sample 1(a) of the synthesized gold nanoparticles was initially used as the sample has the largest diameter.

\subsubsection{Experimental Methods}

Sample 1(a) was dispersed in IZON's standard buffer solution in a 3:250 dilution i.e. $120 \mu \mathrm{l}$ of $1(\mathrm{a})$ in $10 \mathrm{ml}$ of buffer solution. The running conditions for gold nanoparticles were set at a voltage of $0.5 \mathrm{~V}$. This is a relatively high voltage compared to the one used in polystyrene $(0.3 \mathrm{~V})$. The choice of voltage used is because of the small diameter of the nanoparticles and is a trade off between the particle diameter and the background 
electrical noise it generated. Surface charge for smaller diameter nanoparticles is less than those with a larger diameter.

Particle translocation works via electrophoresis, so a smaller surface charge means the need for a bigger driving force to push the particle through the aperture. Therefore, a higher voltage bias was used in this measurement. The higher voltage bias used also means that the more ionic carrier goes through the pore as well. This generates a lot of background noise in the live data trace, which might drown in the background current trace. For the qNano, an aperture stretch of $\Delta \mathrm{XY}=14.8 \mathrm{~mm}$ was used, which corresponds to a baseline current of approximately $102 \mathrm{nA}$. All measurements were taken at 10 minutes duration.

\subsubsection{Results and Analysis}

Figure 4.13 where screenshots of blockade events as detected by the instrument were displayed.

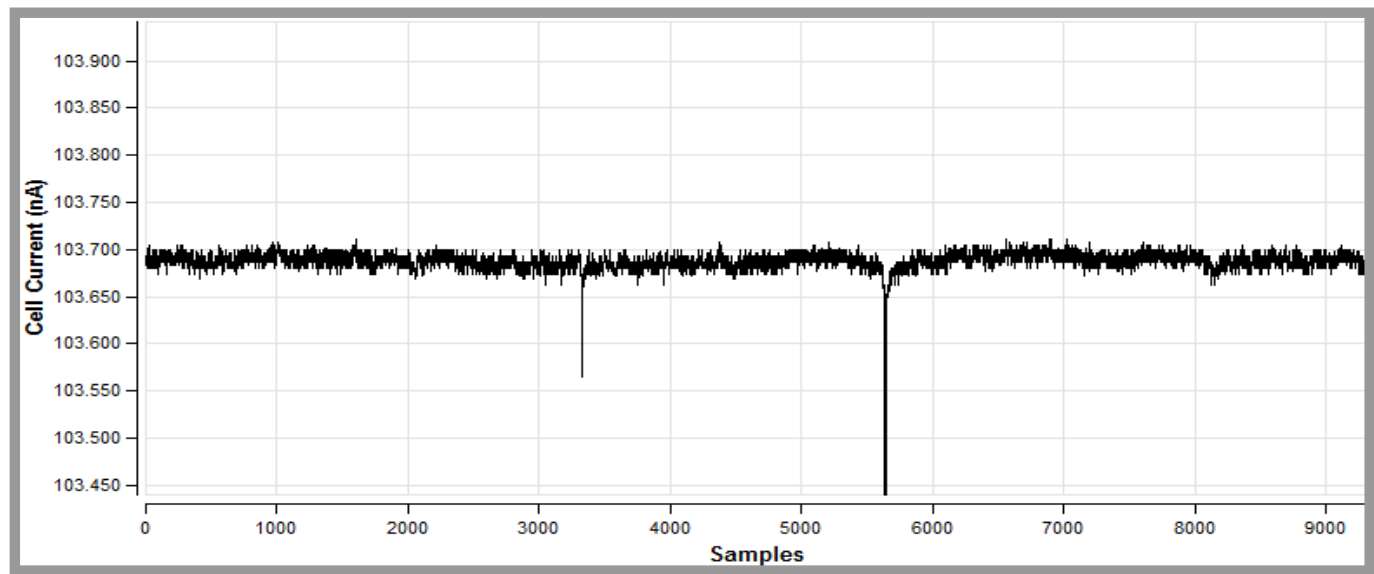

Figure 4.13: Screenshot of blockade event detected for gold nanoparticles. Very low translocation of gold nanoparticles was observed in this measurement as can be seen in the screenshot.

Figure 4.13 show the screen shot of the translocated gold nanoparticles as detected by the instrument, given by the downward peaks. In figure 4.13 , it appears that the depth of the blockade events detected the presence of two sizes of gold nanoparticles. The depth of the blockade events gives information on the diameter of particles as the electrical resistance has a cubic relationship to the radius of the nanoparticles. 
Therefore, as shown in figure 4.13, two different blockade depth is observed which means two diameters of gold nanoparticles are present in the solution. The frequency of nanoparticle translocation is very low even at a running voltage of $0.5 \mathrm{~V}$, as compared to the polystyrene samples shown in the previous chapter. The distribution of detected gold nanoparticles is given in figure 4.14 .

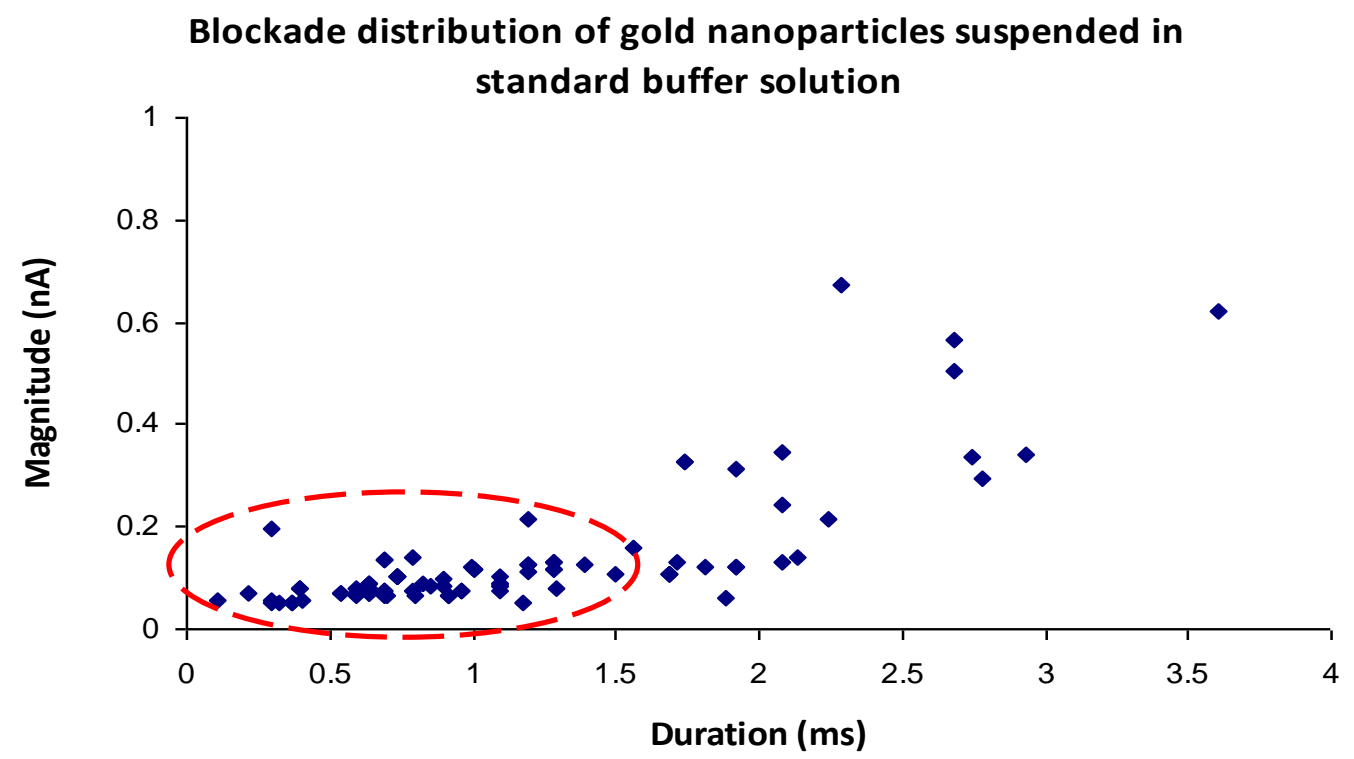

Figure 4.14: Distribution of gold nanoparticles translocating through the nanopore. Compared to the scatter plot of polystyrene particles in previous chapters, it was found that the frequency of particle translocation is much lower.

Figure 4.14 shows the distribution of gold nanoparticles as a function of magnitude. It would appear that most of the data points concentrate at a region denoted by the red bubble in figure 4.14, at a magnitude less than $0.2 \mathrm{nA}$ and with a translocated duration of less than $2 \mathrm{~ms}$. This distribution demonstrates that most of the nanoparticles move through the pore with approximately the same duration and magnitude. As IZON'S aperture can only detect down to $100 \mathrm{~nm}$ nanoparticles, blockade events that lie outside the red bubble are assumed to be larger gold aggregates. Translating the $\mathrm{Y}$-axis of figure 4.14 into a histogram distribution will clarify this point further. This is shown in figure 4.15 . 


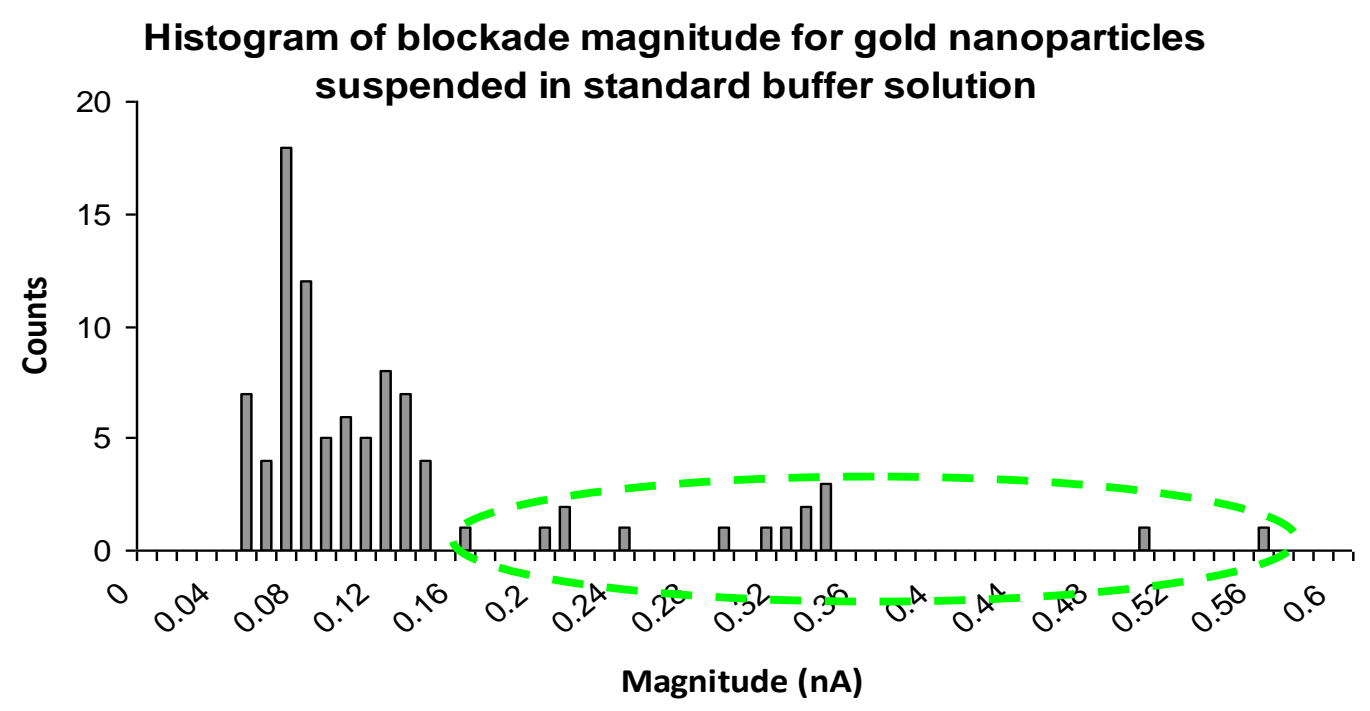

Figure 4.15: A histogram of the blockade magnitude of gold nanoparticles going through the nanopore. Data points in the green bubble indicate that there are larger sized gold nanoparticles present in the solution, which could indicate the presence of gold aggregates.

In figure 4.15 , most of the data points range between blockade magnitudes of 0.05 0.15. The green bubble shows the data points corresponding to the larger blockade magnitudes that deviate from the red bubble shown in figure 4.14. This suggests that larger species are present in the solution and these are assumed to be aggregates of gold nanoparticles.

\subsubsection{Discussion}

One hypothesis is that the presence of potassium chloride $(\mathrm{KCl})$ in the buffer causes the gold nanoparticles to aggregate. The stability of metal nanoparticles has been extensively studied and can be explained by the Derjaguin-Landau-Verwey-Overbeek (DLVO) theory. DLVO theory states that colloids or nanoparticles in a solution interact with each other via an attractive Van Der Walls force [62]. The strong attractive force between the nanoparticles is minimised due to the electrostatic and/or steric repulsive force between charged conjugates present in the aqueous media. This electrostatic repulsion comes from the electrical double layer; a layer that consists of the capping agent of the gold nanoparticles, and the layer of counterions (called the diffuse layer). The thickness of this diffuse layer is inversely proportional to the square root of the 
electrolyte concentration $[60,62]$. This means that any charged nanoparticles placed in an electrolyte cause a change in the ionic balance and this will attract the counter ions to form the double layer.

The thickness of this double layer depends on the concentration of the electrolyte. A concentrated electrolyte will increase the ionic strength and compress the double layer. When the thickness of the double layer becomes too small it screens the electrostatic repulsion between the nanoparticles and hence, induces a permanent aggregation [41, 49, 52, 55,57,58,59, 64]. Levy et al. [44] shows that on addition of sodium chloride $(\mathrm{NaCl})$, the gold nanoparticles synthesized via the citrate reduction method aggregates. Liu and Guo [50] reported that citrate stabilized nanoparticles have a poor electrostatic ability even if a minimum of $10 \mathrm{mM}$ of salt is present. The usual method of tracking aggregation is via UV-Vis absorption [48]. In one study, Wang and Sun [59] reported a peak of between $700 \mathrm{~nm}$ and $800 \mathrm{~nm}$ with a concentration of $\mathrm{KCl}$ of more than $0.38 \mathrm{M}$.

To solve the aggregation issue, various methods of stabilization of gold nanoparticles have been investigated [44, 49-53]. One of which is via modifying the gold nanoparticles' surface functionality. Levy et al., [44] used pentapeptide CALNN - where $\mathrm{C}$ is the carbon backbone; $\mathrm{A}$ is the alanine; $\mathrm{L}$ is the leucine; and $\mathrm{N}$ is the asparagine group - to prevent aggregation of gold nanoparticles in the presence of salt ions. Adsorbing the peptide layer to the surface of the gold nanoparticles hinders electrolyte-induced aggregation. This is possible as the positively charged amino group on the peptide is electrostatically attracted to the negatively charged surface of the gold nanoparticles. Sugunan et al., [49] on the other hand used chitosan - derived from chitin - to stabilize the gold nanoparticles in the presence of salt ions to prevent aggregation. The stabilization of gold nanoparticles using organic layers $[44,49,60]$ to work with IZON's buffer system is a possible study that can be further investigated in the future.

Gold nanoparticles exhibit blockade counts that are approximately $10 \%$ of the blockade counts for polystyrene particles. The reason for this observation is possibly 
due to a lower concentration of the gold nanoparticles used (in terms of particles $/ \mathrm{ml}$ ) in the sample. This decrease in concentration is more prominent especially if individual particles aggregate in the buffer system, which reduces the availability of nanoparticles to translocate through the nanopore. Another possible explanation is due to the size limitation of nanopore to allow the gold aggregates to pass through, i.e. the nanopore is smaller than the aggregated gold nanoparticles.

Ji et al., [39] conducted a study that explored the effect of $\mathrm{pH}$ solution to the zeta potential of gold nanoparticles. It was found that the value of zeta potential increases as the $\mathrm{pH}$ of the solution increases $[62,63]$. Ji found that aggregation occurs instantaneously at low $\mathrm{pH}$ range $[39,62]$. The low $\mathrm{pH}$ range causes a lowering of the potential barrier between nanoparticles in the solution. The $\mathrm{pH}$ of the buffer solution used was 8 , and therefore this reasoning is not the cause of nanoparticle aggregation and is discounted.

\subsubsection{Induced aggregation of gold nanoparticles}

Results from section 4.3.1 show that the blockade signals detected are not of single nanoparticles. Gold aggregates formed in the electrolyte are most likely due to the presence of $\mathrm{KCl}$ salt. In this section, gold nanoparticles was suspended in sodium citrate (Na-Cit) buffer, the same solution that was used in making the gold nanoparticles and $\mathrm{KCl}$ will be added in situ while the measurement is being taken. This part of the study focuses on determining if the salt present in IZON's buffer solution is the cause of particle aggregation.

\subsubsection{Experimental Methods}

$\mathrm{Na}$-Cit buffer solution (0.5 M) was prepared by dissolving $73.525 \mathrm{~g}$ of $\mathrm{Na}$-Cit in $500 \mathrm{ml}$ of deionised water. The $\mathrm{pH}$ of the solution was adjusted to 6.3 by adding hydrochloric acid $(\mathrm{HCl})$ to the stock solution. $0.05 \mathrm{M}$ of $\mathrm{Na}$-Cit buffer was then prepared by taking 4 $\mathrm{ml}$ of the stock solution and then diluting with $36 \mathrm{ml}$ deionised water. The final $\mathrm{pH}$ for this solution is 6.13 . To make the $\mathrm{KCl}$ salt solution of concentration $0.5 \mathrm{M}, 0.1835 \mathrm{~g}$ of $\mathrm{KCl}$ pellets were dissolved in $5 \mathrm{ml}$ of deionised water. 
Prior to the gold nanoparticle measurement, all apertures used in this section were initially tested with IZON's standard buffer to ensure that the pore opens as expected. Once the pore is opened, the system is replaced with Na-Cit buffer and the current reading was allowed to stabilize. Polystyrene nanoparticles with diameter of $100 \mathrm{~nm}$ and $70 \mathrm{~nm}$ were used to find the suitable stretch range of the aperture. These nanoparticles were suspended in Na-Cit in a 1:100 dilution.

Gold nanoparticles that were used in this section are of sample 4. These nanoparticles were suspended in $\mathrm{Na}$-Cit buffer and were introduced to the upper fluid cell at two different dilutions, 1:100 and 1:50 dilution. The aperture was stretched to a maximum of $\Delta X Y=9.35 \mathrm{~mm}$ and closed down to $\Delta X Y=3 \mathrm{~mm}$. To induce aggregation of gold nanoparticles, $10 \mu \mathrm{l}$ of $0.5 \mathrm{M} \mathrm{KCl}$ was added to the top fluid cell to confirm that aggregates of gold nanoparticles will be formed upon addition of the $\mathrm{KCl}$ salt. The amount of $\mathrm{KCl}$ added was varied but this parameter was not investigated extensively. $\mathrm{KCl}$ was in relation to the amount of gold nanoparticles in the upper fluid cell and trials of $1: 1,1: 5$, and $1: 10$ ratios of gold: $\mathrm{KCl}$ was employed to see the effect of the concentration of $\mathrm{KCl}$ as a substance to induce aggregation.

\subsubsection{Results and Analysis}

Polystyrene nanoparticles suspended in $\mathrm{Na}$-Cit give promising blockade counts. However, the same cannot be said for gold nanoparticles even with the addition of $\mathrm{KCl}$ salt in excess in the space of the measurement time. No blockades were detected with just gold nanoparticles suspended in the citrate buffer, which was expected, as the nanoparticles are too small to be detected by the instrument. On the other hand, addition of $\mathrm{KCl}$ salt to the upper fluid cell also did not produce any blockade events, which suggest that gold aggregates did not form within the measurement time.

\subsubsection{Discussion}

It has been stated earlier that gold nanoparticles used in this section are those from sample 4 with a mean diameter of $11 \mathrm{~nm}$. The gold nanoparticles was suspended in a citrate buffer, and therefore no aggregations occurred as citrate ion serve as the stabilising agent. This would mean that the gold nanoparticles exist in the solution as 
single nanoparticles. Furthermore, with this limitation of particles size, it is expected that no blockades would be detected because the aperture is not sensitive enough to detect nanoparticles with that small diameter.

The addition of $\mathrm{KCl}$ salt to gold nanoparticles suspended in $\mathrm{Na}$-Cit buffer was added to induce and confirm that gold nanoparticles aggregate in the standard buffer solution. However, no observation of gold blockades was detected. This would suggest that the time taken for the gold nanoparticles to aggregate is not instantaneous and is longer than the time it takes to do the measurement. The time taken for gold aggregates to form would be an interesting study and this forms the basis for the next section. The longer time to observe this aggregation is also due to the low concentration (0.1 M) of $\mathrm{KCl}$ present in the buffer solution.

Wang and Sun [59] reported that to induce aggregation of gold nanoparticles, a concentrated solution of $5 \mathrm{M} \mathrm{KCl}$ and $\mathrm{NaCl}$ was needed. The ratio of gold nanoparticles to salt solution has a maximum ratio of $1: 4$. With this concentrated solution, the aggregation occurs instantaneously. In another study by Yang et al. [54], cetyltrimethylammonium bromide (CTAB) was used to induce aggregation of citrate stabilized gold nanoparticles and it was found that $0.1 \mathrm{mM}$ of CTAB was enough to form gold aggregates. The absorbance peak at $585 \mathrm{~nm}$ supports this observation.

\subsubsection{Aggregation time of gold nanoparticles}

Following from results from the previous section, the aggregation time of gold nanoparticles in the buffer solution was investigated.

\subsubsection{Experimental Methods}

Sample 4 of gold nanoparticles was suspended in $5 \mathrm{ml}$ of $0.34 \mathrm{M} \mathrm{Na-Cit} \mathrm{in} \mathrm{a} \mathrm{1:100}$ dilution. IZON's standard polystyrene nanoparticles with a diameter of $70 \mathrm{~nm}$ were also suspended in the citrate buffer in a 1:500 dilution. The polystyrene nanoparticles were used as a reference and have dual purpose; to find the right stretch range as well as a reference on the size of gold aggregates. 
Once a suitable aperture stretch was found, the fluid cells were refreshed and gold nanoparticles were measured. $0.5 \mathrm{M}$ of $\mathrm{KCl}$ salt was added to the upper fluid cell and measurements were taken every 30 minutes. The running conditions for this are set to a running voltage of $0.3 \mathrm{~V}$ for the polystyrene nanoparticles and $0.28 \mathrm{~V}$ for the gold nanoparticles. The magnitude threshold - the blockade magnitude's values at which the instrument will record detected blockade events - are set to a value of $[0.08,2] \mathrm{nA}$ for polystyrene and $[0.125,2] \mathrm{nA}$ for the gold nanoparticles. The notation of $[x, y] n A$ denotes the lower and upper magnitude threshold that will be recorded in the software.

\subsubsection{Results and Analysis}

There were no blockade events detected in the first two hours of the experiment running. However, particle blockades began to be detected in the third hour. The distribution of polystyrene and gold nanoparticle aggregates is given in figure 4.16.

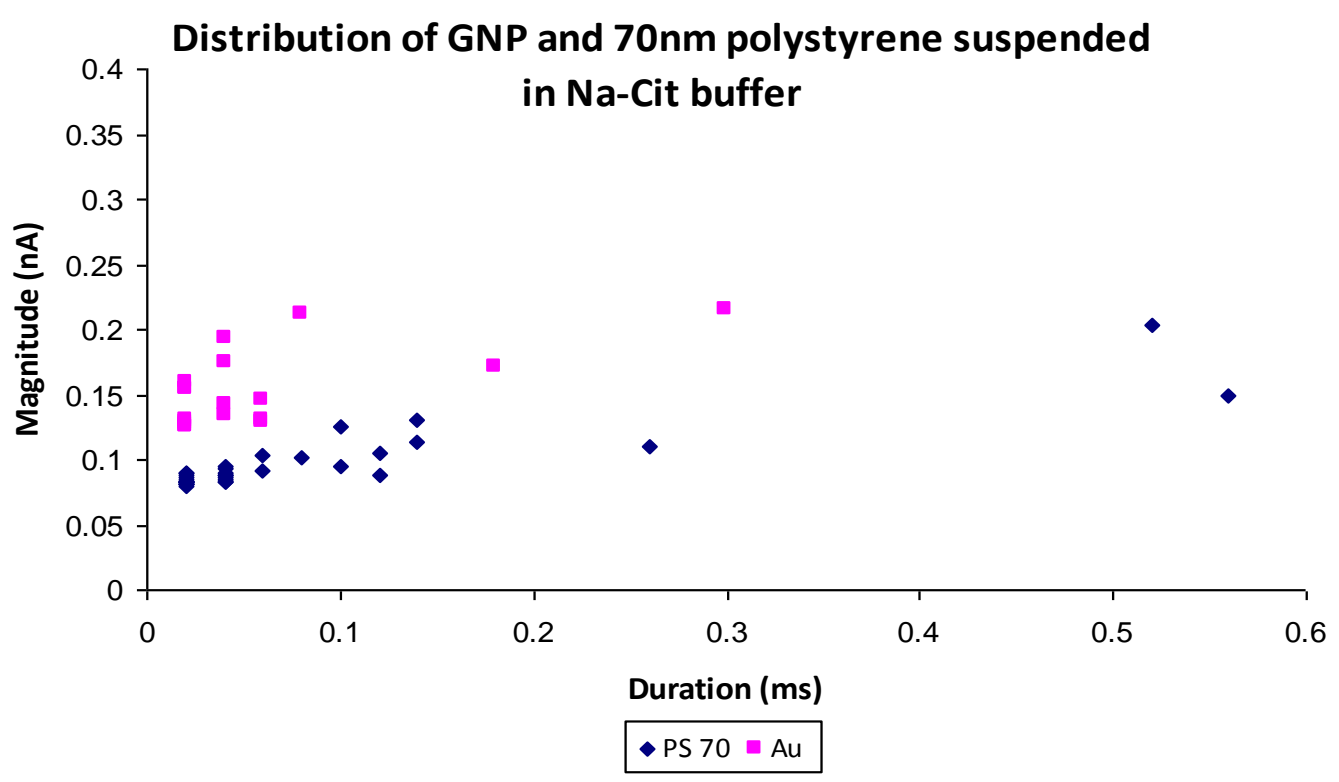

Figure 4.16: Distribution of $70 \mathrm{~nm}$ IZON's polystyrene nanoparticles and $11 \mathrm{~nm}$ gold nanoparticles suspended in Na-Cit buffer. The scatter plot shows that the size of the gold particles are larger than the $70 \mathrm{~nm}$ particles based on the size of the blockade magnitude which suggest that aggregation of gold nanoparticles in IZON's buffer solution occurs after three hours. 
Figure 4.16 shows that the gold nanoparticles aggregate in the citrate buffer upon the addition of the $\mathrm{KCl}$ salt. After three hours of reacting, the gold aggregates formed are larger than the $70 \mathrm{~nm}$ nanoparticles. This is shown in figure 4.16 by the larger blockade magnitudes of gold as compared to the signals from the $70 \mathrm{~nm}$ blockades. As suspected, the aggregates did not occur spontaneously. The rate of formation of gold aggregates most likely depends on the concentration of the $\mathrm{KCl}$ salt used relative to the concentration of the gold nanoparticles available in the solution.

\subsubsection{Discussion}

The aggregates detected seem to be noticeably larger than the $70 \mathrm{~nm}$ nanoparticles. Data obtained from this measurement seems to suggest that only uniform-sized aggregates are formed. However, it has to be noted that the magnitude threshold used for the gold measurement is much higher than the ones used for polystyrene as the citrate buffer produces a much higher background noise. This is partly why smaller aggregates of gold nanoparticles in the solution were not detected, as they can be drowned in the noise signal.

Wang and Sun [59] studied the stability of gold nanoparticles with respect with time in the presence of $0.5 \mathrm{M} \mathrm{KCl}$ salt. In their study, the extent of aggregation was observed using UV-Vis spectroscopy every 20 minutes for a period of three hours. It was found that the intensity of the absorbance peak decreases and a red shift of the UV-Vis absorbance peak were observed for $5 \mathrm{~nm}$ gold nanoparticles in the presence of $\mathrm{KCl}$ salt. At the end of the three hour period, the absorbance peak nearly completely disappeared which suggests that aggregation of the gold nanoparticles occurred. This was substantiated by the precipitate seen by the naked eye $[59,63]$.

\subsubsection{Detection of gold nanoparticles with a bandwidth of $50 \mathrm{kHz}$}

Part of the problem in detecting gold nanoparticles suspended in standard buffer solution is due to the limitation of the instrument itself. By this stage of study, IZON had upgraded its technology so that it has a faster rate of detection signal, from a bandwidth of $10 \mathrm{kHz}$ to $50 \mathrm{kHz}$. This increase in bandwidth means that there is more detection signals generated by the instrument per unit time and therefore; smaller 
nanoparticles are able to be accurately detected [13]. This part of study aims to see translocation of $11 \mathrm{~nm}$ gold nanoparticles suspended in the standard buffer solution using apertures that can resolve $100 \mathrm{~nm}$ polystyrene nanoparticles. Suspending the gold nanoparticles in IZON's buffer solution also means that translocation detected is of gold aggregates and not of single nanoparticles.

\subsubsection{Experimental Methods}

Trial runs using $100 \mathrm{~nm}$ standard polystyrene nanoparticles were used to calibrate the aperture. These nanoparticles were suspended in standard buffer solution in a 1:250 dilution. Samples of gold nanoparticles were prepared by adding $180 \mu \mathrm{l}$ of $0.37 \mathrm{M}$ of sample 4 gold nanoparticles to the buffer solution giving the dilution of gold nanoparticles in the buffer solution to be 1:14. This gives a concentration of nanoparticles in the buffer to be $1.61 \times 10^{19}$ particles $/ \mathrm{ml}$.

In this section, the running voltage used was set at $0.3 \mathrm{~V}$ throughout and the threshold for magnitude blockade was set at $[0.035,1] \mathrm{nA}$ or $[0.04,1] \mathrm{nA}$ depending on the noise level. Again, the notation $[x, y] n A$ represents the lower limit and upper limit of the magnitude threshold respectively.

\subsubsection{Results and Analysis}

IZON's standard $100 \mathrm{~nm}$ nanoparticles were used to calibrate the aperture. As stated before, this is the first attempt at detecting metal nanoparticles therefore the signal trend is unknown and polystyrene nanoparticles were used as a reference. These polystyrene nanoparticles were suspended in a 1:250 dilution with a setting of $0.3 \mathrm{~V}$ for its running voltage and a working stretch at $\Delta X Y=6.75 \mathrm{~mm}$. A screenshot of this run is given in figure 4.17. 


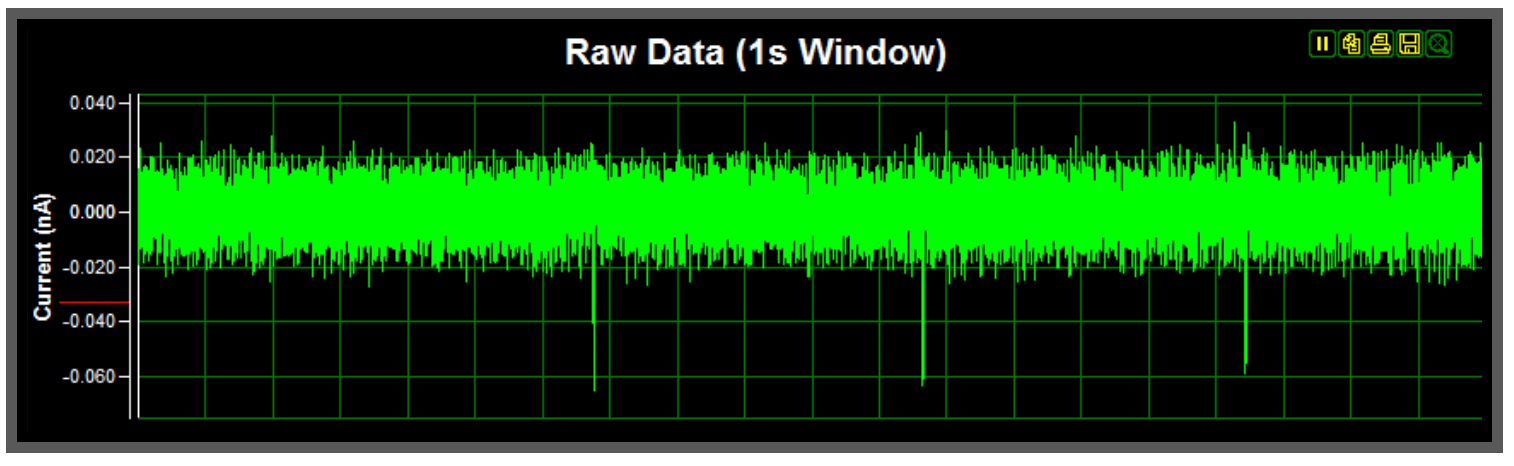

Figure 4.17: Screenshot of $100 \mathrm{~nm}$ polystyrene blockades. This is a screenshot of data in its 'Normalised Window'.

As seen in figure 4.17, a stretch of $\Delta \mathrm{XY}=6.75 \mathrm{~mm}$ gives a decent sized blockade magnitude for the $100 \mathrm{~nm}$ nanoparticles. Closing down the aperture to $\Delta \mathrm{XY}=4 \mathrm{~mm}$ will give a larger blockade magnitude. Therefore, measurements of gold nanoparticles suspended in the standard buffer solution were done at this stretch.

The gold nanoparticles used were introduced to the system with a dilution of 1:14. In theory, the gold nanoparticles cannot be seen because of their small diameter (11 nm). Therefore, there should not be any blockades observed when gold is introduced. However, there are blockades detected which means that the nanoparticles might be aggregating in the standard buffer solution as seen in figure 4.18 .

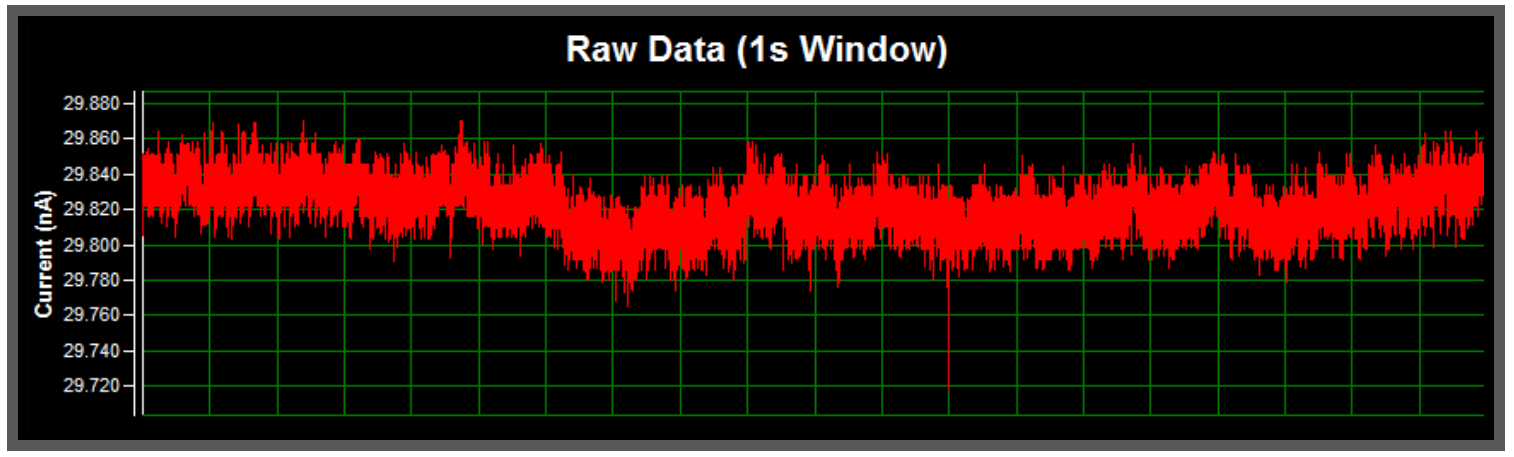

Figure 4.18: Screenshot of the signal with standard buffer solution and $11 \mathrm{~nm}$ gold nanoparticles in the upper fluid cell.

Figure 4.18 shows a blockade event of the gold nanoparticles. Again, as has been shown in section 4.3.1, the frequency of translocated nanoparticles is very low. Figure 3.18 also demonstrates that at this stretch, the signal of the current reading is quite noisy but resistive translocation can be seen nevertheless. 
Plotting a graph of blockade magnitude against duration, we can see how the gold nanoparticles in IZON's standard solution compare to the $100 \mathrm{~nm}$ nanoparticles in the same buffer solution. This is given in figure 4.19.

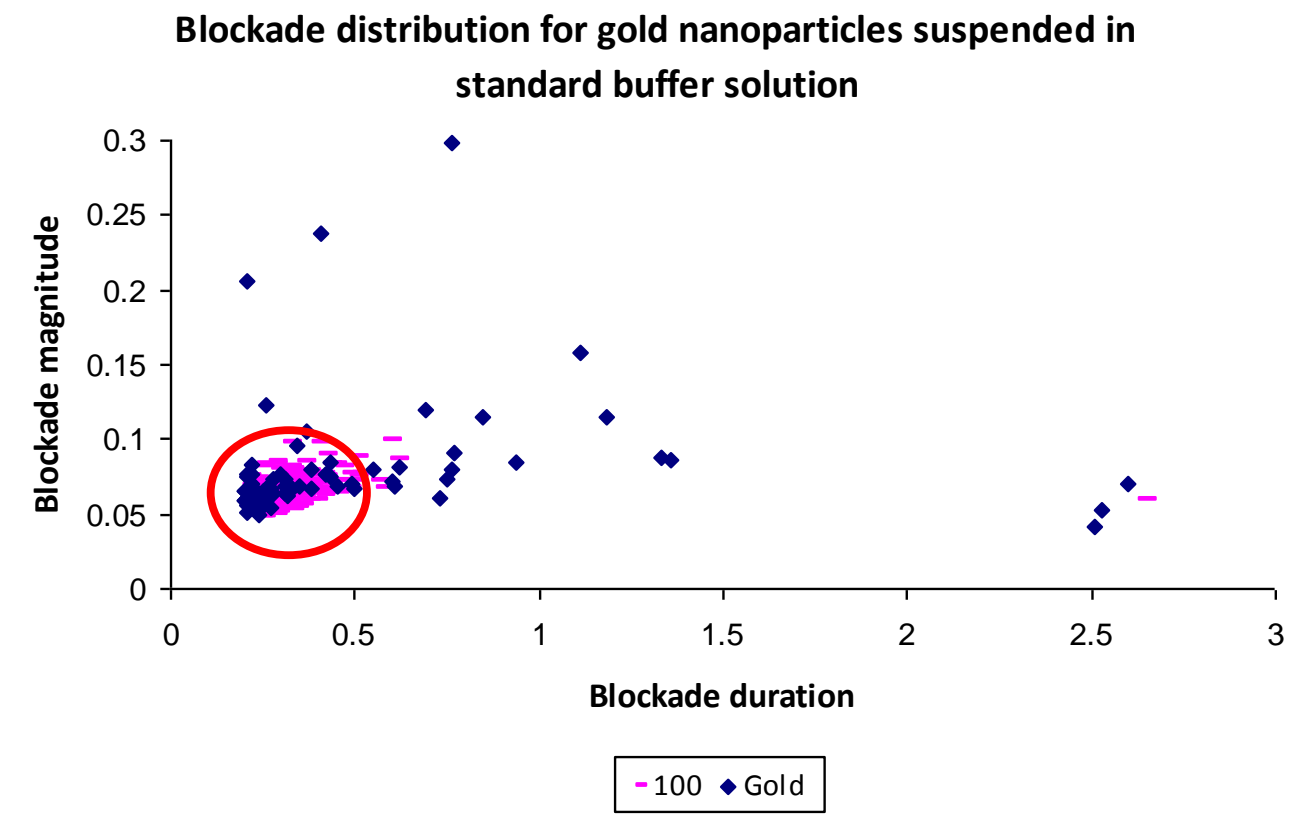

Figure 4.19: Distribution of both IZON's $100 \mathrm{~nm}$ polystyrene nanoparticles and 11 $n m$ gold nanoparticles in IZON's buffer solution. The red bubble in the figure shows that after some time, the gold nanoparticles suspended in the buffer solution have increased in size that approximates the $100 \mathrm{~nm}$ diameter polystyrene particles based on the overlapping of blockade data points.

As stated earlier, there should not be any blockades detected in this experiment, as the particle diameter used for the gold nanoparticles is approximately $11 \mathrm{~nm}$. However, figure 4.19 shows otherwise. The instrument detects blockade events for gold nanoparticles as they passes through the pore.

Through comparing the data points of $100 \mathrm{~nm}$ polystyrene nanoparticles and gold nanoparticles, it can be seen that the nanoparticles concentrate in the region denoted by the red bubble in figure 4.19. The bubble consist of a region of blockade magnitude between $0.03 \mathrm{nA}$ and $0.1 \mathrm{nA}$ and blockade duration between $0.03 \mathrm{~ms}$ and $0.5 \mathrm{~ms}$. Yet, for nanoparticles that are not supposed to be 'seen' by the instrument, the data points of the gold nanoparticles coincide with data of $100 \mathrm{~nm}$ polystyrene and spreads 
beyond the region of the red bubble. This indicates that the blockade events detected are not single particle events.

A point to note from figure 4.19 is that the polystyrene nanoparticles are monodisperse as the data points concentrates regularly within the region of the red bubble. Gold nanoparticles on the other hand show a random scatter showing a wide range of size distribution, which suggests that gold nanoparticles capped with citrate buffer form aggregates in IZON's standard buffer solution.

\subsubsection{Discussion}

As figure 4.19 shows, blockades of gold nanoparticles are present when they are not expected. Signals from the gold nanoparticles produced a large blockade magnitude, which imply that large gold aggregates were present in the system. Also, because 100 $\mathrm{nm}$ nanoparticles were seen at an aperture stretch of approximately $\Delta \mathrm{XY}=6 \mathrm{~mm}, \mathrm{a}$ decrease of aperture stretch to $\Delta \mathrm{XY}=4 \mathrm{~mm}$ would not decrease the pore size down enough for it to detect $11 \mathrm{~nm}$ nanoparticles. Therefore, it is assumed that the signal seen is the translocation of aggregated gold nanoparticles that is formed due to the presence of $\mathrm{KCl}$ salt in the standard buffer solution.

In some instances in the raw data window, there were upwards conductive pulses observed as showed by the screenshot in figure 4.20 .

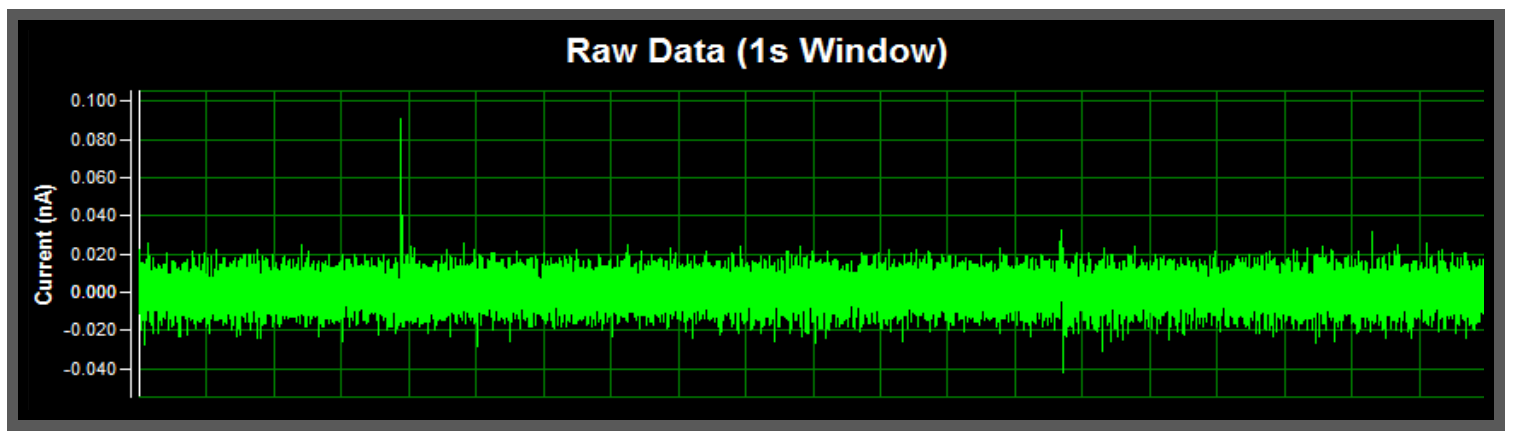

Figure 4.20: Screenshot of the normalised signal with standard buffer solution and 11 $n m$ gold nanoparticles shows an upward spike (conductive pulses) recorded by the instrument. 
An upward spike in figure 4.20 possibly means a conductive trace from the aggregated gold nanoparticles. Conductive spikes are observed because there is a higher ionic density where the gold nanoparticles translocate through the pore. When nanoparticles pass through the pore, there is $\mathrm{X}$ amount of volume displaced by it. Depending on the surface charge of the particle, the amount of ionic density around the nanoparticles will change accordingly. This corresponds to a decrease or increase of ionic density. A decrease in ionic density corresponds to a downward spike and vice versa. From this figure, it can be seen that there is an upward spike meaning that there is an increase of charge density in the detection area. A possible explanation of this result is a higher surface charge on the gold aggregates. However, no evidence can yet be supplied to substantiate this hypothesis.

The measurement in this section was done with an upgraded version of the technology from a $10 \mathrm{kHz}$ bandwidth to $50 \mathrm{kHz}$. A few problems were encountered while running the instrument. One of them is the difficulty in getting a current running when the upper fluid cell is taken to be cleaned. This could be due to the aperture being blocked by the testing nanoparticles prior to being shipped. Another issue is the difficulty to differentiate signals due to the upgraded software. The new software has an autozoom mode of the current baseline by default. Untrained users may find this confusing, as it was difficult to determine whether the blockades detected were actual single blockade events or a higher noise in the baseline current.

\subsubsection{Effect of concentration of gold nanoparticles}

The aim in this section is to investigate the relationship between concentrations of gold nanoparticles suspended in the standard buffer solution. As is reported in previous sections in this chapter, gold nanoparticles synthesized via the citrate reduction method aggregate in the IZON standard buffer solution due to the presence of $\mathrm{KCl}$. This section studies the effect of varying the dilution of gold nanoparticles in the buffer system and how it changes i.e. either producing larger aggregates or small aggregates with higher number of nanoparticles. 


\subsubsection{Experimental Methods}

The gold nanoparticles used were from sample 4 and they were suspended in the standard buffer solution in a 1:1000, 1:100, and 1:10 dilution. The measurement was done in increasing concentration so as not to block the nanopore and therefore gives a false blockade reading. Running conditions were set with a working aperture stretch $(\Delta \mathrm{XY})$ window between $9.79 \mathrm{~mm}$ and $10.07 \mathrm{~mm}$. The workable running voltage for this measurement was found to be at $0.2 \mathrm{~V}$ and the blockade threshold was set at $[0.05,1]$ nA for measurements of $A u 1: 10$, and $[0.04,1] \mathrm{nA}$ for both 1:100 and 1:1000 dilution. Each measurement was taken for a time of 1 minute.

\subsubsection{Results and Analysis}

Results from sections 4.3.1, 4.3.2, and 4.3.3 show that the gold nanoparticles produce a resistive blockade event. Figure 4.21 shows the distribution of gold nanoparticles suspended in the standard buffer solution at different concentrations.

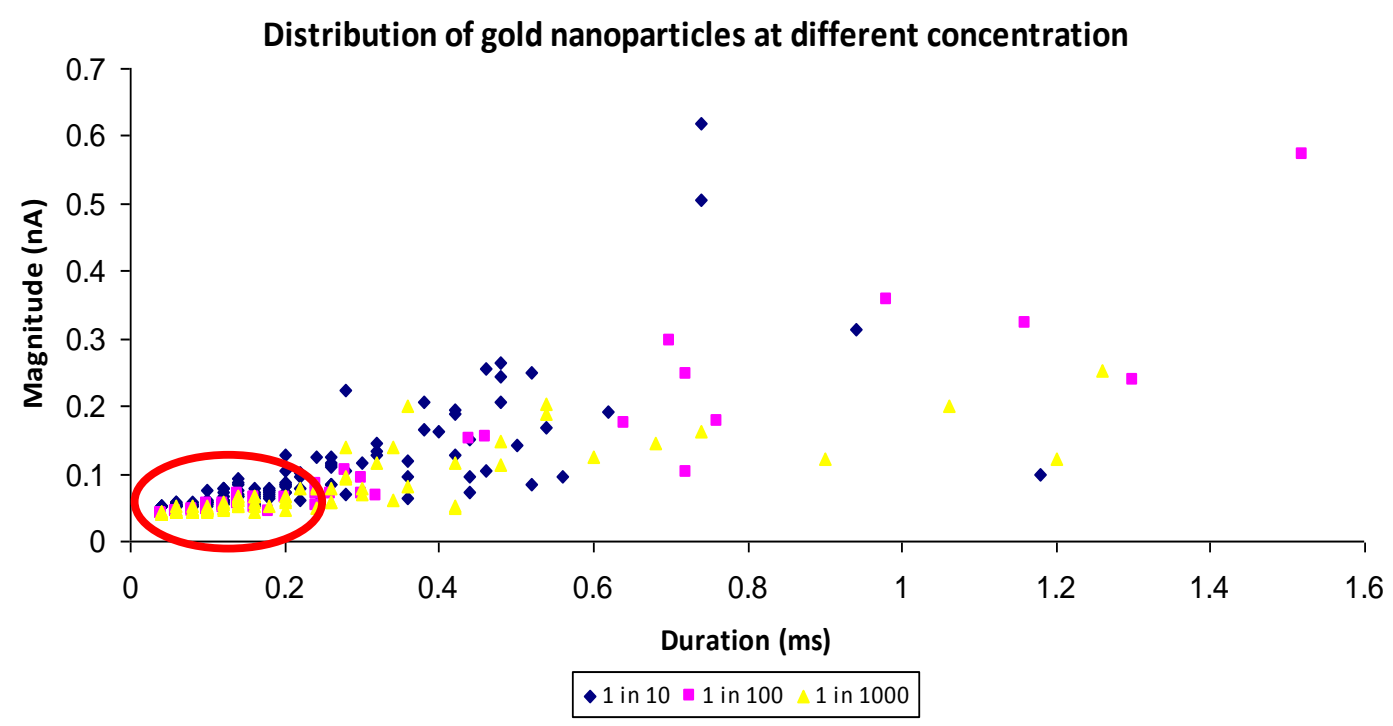

Figure 4.21: Scatter plot of gold nanoparticles dispersed in the standard buffer solution at different concentration. Data points outside the region of the red bubble seems to indicate that the quantity of gold nanoparticles aggregates increases as the concentration increases.

In figure 4.21, blockade events with bigger magnitudes are observed as the particle concentration is raised. It can be seen that most blockade events are detected within 
the region of the red bubble (in figure 4.21) i.e. under $0.2 \mathrm{~ms}$ of translocation duration and a maximum of $0.1 \mathrm{nA}$. However, data points detected beyond this red bubble confirm the presence of bigger nanoparticles that are suspected to be aggregates of gold nanoparticles in the buffer solution. The effect of concentration to the particle diameter can be shown in figure 4.22 .

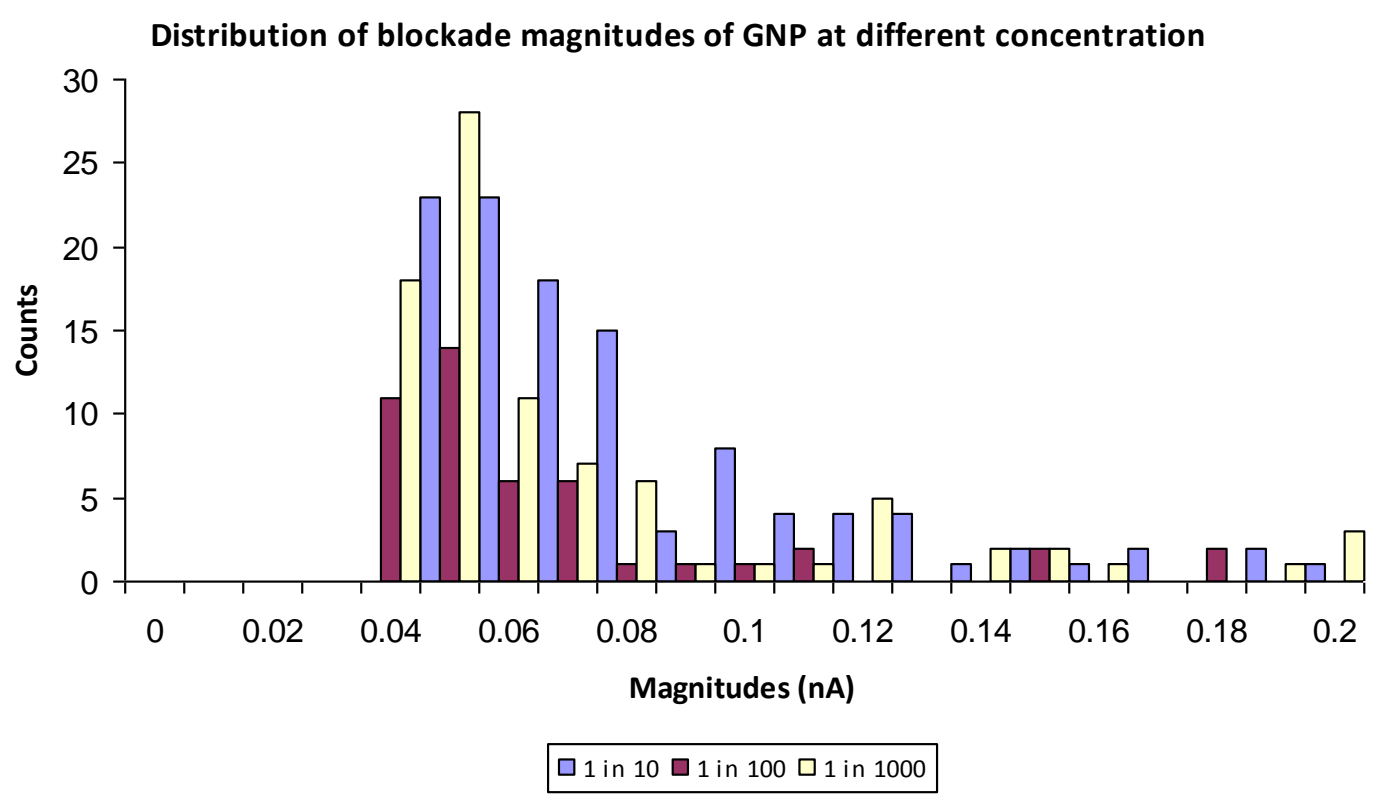

Figure 4.22: A histogram of blockade magnitudes of gold nanoparticles dispersed in standard buffer solution at different concentration. The threshold was set at 0.04 $n A$ therefore only blockades that have a magnitude larger than this value will be recorded.

Figure 4.22 shows a distribution of blockade events as the concentration of gold nanoparticles suspended is increased. It is assumed that with the increasing concentration, the presence of larger sized nanoparticles in the solution is widespread. A monodisperse sample would produce a narrow-spread distribution. Figure 4.22 show that nanoparticles that are suspended in the lowest concentration give a tighter distribution as compared to the two other concentrations. Therefore, the results suggest that as the concentration gets higher, the size of particle diameter in the solutions gets larger hence the broader distribution. 


\subsubsection{Discussion}

The concentration of gold nanoparticles suspended in IZON's standard buffer solution also plays a role in determining the final size of gold aggregates. As seen in figure 4.22, the more concentrated dilution produces larger nanoparticles in higher quantity as represented by the amount of larger magnitude blockade events beyond the red bubble region. This suggests that the size of the aggregates depends on the amount of gold available in the solution. The final diameter of aggregated gold nanoparticles depends also on the length of time it suspends in the standard buffer solution.

The mechanism of gold nanoparticles' aggregation in IZON's standard buffer solution possibly follows the same route for seeded growth of gold nanoparticles but this hypothesis is yet to be established. Njoki et al. [96] studied the aggregative mechanism of seeded growth of monodisperse gold nanoparticles. Critical aggregates will form in the induction stage, which will serve as an aggregation point in the aggregative growth mechanism. Free gold nanoparticles in the solution will coalesce with the critical aggregates until the point of depletion. The study reported that seeded growth gold nanoparticles have a polycrystalline structure due to the interparticle coalescence, which supports the aggregative growth model. However, the actual mechanism for gold aggregation in IZON's standard buffer solution is not yet understood at this stage of the research and further investigation could be conducted.

\subsubsection{Identification of two species of nanoparticles in a mixture: gold and polystyrene nanoparticles}

The detection of a mixture of polystyrene and gold nanoparticles has not been performed on this instrument. This section aims to study the ability of the instrument to resolve two species of nanoparticles. As established in chapter 3 - in regards to the bimodal distribution - the instrument has the potential to be better commercialized if it can demonstrate the capability of separating two different diameters of nanoparticles. Here, variation of two diameters and types of nanoparticles will be used to see if the instrument can distinguish each species - and diameters - of nanoparticles. IZON's standard nanoparticles of diameter $70 \mathrm{~nm}$ and gold 
nanoparticles purchased from BB International with diameter of $100 \mathrm{~nm}$ were used in this study.

\subsubsection{Experimental Methods}

All nanoparticles were suspended in the standard buffer solution in a 1:100 dilution. The $70 \mathrm{~nm}$ polystyrene nanoparticles were found to be best detected on a working stretch of $\Delta X Y=8.5 \mathrm{~mm}$ while the gold nanoparticles were found to be detected on a stretch of $\Delta X Y=12 \mathrm{~mm}$. However, the aperture stretch for the mixture of nanoparticles was found to be detected at a $\Delta X Y$ of $7.8 \mathrm{~mm}$. The running voltage used for all the measurements here is set to $\mathrm{V}=0.3 \mathrm{~V}$.

\subsubsection{Results and Analysis}

The results acquired from this measurement will be analysed in terms of blockade magnitude and nanoparticles' duration of translocation. The results of this is of interest as it is crucial to see if the instrument is able to distinguish between the two different diameters and types of nanoparticles present in the solution.

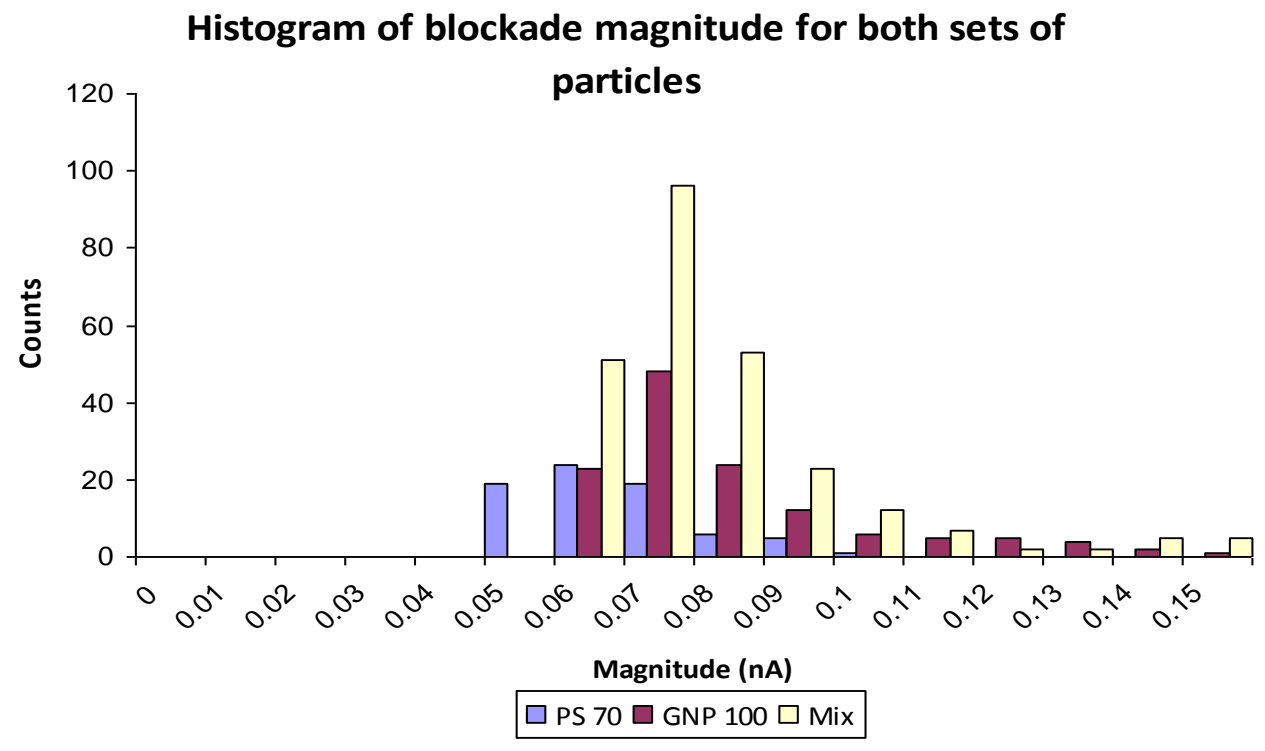

Figure 4.23: Distribution of blockade magnitudes of $70 \mathrm{~nm}$ polystyrene nanoparticles, $100 \mathrm{~nm}$ gold nanoparticles and a mixture of polystyrene and gold nanoparticles. The expected bimodal distribution is not observed in this measurement with the absence of the double peaks as observed in section 3.2.2.2. 
Figure 4.23 shows the distribution of the magnitudes of the blockade events detected by the instrument. The blue bar indicates distribution for the $70 \mathrm{~nm}$ polystyrene nanoparticles, the purple bar for $100 \mathrm{~nm}$ gold nanoparticles and the yellow bar signifies the distribution for the measurements taken for the mixture of gold and polystyrene nanoparticles.

As it has been shown in chapter 3 , the distribution of nanoparticles will converge to give a bell-shaped distribution. Figure 4.23 shows that the nanoparticles detected follow that trend; $70 \mathrm{~nm}$ peaks at a magnitude of $0.06 \mathrm{nA}, 100 \mathrm{~nm}$ peaks at $0.07 \mathrm{nA}$, and the mixture of both polystyrene and gold nanoparticles gives a peak at $0.07 \mathrm{nA}$. The peaks for both polystyrene and gold nanoparticles that were detected on separate occasion are as expected i.e. blockade magnitude from $100 \mathrm{~nm}$ gold nanoparticles is larger than that of $70 \mathrm{~nm}$. However, a bimodal distribution was expected for the measurement of the mix of nanoparticles but this is not the case. The peak for the measurement of the particle mix lies on $0.07 \mathrm{nA}$, which indicates that most of the nanoparticles detected are of $100 \mathrm{~nm}$ gold nanoparticles and not many of the $70 \mathrm{~nm}$ polystyrene nanoparticles. The loss of the $70 \mathrm{~nm}$ polystyrene peak is suspected to be from an unbalanced mix of the two sets of nanoparticles, and that there are more gold nanoparticles in the mix as compared to the polystyrene nanoparticles.

To determine if the instrument can identify the two different nanoparticles, the duration data for both nanoparticles are analysed. This is because the time taken for the particle to translocate through the nanopore depends on the surface charge of the nanoparticles [34]. Figure 4.24 shows the duration distribution of polystyrene and gold nanoparticles as well as the mixture of the two sets of nanoparticles. 


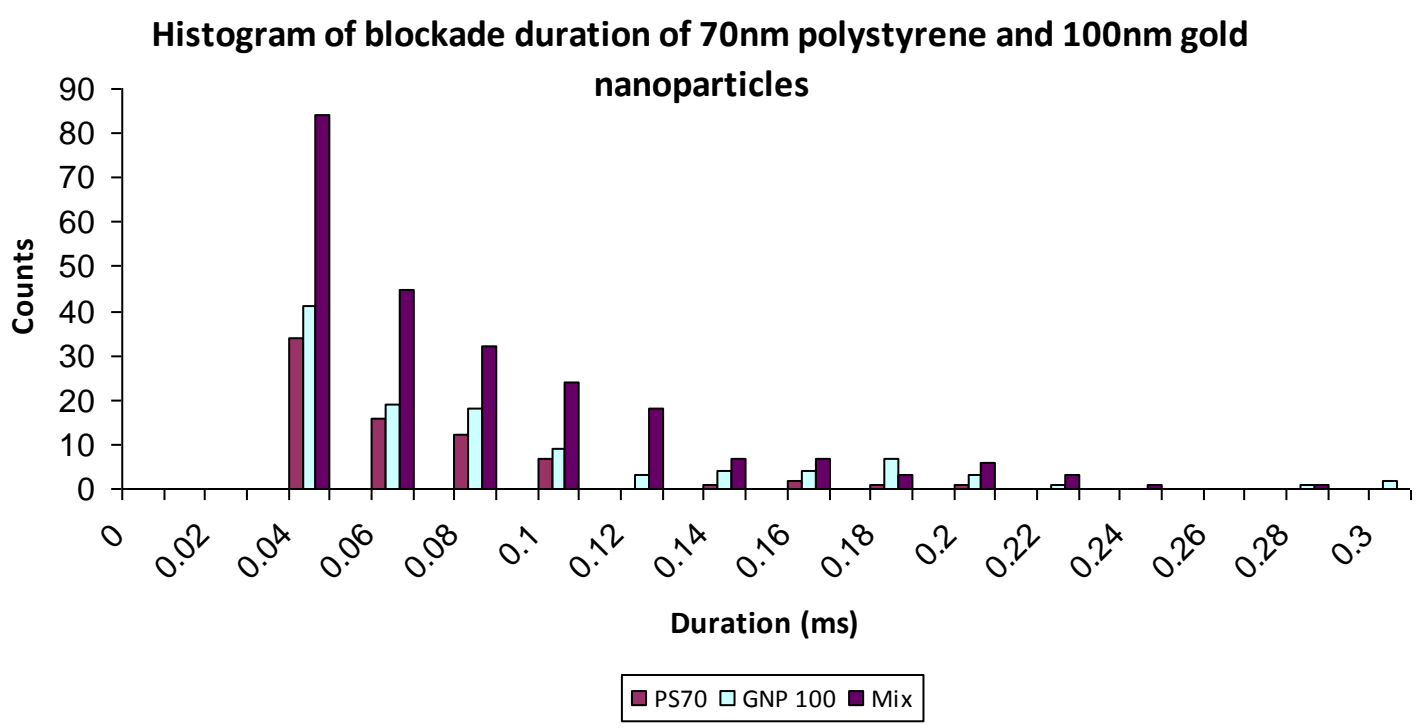

Figure 4.24: Distribution of the time taken for both the polystyrene and gold nanoparticles to go through the nanopore. The absence of two distinctive peaks for the mixture solution showed that particle identification is yet to be established.

From figure 4.24, the histograms show that all the nanoparticles' translocation time peaks at a value of $0.04 \mathrm{~ms}$. Here; it is assumed that gold nanoparticles have a higher surface charge than polystyrene particles as it is metallic. The particle mobility in the solution is influenced by the surface charge in electrophoretic measurements. As such, It is expected that the gold nanoparticles would travel through the nanopore at a faster rate but as seen in figure 4.24 , this is not observed. This result suggests that the instrument cannot distinguish between two different species of nanoparticles measured at the same time.

\subsubsection{Discussion}

It has been proven that the instrument is able to resolve two different diameters of nanoparticles, but from these measurements, the instrument is unable to resolve the types of nanoparticles. However, this observation is unique to the setting conditions for the measurements done. In this case, the result shows that the duration of particle translocation is not enough to show that the two nanoparticles analysed are of different species. Ito et al. [5] studies the effect of two polystyrene particles having different surface $-\mathrm{COOH}$ density. Both these particles have similar diameter however one particles set has a surface density of $24,220-\mathrm{COOH}$ groups per particle and the 
other has $120-\mathrm{COOH}$ groups per particles. The measurement was conducted using carbon nanotubes as the pore and results showed that there is appreciable difference in the translocation duration between the two sets of particles, which suggests that surface charge plays an important role in aiding mobility of particles through the pore. However, the study showed that the higher charged polystyrene particles moves through the pore at a slower time. Ito et al. [5] attribute this to a few factors which include a failure of their approximation at high surface density and a lowered effective surface charge due to the positive counter ions present in the solution that doesn't necessarily associate with the carboxyl group - which adds to the screening effect.

Success at discriminating between gold and polystyrene particles in the same solution is yet to be achieved. Further exploration would be useful in order to distinguish the two types of nanoparticles present in the same solution.

\subsection{Measurement of gold nanoparticles under Variable Pressure Module (VPM)}

Another method to detect gold particles in this study is the use of Variable Pressure Module (VPM). VPM is a new addition to the present technology where water based manometer is used to give pressure to the fluid cell on top of the electrophoresis already present in the technology. Utilizing this add-on to the system will assist in detecting metal nanoparticles using qNano as the metal nanoparticles will be under two operating forces; pressure applied and electrophoresis.

Blockade events detected in section 4.3 are assumed to be gold aggregates instead of single particle events [89]. Section 4.3 shows that under pure electrophoresis, gold nanoparticles moves at a slower rate through the nanopore. To substantiate this observation, section 4.3.1 shows that the numbers of detection events are exceptionally low as compared to polystyrene nanoparticles as shown in Chapter 3 (about $10 \%$ of the counts for polystyrene) within the same recording duration. 


\subsubsection{Experimental Methods}

In this experiment, $100 \mathrm{~nm}$ gold nanoparticles purchased from BB International were used, as they are the largest gold nanoparticles available to use in this research. These nanoparticles were suspended in IZON's buffer solution. Figure 4.25 is the image of gold nanoparticle purchased from BB International.

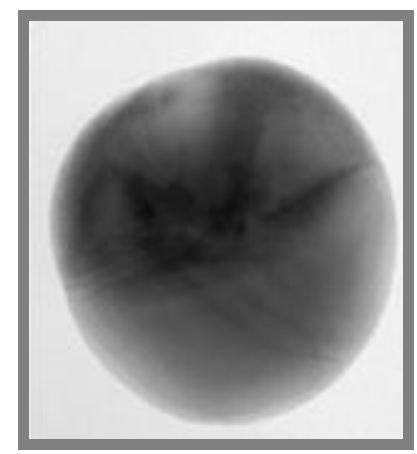

Figure 4.25: Image of $100 \mathrm{~nm}$ particles purchased from $B B$ International. Photo from BB International.

IZON's $100 \mathrm{~nm}$ polystyrene nanoparticles suspended in the standard buffer solution were used as a reference to find the suitable stretch range for detection of $100 \mathrm{~nm}$ gold nanoparticles. The polystyrene nanoparticles were dispersed in a 1:400 dilution. Gold nanoparticles used were suspended in the buffer solution in increasing concentrations of 1:100, 1:50, and 1:10. The increasing concentration of gold nanoparticles was used to investigate the relation between blockade counts and concentration of nanoparticles in the buffer solution. Supporting material retrieved from BB International shows that $100 \mathrm{~nm}$ particles has a particle concentration of $5.6 \times 10^{9}$ particles $/ \mathrm{ml}$.

Mixtures of gold nanoparticles and buffer were sonicated for approximately 20 minutes prior to use. The aperture and conditions were systematically tested to determine the optimum running condition for this experiment. A detailed summary of the running conditions are given in Table 4.4. 


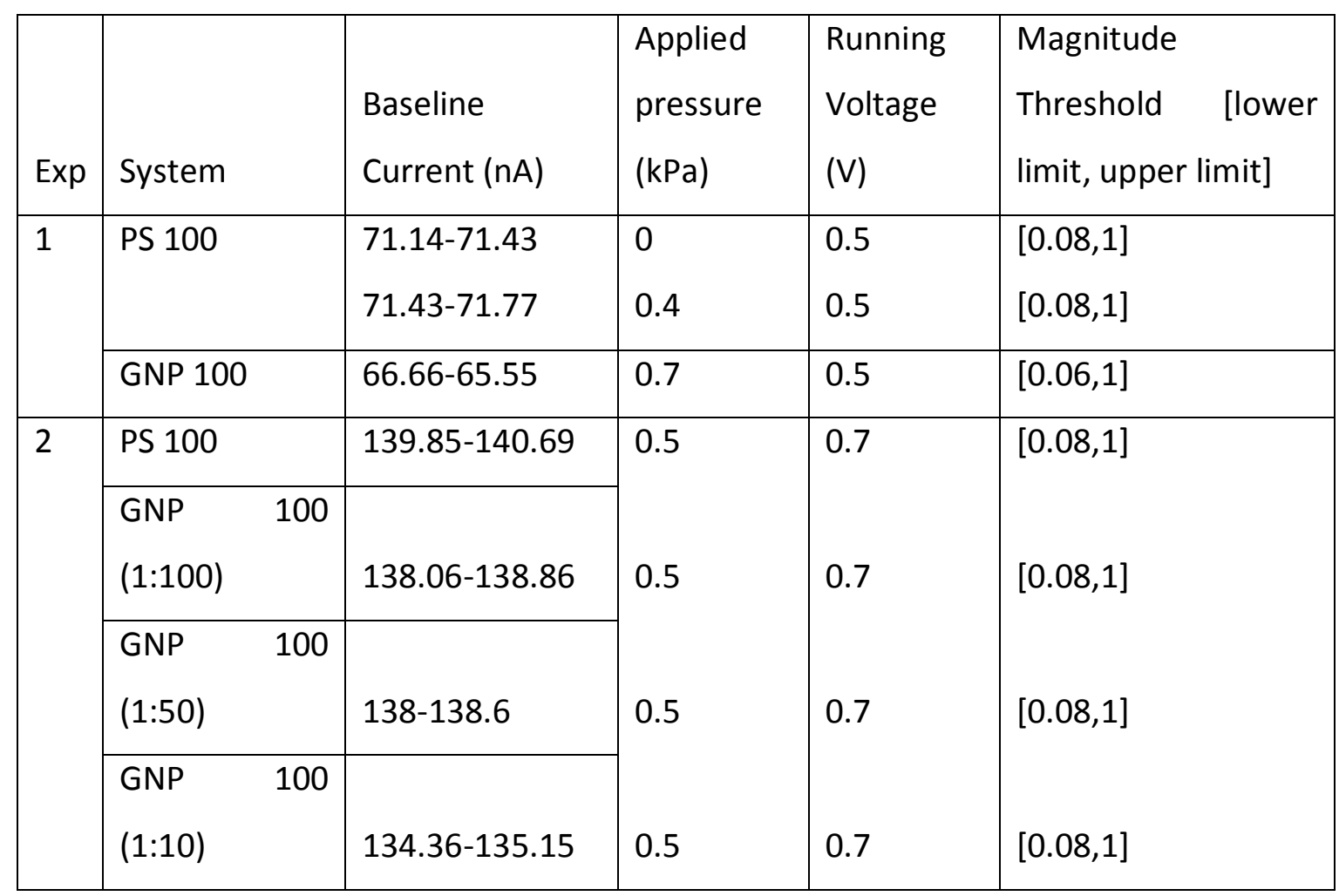

Table 4.4: Running conditions for polystyrene and gold nanoparticles using VPM.

The abbreviation PS refers to polystyrene particles and GNP refers to gold nanoparticles. Values for applied pressure and running voltage show the amount needed for the nanoparticles to run at a satisfactory rate. Magnitude threshold is the set lower and upper limit that determines blockade events that will be recorded. This value depends on the level of background noise.

Experiment 2 in table 4.4 shows the optimum conditions for ideal measurements. The conditions, which was set at $0.7 \mathrm{~V}$ with the threshold setting set at $[0.08,1] \mathrm{nA}$. A pressure of $0.5 \mathrm{kPa}$ was used for both polystyrene and gold nanoparticles. The detection event was expected to be a resistive pulse for both polystyrene and gold nanoparticles as the surface charge for both is a negative value.

\subsubsection{Results and Analysis}

IZON's $100 \mathrm{~nm}$ polystyrene particles were used to calibrate the system and determine the suitable stretch for measurements of gold nanoparticles. Figure 4.26 show a screenshot of recorded blockade events for $100 \mathrm{~nm}$ polystyrene particles. 


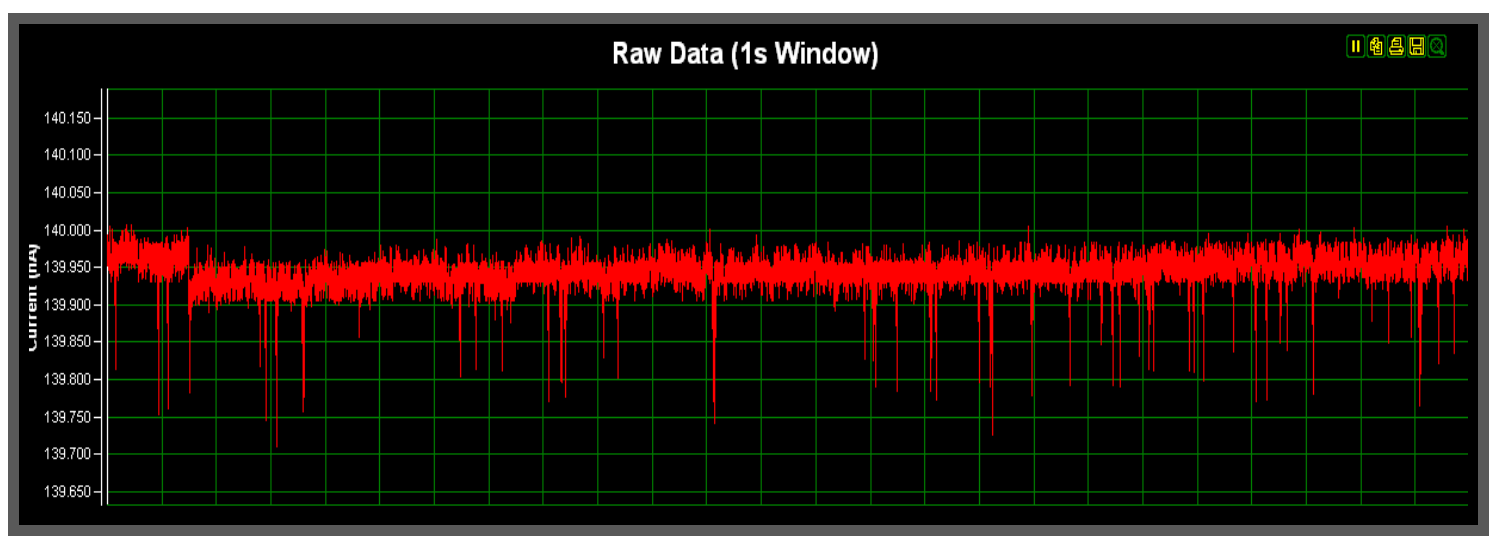

Figure 4.26: Screenshot of $100 \mathrm{~nm}$ polystyrene nanoparticles going through the nanopore.

Figure 4.27 shows the distribution of the polystyrene particle. The recorded blockade rate was approximately 3000 counts/min.

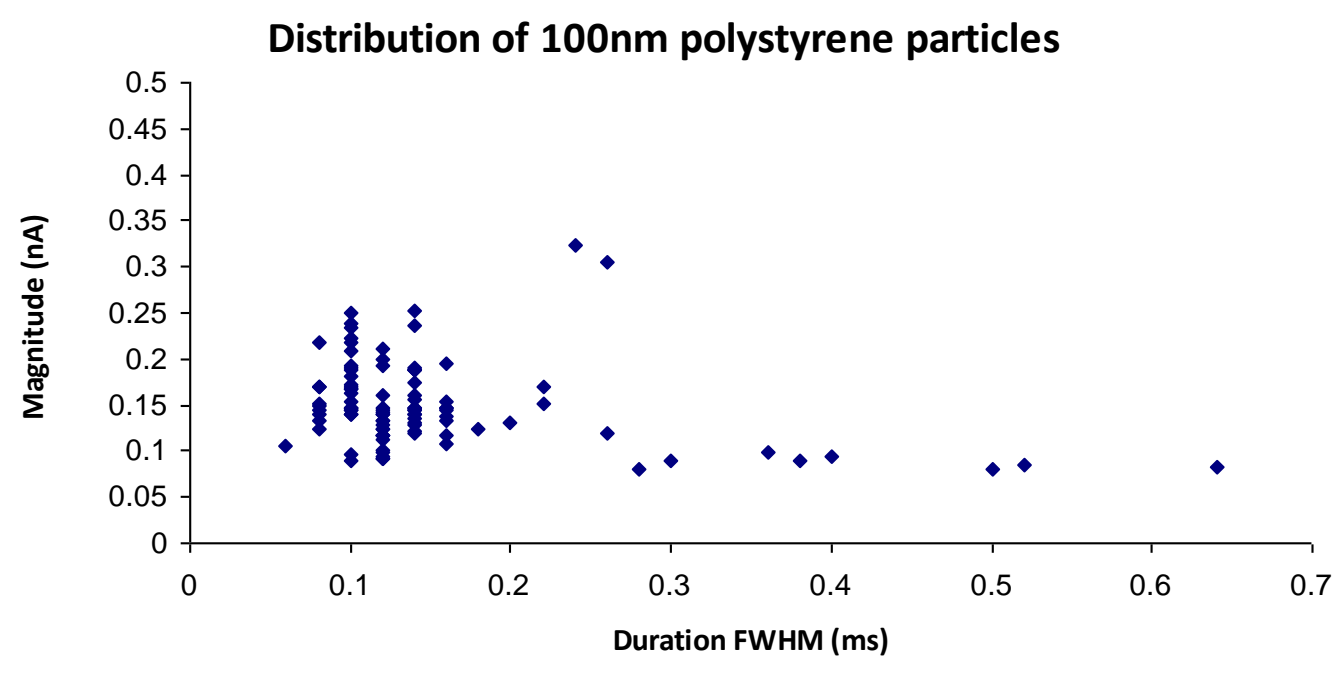

Figure 4.27 Distribution of selected $100 \mathrm{~nm}$ polystyrene nanoparticles suspended in IZON's buffer.

Figure 4.27 shows the selected blockade events plotted by blockade magnitude as a function of duration at half of the depth of the blockade magnitude (Duration FWHM). As explained in section 2.1, the 'Duration' data calculates the time taken for the normalised trace signal to cross a particle blockade depth downwards and upwards again. As such, calculation for 'Duration' depends heavily on the blockade analysis threshold settings particularly the lower threshold limit. This means that if the magnitude threshold was changed, the software will record a different translocation 
duration. For this reason, the 'Duration' time was not used to plot the particle distribution. However, 'Duration FWHM' is the time taken at half of the particle blockade depth. The software plots duration FWHM as a standard duration as it is independent of the blockade analysis threshold settings. Therefore, this means that comparison between data points can be done compared consistently.

There is an increase of blockade rate with the use of the pressure system as it adds to the driving force that moves the nanoparticles through the nanopore. Figure 4.27 shows the selected distribution of blockade events. Data points concentrated at the lower left hand corner of the graph generally indicate that the sample used is monodispersed.

The fluid cell was cleaned and flushed with the buffer solution to ensure that the system is free from polystyrene nanoparticles. With the aperture stretch and settings determined, $100 \mathrm{~nm}$ gold nanoparticles were introduced to the system in an increasing concentration of $1: 100,1: 50$ and $1: 10$. This was done to avoid blockage of the nanopore and residual contamination from the more concentrated sample. Results are plotted in a graph of blockade magnitude against duration as in figure 4.28.

Blockade events of $100 \mathrm{~nm}$ polystyrene and gold nanoparticles at different dilution

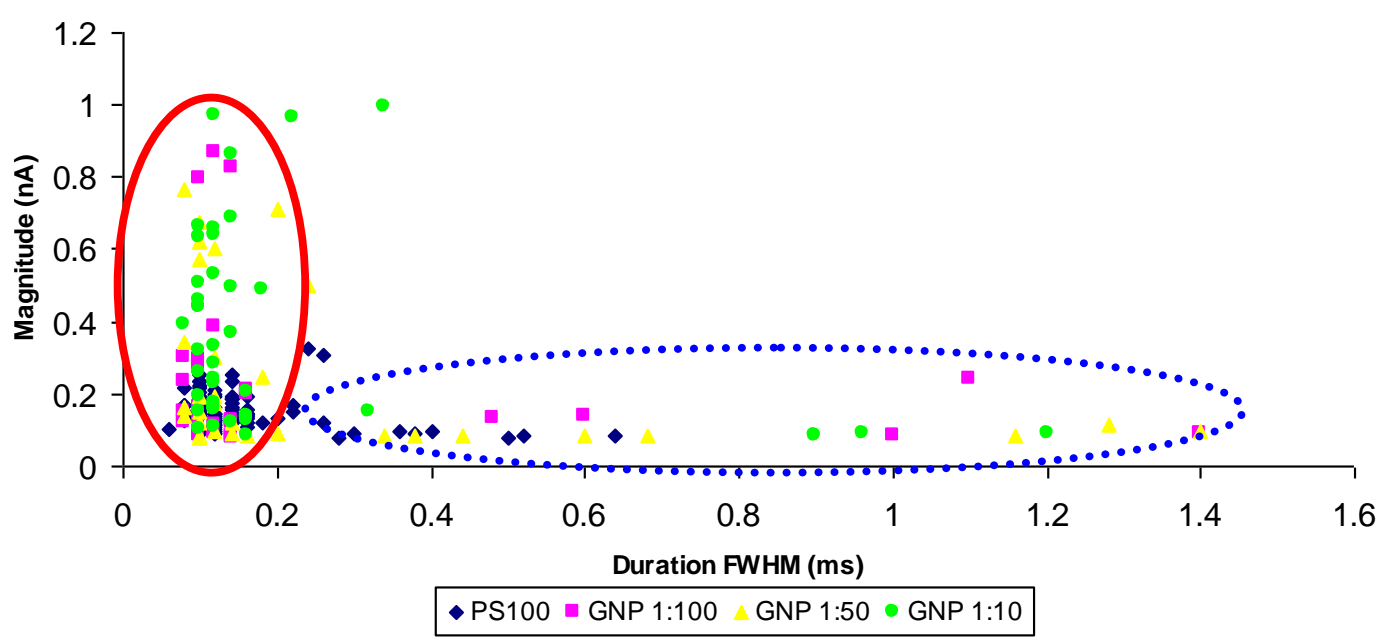

Figure 4.28: Gold nanoparticles at varying concentration have a larger blockade magnitude than the polystyrene particles as denoted in the red bubble, which suggest the presence of aggregated gold particles. The plot also shows that $90 \%$ of the translocated particles have a narrow distribution of translocation duration. 
Figure 4.28 show that the majority of both polystyrene and gold nanoparticles take approximately 0-0.2 ms to go through the nanopore (as shown in the solid red bubble). This observation suggests that surface charge has minimal effect on the speed of translocation with the use of the pressure system. However, some gold nanoparticles take a longer time going through the nanopore. This is seen in blockade events that go through the pore with a time longer than $0.3 \mathrm{~ms}$ (as shown in the dotted blue bubble).

Comparing the magnitude of both the polystyrene and gold nanoparticles in figure 4.28 , it can be seen that the polystyrene nanoparticles are concentrated in the region below $0.4 \mathrm{nA}$ with $80 \%$ of the polystyrene points lays in this region. On the other hand, the gold nanoparticles spread over a wider range of blockade magnitude i.e. 0.05 nA-1 $\mathrm{nA}$ with the majority, at less than $0.5 \mathrm{nA}$. This suggests that the polystyrene nanoparticles are more monodispersed in comparison to the gold nanoparticles.

It is especially important to be able to distinguish between these two species of nanoparticles. Success at achieving this will definitely be of advantage as it opens up a market for the qNano to be an excellent research instrument. Therefore, polystyrene and gold nanoparticles' translocation duration will be analysed to see if there is a difference in signal duration as an effect of its different surface charge. For this, the data from $\mathrm{X}$-axis in figure 4.28 is translated to give a distribution histogram of the duration of nanoparticles' translocation that is given in figure 4.29. 


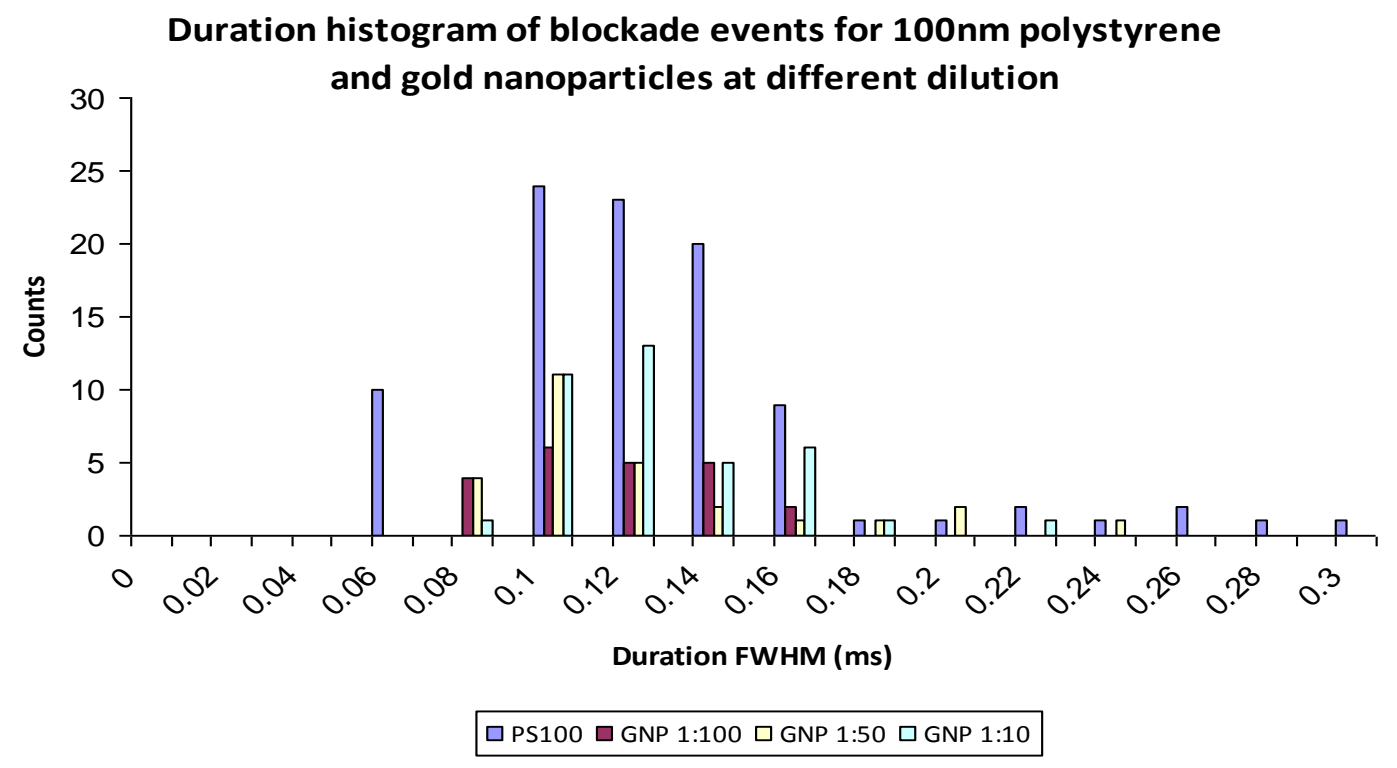

Figure 4.29: Histogram of blockade duration for polystyrene nanoparticles and gold nanoparticles at different concentration.

Figure 4.29 shows a distribution of the time it takes for the nanoparticles to go through the nanopore. The average time taken for both sets of nanoparticles with dilutions of 1:100 and 1:50 are approximately $0.1 \mathrm{~ms}$. However, the average time taken for a dilution 1:10 are approximately 0.13 ms which suggests that the gold nanoparticles at this concentration have a lower particle mobility. This might be due to the varying surface charge of aggregated particles as compared to single particles. Other factors such as screening as the size of particles also play a role in establishing the particles' mobility.

Measurements for the gold nanoparticles suspended inthe buffer were made in an increasing dilutions of $1: 100,1: 50$, and $1: 10$. In theory, the number of single particle events that should get detected between a dilution of $1: 100$ to $1: 10$ is expected to increase ten-fold but this does not apear to be so as shown in figure 4.30. 


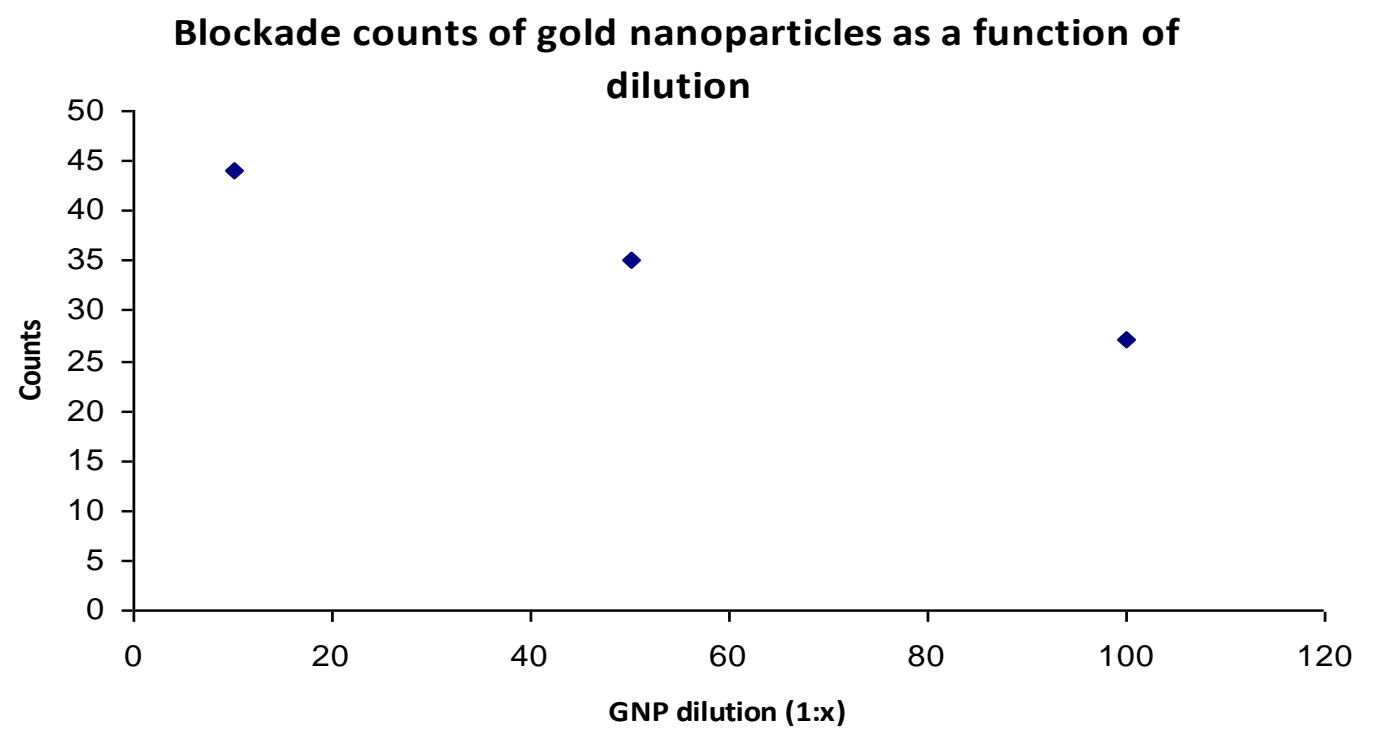

Figure 4.30: Blockade counts as particle dilution gets less concentrated. X-axis denotes the gold nanoparticles' dilution. The figure shows that the blockade count increases as the concentration of the gold nanoparticles dilution increases.

Graph in figure 4.30 indicate that the number of translocated nanoparticles for a dilution of $1: 10$ is less than ten-fold compared to nanoparticles with a 1:100 dilution. This could imply that the blockades detected are not single particle events. Thus, it can be concluded that aggregation of the gold nanoparticles may have occurred in the solution. This aggregation is induced by the presence of potassium chloride $(\mathrm{KCl})$ salt in the standard electrolyte buffer solution $[49,52,55,57,58,59,64]$. This formation of gold aggregates in the solution could explain why high frequency of gold nanoparticles translocation was not observed compared to those of polystyrenes nanoparticles. This can be demonstrated in figure 4.26, where blockade events of single polystyrene nanoparticles were recorded. However, the same cannot be said for the gold nanoparticles, as demonstrated in figure 4.31. 

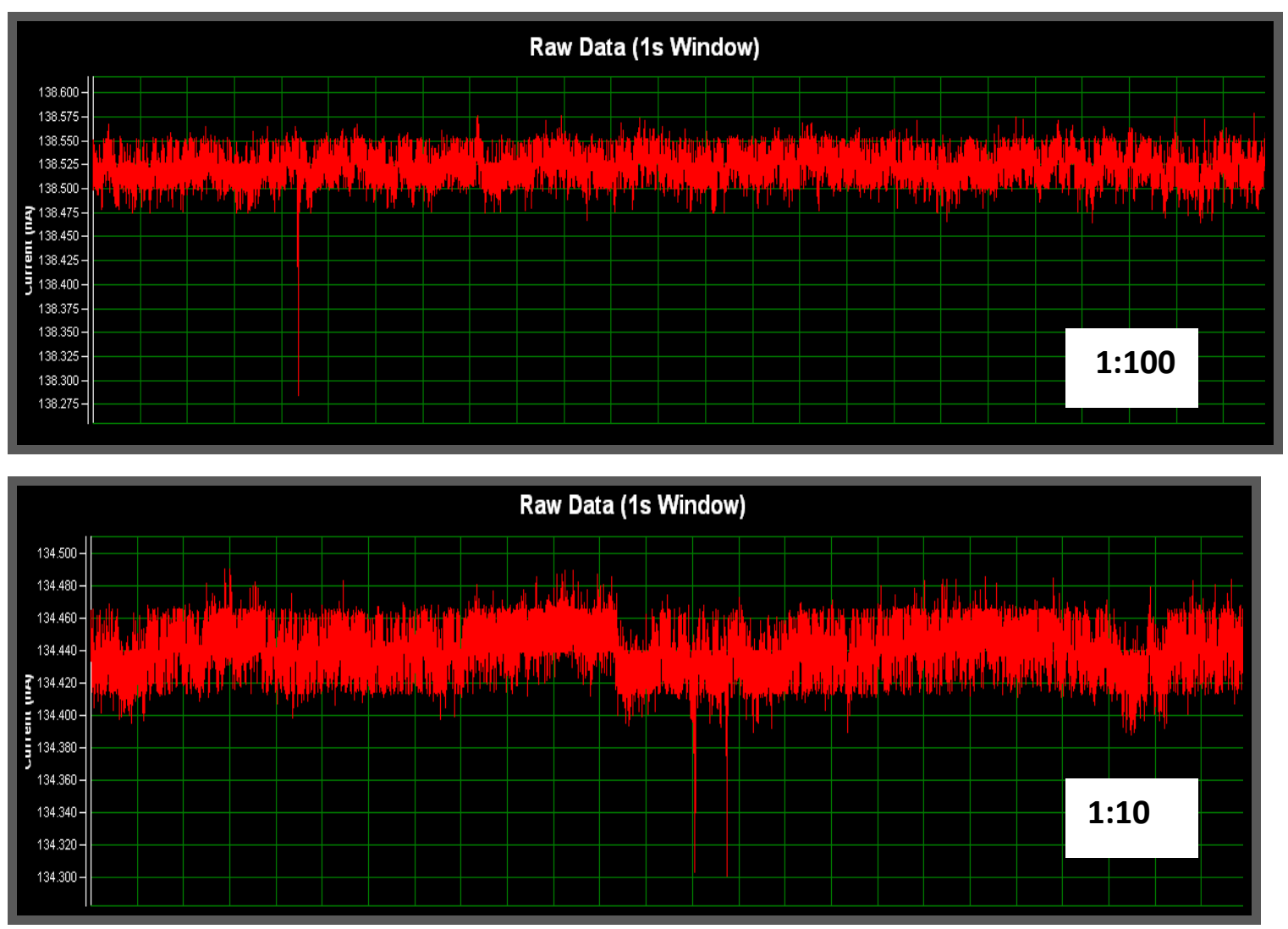

Figure 4.31: Blockade events of gold nanoparticles in a 1:100 and 1:10 dilution suspended in IZON's buffer solution. Screenshot shows that the blockade rate translocation for gold nanoparticles are much lower to that of polystyrene nanoparticles.

From this measurement, the blockades rate for the gold nanoparticle were approximately 10 blockades/minute, which is much less compared to the rate of polystyrene particles which can reach up to 4000 blockades/minute. This slow travelling of gold nanoparticles suspended in IZON's buffer solution was assumed due to the change in volume of the gold aggregates, which might affect the overall surface charge of the aggregates in the buffer solution. The effect of aggregation of gold nanoparticles in the buffer system can be observed in figure 4.32 . 


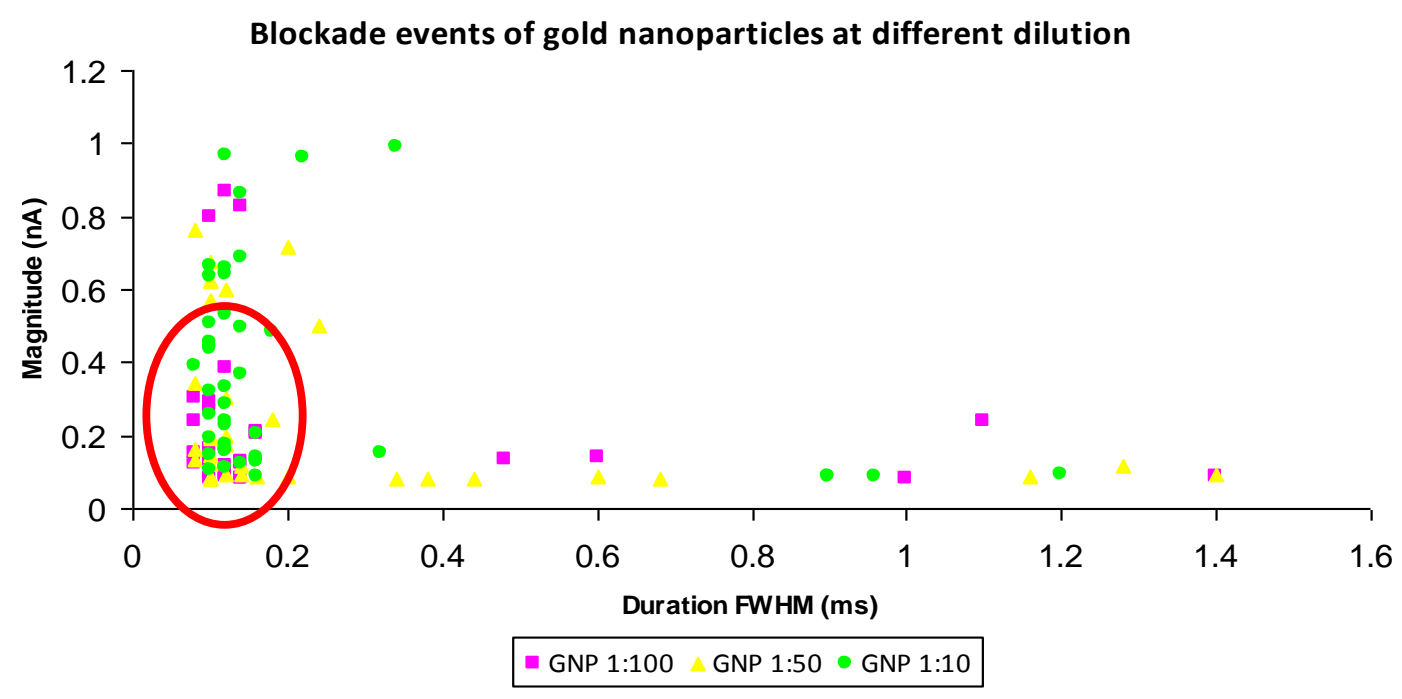

Figure 4.32: Blockade events of $100 \mathrm{~nm}$ gold nanoparticles at different dilution. There seem to be increasing counts of larger aggregates as concentration of gold nanoparticles is increased.

Figure 4.32 shows the distribution of gold nanoparticles with data in pink representing gold nanoparticles dilution at 1:100, yellow with 1:50, and green with 1:10 dilution. Comparing figure 4.28 and figure 4.32 , the majority of the gold nanoparticles' data points in the solid red bubble of figure 4.32 have a magnitude less than $0.4 \mathrm{nA}$ and passes through the pore at a time less than $0.2 \mathrm{~ms}$, which is where most data points for polystyrene nanoparticles are positioned. This could indicate that most of the gold nanoparticle blockades detected was single particle events as they overlap with the polystyrene blockades as shown in figure 4.28. The blockades that are beyond the described region could mean that the blockades detected are aggregates of gold and not single particle events.

Figure 4.32 also shows that the number of larger magnitude blockades increase as the concentration of gold increases. This suggests that the concentration of gold nanoparticles diluted in the standard buffer determines the quantity of aggregates formed. The formation of aggregates in turn depends on how concentrated the gold nanoparticles are relative to the concentration of $\mathrm{KCl}$ present in the standard buffer solution. 


\subsubsection{Discussion}

In section 4.3, nanoparticles move under the influence of electrophoresis, the main driving force that pushes the nanoparticles through the nanopore. This mechanism mainly relies on the value of the nanoparticles' surface charge. Hence, the time it takes to travel through the pore depends on the strength of the nanoparticles surface charge. Thus, the difference between two species of nanoparticles can be analysed by investigating the time to travel through the pore. However, with the addition of pressure on top of electrophoresis as the nanoparticles' driving force, there is little difference in the duration time for the particle to travel through the pore. Such an effect suggests that the influence of pressure overcomes the influence of electrophoresis and has been shown experimentally in figures 4.28 and 4.29 [34]. Further analysis of the blockade data shows that minimal difference of translocation duration was observed for both types of nanoparticles. This is shown in figure 4.29 where the mode for particle duration for polystyrene and gold nanoparticles (regardless of concentration) is recorded at $0.1 \mathrm{~ms}$. Gold nanoparticles suspended in a 1:10 dilution however shows a different trend.

It has been demonstrated that aggregates of gold that are capped with citrate forms when suspended in IZON's standard buffer solution. This observation is also shown in section 4.3 when gold nanoparticles are run under pure electrophoresis. This is a consequence of the presence of $\mathrm{KCl}$ in the buffer solution that causes the gold to aggregates $[49,52,55,57,58,59,64]$. At present, it is not known what the optimum concentration of $\mathrm{KCl}$ in the standard buffer solution should be in order to prevent gold aggregates from forming. Philips [48] reported that in general, gold nanoparticles stabilized with citrate have a critical concentration before aggregation happens due to the presence of salt in the buffer solution. This aggregation is due to screening effect from chloride ions $\left(\mathrm{Cl}^{-}\right)$which hinders the electrostatic stabilization of the gold nanoparticles $[58,63]$.

Figure 4.30 is a plot of the blockade counts of gold nanoparticles as a function of concentration. The plot shows that increasing the concentration results in an increasing blockade counts. As stated in the experimental section, the $100 \mathrm{~nm}$ gold 
nanoparticles have a particle concentration of $5.6 \times 10^{9}$ particles $/ \mathrm{ml}$. Comparing to the counts of polystyrene nanoparticles, the counts for gold are much lower but the signals are very prominent as shown in figure 4.31 .

One possible reason could be that the pore was too small for the aggregated gold nanoparticles to pass through. Minimal studies have has been done to investigate the rate of aggregation of gold nanoparticles in IZON's standard buffer solution. In this study, the measurement of gold nanoparticles was done as soon as they were added to the buffer to avoid any gold aggregates and detect only single nanoparticles. However, no evidence can be provided to support this assumption yet. At this stage, it can only be suggested that the gold nanoparticles aggregate at a slow rate when suspended in buffer solution.

The concentration of the gold nanoparticles mixed in the standard buffer might also play a role in determining the size of the gold aggregates. Figure 4.32 shows that more blockades with higher magnitudes were present as the concentration increases. This is shown by blockade events that deviate upwards from a magnitude of $0.4 \mathrm{nA}$. This could mean that higher concentration of particles produce larger gold aggregates. Figure 4.33(a)-(c) shows the blockade events for each gold nanoparticle compared to $100 \mathrm{~nm}$ polystyrene particles. 
Blockade events of 100nm polystyrene and gold nanoparticles at 1:100 dilution

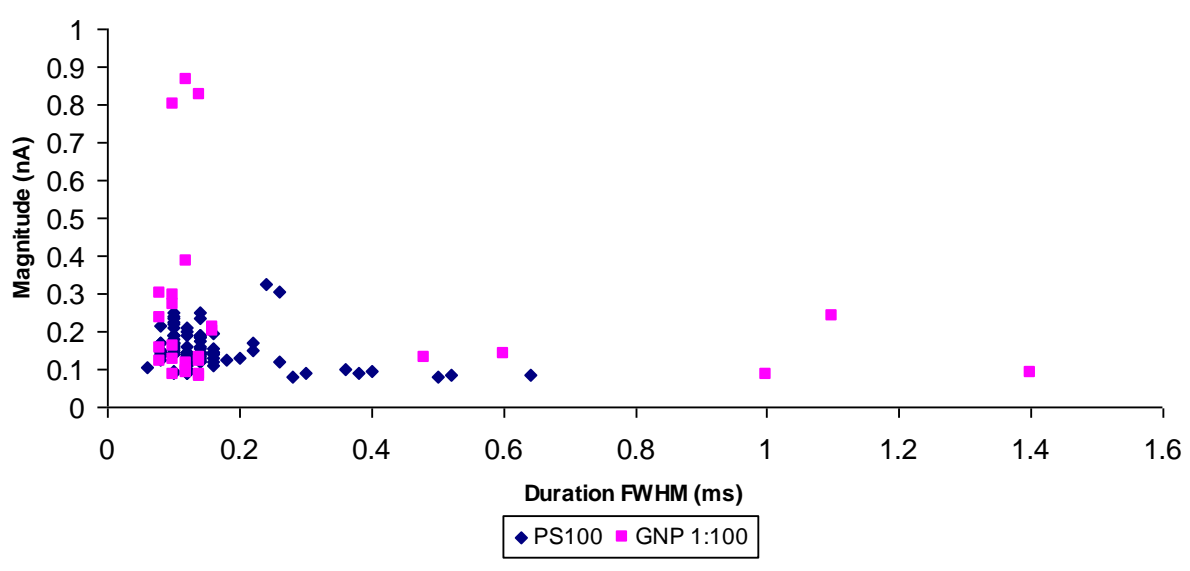

(a)

Blockade events of 100nm polystyrene and gold nanoparticles at 1:50 dilution

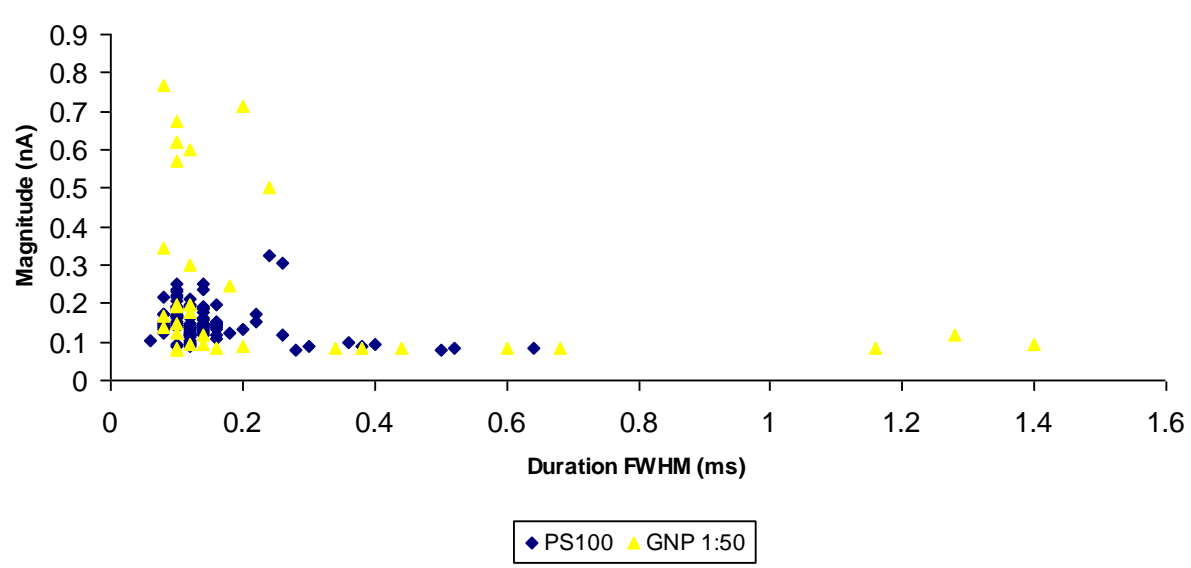

(b)

Blockade events of $100 \mathrm{~nm}$ polystyrene and gold nanoparticles at 1:10 dilution

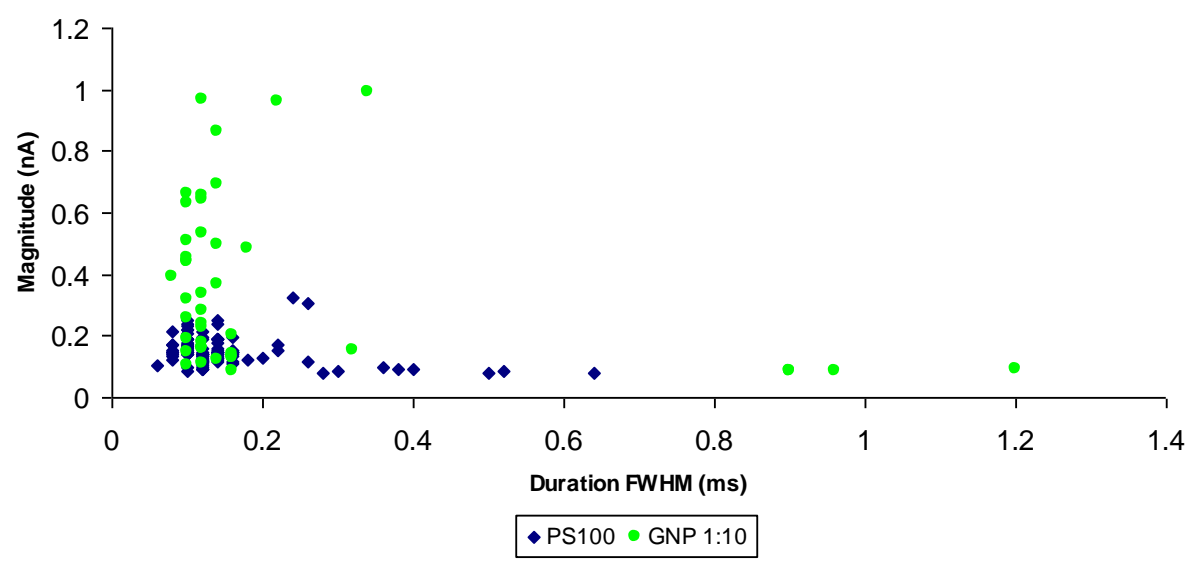

(c)

Figure 4.33(a)-(c): Plots of blockade events for gold in a dilution of 1:100, 1:50, and 1:10 with respect to $100 \mathrm{~nm}$ polystyrene particles. These plots shows larger aggregates are more likely to form with increasing gold concentration. 
As shown in figure 4.33, the count of gold nanoparticles blockades higher than $0.3 \mathrm{nA}$ increases as the concentration of gold nanoparticles increases. This suggests more aggregates formed with higher concentration of gold nanoparticles available in the buffer solution. In future work, the mechanism of gold aggregation in IZON's buffer system can be explored.

\subsection{Summary}

This chapter looked into the measurement of gold nanoparticles using the qNano technology. It was found that gold nanoparticles form aggregates when it is suspended in IZON's standard buffer solution (section 4.3, and section 4.4). These aggregates are suspected to form due to the presence of $\mathrm{KCl}$ salt in IZON's standard buffer solution and this assumption was investigated (section 4.3.2). It was found that the concentration of both gold nanoparticles and $\mathrm{KCl}$ in the buffer system plays a role in determining the rate of formation of aggregates as shown in section 4.3.3. An upgrade of the bandwidth of the qNano instrument (from $10 \mathrm{kHz}$ to $50 \mathrm{kHz}$ ) opens up the possibility to detect nanoparticles with a mean diameter of between $50 \mathrm{~nm}$ and 100 $\mathrm{nm}$ with no loss of data from the measurements (section 4.3.4). With this higher bandwidth available to the instrument, particle blockades can be detected more accurately. Subsequently, the frequency of particle blockades when the concentration of gold nanoparticles was changed was also looked into (section 4.3.5). Section 4.3.6 looked at qNano's ability to differentiate between two species of particles, which are the polystyrene and gold nanoparticles using the particle's duration of translocation due to the surface charge of the particles in the solution. This chapter has shown that gold nanoparticles can be detected and the next chapter looks into the possibility of detecting other metal nanoparticles. 


\subsection{MEASUREMENTS OF OTHER INORGANIC AND NOBLE METALS NANOPARTICLES}

\subsection{Introduction}

In this chapter, brief investigation into the measurements of other types of metals particularly noble metals like silver nanoparticles and magnetic nanoparticles using qNano will be discussed. The silver nanoparticles used were from Eric Le Ru's research group [79], while the magnetic nanoparticles used were iron (II, III) oxide that has been coated with the water-soluble dextran. Additionally, silica nanoparticles were synthesized using method developed by Lindberg et al. [70] and Deng et al [93]. The aim of this chapter is to see the types of signals that are produced when both metals are used and whether this technology can be used further as another tool to characterise these nanoparticles.

\subsection{Silica particles}

The silica particles used were synthesized to give a range of diameters so that it can be referenced with the polystyrene particles supplied by IZON, which is between $100 \mathrm{~nm}$ to $780 \mathrm{~nm}$ as stated in Chapter 3.

\subsubsection{Synthesis of silica spheres}

The silica particles used in this research was synthesized using the well-known Stöber method to induce growth of the silica particles [66-68, 70, 72-75]. The two methods further developed by Lindberg et al. and Deng et al. [70, 93]. Three experiments were conducted using the Lindberg et al. [70] method while two experiments were done with Deng et al. [93] method. Tetraethyl orthosilicate (TEOS) undergoes a basecatalysed hydrolysis using ammonia solution $[69,70,71]$. The rate of reaction and extent of hydrolysis depends mainly on the concentration of the base/acid catalyst, in this case the ammonia solution [73]. Bogush and Zukoski [73] reports that in acidic conditions, the hydrolysis occurs at a faster rate compared to reactions in basic 
conditions. This suggests that in basic conditions, the rate of condensation is faster than the rate of hydrolysis.

\subsubsection{Method}

Synthesis of silica particles using the method developed by Lindberg et al. [70] which is summarised in table 5.1 as experiment 1, 2, and 3 . This method uses $35 \%$ ethanol (EtOH) which was added to ammonia $\left(\mathrm{NH}_{3}\right)$ and a salt solution of $0.1 \mathrm{mM}$ of sodium chloride $(\mathrm{NaCl})$ and deionised water. The solution was stirred for 30 minutes before tetraethyl orthosilicate (TEOS) was added to the stirring solution. The solution was then left to react and conditions are given in table 5.1.

The other method to produce silica particles were given by Deng et al. [93] which is summarised in table 5.1 as experiment 4 and 5 . This method requires two sets of solutions (here termed solution A and solution B) to be prepared. Solution A consist of TEOS mixed with EtOH. Solution B consists of deionised water that is mixed with $\mathrm{NH}_{3}$ solution in an EtOH solution. Solution B was stirred for 10 minutes before Solution A was added to the stirring solution. Reaction was left to react for 6 hours at the designated temperature as given in table 5.1.

All reagents were used without further purification and the product were purified via centrifuging it twice at 5000rpm for 15 minutes and washed with EtOH.

\begin{tabular}{|l|l|l|l|l|l|l|}
\hline Exp & $\begin{array}{l}\text { EtOH } \\
(\mathrm{ml})\end{array}$ & $\begin{array}{l}\mathrm{NH}_{3} \\
(\mathrm{M})\end{array}$ & $\begin{array}{l}\mathrm{H}_{2} \mathrm{O} \\
(\mathrm{M})\end{array}$ & $\begin{array}{l}\text { TEOS } \\
(\mathrm{M})\end{array}$ & $\begin{array}{l}\text { Time } \\
\text { (hours) }\end{array}$ & $\begin{array}{l}\text { Temp } \\
\left({ }^{0} \mathrm{C}\right)\end{array}$ \\
\hline 1 & 60 & 0.2 & 20 & 0.1 & 24 & 20 \\
\hline 2 & 60 & 1 & 13 & 1 & 24 & 20 \\
\hline 3 & 100 & 0.2 & 20 & 1 & 4 & 40 \\
\hline 4 & 36.8 & 1 & 2.3 & 0.3 & 6 & 20 \\
\hline 5 & 5 & 13 & 15.9 & 4.5 & & 60 \\
\hline
\end{tabular}

Table 5.1: Reaction conditions for the synthesis of silica particles. 


\subsubsection{Risk Assessment}

Table 5.2 list the risk assessment of the chemicals used to make the silica particles [91].

\begin{tabular}{|c|c|c|}
\hline Chemicals & Risk & Danger \\
\hline \multirow[t]{5}{*}{ TEOS } & \multirow[t]{5}{*}{ High } & Harmful if inhaled. \\
\hline & & Skin and eye irritant. \\
\hline & & $\begin{array}{l}\text { Long-term exposure may cause kidney or } \\
\text { liver damage. }\end{array}$ \\
\hline & & $\begin{array}{l}\text { Typical Threshold Limit value (TLV) - Time } \\
\text { Weighted Average (TWA): } 10 \text { ppm. }\end{array}$ \\
\hline & & $\begin{array}{l}\text { Typical Short Term Exposure Limit (STEL): } 30 \\
\text { ppm. }\end{array}$ \\
\hline \multirow[t]{3}{*}{$\mathrm{NH}_{3}(35 \%)$} & \multirow[t]{3}{*}{ Poison } & Corrosive \\
\hline & & May be fatal if swallowed or inhaled. \\
\hline & & $\begin{array}{l}\text { Mist and vapour cause burns to every area of } \\
\text { contact. }\end{array}$ \\
\hline $\mathrm{NaCl}$ & Low & Causes eye irritation. \\
\hline
\end{tabular}

Table 5.2: Risk evaluation

\subsubsection{Results and Analysis}

The samples were characterised using JEOL 6500F Scanning Electron Microscope (SEM) as well as SemAfore software for particle size distribution analysis. All images are taken at the same magnification of $\times 20,000$.

\section{Experiment 1 and experiment 2}

Figure 5.1 shows the images of the synthesized silica particles for experiments 1 and 2 . 


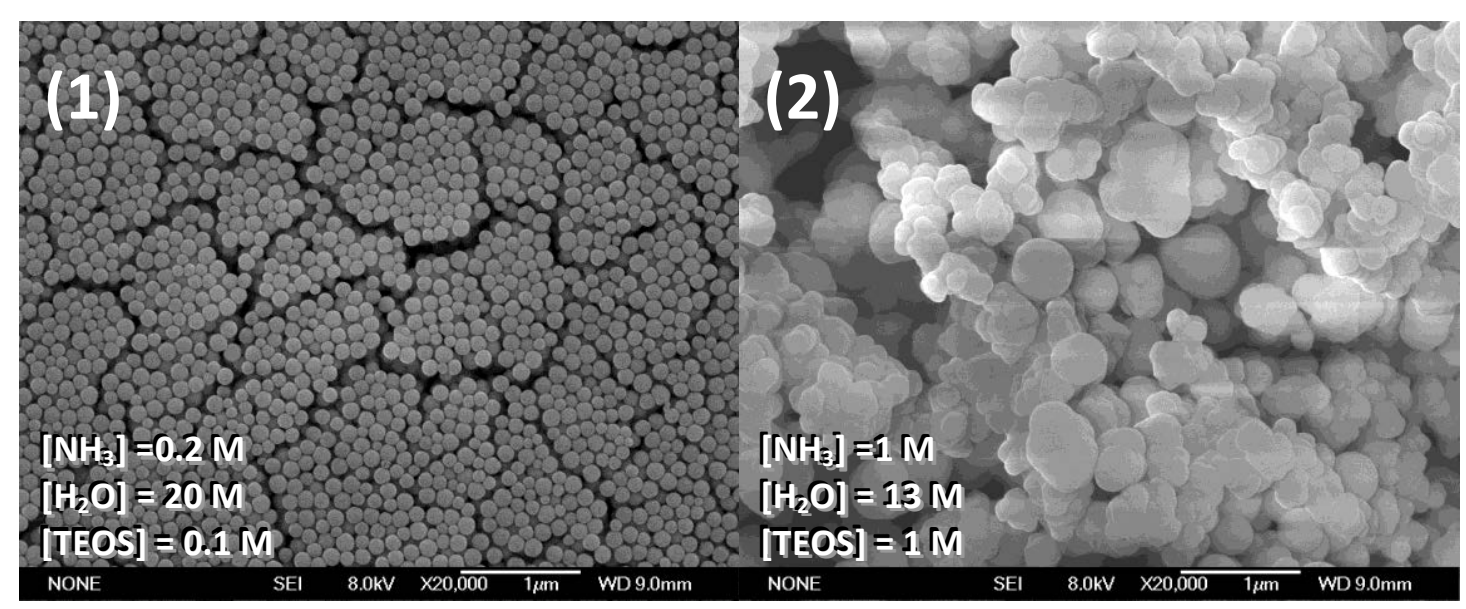

Figure 5.1: SEM images of silica particles at $\times 20,000$ magnification. Sample from Experiment 2 shows the presence of silica particles aggregates.

Comparing experiment 1 and 2 in figure 5.1, it can be seen that changing the concentration of $\mathrm{NH}_{3}, \mathrm{H}_{2} \mathrm{O}$ and TEOS used gives a huge change in the product. Spherical particles are produced in experiment 1 with a monodisperse distribution. Conversely, a higher concentration of both ammonia and TEOS used in experiment 2 produce non-uniform and aggregated particles. In addition, the average diameter of the particles in experiment 2 seems to be much bigger than the samples from experiment 1 . Observation for experiment 2 saw an immediate change of colour of the solution upon addition of TEOS, i.e. from colourless to a milky white solution. At the end of experiment 2, silica particles formed precipitate out from the solution. A clear solution formed on the top and white solid at the bottom of the reaction vessel.

\section{Experiment 1 and Experiment 3}

Figure 5.2 below shows the result from experiment 1 and 3. Experiment 3 was done using the same method used to synthesize particles in experiments 1 and 2 . 


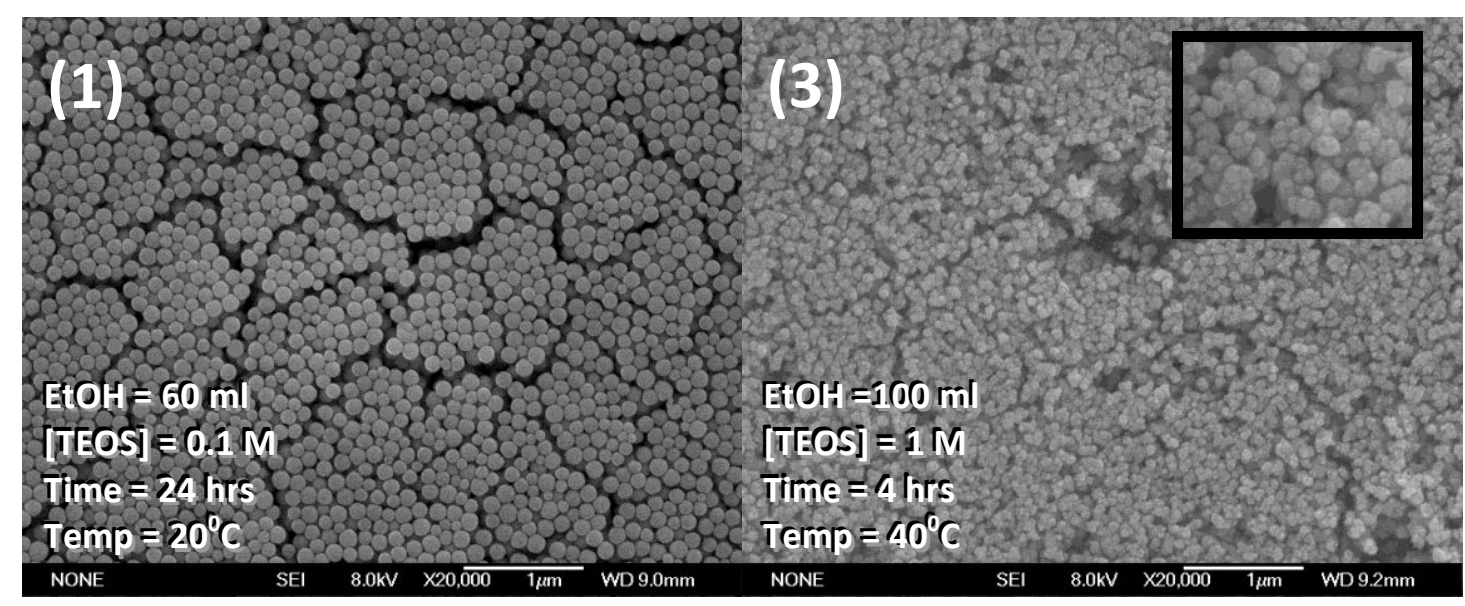

Figure 5.2: SEM images of silica particles at $\times 20,000$ magnification. Reaction conditions for Experiment 3 shows that it produces finer silica aggregates.

Comparing experiment 1 and experiment 3, the difference in the amount of EtOH, TEOS, reaction duration, and temperature of reaction used shows differences in the product. An image from sample 3 shows particles have aggregated (as shown in insert) with higher TEOS concentration and reaction temperature used. The reaction time however was much shorter than samples from experiment 1 and could play a role in the quality of the particles produced.

Because samples from experiment 1 show to be the most uniform in shape and size, a size distribution histogram can be constructed using SemAfore. This is given in figure 5.3 .

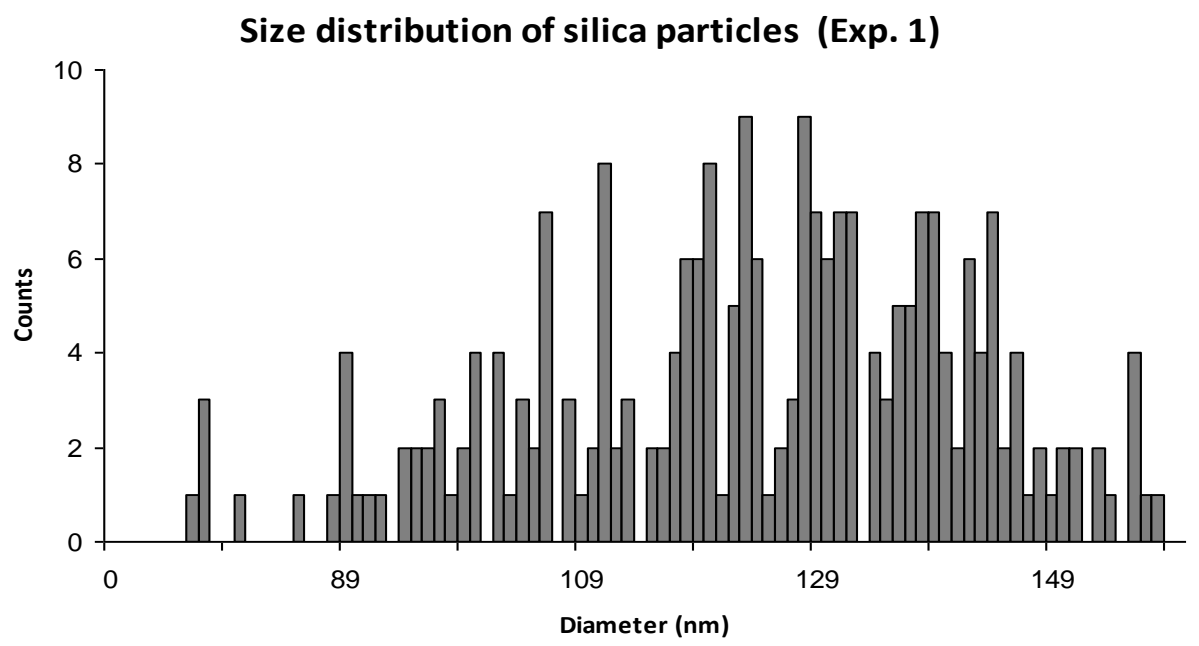

Figure 5.3: Size distribution of silica particles (Experiment 1). The distribution gives a mean value of $120 \mathrm{~nm} \pm 45 \mathrm{~nm}$. 
This histogram is created by analysing more than 200 particles. The histogram constructed in figure 5.3 shows that the silica particles have a mean diameter of approximately $120 \mathrm{~nm} \pm 45 \mathrm{~nm}$. This sample shows a standard deviation of more than $37 \%$ and this proves to be a very broad size distribution.

\section{Experiment 4 and experiment 5}

A more monodispersed sample is desired for the measurement of particle on qNano. Therefore, another method was employed to synthesize the silica particles. Both of experiment 4 and 5 differs from the method used in experiments 1-3 in terms of the order of mixing the solutions. Here, TEOS is mixed with a small volume of EtOH before adding to the ammonia-water-EtOH solution. The results are shown in figure 5.4.

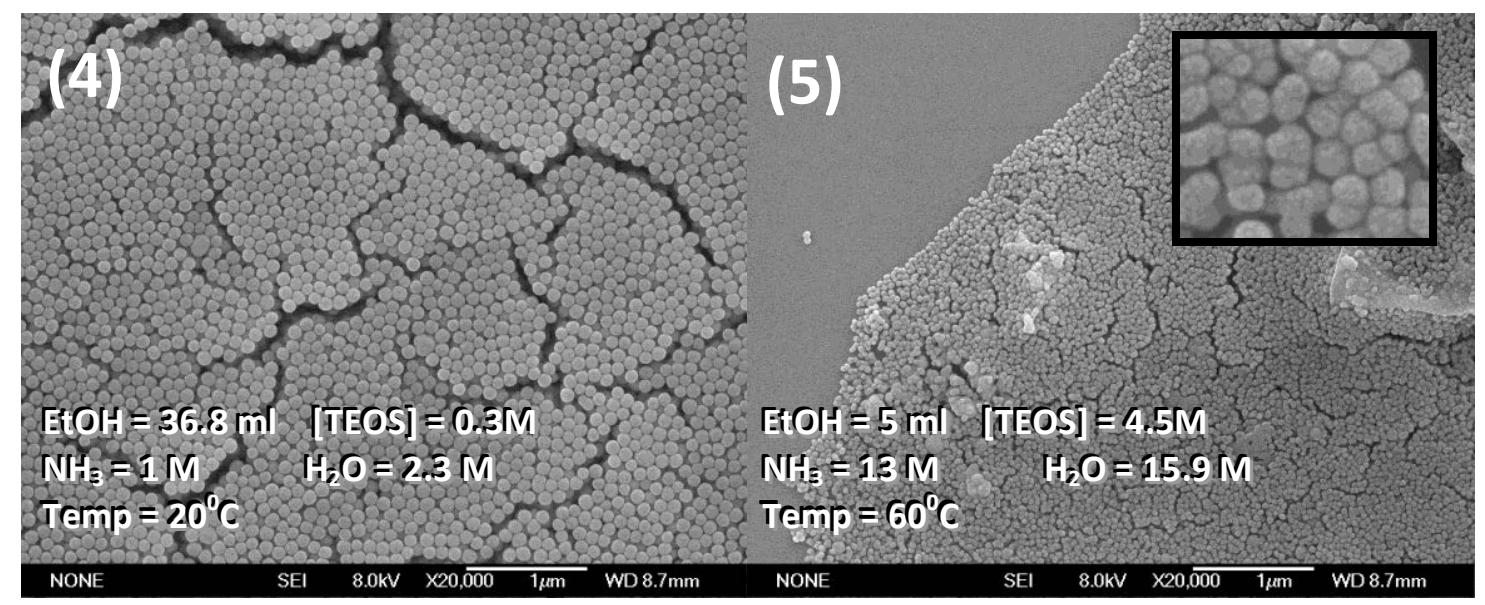

Figure 5.4: Images of silica particles at x20, 000 magnification. Sample from Experiment 5 shows small aggregates of silica particles.

Experiment 4 shows spherical silica particles that has a uniform dispersity. All of the reaction conditions of experiment 4 and experiment 5 were changed (except for the reaction time, which was kept constant at 6 hours). As seen in figure 5.4, the particles with a higher concentration of precursor shows aggregated particles (as seen in the inset). Due to the non-spherical particles synthesized in experiment 5, this sample will not be used in measurements on the qNano. The size distribution of sample 4 is given in figure 5.5. 


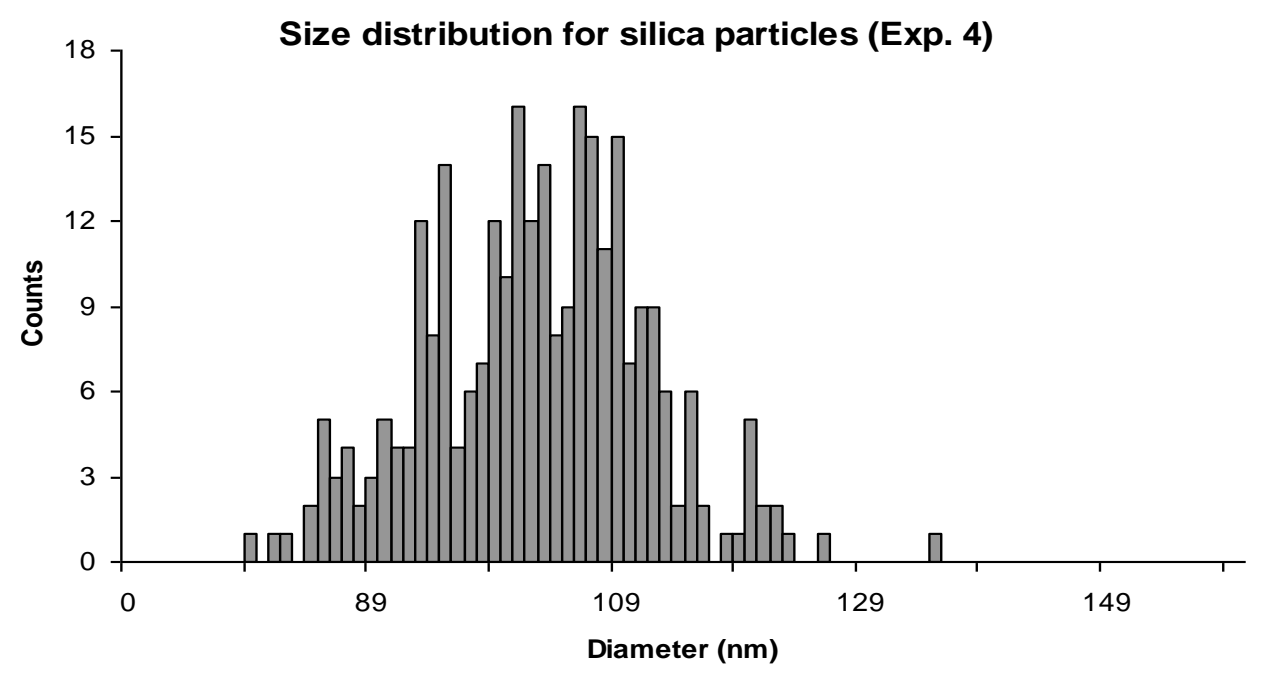

Figure 5.5: Size distribution of silica particles (Experiment 4). The distribution gives a mean value of $106 \mathrm{~nm} \pm 25 \mathrm{~nm}$.

Figure 5.5 shows the size distribution of experiment 4 . This histogram shows a tighter distribution of silica particles as compared to the distribution given in figure 5.3, confirming the visual assumption made about the distribution before. Sample 4 has a mean diameter of approximately $106 \mathrm{~nm} \pm 25 \mathrm{~nm}$, which is more monodispersed than samples in experiment 1.

\subsubsection{Synthesis discussion}

The experiments have shown that when the reaction is carried out with the concentration of ammonia more than $1 \mathrm{M}$, larger particles are produced such in sample 2. This is because ammonia is a catalyst and therefore increases the reaction rates. Previously, this has been observed by Lindberg et al. [70] and is attributed to the excess of hydroxide ions $\left(\mathrm{OH}^{-}\right)$which promotes the hydrolysis reaction [70, 71].

A reaction time of four hours such as those in experiment 3 also produces aggregation. This is most like due to reaction being incomplete as experiment 3 in figure 5.2 shows particles are joined together in a cluster that could lead to a formation of a larger and more spherical particles [70]. 
Another reason of cluster formation such as those in experiment 5 is due to more than $1 \mathrm{M}$ of water concentration, which increases its ionic conductivity strength during hydrolysis of TEOS and ammonia hydroxide $[46,68]$. As explained in chapter 4 , stability of nanoparticles in a solution depends on the thickness of the electrical double layer, a layer that electrostatically stabilizes nanoparticles. The thickness of the layer is inversely proportional to the concentration of ions in the solution. Therefore, an increase in concentration screens the electrostatic repulsion and induces particles flocculation [68].

\subsubsection{Synthesis conclusion}

It can be seen that experiment 1 and 4, which has a diameter of $120 \mathrm{~nm}$ and $106 \mathrm{~nm}$ respectively, is the most uniformly shaped spherical silica particles that can be used in measurements on qNano. Although the distributions are considered quite large, the particles are isolated (not aggregating) and this is important in order to have a good and stable measurements.

\subsubsection{Experimental methods}

As in the chapter 3 , specifically in section 3.2 .1 .2 , all measurements with silica particles were done in the presence of casein protein. Casein is a type of protein added to the particle and buffer solution mixture to give stability to the particles running through the nanopore. The experimental set-up is similar to that described in section 3.2.1.2.1 where blocking solutions (a solution with a high concentration of casein) were prepared to initially coat the aperture. Once the aperture is coated with the blocking solution, measurements of silica particles can be carried out. The silica particles are suspended in a casein-laced buffer solution of 1:1000 dilutions. A voltage bias of $0.18 \mathrm{~V}$ was used for all silica particles measurements.

\subsubsection{Results and Analysis}

The two sets of silica particles used - the $120 \mathrm{~nm}$ and $106 \mathrm{~nm}$ - differs only by $14 \mathrm{~nm}$. At this stage of the research, the IZON'S aperture has a limited ability to resolve difference of less than $100 \mathrm{~nm}$ in particle size. Therefore, the magnitude of the 
blockade events produced by both sets of particles was expected to be the same as can be seen in figure 5.6 .

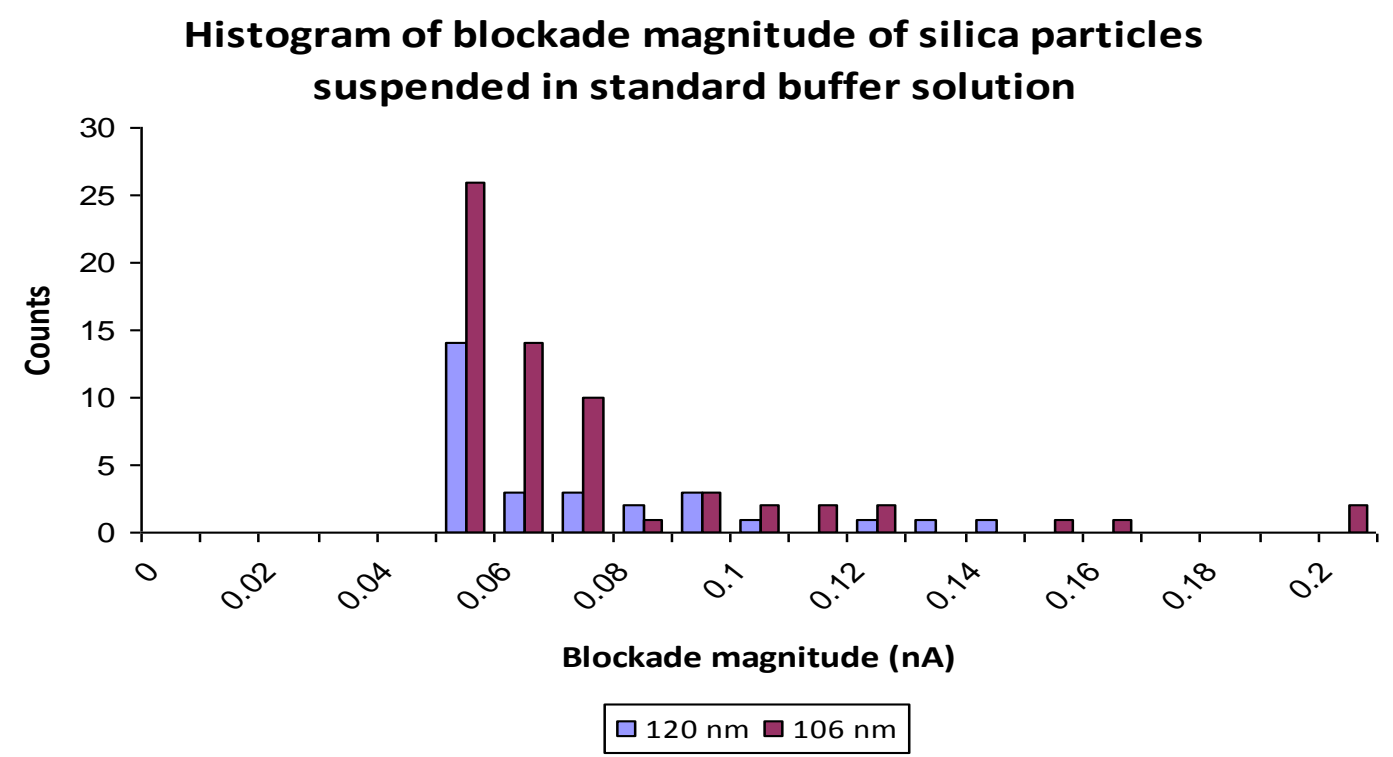

Figure 5.6: Distribution of silica particles suspended in IZON's standard buffer solution as detected by the qNano. Threshold of blockade magnitude was set at $0.05 \mathrm{nA}$ therefore only blockade with magnitude more than $0.05 \mathrm{nA}$ will be recorded.

Figure 5.6 shows the magnitude of blockade events of silica particles suspended in the buffer solution. Both of the particle sets shows that the majority of particle translocation has a blockade magnitude of $0.05 \mathrm{nA}$. Both signals appear the same as the aperture is unable to resolve two sets of particles that differ from each other by 14 $\mathrm{nm}$.

\subsubsection{Discussion}

There are a few issues to discuss when silica particles were passed through the nanopore. For most of the sample measured, the background current had issues with stability as well as high noise level. This observation occurs either almost immediately after silica particles were introduced to the upper fluid cell.

Much time was spent ensuring the silica particles were stable as they pass through the pore but no successful trial was achieved. Further investigation shows that it was due 
to the ethanol solvent used to make the silica particles that affect the stability given to the particles by the casein protein [66]. There are four types of casein, which are the $\alpha_{\mathrm{s} 1}, \alpha_{\mathrm{s} 2}, \beta$, and $\mathrm{k}$. Out of these, only the $\mathrm{k}$-casein shows ability to stabilize the micelle against further aggregation when there is a chemical change in the environment [22]. However, it has been reported that the addition of ethanol induces the coagulation of casein micelle $[22,66]$, which disintegrate its micellar form and retard its mobility in solution. Specifically, ethanol cleaves the micelle structure to form two parts: macropeptide that is strongly hydrophilic and para-k-casein, a hydrophobic part of the micelle. The ethanol collapses the outer 'hairy' layers, i.e. the macropeptide part of the casein micelle, hence renders the steric stabilization ineffective $[22,66]$. The maximum amount of ethanol that would give the casein laced buffer solution a stable baseline current was investigated and it was found that the minimal $5 \%$ ethanol content in the buffer solution was enough for erratic current to be observed. The erratic background current could also be due to the presence of silica aggregates.

The sizes of the silica nanoparticles were determined by the SEM images. Results from this measurement shows that the qNano cannot differentiate between the $120 \mathrm{~nm}$ and $104 \mathrm{~nm}$ silica nanoparticles. The inability to differentiate between the two sized nanoparticles is due to the limitation of the aperture to resolve particles that has a 14 $\mathrm{nm}$ difference in diameter. Further development to the apertures should look into making apertures that are rigid which in turn minimise aperture softening.

\subsection{Silver nanoparticles}

Silver nanoparticles were used to test the instrument's ability to measure noble metal particles. Like gold nanoparticles, silver nanoparticles are one of other noble metals that draw high attention due to its use in optics, electronics, catalysis and biomedical application. Therefore, having an instrument that is able to detect silver nanoparticles would open up more research interest in further characterising silver particles, which in turn would open up such a market for IZON. Here, brief investigation on silver nanoparticles is discussed. 


\subsubsection{Experimental methods}

The silver particles used in this experiment have a diameter of approximately $60 \mathrm{~nm}$. The particles were similar to the gold nanoparticles synthesised in chapter 4 i.e. using the citrate reduction method. The citrate group acts as a reducing agent as well as a stabilizer for the silver nanoparticles [78].

The silver nanoparticles were suspended in the standard buffer solution in a 1:166 dilution. IZON's $100 \mathrm{~nm}$ polystyrene nanoparticles were used in this experiment as a reference these were suspended in the buffer solution in a 1:1000 dilution. The running voltage used in this experiment was $0.3 \mathrm{~V}$.

\subsubsection{Results and Analysis}

Thus far, polystyrene nanoparticles have been used as a reference to determine the suitable aperture stretch. Since the measurement of silver nanoparticles has not been tested elsewhere, the signal profile has not been established. However, the experiments in this study have shown that the silver nanoparticles can be detected by its known diameter of $60 \mathrm{~nm}$. This value was determined by comparing the signal profile of the polystyrene nanoparticles and blockade events for both nanoparticles were recorded as given in figure 5.7. 
Distribution of blockade events for silver nanoparticles suspended in standard buffer solution

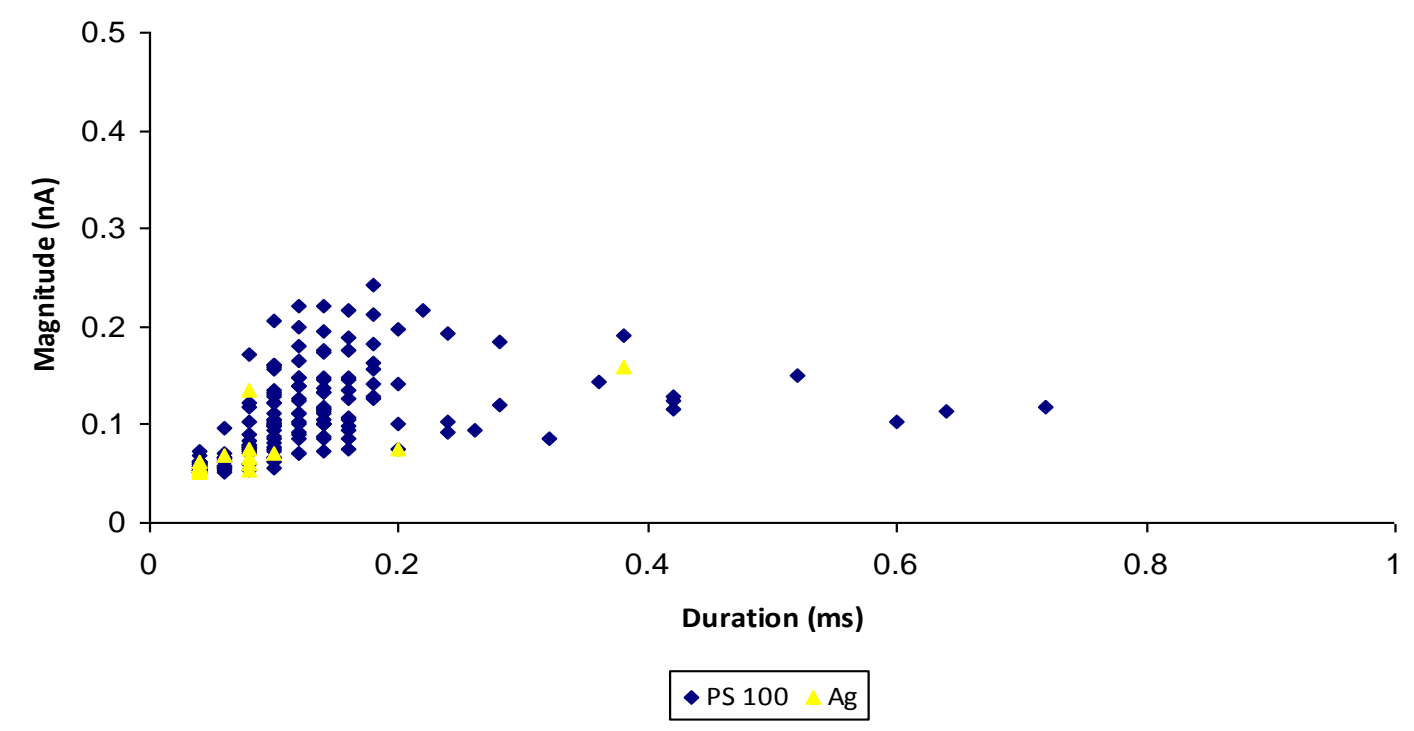

Figure 5.7: Blockade events of $100 \mathrm{~nm}$ polystyrene (PS) nanoparticles and silver nanoparticles suspended in the standard buffer solution. Blockade points from silver nanoparticles $(60 \mathrm{~nm})$ coincide with the signals from $100 \mathrm{~nm}$ polystyrene particles, which imply that it may have form aggregates in the solution.

Figure 5.7 shows a graph of blockade magnitude against blockade duration of silver and polystyrene nanoparticles. It is shown that silver nanoparticles were able to be detected as the data points from silver nanoparticles (in yellow) coincide with data points from $100 \mathrm{~nm}$ polystyrene (in blue). It can be seen that $100 \mathrm{~nm}$ polystyrene nanoparticles has an average blockade magnitude that spans from 0.05-0.2 nA, while the $60 \mathrm{~nm}$ silver nanoparticles has a blockade magnitude that covers from $0.05-0.08$ $n A$. This overlapping in data points suggest that the silver particles have grown to a diameter of the referenced polystyrene particles. This observation is also similar as seen in measurements for gold nanoparticles in section 4.3.1.

As discussed earlier, as resolvability potential of the aperture is limited, the range of stretch position overlaps. Hence, this makes identification of two nanoparticles of approximate sizes very challenging. At this stage, measurements from this research shows that the instrument can resolve two sized particles set that have a difference of approximately $400 \mathrm{~nm}$. The overlap of the particle sets stretch range means that 
blockade signals from each species of nanoparticles will mirror each other as shown in figure 5.8 .

\section{Histogram of blockade magnitudes of silver and polystyrene nanoparticles}

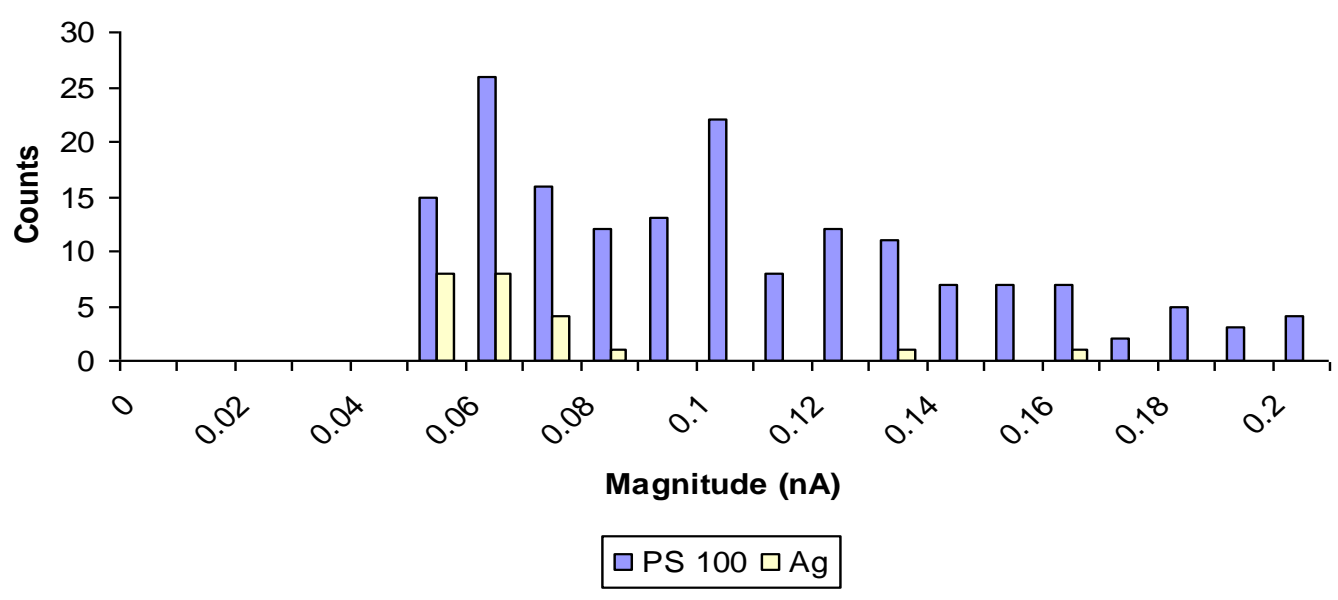

Figure 5.8: Distribution of blockade magnitudes of $60 \mathrm{~nm}$ silver nanoparticles and $100 \mathrm{~nm}$ polystyrene nanoparticles (PS) suspended in the standard buffer solution.

As shown in figure 5.8, particle blockades from silver nanoparticles give a mode of 0.05 $\mathrm{nA}$ while polystyrene particles give a mode of $0.07 \mathrm{nA}$. Characterisation using only the magnitude of the blockade is insufficient to distinguish between the two species of nanoparticles. Therefore, analysis using blockade duration was performed and the results are given in figure 5.9. 


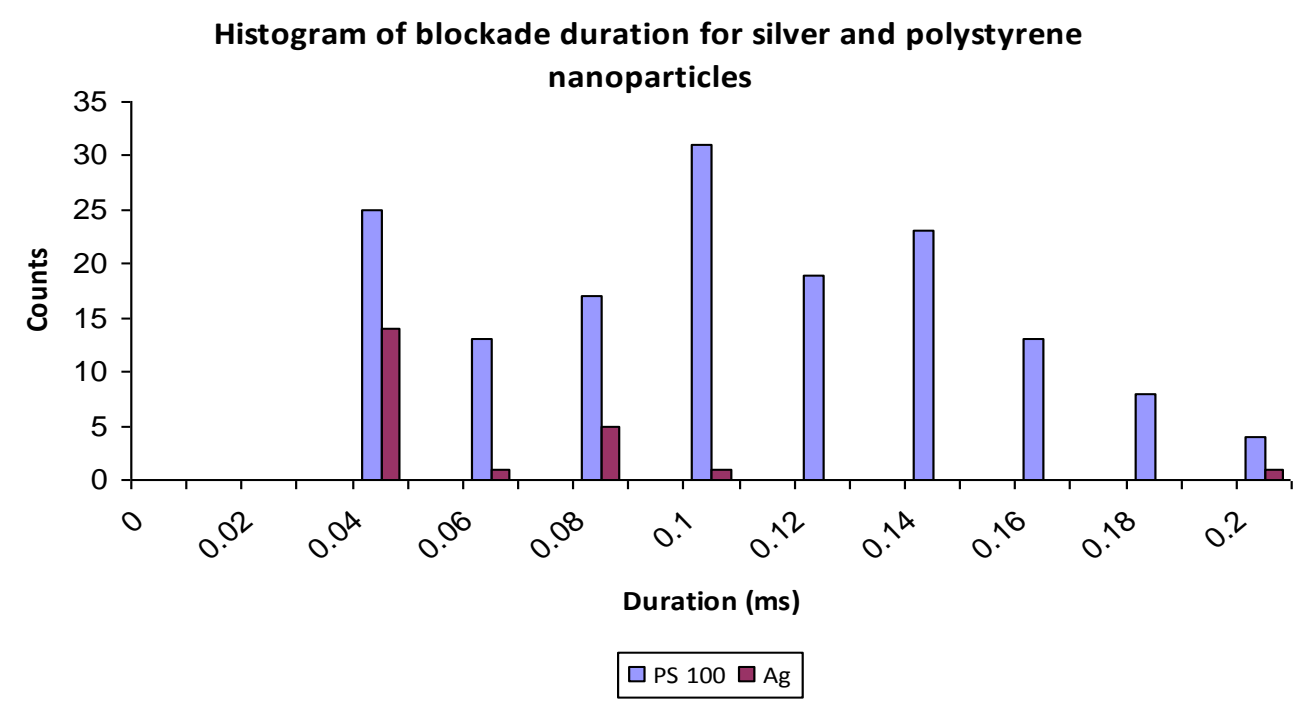

Figure 5.9: Distribution of blockade duration for $60 \mathrm{~nm}$ silver and $100 \mathrm{~nm}$ polystyrene (PS) nanoparticles suspended in the standard buffer solution. The histogram shows no noticeable difference of the translocation duration between the polystyrene and silver nanoparticles. The same pattern is also observed with gold nanoparticles discussed in chapter 4.

The results showed in figure 5.9 shows that majority of the detected $100 \mathrm{~nm}$ polystyrene nanoparticles goes through the pore at a translocation time of $0.1 \mathrm{~ms}$ while the silver nanoparticles goes through at a much faster rate of $0.04 \mathrm{~ms}$. From this result, it is assumed that identification of $60 \mathrm{~nm}$ silver nanoparticles can be made by just analysing the duration of translocated nanoparticles.

\subsubsection{Discussion}

It would appear that particle identification for silver nanoparticles is challenging as the silver and polystyrene nanoparticles show no major difference in the blockade magnitude. However, comparing the duration between silver and polystyrene particles suggests that the silver particles traverse through the pore at a much faster rate than polystyrene particles. Although measurements with silver particles capped with citrate ions were done several times, other methods of characterization needs to be provided to support and confirm this result. A different experimental measurement could also be designed to improve the detection of silver nanoparticles. This includes using larger 
diameter silver nanoparticles or using a different capping agent to the silver nanoparticles.

One trend that can be observed from this result is that magnitude signals from the silver nanoparticles approximate those of the referenced $100 \mathrm{~nm}$ polystyrene nanoparticles. Since the majority of the blockade magnitudes are similar to the ones produced by $100 \mathrm{~nm}$ polystyrene, it is assumed that silver nanoparticles also aggregate in the standard buffer solution as observed for the gold nanoparticles, in section 4.3. This experiment further supports the premise that citrate capped metal nanoparticles aggregates in the standard buffer solution provided by IZON. This is assumed to be due to the $\mathrm{KCl}$ salt present in the buffer solution as discussed in section 4.3.1.

Another point to state is that the distribution of silver nanoparticles in this measurement was clouded by the low count rate and in appropriate threshold positions. A refinement to the measurement settings would have given a better conclusion to the behaviour of the silver nanoparticles in the system.

\subsection{Magnetic nanoparticles}

Over the past decade, a lot of interest is paid to the research of magnetic nanoparticles especially in the field of biomedicine. Success at detecting magnetic nanoparticles with this technology would be of a great benefit as it could be used in biomedicine as a tool to measure concentration analysis. In this section, magnetic nanoparticles that were used were synthesized iron (II, III) oxide that is coated with a dextran; a chemical compound that will render the nanoparticles water-soluble.

\subsubsection{Experimental Methods}

The magnetic nanoparticles used in this research utilises iron oxide particle coated with oleic acid [76, 100]. The nanoparticles are hydrophobic due to the long carbon chain of the oleic acid. These nanoparticles were subjected to a ligand exchange with dextran to make it water soluble in order for it to successfully suspend in the standard buffer solution. Dextran was chosen as it shows biocompatibility especially in the use of magnetic resonance imaging (MRI) [77]. The nanoparticles were alkali treated with 
dextran as it forms a stronger bond to the nanoparticles (iron atoms on the core surface) due to the presence of the carboxyl $\left(\mathrm{COO}^{-}\right)$group [77].

For the ligand exchange, $25 \mathrm{ml}$ of deionised water was mixed with $0.625 \mathrm{~g}$ of sodium hydroxide $(\mathrm{NaOH})$ and $1.25 \mathrm{~g}$ of dextran. The mixture was sonicated together for an hour. This solution will be called Solution A.

The iron oxide nanoparticles that were going to be used were suspended in toluene. These nanoparticles were separated by re-dispersing in hexane and allowing to it to evaporate in a desiccator. Once all the liquid has been evaporated, $0.004 \mathrm{~g}$ of the nanoparticles were then weighed and re-disperse in methanol that has a dilution of 0.4 $\mathrm{mg} / \mathrm{ml}$. These mixes were then further sonicated for two hours. At the end of the sonication time, 3 drops of Solution A was added to the vessel containing the iron oxide nanoparticles which was then further sonicated for another 75 minutes. The sample was purified by magnetic separation i.e. using a strong magnet and has it by the side of the vessel and tipping out the supernatant. The nanoparticles were then redispersed in deionised water and are ready to be used.

The measurements of the magnetic nanoparticles were done at three different dilutions i.e. 1:1000, 1:100, and 1:10. These magnetic nanoparticles were suspended in the standard buffer solution. The nanoparticles used have a diameter of less than 10 $\mathrm{nm}$ so it is expected that no single particle events will be observed due to aperture limitation. The nanoparticles were left suspended in the buffer solution overnight to induce aggregation before measurements were taken.

The measurements were done at an increasing concentration i.e. 1:1000, then 1:100, and 1:10 to avoid residual contamination of the pore blockage. The voltage was set to run at $0.3 \mathrm{~V}$ and with a stretch range of $\Delta \mathrm{XY}=8-9 \mathrm{~mm}$. 


\subsubsection{Results and Analysis}

Blockade events detected on this measurement was of aggregates instead of single particle translocation. The distribution of blockade events of the magnetic nanoparticles at different concentration is given in figure 5.10.

\section{Distribution of magnetic particles suspended in buffer solution}

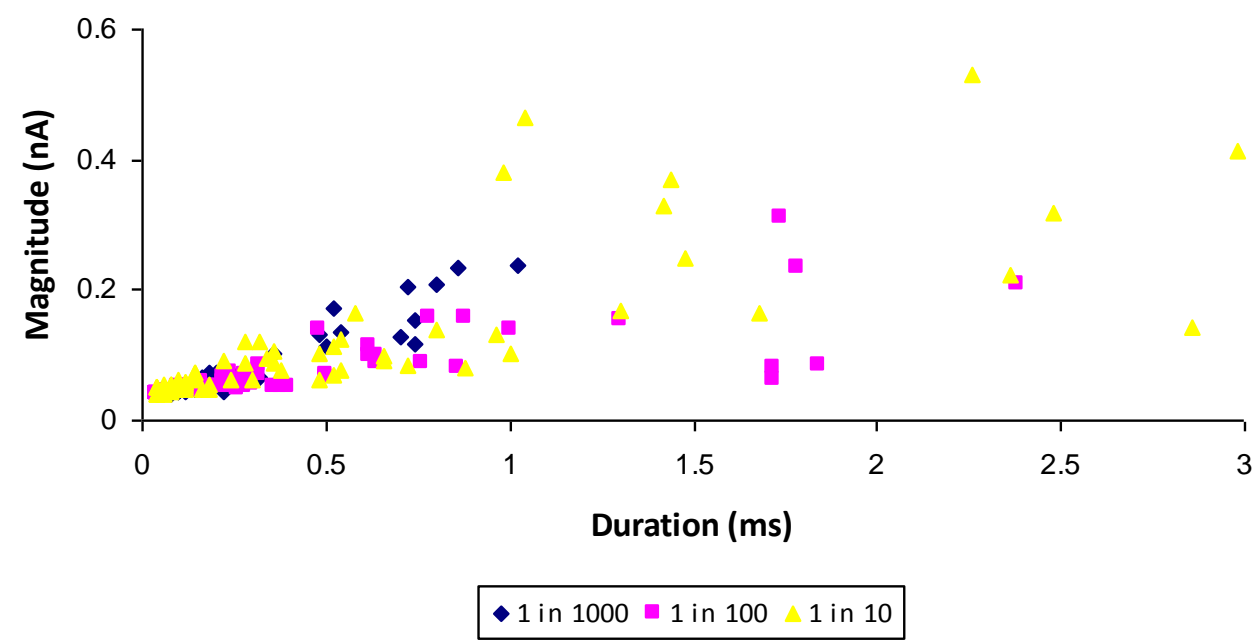

Figure 5.10: Distribution of magnetic nanoparticles at a different dilution suspended in IZON's standard electrolyte buffer. Larger aggregates formed as particle concentration is increased.

The distribution of the magnetic particle that was suspended in the buffer solution shows a similar trend to that of the gold nanoparticles in the same system discussed in section 4.3.5. In figure 5.10, it can be seen that $90 \%$ of the nanoparticles concentrate in the region that is at a magnitude between 0.03-0.18 nA and translocation duration of between $0.04-0.3 \mathrm{~ms}$. Results in figure 5.10 also shows that as the concentration of magnetic nanoparticles suspended in the buffer solution is increased, bigger blockades are detected as given by the magnitude of the blockades. This can be further substantiated with a plot of blockade magnitudes that quantify each blockade events detected as given in figure 5.11 . 


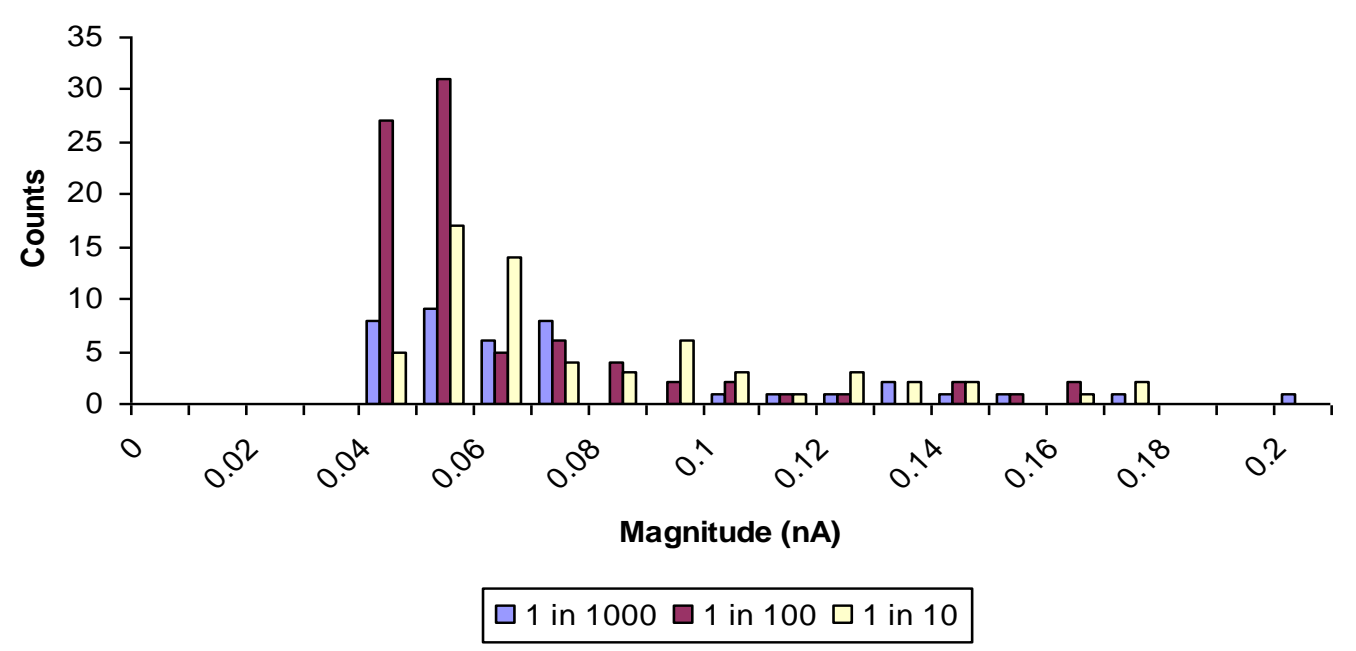

Figure 5.11: The distribution of magnetic nanoparticles at three different concentrations suspended in IZON's standard electrolyte buffer. Particles at all concentration have a blockade mode of $0.05 \mathrm{nA}$. Increase in concentration sees the increase in counts at larger magnitudes.

Figure 5.11 shows that blockades of nanoparticles in all dilution peaks at approximately $0.05 \mathrm{nA}$. The depth of the blockade magnitude reflects the diameter of nanoparticles that translocate through the aperture. Therefore, from figure 5.11, a higher concentration gives rise to higher blockade magnitude, which implies that the particle size increases as it is suspended in the buffer solution. Such trend was also observed with measurements of gold nanoparticles as discussed in section 4.3.5.2.

\subsubsection{Discussion}

As stated in the results section, blockade events that were detected in this measurement are suspected to be aggregates of magnetic nanoparticles instead of single nanoparticles. The measurements were carried out after the nanoparticles were suspended overnight as trials with freshly prepared sample give no blockades magnitude which supports the theory that the magnetic nanoparticles aggregates in the standard buffer solution. Figure 5.12 is the image taken of the sample of magnetic nanoparticles the day after the measurements were taken. 


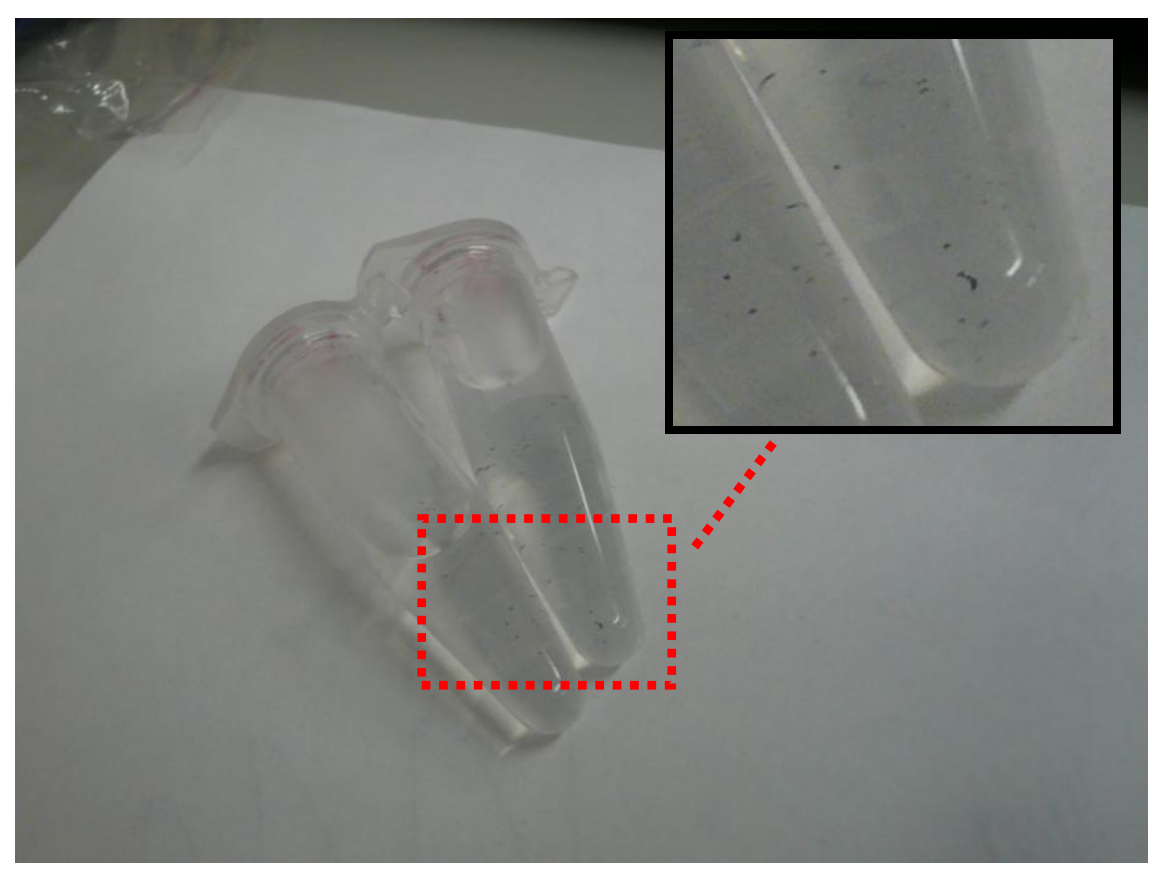

Figure 5.12: Image of magnetic nanoparticles aggregates suspended in IZON's standard buffer solution as seen with the naked eye.

As can be seen, there are aggregates of black iron oxide nanoparticles can be seen with the naked eye. This aggregates formed the day after the measurements were taken. This further confirms that the blockade events detected are of aggregates and not single particle events.

\subsection{Summary}

This chapter discuss the measurements of other types of nanoparticles on the qNano instrument. Section 5.2 looks into the measurement of silica particles. Silica nanoparticles were synthesized with a range of particles diameters. Two sizes of silica particles were used in measurements with qNano, which are $120 \mathrm{~nm}$ and $104 \mathrm{~nm}$ particles. Results from the measurements shows that the instrument is able to detect silica particles. However, qNano is unable to differentiate between the two diameters of silica particles due to factors that include possible aggregation of the silica particles, system stability that could be due to the addition of casein to the buffer system as well as the limitation of the aperture to resolve a size difference of less than $100 \mathrm{~nm}$. The silica particles were measured in the presence of casein molecules to aid particles stability as erratic current was observed during the measurements. This is explained by 
the presence of ethanol used in synthesizing the silica nanoparticles, which collapse the structure of the casein micelle. Section 5.3 then investigates the measurements of citrate reduced silver nanoparticles. It was found that the instrument is able to measure silver nanoparticles. However, due to the limitation of the aperture to resolve nanoparticles that has a small size difference, the range of stretch positions between polystyrene and silver nanoparticles overlaps. Section 5.4 looked at the instrument's ability to detect magnetic nanoparticles. The measurements however were of aggregates of magnetic nanoparticles and not of single particle events. 


\subsection{CONCLUSION}

This thesis has discussed the principal of IZON's qNano technology, which uses the Scanning Ion Occlusion Spectroscopy (SIOS) alongside an adjustable nanopore to detect inorganic and metal nanoparticles. The nanoparticles investigated in this research include polystyrene, gold, silver, silica, and magnetic nanoparticles. Polystyrene particles supplied by IZON were used extensively especially as a default test particles to investigate the workings of the qNano as well as a reference for the inorganic and metal nanoparticles.

With the SIOS technology, the usual driving force for particle translocation through the membrane is electrophoresis. Electrophoresis utilise the surface charge of the particles suspended in the buffer solution to move through the nanopore as an electric field is applied across the electrodes. All the particles measured in this research mainly used electrophoresis in aiding its translocation through the nanopore. However, throughout this research, IZON has done many upgrades to its technology that aids the detection of these nanoparticles. These changes consist of manufacturing smaller sized nanopore, and increasing the bandwidth as well as the development of the variable pressure module (VPM) as a supplementary mode of the driving force for particle translocation through the aperture. The VPM is especially beneficial for particles with an infinitesimal or non-existent surface charge. Running conditions such as the aperture, duration threshold as well as the amount of pressure used has to be tuned in order to get good data and results so that the workings of the system with the VPM can be better understood.

Understanding the operation and the ability of IZON's qNano to measure particles down to the nano region was the focus in Chapter 3. This includes discovering the capability of the qNano in resolving particles of different diameter - the bimodal distribution- and surface charge, as well as understanding the behaviour of IZON's aperture. The capability and sensitivity of the resizable nanopore manufactured by IZON has been established using both polystyrene particles as well as carboxylated 
polystyrene particles. Both of these particles are used as a standard calibration tool as it is one of the easier systems to understand.

Experiments conducted and explained in section 3.2.1 were done in order to characterise the aperture and understand its behaviour. Results from these experiments illustrate that the apertures have the tendency to exhibit material softening as an aperture is reused over time. The aperture exhibits Mullins effect, which is the phenomenon observed for the TPU aperture where the stress-strain curve is reproducible after repeated stress-cycling provided that it is not stretched past its maximum stretch limit. Straining the aperture above the maximum limit will cause irreversible softening and cause the sensitivity of the resizable aperture to decrease. Apart from softening of the aperture, it has also been shown that there is a lower and upper limit of workable aperture stretch where particle blockades will be registered. This range of stretch positions is typically found by stretching the aperture to its maximum stretch and particles' scanning is done as the pore is closed down. It has been found that it is better to close the pore down rather than stretching it open when optimising stretch for the particles to avoid blockage of the pore. Within this stretch range, there is a point that gives the most particles blockade counts. Providing that the correct threshold is set for each measurement, recognizing where this stretch lies could be of importance in particle concentration studies for example which can be explored in further research.

The instrument's ability to give a bimodal distribution proved that this technology is able to resolve different-sized particles in the same solution, as shown in section 3.2.2. This is a particularly useful feature to have especially for usage in size separation of particles or to establish the dispersity of an unknown sample. One of the limitation of this technology however is the ability for the aperture to resolve particles that have a small size separation e.g. to resolve between $200 \mathrm{~nm}$ and $250 \mathrm{~nm}$. This ability to resolve different sizes particles depends mostly on the sensitivity of the aperture to react to the stretching applied. However, due to softening of the tuneable nanopore this limits the aperture's sensitivity. 
The instrument was also tested to see if there is a difference in signals from polystyrene particles and carboxylated particles as discussed in section 3.2.3. The results for this shows that carboxylated particles travel through the pore slower than the polystyrene particles. This is contrary to the expected results due to the change in pore stretch during measurements. Increase in the pore stretch causes membrane thinning where the length of the nanopore is reduced by approximately $14 \%$ [97]. The electrophoretic mobility increases to the squared of the length of the nanopore. Therefore, a reduction in the pore length means that the electrophoretic mobility decreases to the power of two and hence an increase in the translocation duration. Results from section 3.2.3 shows that a constant aperture stretch is crucial for data comparisons between each sample set.

The last experiments in chapter 3 as explained in section 3.3 involve the use of polystyrene nanoparticles. These particles can be detected because of the increase in the instrument's bandwidth from a $10 \mathrm{kHz}$ to a $50 \mathrm{kHz}$. This upgrade in technology allows more data points to be recorded hence the ability to accurately detect nanoparticles which travel through the pore much faster than those that have a diameter of $800 \mathrm{~nm}$ for example [13]. Proof of the instrument to detect nanoparticles is vital as this opens up an area of research where this technology can be used to characterise metal and other nanoparticles.

Chapter 4 was dedicated to the measurement of gold nanoparticles with the qNano technology. The gold nanoparticles detected however, are believed to be aggregates and not of single nanoparticles like the polystyrene nanoparticles discussed in chapter 3. The results show that potassium chloride $(\mathrm{KCl})$ salt that is present in IZON's standard buffer solution is the main reason of the aggregation of gold nanoparticles. It was also found that aggregation of gold nanoparticles does not occur at a high rate, as detection of gold aggregates was only obtainable after a period of three hours of measurement time.

Detection of gold aggregates also increases as the concentration of gold suspended in the standard buffer solution increases. The mechanism of gold nanoparticles' 
aggregation in IZON's standard buffer solution possibly follows the same route for seeded growth of gold nanoparticles but this hypothesis is yet to be established. However, the actual mechanism for gold aggregation in IZON's standard buffer solution is not yet understood at this stage of the research and further investigation could be conducted.

On using this instrument to identify two species of nanoparticles, the instrument is unable to recognise different sets of nanoparticles but this could possibly be due to the measurement settings. Further exploration of data manipulation would be useful in order to distinguish the two types of nanoparticles present in the same solution.

With the use of the pressure system, it seems that this effect of pressure overcomes the effect of electrophoresis especially for the gold nanoparticles. Fewer blockade events of gold nanoparticles were detected under pure electrophoresis. However, with the addition of the pressure system, significant signals were detected. Discussions with collaborators showed that a substantial amount of counts has been detected with gold nanoparticles with the addition of bovine serum albumin (BSA) at a pressure of $1.2 \mathrm{kPa}$ [89]. This is more than twice the pressure used in this experiment so further investigation can be performed by changing the pressure parameter to see if there are any major effect on the blockade counts.

There is a lot of potential for the instrument to be used in characterising noble metal nanoparticles. As shown in chapter 5, the instrument was able to detect silica, silver and magnetic nanoparticles. Erratic current readings were observed during the measurement with silica nanoparticles. In addition, the nanoparticles experience instability when running through the aperture due to ethanol (from the solvent in synthesizing silica) collapsing the casein micelle structure which gives stability to the particles in the buffer system. There are other possible reasons that could contribute to the current instability as well which include the aperture being too small for the particles used or that there is blockage of the nanopore by the silica particles. 
Silver nanoparticles synthesized by the citrate reduction method show a degree of nanoparticles aggregation as seen with the gold nanoparticles in chapter 4 . This further confirms that the aggregation is caused by the interaction of the capping layer with the salt ions present in the buffer solution. In this case, $60 \mathrm{~nm}$ silver particles aggregate to approximately $100 \mathrm{~nm}$ in roughly two hours before measurement of silver nanoparticles were taken on the qNano. This also suggests that the extent of nanoparticle aggregation is a function of time.

The last section in chapter 5 looks into measurements of magnetic particles. Here, aggregation of magnetic particles also occurred although the reason is not yet understood. Different concentrations of magnetic particles were studied and it was found that the size of aggregated particles increases as the particle concentration increases. Future work could involve the study of the rate of aggregation of magnetic nanoparticles in the standard buffer solution.

Overall, this thesis has discussed and fulfilled the research objectives that were set in section 1.3. Inorganic and metal nanoparticles have been detected on IZON's qNano and the instrument has proved to be a valuable instrument in characterising nanoparticles especially in terms of particle sizing. The behaviour of the TPU aperture is also now better understood and the choice of the aperture used in future measurements can assist in obtaining a reproducible measurements. On top of that, this thesis illustrates the instrument's sensitivity towards external electromagnetic radiation that causes background current noise. Having the knowledge on how to prevent these unwanted noise signals will help future users in acquiring the optimal running conditions for their measurements.

\subsection{Future work}

As discussed in this thesis, metal nanoparticles aggregates when they are suspended in IZON's buffer solution. Further work could investigate how to stabilize metal nanoparticles from further aggregation in IZON's buffer solution. This could include changing the buffer solution of adding stabilizing agent to the buffer such as the measurements done with polystyrene particles stabilized with casein. 
Another interesting research that could be conducted is to use qNano instrument to identify two different species of particles in the same solution. The results obtained from the measurements done in this research shows that the qNano instrument is unable to resolve two different species of particles. Further research in finding the workable measurement settings to resolve the different particles would be useful especially in the field of particle separation.

As discussed in chapter 2 and 4, the apertures have a limitation in resolving two sized nanoparticles that have a difference of less than $100 \mathrm{~nm}$. The limitation is due to the size of the nanopore (determined upon fabrication) as well as softening of aperture over use. Further research could look into the minimal size difference that the aperture can resolve. Following from that, further development of apertures should look into fabricating apertures that are more rigid, which could minimise the softening effect. This should hopefully enable the qNano instrument to resolve two sized nanoparticles with a difference of less than $100 \mathrm{~nm}$.

One of the most interesting aspects for further research opportunities regarding measurements on the qNano technology is the interaction between all the particles studied in this research and the IZON's standard buffer solution that was reported in chapter 4 and 5. Numerous investigations are possible which further proves to show that IZON's qNano instrument is a valuable research instrument for the characterization of nanoparticles. 


\section{REFERENCES}

1) Li, J. L., M. Gershow, et al. (2003). "DNA molecules and configurations in a solidstate nanopore microscope." Nature Materials 2(9): 611-615.

2) Mara, A., Z. Siwy, et al. (2004). "An asymmetric polymer nanopore for single molecule detection." Nano Letters 4(3): 497-501.

3) Saleh, O. A. and L. L. Sohn (2001). "Quantitative sensing of nanoscale colloids using a microchip Coulter counter." Review of Scientific Instruments 72(12): $4449-4451$

4) Deamer, D. W. and D. Branton (2002). "Characterization of nucleic acids by nanopore analysis." Accounts of Chemical Research 35(10): 817-825.

5) Ito, T., L. Sun, et al. (2003). "Simultaneous Determination of the Size and Surface Charge of Individual Nanoparticles Using a Carbon Nanotube-Based Coulter Counter." Analytical Chemistry 75(10): 2399-2406.

6) Sowerby, S. J., M. F. Broom, et al. (2007). "Dynamically resizable nanometrescale apertures for molecular sensing." Sensors and Actuators B-Chemical 123(1): 325-330.

7) Kasianowicz, J. J., E. Brandin, et al. (1996). "Characterization of individual polynucleotide molecules using a membrane channel." Proceedings of the National Academy of Sciences of the United States of America 93(24): 1377013773.

8) Bayley, H. and P. S. Cremer (2001). "Stochastic sensors inspired by biology." Nature 413(6852): 226-230.

9) Fortina, P., L. J. Kricka, et al. (2005). "Nanobiotechnology: the promise and reality of new approaches to molecular recognition." Trends in Biotechnology 23(4): 168-173.

10) Bayley, H. and C. R. Martin (2000). "Resistive-pulse sensing - From microbes to molecules." Chemical Reviews 100(7): 2575-2594.

11) Yu, S. Personal Communications. October 2009.

12) Arnold, W.M. (17 April 2009). "Electrical manipulation of particles within a nanopore".

< http://www.electrostaticanswers.com/2009ESJC/Poster1/2009\%20Arnold\%20 
Electrical\%20Manipulation\%20of\%20Particles\%20with\%20a\%20Nanopore.pdf> 29 May 2009.

13) Uram, J. D., K. Ke, et al. (2008). "Noise and bandwidth of current recordings from sub micrometer pores and nanopores." Acs Nano 2(5): 857-872.

14) Jagtiani, A. V., J. Zhe, et al. (2006). "Detection and counting of micro-scale particles and pollen using a multi-aperture Coulter counter." Measurement Science \& Technology 17(7): 1706-1714.

15) Smeets, R. M. M., S. W. Kowalczyk, et al. (2009). "Translocation of RecA-Coated Double-Stranded DNA through Solid-State Nanopores." Nano Letters 9(9): 3089-3095.

16) Noordman, T. R. and J. A. Wesselingh (2002). "Transport of large molecules through membranes with narrow pores: The Maxwell-Stefan description combined with hydrodynamic theory." Journal of Membrane Science 210(2): 227-243.

17) Henriquez, R. R., T. Ito, et al. (2004). "The resurgence of Coulter counting for analyzing nanoscale objects." Analyst 129: 478-482.

18) Wang, P. H. and C. Y. Pan (2000). "Polymer-metal composite particles: Metal particles on poly (St-co-MAA) microspheres." Journal of Applied Polymer Science 75(14): 1693-1698.

19) Wang, P. H. and C. Y. Pan (2000). "Preparation of styrene/methacrylic acid copolymer microspheres and their composites with metal particles." Colloid and Polymer Science 278(6): 581-586.

20) Wang, P. H. and C. Y. Pan (2002). "Preparation of styrene/acrylic acid copolymer microspheres: polymerization mechanism and carboxyl group distribution." Colloid and Polymer Science 280(2): 152-159.

21) Yan, Y. and Q. M. Chen (2009). "Rapid Synthesis of Monodisperse Polystyrene Microspheres and Self-Assembly of Colloidal Crystals on Mica." Journal of Dispersion Science and Technology 30(4): 575-580.

22) Holt, C. and D. G. Dalgleish (1986). "Electrophoretic and hydrodynamic properties of bovine casein micelles interpreted in terms of particles with an outer hairy layer." Journal of Colloid and Interface Science 114(2): 513-524. 
23) Zhu, J. and P. Li (2003). "Synthesis and Characterization of Poly (methyl methacrylate)/Casein Nanoparticles with a Well-Defined Core-Shell Structure." Journal of Polymer Science, Part A: Polymer Chemistry 41(21): 3346-3353.

24) Anema, S. G. "The effect of chymosin on [kappa]-casein-coated polystyrene latex particles and bovine casein micelles." International Dairy Journal 7(8-9): 553-558.

25) Dalgleish, D. G., E. Dickinson, et al. (1985). "Ionic-strength effects on the electrophoretic mobility of casein-coated polystyrene latex-particles." Journal of Colloid and Interface Science 108(1): 174-179.

26) Muthukumar, M. (1999). "Polymer translocation through a hole." Journal of Chemical Physics 111(22): 10371-10374.

27) He, Y.-D., H.-J. Qian, et al. (2007). "Polymer translocation through a nanopore in mesoscopic simulations." Polymer 48(12): 3601-3606.

28) Hui, L., Q. Shizhi, et al. (2007). "The effect of translocating cylindrical particles on the ionic current through a nanopore." Biophysical Journal 92(4): 1164-77.

29) Slonkina, E. and A. B. Kolomeisky (2003). "Polymer translocation through a long nanopore." Journal of Chemical Physics 118(15): 7112-7118.

30) Kolomeisky, A. B. (2008). "How polymers translocate through pores: Memory is important." Biophysical Journal 94(5): 1547-1548.

31) Luo, M.-B. (2007). "Translocation of polymer chains through interacting nanopores." Polymer 48(26): 7679-7686

32) Martin, C. R., M. Nishizawa, et al. (2001). "Investigations of the transport properties of gold nanotubule membranes." Journal of Physical Chemistry B 105(10): 1925-1934.

33) Lansac, Y., P. K. Maiti, et al. (2004). "Coarse-grained simulation of polymer translocation through an artificial nanopore." Polymer 45(9): 3099-3110.

34) Willmott, G. R., R. Vogel, et al. "Use of tuneable nanopore blockade rates to investigate colloidal dispersions." Journal of Physics-Condensed Matter 22(45): 11.

35) Jana, N. R., L. Gearheart, et al. (2001). "Seeding growth for size control of 5-40 nm diameter gold nanoparticles." Langmuir 17(22): 6782-6786. 
36) Pesika N.S, Stebe K.J., et al. (2003). "Relationship between Absorbance Spectra and Particle Size Distributions for Quantum-Sized Nanocrystals." Journal of Physical Chemistry B 107(38): 10412-10415.

37) Turkevich, J., P. C. Stevenson, et al. (1951). "A STUDY OF THE NUCLEATION AND GROWTH PROCESSES IN THE SYNTHESIS OF COLLOIDAL GOLD." Discussions of the Faraday Society (11): 55-\&.

38) Grabar, K. C., R. G. Freeman, et al. (1995). "PREPARATION AND CHARACTERIZATION OF AU COLLOID MONOLAYERS." Analytical Chemistry 67(4): 735-743.

39) Ji, X., X. Song, et al. (2007). "Size Control of Gold Nanocrystals in Citrate Reduction: The Third Role of Citrate." Journal of the American Chemical Society 129(45): 13939-13948.

40) Brown, K. R., D. G. Walter, et al. (2000). "Seeding of colloidal Au nanoparticle solutions. 2. Improved control of particle size and shape." Chemistry of Materials 12(2): 306-313.

41) Zhou, J., J. Ralston, et al. (2009). "Functionalized gold nanoparticles: Synthesis, structure and colloid stability." Journal of Colloid and Interface Science 331(2): 251-262.

42) Kimling, J., M. Maier, et al. (2006). "Turkevich method for gold nanoparticle synthesis revisited." Journal of Physical Chemistry B 110(32): 15700-15707.

43) Perrault, S. D. and W. C. W. Chan (2009). "Synthesis and Surface Modification of Highly Monodispersed, Spherical Gold Nanoparticles of 50-200 nm." Journal of the American Chemical Society 131(47): 17042-17043.

44) Levy, R., N. T. K. Thanh, et al. (2004). "Rational and combinatorial design of peptide capping Ligands for gold nanoparticles." Journal of the American Chemical Society 126(32): 10076-10084.

45) Gu, S., J. Onishi, et al. (2004). "Preparation of multilayered gold-silicapolystyrene core-shell particles by seeded polymerization." Journal of Colloid and Interface Science 279(1): 284-287.

46) Mine, E., A. Yamada, et al. (2003). "Direct coating of gold nanoparticles with silica by a seeded polymerization technique." Journal of Colloid and Interface Science 264(2): 385-390. 
47) Lee, H. B., Y. M. Yoo, et al. (2006). "Characteristic optical properties and synthesis of gold-silica core-shell colloids." Scripta Materialia 55(12): 11271129.

48) Philip, D. (2008). "Synthesis and spectroscopic characterization of gold nanoparticles." Spectrochimica Acta Part A: Molecular and Biomolecular Spectroscopy 71(1): 80-85.

49) Sugunan, A., C. Thanachayanont, et al. "Heavy-metal ion sensors using chitosan-capped gold nanoparticles." Science and Technology of Advanced Materials 6(3-4): 335-340.

50) Liu, Y. and R. Guo (2009). "The interaction between casein micelles and gold nanoparticles." J Colloid Interface Sci 332(1): 265-9.

51) Perrault, S. D. and W. C. W. Chan (2009). "Synthesis and Surface Modification of Highly Monodispersed, Spherical Gold Nanoparticles of 50-200 nm." Journal of the American Chemical Society 131(47): 17042-17043.

52) Stoeva, S. I., A. B. Smetana, et al. (2007). "Gram-scale synthesis of aqueous gold colloids stabilized by various ligands." Journal of Colloid and Interface Science 309(1): 94-98.

53) Daniel, M. C. and D. Astruc (2004). "Gold nanoparticles: Assembly, supramolecular chemistry, quantum-size-related properties, and applications toward biology, catalysis, and nanotechnology." Chemical Reviews 104(1): 293346.

54) Yang, Y., S. Matsubara, et al. (2007). "Controlling the aggregation behaviour of gold nanoparticles." Materials Science and Engineering: B 140(3): 172-176.

55) Pashley, R. M. and M. E. Karaman (2005). Van der Waals Forces and Colloid Stability, John Wiley \& Sons, Ltd.

56) Qian, S. Z. and S. W. Joo (2008). "Analysis of self-electrophoretic motion of a spherical particle in a nanotube: Effect of nonuniform surface charge density." Langmuir 24(9): 4778-4784.

57) Norman, T. J., C. D. Grant, et al. (2002). "Near infrared optical absorption of gold nanoparticle aggregates." Journal of Physical Chemistry B 106(28): 70057012. 
58) Smitha, S. L., K. M. Nissamudeen, et al. (2008). "Studies on surface plasmon resonance and photoluminescence of silver nanoparticles." Spectrochimica Acta Part A: Molecular and Biomolecular Spectroscopy 71(1): 186-190.

59) Gang, W. and S. Wenfang (2006). "Optical limiting of gold nanoparticle aggregates induced by electrolytes." Journal of Physical Chemistry B 110(42): 20901-5.

60) Ojea-Jimenez, I. and V. Puntes "Instability of Cationic Gold Nanoparticle Bioconjugates: The Role of Citrate Ions (vol 131, pg 13320, 2009)." Journal of the American Chemical Society 132(14): 5322-5322

61) Kim, S. M., G. S. Kim, et al. (2008). "Effects of PVP and $\mathrm{KCl}$ concentrations on the synthesis of gold nanoparticles using a solution plasma processing." Materials Letters 62(28): 4354-4356.

62) Patungwasa, W. and J. H. Hodak (2008). "pH tuneable morphology of the gold nanoparticles produced by citrate reduction." Materials Chemistry and Physics 108(1): 45-54.

63) Guan, J., L. Jiang, et al. (2008). "pH-dependent response of citrate capped Au nanoparticle to $\mathrm{Pb} 2+$ ion." Colloids and Surfaces A: Physicochemical and Engineering Aspects 325(3): 194-197.

64) Sylvestre, J. P., S. Poulin, et al. (2004). "Surface chemistry of gold nanoparticles produced by laser ablation in aqueous media." Journal of Physical Chemistry B 108(43): 16864-16869.

65) Munro, C. Core-Shell Nanocrystals for Synthetic Opal. Diss. Victoria University of Wellington, 2005. Wellington: n/a. 2005.

66) Horne, D. S. (1984). "STERIC EFFECTS IN THE COAGULATION OF CASEIN MICELLES BY ETHANOL." Biopolymers 23(6): 989-993.

67) Lubensky, D. K. and D. R. Nelson (1999). "Driven polymer translocation through a narrow pore." Biophysical Journal 77(4): 1824-1838.

68) Liz-Marzán, L. M. and A. P. Philipse (1995). "Synthesis and Optical Properties of Gold-Labelled Silica Particles." Journal of Colloid and Interface Science 176(2): 459-466.

69) Lu, Y., Y. Yin, et al. (2002). "Synthesis and Self-Assembly of Au@SiO2 Core-Shell Colloids." Nano Letters 2(7): 785-788. 
70) Lindberg, R., G. r. Sundholm, et al. (1997). "Multivariate analysis of the size dependence of monodisperse silica particles prepared according to the sol-gel technique." Colloids and Surfaces A: Physicochemical and Engineering Aspects 123-124: 549-560.

71) Matsoukas, T. and E. Gulari (1988). "Dynamics of growth of silica particles from ammonia-catalyzed hydrolysis of tetra-ethyl-orthosilicate." Journal of Colloid and Interface Science 124(1): 252-261.

72) Giesche, H. (1994). "SYNTHESIS OF MONODISPERSED SILICA POWDERS .1. PARTICLE PROPERTIES AND REACTION-KINETICS." Journal of the European Ceramic Society 14(3): 189-204.

73) Bogush, G. H. and C. F. Zukoski Iv (1991). "Studies of the kinetics of the precipitation of uniform silica particles through the hydrolysis and condensation of silicon alkoxides." Journal of Colloid and Interface Science 142(1): 1-18.

74) Bogush, G. H. and C. F. Zukoski Iv (1991). "Uniform silica particle precipitation: An aggregative growth model." Journal of Colloid and Interface Science 142(1): 19-34.

75) Bogush, G. H., M. A. Tracy, et al. (1988). "Preparation of monodisperse silica particles: Control of size and mass fraction." Journal of Non-Crystalline Solids 104(1): 95-106.

76) Chang, S. M., M. Lee, et al. (2005). "Preparation of large monodispersed spherical silica particles using seed particle growth." Journal of Colloid and Interface Science 286(2): 536-542.

77) Kawaguchi, T. and M. Hasegawa (2000). "Structure of dextran-magnetite complex: relation between conformation of dextran chains covering core and its molecular weight." Journal of Materials Science-Materials in Medicine 11(1): 31-35.

78) Henglein, A. and M. Giersig (1999). "Formation of colloidal silver nanoparticles: Capping action of citrate." Journal of Physical Chemistry B 103(44): 9533-9539.

79) Le Ru, Eric. Personal Interview. 27 April 2009.

80) Qi, H. J. and M. C. Boyce (2005). "Stress-strain behaviour of thermoplastic polyurethanes." Mechanics of Materials 37(8): 817-839. 
81) Buckley, C. P., C. Prisacariu, et al. "Elasticity and inelasticity of thermoplastic polyurethane elastomers: Sensitivity to chemical and physical structure." Polymer 51(14): 3213-3224.

82) Trabelsi, S., P. A. Albouy, et al. (2003). "Effective Local Deformation in Stretched Filled Rubber." Macromolecules 36(24): 9093-9099.

83) Mullins, L. (1969). Rubber Chem. Technol., 42, 339

84) Diani, J., B. Fayolle, et al. (2009). "A review on the Mullins effect." European Polymer Journal 45(3): 601-612.

85) Mullins L, Tobin NR. Theoretical model for the elastic behaviour of filler reinforced vulcanized rubbers. Rubber Chem Technol 1957; 30:555-71.

86) Willmott, G. R. and P. W. Moore (2008). "Reversible mechanical actuation of elastomeric nanopores." Nanotechnology 19(47).

87) N.P. Malvern._nod. < http://www.malvern.com/>. 28 October 2010.

88) N.P. IZON._n.d. http://www.izon.com/. 15 November 2010. <

89) Warr, R., Broom, M. et al. Personal communications. March 2009-November 2010.

90) Cummings, K., Laws, P., Redish, E., Cooney, P. Understanding Physics. United States of America: John Wiley \& Sons, Inc., 2004.

91) N.P. Sigma Aldrich._n.d. < http://www.sigmaaldrich.com/safety-center.html>. 20 January 2010

92) N.P. BBInternational._n.d. < http://www.bbigold.com/>. 10 March 2010.

93) Deng, T.-S., Q.-F. Zhang, et al. (2009). "One-step synthesis of highly monodisperse hybrid silica spheres in aqueous solution." Journal of Colloid and Interface Science 329(2): 292-299.

94) Pashley, R. M. and M. E. Karaman (2005). Introduction, John Wiley \& Sons, Ltd.

95) Pashley, R. M. and M. E. Karaman (2005). Charged Colloids, John Wiley \& Sons, Ltd.

96) Njoki, P. N., J. Luo, et al. "Aggregative Growth in the Size-Controlled Growth of Monodispersed Gold Nanoparticles." Langmuir 26(16): 13622-13629.

97) Roberts, G. S., D. Kozak, et al. "Tunable Nano/Micropores for Particle Detection and Discrimination: Scanning Ion Occlusion Spectroscopy." Small 6(23): 26532658. 
98) DeBlois, R. W., C. P. Bean, et al. (1977). "Electrokinetic measurements with submicron particles and pores by the resistive pulse technique." Journal of Colloid and Interface Science 61(2): 323-335.

99) Glossop, B. Email. April 2011.

100) Tilley, R; Siah, W.R. Personal Communication, November 2009. 